\title{
Complex networks: Structure and dynamics
}

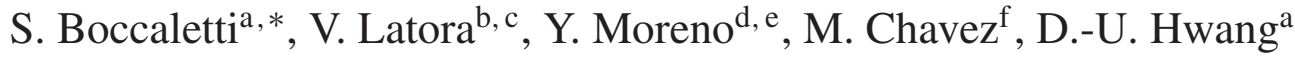 \\ ${ }^{a}$ CNR-Istituto dei Sistemi Complessi, Largo E. Fermi, 6, 50125 Florence, Italy \\ ${ }^{\mathrm{b}}$ Dipartimento di Fisica e Astronomia, Universitá di Catania, Via S. Sofia, 64, 95123 Catania, Italy \\ ${ }^{\mathrm{c}}$ Istituto Nazionale di Fisica Nucleare, Sezione di Catania, Via S. Sofia, 64, 95123 Catania, Italy \\ ${ }^{\mathrm{d}}$ Instituto de Biocomputación y Física de Sistemas Complejos, Universidad de Zaragoza, Zaragoza 50009, Spain \\ ${ }^{\mathrm{e}}$ Departamento de Fisica Teórica, Universidad de Zaragoza, Zaragoza 50009, Spain \\ ${ }^{\mathrm{f}}$ Laboratoire de Neurosciences Cognitives et Imagerie Cérébrale (LENA) CNRS UPR-640, Hôpital de la Salpêtrière. 47 Bd. de l'Hôpital, \\ 75651 Paris CEDEX 13, France
}

Accepted 27 October 2005

Available online 10 January 2006

editor: I. Procaccia

\begin{abstract}
Coupled biological and chemical systems, neural networks, social interacting species, the Internet and the World Wide Web, are only a few examples of systems composed by a large number of highly interconnected dynamical units. The first approach to capture the global properties of such systems is to model them as graphs whose nodes represent the dynamical units, and whose links stand for the interactions between them. On the one hand, scientists have to cope with structural issues, such as characterizing the topology of a complex wiring architecture, revealing the unifying principles that are at the basis of real networks, and developing models to mimic the growth of a network and reproduce its structural properties. On the other hand, many relevant questions arise when studying complex networks' dynamics, such as learning how a large ensemble of dynamical systems that interact through a complex wiring topology can behave collectively. We review the major concepts and results recently achieved in the study of the structure and dynamics of complex networks, and summarize the relevant applications of these ideas in many different disciplines, ranging from nonlinear science to biology, from statistical mechanics to medicine and engineering.
\end{abstract}

(C) 2005 Elsevier B.V. All rights reserved.

PACS: 05.45. - a

\section{Contents}

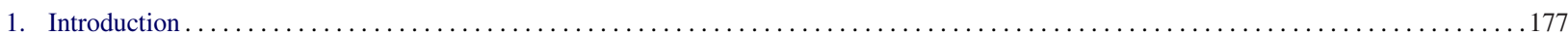

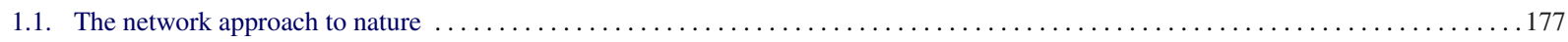

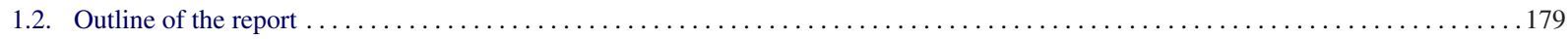

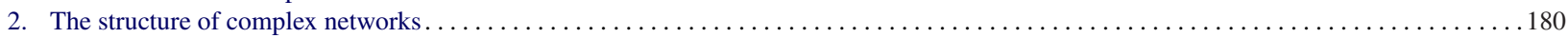

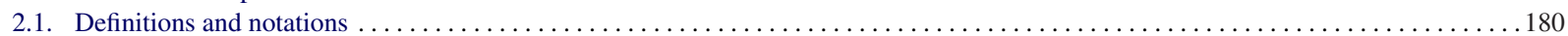

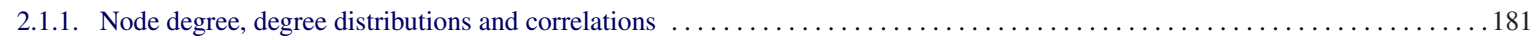

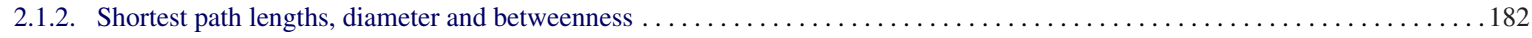

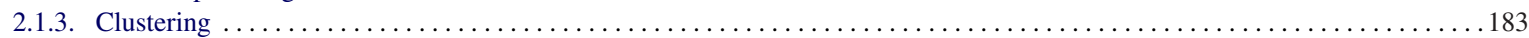

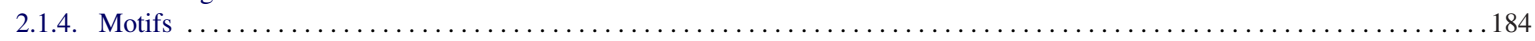

\footnotetext{
* Corresponding author.

E-mail address: stefano@ino.it (S. Boccaletti).
} 


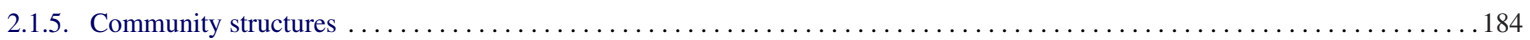

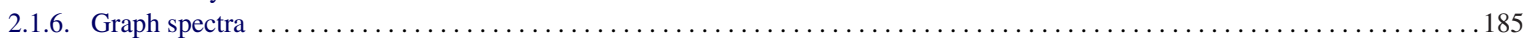

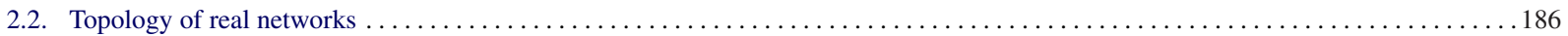

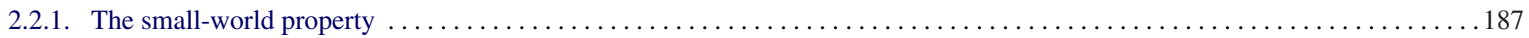

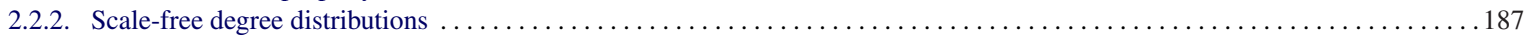

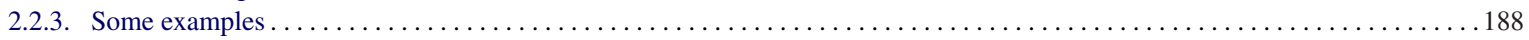

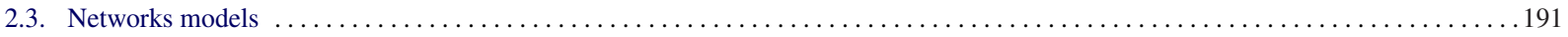

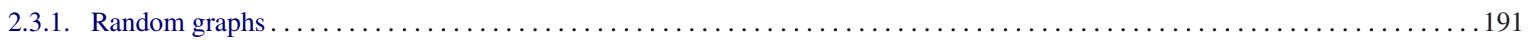

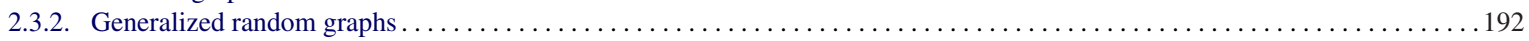

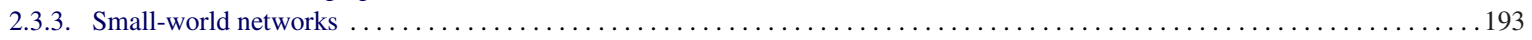

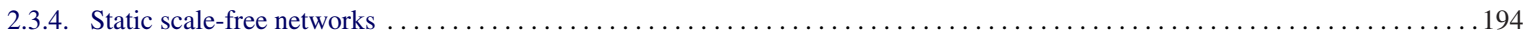

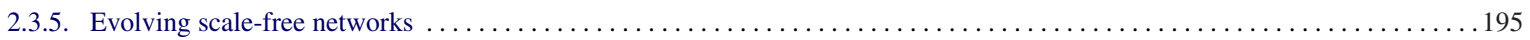

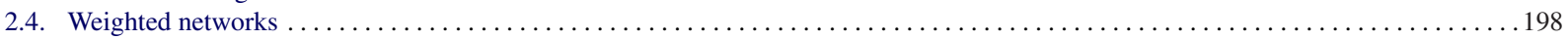

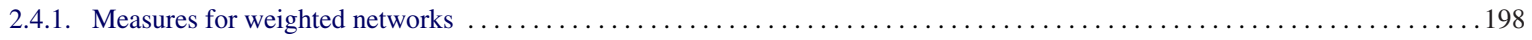

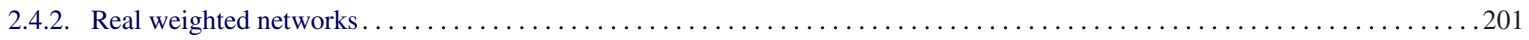

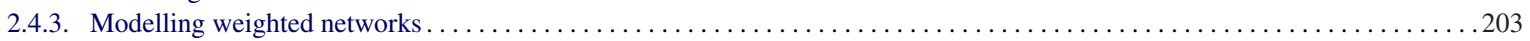

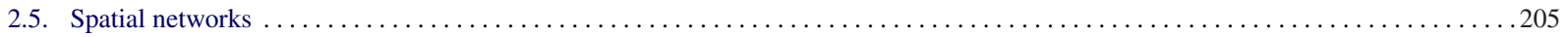

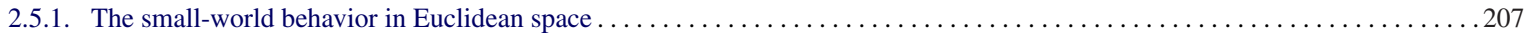

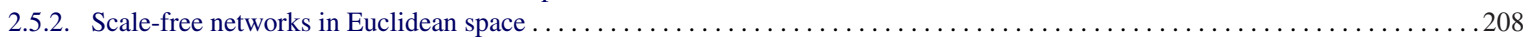

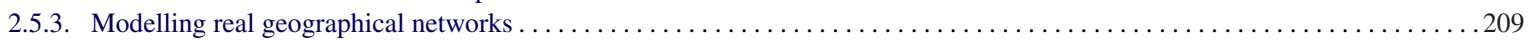

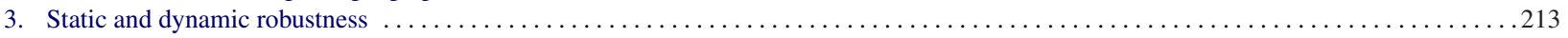

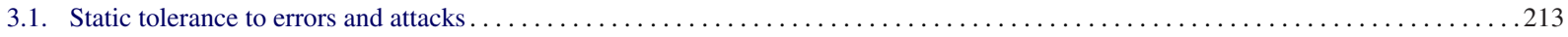

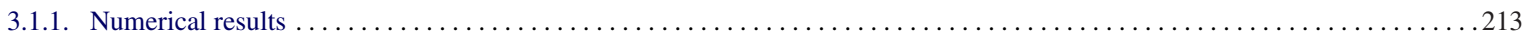

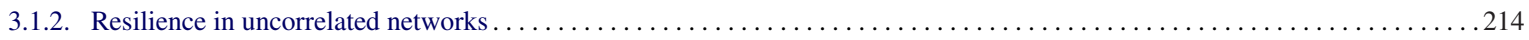

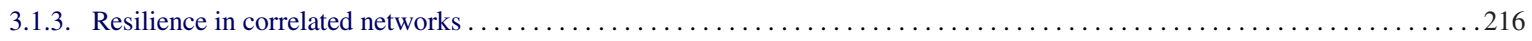

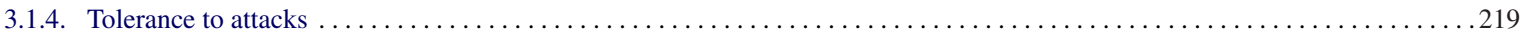

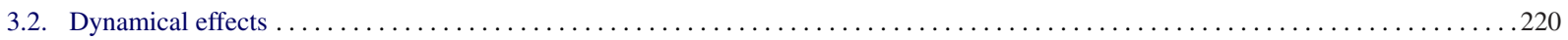

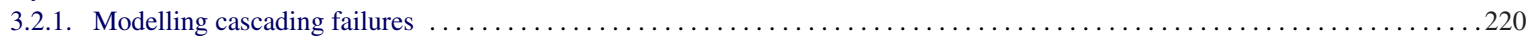

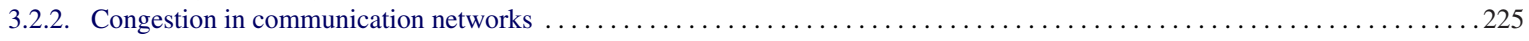

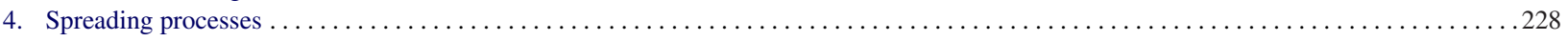

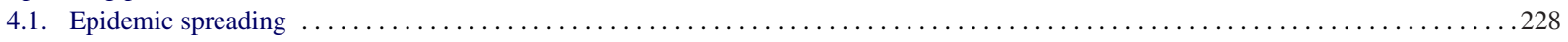

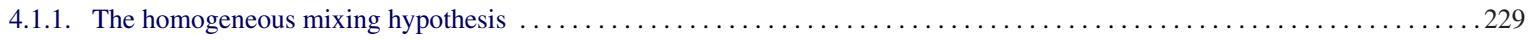

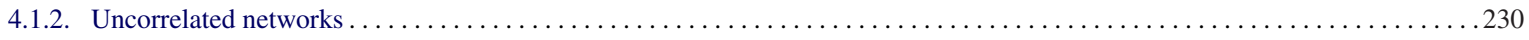

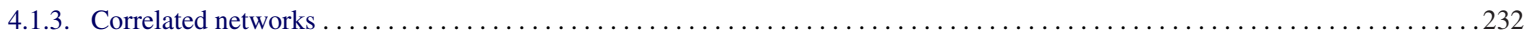

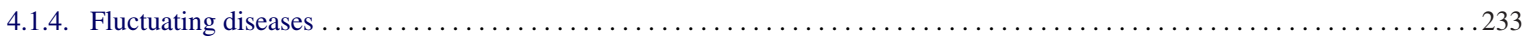

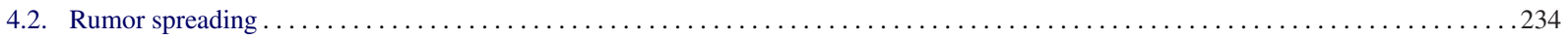

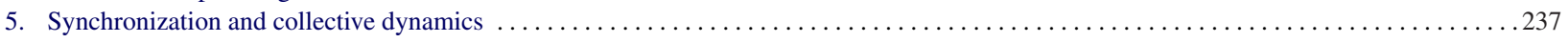

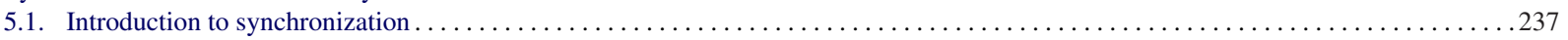

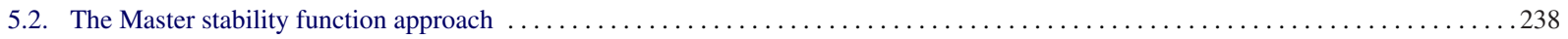

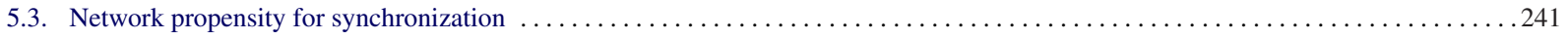

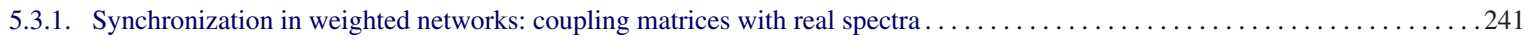

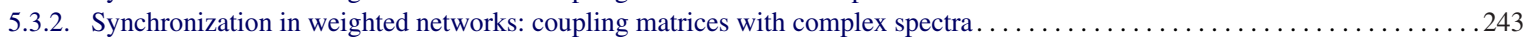

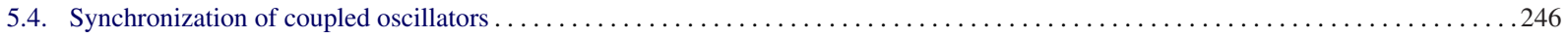

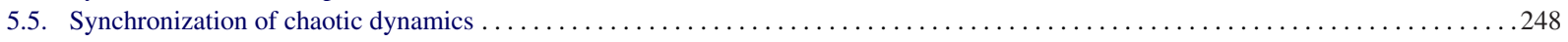

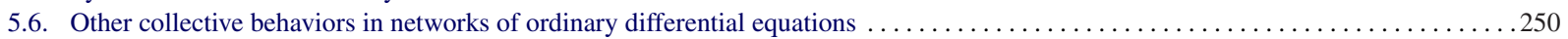

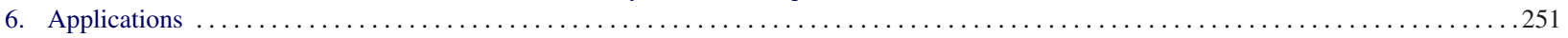

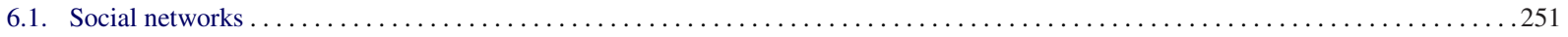

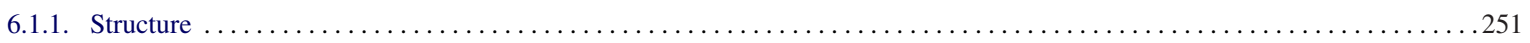

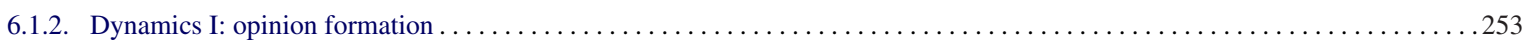

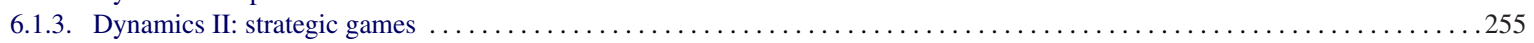

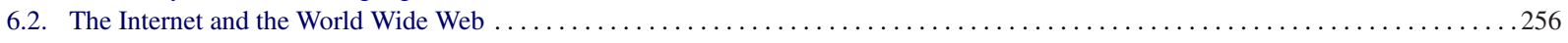

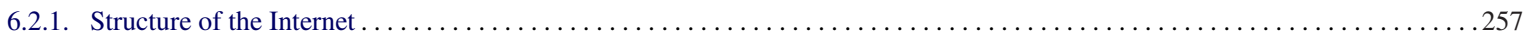

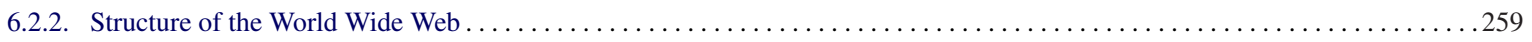

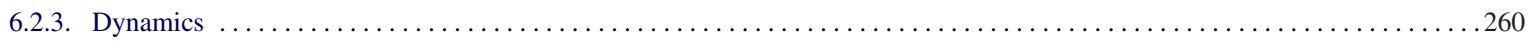

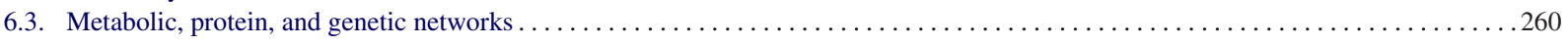

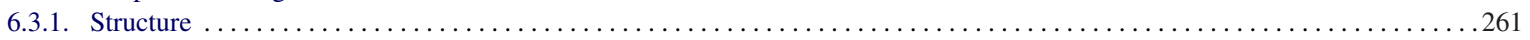

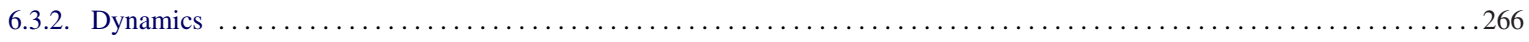

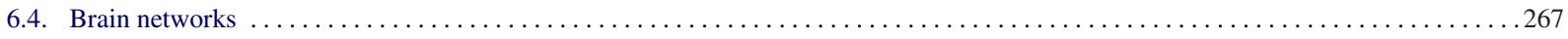

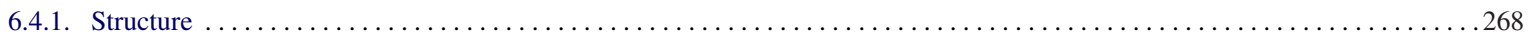

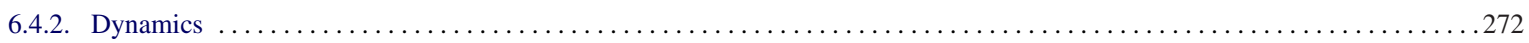

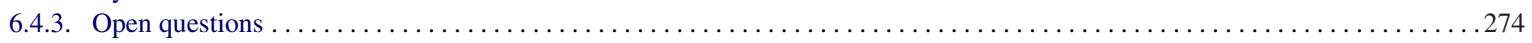

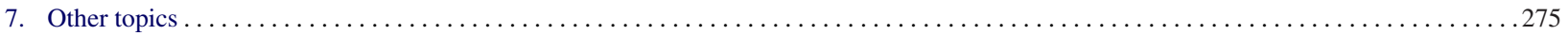




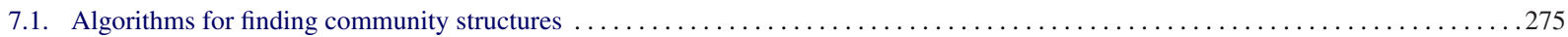

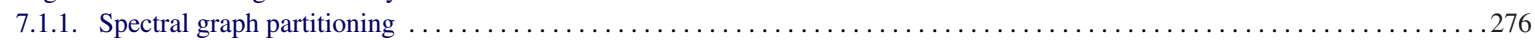

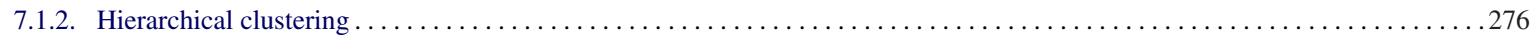

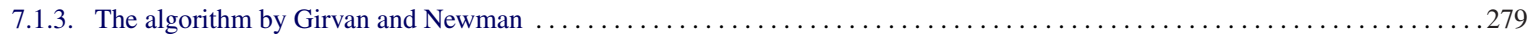

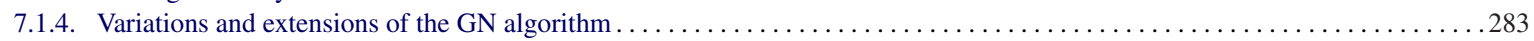

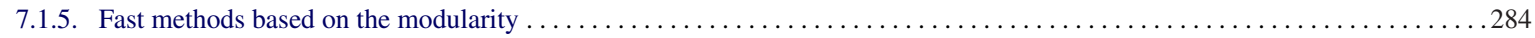

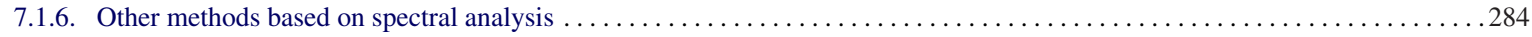

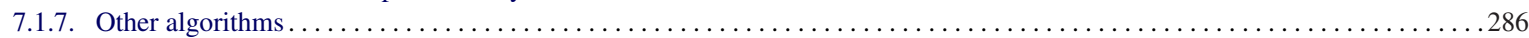

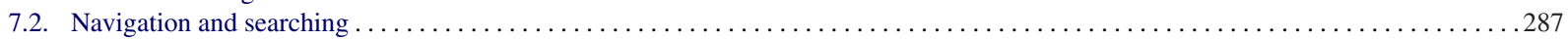

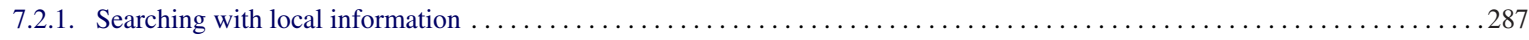

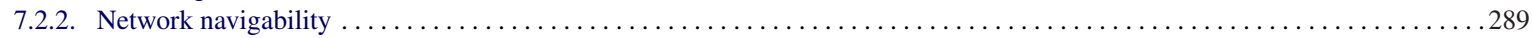

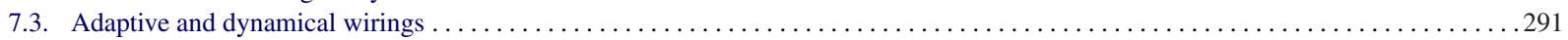

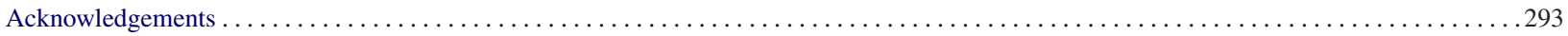

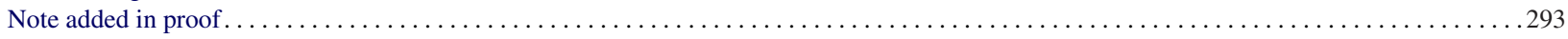

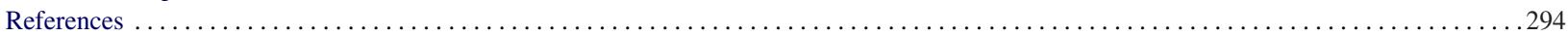

\section{Introduction}

\subsection{The network approach to nature}

Networks are all around us, and we are ourselves, as individuals, the units of a network of social relationships of different kinds and, as biological systems, the delicate result of a network of biochemical reactions. Networks can be tangible objects in the Euclidean space, such as electric power grids, the Internet, highways or subway systems, and neural networks. Or they can be entities defined in an abstract space, such as networks of acquaintances or collaborations between individuals.

Historically, the study of networks has been mainly the domain of a branch of discrete mathematics known as graph theory. Since its birth in 1736, when the Swiss mathematician Leonhard Euler published the solution to the Königsberg bridge problem (consisting in finding a round trip that traversed each of the bridges of the prussian city of Königsberg exactly once), graph theory has witnessed many exciting developments and has provided answers to a series of practical questions such as: what is the maximum flow per unit time from source to sink in a network of pipes, how to color the regions of a map using the minimum number of colors so that neighboring regions receive different colors, or how to fill $n$ jobs by $n$ people with maximum total utility. In addition to the developments in mathematical graph theory, the study of networks has seen important achievements in some specialized contexts, as for instance in the social sciences. Social networks analysis started to develop in the early 1920s and focuses on relationships among social entities, as communication between members of a group, trades among nations, or economic transactions between corporations.

The last decade has witnessed the birth of a new movement of interest and research in the study of complex networks, i.e. networks whose structure is irregular, complex and dynamically evolving in time, with the main focus moving from the analysis of small networks to that of systems with thousands or millions of nodes, and with a renewed attention to the properties of networks of dynamical units. This flurry of activity, triggered by two seminal papers, that by Watts and Strogatz on small-world networks, appeared in Nature in 1998, and that by Barabási and Albert on scale-free networks appeared one year later in Science, has seen the physics' community among the principal actors, and has been certainly induced by the increased computing powers and by the possibility to study the properties of a plenty of large databases of real networks. These include transportation networks, phone call networks, the Internet and the World Wide Web, the actors' collaboration network in movie databases, scientific coauthorship and citation networks from the Science Citation Index, but also systems of interest in biology and medicine, as neural networks or genetic, metabolic and protein networks.

The massive and comparative analysis of networks from different fields has produced a series of unexpected and dramatic results. The first issue that has been faced is certainly structural. The research on complex networks begun with the effort of defining new concepts and measures to characterize the topology of real networks. The main result has been the identification of a series of unifying principles and statistical properties common to most of the real networks considered. A relevant property regards the degree of a node, that is the number of its direct connections to other nodes. In real networks, the degree distribution $P(k)$, defined as the probability that a node chosen uniformly at random has degree $k$ or, equivalently, as the fraction of nodes in the graph having degree $k$, significantly deviates from the Poisson distribution expected for a random graph and, in many cases, exhibits a power law (scale-free) tail with an exponent 
$\gamma$ taking a value between 2 and 3. Moreover, real networks are characterized by correlations in the node degrees, by having relatively short paths between any two nodes (small-world property), and by the presence of a large number of short cycles or specific motifs.

These empirical findings have initiated a revival of network modelling, since the models proposed in mathematical graph theory turned out to be very far from the real needs. Scientists had to do with the development of new models to mimic the growth of a network and to reproduce the structural properties observed in real topologies. The structure of a real network is the result of the continuous evolution of the forces that formed it, and certainly affects the function of the system. So that this stage of the research was motivated by the expectancy that understanding and modelling the structure of a complex network would lead to a better knowledge of its evolutionary mechanisms, and to a better cottoning on its dynamical and functional behavior.

And, indeed, it was shown that the coupling architecture has important consequences on the network functional robustness and response to external perturbations, as random failures, or targeted attacks. At the same time, it outcropped for the first time the possibility of studying the dynamical behavior of large assemblies of dynamical systems interacting via complex topologies, as the ones observed empirically. This led to a series of evidences pointing to the crucial role played by the network topology in determining the emergence of collective dynamical behavior, such as synchronization, or in governing the main features of relevant processes that take place in complex networks, such as the spreading of epidemics, information and rumors.

A number of review articles [1-4] and books [5-8] on complex networks, which the reader may find useful to consult, have already appeared in the literature. Watts' pioneering book on the subject deals with the structure and the dynamics of small-world networks [5], while Strogatz' review article in the Nature's special issue on complex systems contains a discussion on networks of dynamical units [1]. Albert and Barabási [2], and Dorogovtsev and Mendes [3,7] have mainly focused their reviews on models of growing graphs, from the point of view of statistical mechanics. The review by Newman is a critical account on the field [4], containing an accurate list of references, an exhaustive overview on structural properties, measures and models, and also a final chapter devoted to processes taking place on networks. Four other references are worthwhile to mention at the beginning of this Report. They are the collection of contributed papers edited by Bornholdt and Schuster [6], that edited by Pastor-Satorras et al. [9], that edited by Ben-Naim et al. [10], and the book by Pastor-Satorras and Vespignani on the analysis and modelling of the Internet [8]. There is also a series of popular books on complex networks available on the market for the lay audience [11-13]. See for instance Buchanan's Nexus, for having the point of view of a science journalist on the field [11]. Furthermore, a variety of related books dealing with networks in specific fields of research has been published. In the context of graph theory, the books by Bollobás [14,15], West [16] and Harary [17] deserve to be quoted. The textbooks by Wasserman and Faust [18] and by Scott [19] are widely known among people working in social networks analysis. Refs. [20-22] are, instead, useful sources for the description of the standard graph algorithms.

Is this subject deserving another report? At least three reasons have motivated our work.

The first is that new research lines have emerged, covering novel topics and problems in network structure. An example is the fresh and increasingly challenging care to study weighted networks, i.e. networks in which a real number is associated to each link. This is motivated by the fact that in most of the real cases a complex topology is often associated with a large heterogeneity in the capacity and intensity of the connections. Paradigmatic cases are the existence of strong and weak ties between individuals in social systems, different capabilities of transmitting electric signals in neural networks, unequal traffic on the Internet. Ignoring such a diversity in the interactions would mean leaving away a lot of information on complex networks which is, instead, available and very useful for their characterization. A further novel topic concerns spatial networks. While most of the early works on complex networks have focused on the characterization of the topological properties, the spatial aspect has received less attention, when not neglected at all. However, it is not surprising that the topology could be constrained by the geographical embedding. For instance, the long range connections in a spatial network are constrained by the Euclidean distance, this having important consequences on the network's statistical properties. Also the degree is constrained because the number of edges that can be connected to a single node is limited by the physical space to connect them. This is particularly evident in planar networks (e.g. networks forming vertices whenever two edges cross), as urban streets, where only a small number of streets can cross in an intersection. And even in non-planar spatial networks, such as airline networks the number of connections is limited by the space available at the airport. These facts contribute to make spatial networks different from other complex networks. Along with a full account on structural properties, the present review includes all this novel material, and its many applications to relevant concrete situations. 
The second reason is that most of the interest in the subject has lately switched to investigate the dynamical behavior of networks, with a special emphasis on how the network structure affects the properties of a networked dynamical system. An example is the concerned attention to study the emergence of collective synchronized dynamics in complex networks, from the point of view of relating the propensity for synchronization of a network to the interplay between topology and local properties of the coupled dynamical systems. This phenomenon, indeed, represents a crucial feature in many relevant circumstances. For instance, evidence exists that some brain diseases are the result of an abnormal and, some times, abrupt synchronization of a large number of neural populations, so that the investigation on the network mechanisms involved in the generation, maintenance and propagation of the epileptic disorders is an issue nowadays at the forefront of neuroscience. Synchronization phenomena are very relevant also in sociology to gather a better understanding of the mechanisms underlying the formation of social collective behaviors, as the sudden emergence of new habits, fashions or leading opinions. A large portion of the second part of this Report is devoted to summarize the main achievements that have been obtained so far in dealing with collective behaviors in complex networks, reviewing the major ideas and concepts that have been developed, and assessing the rigorous results that are nowadays available.

Finally, we present a survey of a series of topics that are currently attracting much attention in the scientific community. These include the problem of building manageable algorithms to find community structures, the issue of searching within a complex network, and the modelling of adaptive networks.

Community structures are an important property of complex networks. For example, tightly connected groups of nodes in a social network represent individuals belonging to social communities, tightly connected groups of nodes in the World Wide Web often correspond to pages on common topics, while communities in cellular and genetic networks are somehow related to functional modules. Consequently, finding the communities within a network is a powerful tool for understanding the functioning of the network, as well as for identifying a hierarchy of connections within a complex architecture.

Another relevant problem is how to reach a node of the network from another one, by navigating the network often in the absence of information on the global structure, or how to optimize a searching procedure based only on some local information on the network topology.

Adaptive and dynamical wirings are a peculiarity of those networks that are themselves dynamical entities. This means that the topology is not fixed, or grown, once forever. Instead it is allowed to evolve and adapt in time, driven by some external action, or by the action of the internal elements, or following specific predetermined evolving rules. This step forward has been motivated by the need of suitably modelling some specific cases, such as genetic regulatory networks, ecosystems, financial markets, as well as to properly describe a series of technologically relevant problems emerging, e.g., in mobile and wireless connected units. In some cases, the research work has just begun, and, even though the results are not so firmly established, we believe that the state of the art calls for future relevant achievements.

\subsection{Outline of the report}

The Report is organized as follows.

Chapter 2 is about network structure. We describe some of the common properties observed in the topology of real networks, and how they are measured. We then briefly review the main models that have been proposed over the years, focusing on random graphs, small-world models and scale-free networks. Finally, we give a special emphasis to the study and modelling of weighted networks, as well as networks with a spatial structure.

In Chapter 3 we discuss the network robustness against external perturbations consisting in the malfunctioning, or the deliberate damage, of some of its components. We review both static and dynamical approaches. Specifically, we describe percolation processes on uncorrelated and correlated networks, cascading failures, and congestion in transportation and communication networks.

In Chapter 4 we consider cellular automata on complex topologies and we analyze a series of models for the spreading of epidemics and rumors.

Chapter 5 is concerned with the emergence of collective synchronized dynamics in complex networks. In this context, we review the most significant advancement represented by the Master Stability Function approach, giving conditions in the wiring topology that maximize the propensity for synchronization of a network. We furthermore consider networks whose dynamical units evolve nonlinearly, and we review the main results obtained with networks of chaotic maps, networks of chaotic systems, and with networks of periodic oscillators. 
Chapter 6 summarizes some applications to real networks such as the Internet and the World Wide Web, social networks, networks describing the interaction between cell components, and neural networks. The chapter contains issues concerning both the structure and the dynamics of such networks.

Finally, in Chapter 7 we consider three topics that have recently attracted a large interest in the scientific community. We first discuss algorithms for partitioning large networks into community structures. We then review the recent advancements in finding reliable and fast ways for navigation and searching in a complex network. The chapter ends with a discussion of adaptive and dynamical wirings.

\section{The structure of complex networks}

The material in this chapter is intended to serve as a brief account of the recent developments in the characterization and modelling of the structural properties of a network. We shall first introduce definitions and notations, and discuss the basic quantities used to describe the topology of a network. Then, we shall move to the analysis of the properties observed in real networks, and provide the reader with a brief review of the models motivated by the empirical observations. The chapter ends with a survey on the recent achievements in the study of weighted networks and spatial networks.

\subsection{Definitions and notations}

Graph theory [14-17] is the natural framework for the exact mathematical treatment of complex networks and, formally, a complex network can be represented as a graph. A undirected (directed) graph $G=(\mathscr{N}, \mathscr{L}$ ) consists of two sets $\mathscr{N}$ and $\mathscr{L}$, such that $\mathscr{N} \neq \emptyset$ and $\mathscr{L}$ is a set of unordered (ordered) pairs of elements of $\mathscr{N}$. The elements of $\mathscr{N} \equiv\left\{n_{1}, n_{2}, \ldots, n_{N}\right\}$ are the nodes (or vertices, or points) of the graph $G$, while the elements of $\mathscr{L} \equiv\left\{l_{1}, l_{2}, \ldots, l_{K}\right\}$ are its links (or edges, or lines). The number of elements in $\mathscr{N}$ and $\mathscr{L}$ are denoted by $N$ and $K$, respectively. In the following of the report we will indicate a graph as $G(N, K)=(\mathscr{N}, \mathscr{L})$, or simply $G(N, K)$ or $G_{N, K}$, whenever it is necessary to emphasize the number of nodes (the size of the graph) and links in the graph.

A node is usually referred to by its order $i$ in the set $\mathscr{N}$. In a undirected graph, each of the links is defined by a couple of nodes $i$ and $j$, and is denoted as $(i, j)$ or $l_{i j}$. The link is said to be incident in nodes $i$ and $j$, or to join the two nodes; the two nodes $i$ and $j$ are referred to as the end-nodes of link $(i, j)$. Two nodes joined by a link are referred to as adjacent or neighboring. In a directed graph, the order of the two nodes is important: $l_{i j}$ stands for a link from $i$ to $j$, and $l_{i j} \neq l_{j i}$. The usual way to picture a graph is by drawing a dot for each node and joining two dots by a line if the two corresponding nodes are connected by a link. How these dots and lines are drawn is irrelevant, and the only thing that matters is which pairs of nodes form a link and which ones do not. Examples of a undirected graph and of a directed graph, both with $N=7$ and $K=14$, are shown in Fig. 2.1(a) and (b), respectively. Note that the picture does not contain loops, i.e. links from a node to itself, nor multiple edges, i.e. couples of nodes connected by more than one

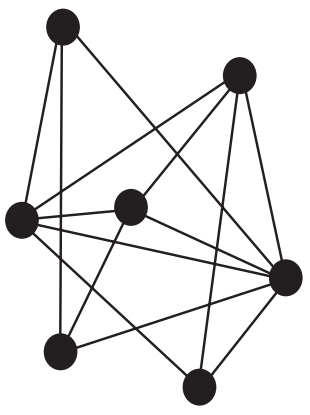

(a)

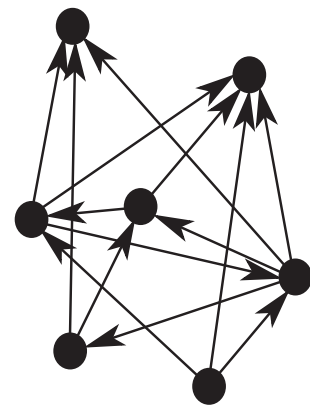

(b)

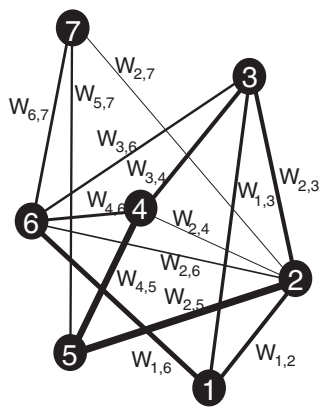

(c)

Fig. 2.1. Graphical representation of a undirected (a), a directed (b), and a weighted undirected (c) graph with $N=7$ nodes and $K=14$ links. In the directed graph, adjacent nodes are connected by arrows, indicating the direction of each link. In the weighted graph, the values $w_{i, j}$ reported on each link indicate the weights of the links, and are graphically represented by the link thicknesses. 
link, since these elements are not allowed by the standard definition of graph given above. Graphs with either of these elements are called multigraphs $[15,18]$. In this report, the emphasis will be on graphs rather than multigraphs and, unless explicitly mentioned, the term graph will be used to refer to a undirected graph. Weighted graphs will receive a special attention and will be discussed in Section 2.4.

For a graph $G$ of size $N$, the number of edges $K$ is at least 0 and at most $N(N-1) / 2$ (when all the nodes are pairwise adjacent). $G$ is said to be sparse if $K \ll N^{2}$ and dense if $K=\mathcal{O}\left(N^{2}\right)$. A graph $G_{N, K}$ is said a complete $\mathrm{N}$-graph if $K=\left(\begin{array}{c}N \\ 2\end{array}\right)=N(N-1) / 2$, and is denoted by $K_{N} . K_{3}$ is called a triangle.

A subgraph $G^{\prime}=\left(\mathscr{N}^{\prime}, \mathscr{L}^{\prime}\right)$ of $G=(\mathscr{N}, \mathscr{L})$ is a graph such that $\mathscr{N}^{\prime} \subseteq \mathscr{N}$ and $\mathscr{L}^{\prime} \subseteq \mathscr{L}$. If $G^{\prime}$ contains all links of $G$ that join two nodes in $\mathscr{N}^{\prime}$, then $G^{\prime}$ is said to be the subgraph induced by $\mathscr{N}^{\prime}$ and is denoted as $G^{\prime}=G\left[\mathscr{N}^{\prime}\right]$. A subgraph is said to be maximal with respect to a given property if it cannot be extended without loosing that property. Of particular relevance for some of the definitions given in the following subsections is the subgraph of the neighbors of a given node $i$, denoted as $G_{i} . G_{i}$ is defined as the subgraph induced by $\mathscr{N}_{i}$, the set of nodes adjacent to $i$, i.e. $G_{i}=G\left[\mathscr{N}_{i}\right]$.

A central concept in graph theory is that of reachability of two different nodes of a graph. In fact, two nodes that are not adjacent may nevertheless be reachable from one to the other. A walk from node $i$ to node $j$ is an alternating sequence of nodes and edges (a sequence of adjacent nodes) that begins with $i$ and ends with $j$. The length of the walk is defined as the number of edges in the sequence. A trail is a walk in which no edge is repeated. A path is a walk in which no node is visited more than once. The walk of minimal length between two nodes is known as shortest path or geodesic. A cycle is a closed walk, of at least three nodes, in which no edge is repeated. A cycle of length $k$ is usually said a $k$-cycle and denoted as $C_{k}$. $C_{3}$ is a triangle $\left(C_{3}=K_{3}\right), C_{4}$ is called a quadrilater, $C_{5}$ a pentagon, and so on. A graph is said to be connected if, for every pair of distinct nodes $i$ and $j$, there is a path from $i$ to $j$, otherwise it is said unconnected or disconnected. A component of the graph is a maximally connected induced subgraph. A giant component is a component whose size is of the same order as $N$.

It is often useful to consider a matricial representation of a graph. A graph $G=(\mathscr{N}, \mathscr{L})$ can be completely described by giving the adjacency (or connectivity) matrix $\mathscr{A}$, a $N \times N$ square matrix whose entry $a_{i j}(i, j=1, \ldots, N)$ is equal to 1 when the link $l_{i j}$ exists, and zero otherwise. The diagonal of the adjacency matrix contains zeros. This is thus a symmetric matrix for undirected graphs. An alternative possibility is to use the incidence matrix $\mathscr{B}$, a $N \times K$ matrix whose entry $b_{i k}$ is equal to 1 whenever the node $i$ is incident with the link $l_{k}$, and zero otherwise.

\subsubsection{Node degree, degree distributions and correlations}

The degree (or connectivity) $k_{i}$ of a node $i$ is the number of edges incident with the node, and is defined in terms of the adjacency matrix $\mathscr{A}$ as:

$$
k_{i}=\sum_{j \in \mathscr{N}} a_{i j}
$$

If the graph is directed, the degree of the node has two components: the number of outgoing links $k_{i}^{\text {out }}=\sum_{j} a_{i j}$ (referred to as the out-degree of the node), and the number of ingoing links $k_{i}^{\text {in }}=\sum_{j} a_{j i}$ (referred to as the in-degree of the node). The total degree is then defined as $k_{i}=k_{i}^{\text {out }}+k_{i}^{\text {in }}$. A list of the node degrees of a graph is called the degree sequence.

The most basic topological characterization of a graph $G$ can be obtained in terms of the degree distribution $P(k)$, defined as the probability that a node chosen uniformly at random has degree $k$ or, equivalently, as the fraction of nodes in the graph having degree $k$. Alternatively, the degree distribution is denoted as $P_{k}$, or $p_{k}$, to indicate that the variable $k$ assumes non-negative integer values.

In the case of directed networks one needs to consider two distributions, $P\left(k^{\text {in }}\right)$ and $P\left(k^{\text {out }}\right)$. Information on how the degree is distributed among the nodes of a undirected network can be obtained either by a plot of $P(k)$, or by the calculation of the moments of the distribution. The $n$-moment of $P(k)$ is defined as:

$$
\left\langle k^{n}\right\rangle=\sum_{k} k^{n} P(k) .
$$

The first moment $\langle k\rangle$ is the mean degree of $G$. The second moment measures the fluctuations of the connectivity distribution, and, as we shall see in Chapters 3 and 4, the divergence of $\left\langle k^{2}\right\rangle$ in the limit of infinite graph size, radically changes the behavior of dynamical processes that take place over the graph. 
The degree distribution completely determines the statistical properties of uncorrelated networks. However, as we shall see, a large number of real networks are correlated in the sense that the probability that a node of degree $k$ is connected to another node of degree, say $k^{\prime}$, depends on $k$. In these cases, it is necessary to introduce the conditional probability $P\left(k^{\prime} \mid k\right)$, being defined as the probability that a link from a node of degree $k$ points to a node of degree $k^{\prime} . P\left(k^{\prime} \mid k\right)$ satisfies the normalization $\sum_{k^{\prime}} P\left(k^{\prime} \mid k\right)=1$, and the degree detailed balance condition $k P\left(k^{\prime} \mid k\right) P(k)=$ $k^{\prime} P\left(k \mid k^{\prime}\right) P\left(k^{\prime}\right)[23,24]$. For uncorrelated graphs, in which $P\left(k^{\prime} \mid k\right)$ does not depend on $k$, the detailed balance condition and the normalization give $P\left(k^{\prime} \mid k\right)=k^{\prime} P\left(k^{\prime}\right) /\langle k\rangle$.

Although the degree correlations are formally characterized by $P\left(k^{\prime} \mid k\right)$, the direct evaluation of the conditional probability gives extremely noisy results for most of the real networks because of their finite size $N$. This problem can be overcome by defining the average nearest neighbors degree of a node $i$ as

$$
k_{n n, i}=\frac{1}{k_{i}} \sum_{j \in \mathscr{N}_{i}} k_{j}=\frac{1}{k_{i}} \sum_{j=1}^{N} a_{i j} k_{j},
$$

where the sum runs on the nodes belonging to $\mathscr{N}_{i}$, the set of first neighbors of $i$. By using the definition (2.3), one can calculate the average degree of the nearest neighbors of nodes with degree $k$, denoted as $k_{n n}(k)$, obtaining an expression that implicitly incorporates the dependence on $k$ [25]. Such a quantity can be, indeed, expressed in terms of the conditional probability as

$$
k_{n n}(k)=\sum_{k^{\prime}} k^{\prime} P\left(k^{\prime} \mid k\right) .
$$

If there are no degree correlations, Eq. (2.4) gives $k_{n n}(k)=\left\langle k^{2}\right\rangle /\langle k\rangle$, i.e. $k_{n n}(k)$ is independent of $k$. Correlated graphs are classified as assortative if $k_{n n}(k)$ is an increasing function of $k$, whereas they are referred to as disassortative when $k_{n n}(k)$ is a decreasing function of $k$ [26]. In other words, in assortative networks the nodes tend to connect to their connectivity peers, while in disassortative networks nodes with low degree are more likely connected with highly connected ones. Degree correlation are usually quantified by reporting the numerical value of the slope of $k_{n n}(k)$ as a function of $k$, denoted in the following as $v$, or by calculating the Pearson correlation coefficient of the degrees at either ends of a link $[26,27]$. In Section 2.2 we shall discuss some examples of real networks showing both types of correlations.

\subsubsection{Shortest path lengths, diameter and betweenness}

Shortest paths play an important role in the transport and communication within a network. Suppose one needs to send a data packet from one computer to another through the Internet: the geodesic provides an optimal path way, since one would achieve a fast transfer and save system resources [8]. For such a reason, shortest paths have also played an important role in the characterization of the internal structure of a graph [18,19]. It is useful to represent all the shortest path lengths of a graph $G$ as a matrix $\mathscr{D}$ in which the entry $d_{i j}$ is the length of the geodesic from node $i$ to node $j$. The maximum value of $d_{i j}$ is called the diameter of the graph, and will be indicated in the following as $\operatorname{Diam}(G)$. A measure of the typical separation between two nodes in the graph is given by the average shortest path length, also known as characteristic path length, defined as the mean of geodesic lengths over all couples of nodes [5,28]:

$$
L=\frac{1}{N(N-1)} \sum_{i, j \in \mathscr{N}, i \neq j} d_{i j} .
$$

A problem with this definition is that $L$ diverges if there are disconnected components in the graph. One possibility to avoid the divergence is to limit the summation in formula (2.5) only to couples of nodes belonging to the largest connected component [28]. An alternative approach, that is useful in many cases (see, for instance, Section 3.1.1), is to consider the harmonic mean [29] of geodesic lengths, and to define the so-called efficiency of $G$ as [30,31]:

$$
E=\frac{1}{N(N-1)} \sum_{i, j \in \mathscr{N}, i \neq j} \frac{1}{d_{i j}} .
$$

Such a quantity is an indicator of the traffic capacity of a network, and avoids the divergence of formula (2.5), since any couple of nodes belonging to disconnected components of the graph yields a contribution equal to zero to the 
summation in formula (2.6). The mathematical properties of the efficiency have been investigated in Ref. [32]. See Refs. [33-35] for some recent applications of the efficiency, and Refs. [32,36] for some extensions of formula (2.6).

The communication of two non-adjacent nodes, say $j$ and $k$, depends on the nodes belonging to the paths connecting $j$ and $k$. Consequently, a measure of the relevance of a given node can be obtained by counting the number of geodesics going through it, and defining the so-called node betweenness. Together with the degree and the closeness of a node (defined as the inverse of the average distance from all other nodes), the betweenness is one of the standard measures of node centrality, originally introduced to quantify the importance of an individual in a social network [18,19,37]. More precisely, the betweenness $b_{i}$ of a node $i$, sometimes referred to also as load, is defined as [18,19,38,39]:

$$
b_{i}=\sum_{j, k \in \mathscr{N}, j \neq k} \frac{n_{j k}(i)}{n_{j k}},
$$

where $n_{j k}$ is the number of shortest paths connecting $j$ and $k$, while $n_{j k}(i)$ is the number of shortest paths connecting $j$ and $k$ and passing through $i$. See Refs. [20-22] for a description of the standard algorithms to find shortest paths (as the Dijkstra's algorithm, or the breadth-first search method discussed in Section 7.2), and Refs. [40,41] for fast algorithms recently proposed to calculate the betweenness. Betweenness distributions have been investigated in Refs. [42-47]. Betweenness-betweenness correlations and betweenness-degree correlations have been studied respectively in Ref. [42] and in Refs. [48-50].

The concept of betweenness can be extended also to the edges. The edge betweenness is defined as the number of shortest paths between pairs of nodes that run through that edge [51]. This latter quantity will be used extensively in Section 5.3, as well as in Section 7.1.3.

\subsubsection{Clustering}

Clustering, also known as transitivity, is a typical property of acquaintance networks, where two individuals with a common friend are likely to know each other [18]. In terms of a generic graph $G$, transitivity means the presence of a high number of triangles. This can be quantified by defining the transitivity $T$ of the graph as the relative number of transitive triples, i.e. the fraction of connected triples of nodes (triads) which also form triangles [4,52,53]:

$$
T=\frac{3 \times \# \text { of triangles in } G}{\# \text { of connected triples of vertices in } G} .
$$

The factor 3 in the numerator compensates for the fact that each complete triangle of three nodes contributes three connected triples, one centered on each of the three nodes, and ensures that $0 \leqslant T \leqslant 1$, with $T=1$ for $K_{N}$.

An alternative possibility is to use the graph clustering coefficient $C$, a measure introduced by Watts and Strogatz in Ref. [28], and defined as follows. A quantity $c_{i}$ (the local clustering coefficient of node $i$ ) is first introduced, expressing how likely $a_{j m}=1$ for two neighbors $j$ and $m$ of node $i$. Its value is obtained by counting the actual number of edges (denoted by $e_{i}$ ) in $G_{i}$ (the subgraph of neighbors of $i$ defined as in Section 2.1). Notice that $G_{i}$ can be, in some cases, unconnected. The local clustering coefficient is defined as the ratio between $e_{i}$ and $k_{i}\left(k_{i}-1\right) / 2$, the maximum possible number of edges in $G_{i}[5,28]$ :

$$
c_{i}=\frac{2 e_{i}}{k_{i}\left(k_{i}-1\right)}=\frac{\sum_{j, m} a_{i j} a_{j m} a_{m i}}{k_{i}\left(k_{i}-1\right)} .
$$

Fast algorithms to compute $c_{i}$ are available in Ref. [54]. The clustering coefficient of the graph is then given by the average of $c_{i}$ over all the nodes in $G$ :

$$
C=\langle c\rangle=\frac{1}{N} \sum_{i \in \mathcal{N}} c_{i} .
$$

By definition, $0 \leqslant c_{i} \leqslant 1$, and $0 \leqslant C \leqslant 1$. The differences between $C$ and $T$ are illustrated in Refs. [4,31]. It is also useful to consider $c(k)$, the clustering coefficient of a connectivity class $k$, which is defined as the average of $c_{i}$ taken over all nodes with a given degree $k$.

Various higher-order clustering coefficients have been proposed over the years, among which we recall the $k$ clustering coefficient, that accounts for $k$-neighbors [55,56], or other measures based on the internal structure of cycles 

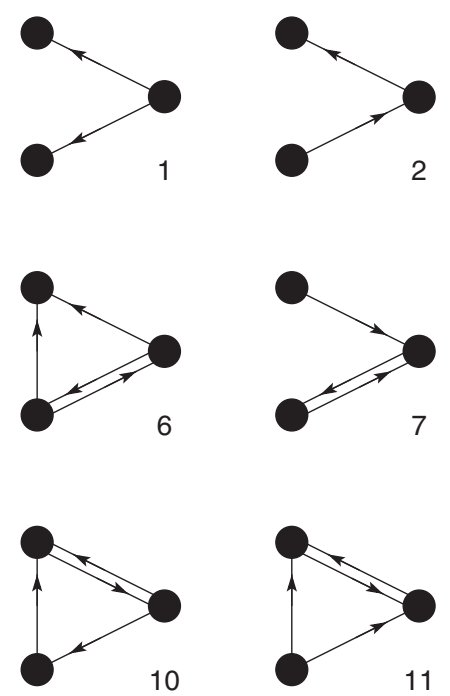

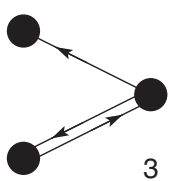

3

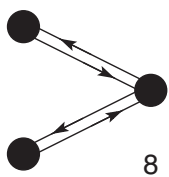

8

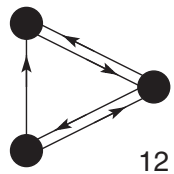

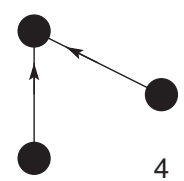

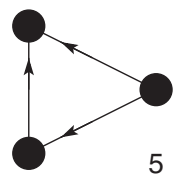

5

Fig. 2.2. All the 13 types of motifs consisting in three-nodes directed connected subgraphs.

of order four [57], or on the number of cycles of a generic order [58]. See also Refs. [59,60] for a definition of a clustering coefficient without the bias of degree correlations, Ref. [61] for a definition of triads in directed graphs and Ref. [33] for a definition of clustering in planar graphs (see Section 2.5). An alternative measure of the clustering properties of $G$ is the local efficiency, defined as [30,31]

$$
E_{\mathrm{loc}}=\frac{1}{N} \sum_{i \in \mathscr{N}} E\left(G_{i}\right),
$$

where $E\left(G_{i}\right)$ is the efficiency of $G_{i}$, evaluated by formula (2.6).

\subsubsection{Motifs}

A motif $M$ is a pattern of interconnections occurring either in a undirected or in a directed graph $G$ at a number significantly higher than in randomized versions of the graph, i.e. in graphs with the same number of nodes, links and degree distribution as the original one, but where the links are distributed at random. As a pattern of interconnections, $M$ is usually meant as a connected (undirected or directed) $n$-node graph which is a subgraph of $G$. An example of all the possible 3-node connected directed graphs is illustrated in Fig. 2.2.

The concept of motifs was originally introduced in Refs. [62-66] by Alon and coworkers, who studied small $n$ motifs in biological and other networks. The research of the significant motifs in a graph $G$ is based on matching algorithms counting the total number of occurrences of each $n$-node subgraph $M$ in the original graph and in the randomized ones. The statistical significance of $M$ is then described by the $Z$-score, defined as $[63,65]$

$$
Z_{M}=\frac{n_{M}-\left\langle n_{M}^{\mathrm{rand}}\right\rangle}{\sigma_{n_{M}}^{\mathrm{rand}}},
$$

where $n_{M}$ is the number of times the subgraph $M$ appears in $G$, and $\left\langle n_{M}^{\text {rand }}\right\rangle$ and $\sigma_{n_{M}}^{\text {rand }}$ are, respectively, the mean and standard deviation of the number of appearances in the randomized network ensemble.

\subsubsection{Community structures}

Historically, the notion of community, and the first network formalizations of the concept, have been proposed in the social sciences [18]. Given a graph $G(\mathscr{N}, \mathscr{L})$, a community (or cluster, or cohesive subgroup) is a subgraph $G^{\prime}\left(\mathscr{N}^{\prime}, \mathscr{L}^{\prime}\right)$, whose nodes are tightly connected, i.e. cohesive. Since the structural cohesion of the nodes of $G^{\prime}$ can be quantified in several different ways, there are different formal definitions of community structures. 


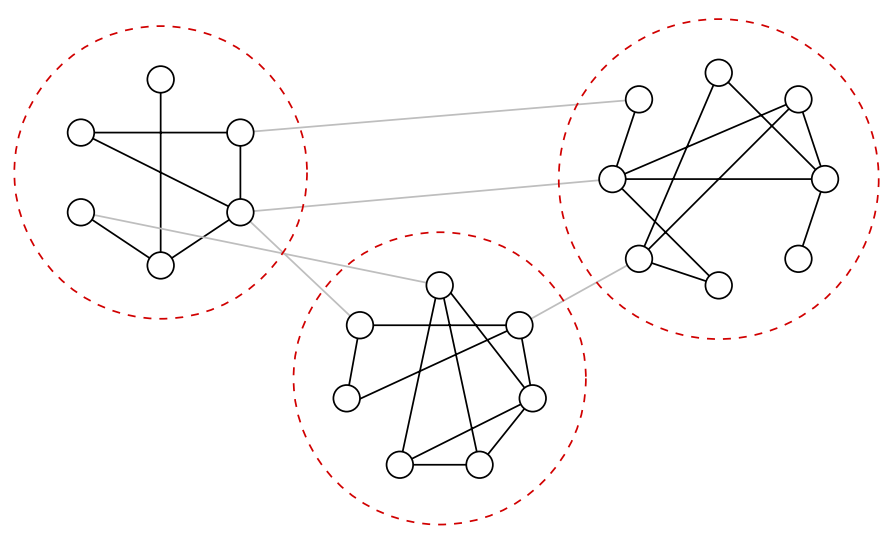

Fig. 2.3. Communities can be defined as groups of nodes such that there is a higher density of edges within groups than between them. In the case shown in figure there are three communities, denoted by the dashed circles. Reprinted figure with permission from Ref. [51]. CC 2004 by the American Physical Society.

The strongest definition requires that all pairs of community members choose each other. Such a requirement leads to the definition of a clique. A clique is a maximal complete subgraph of three or more nodes, i.e. a subset of nodes all of which are adjacent to each other, and such that no other nodes exist adjacent to all of them. This definition can be extended by weakening the requirement of adjacency into a requirement of reachability: a $n$-clique is a maximal subgraph in which the largest geodesic distance between any two nodes is no greater than $n$. When $n=1$, this definition coincides with that of a clique. 2-cliques are subgraphs in which all nodes need not to be adjacent but are reachable through at most one intermediary. In 3-cliques all nodes are reachable through at most two intermediaries, and so on. Whereas the concept of $n$-clique involves increasing the permissible path lengths, an alternative possibility to relax the strong assumption of cliques involves reducing the number of other nodes to which each node must be connected. A $k$-plex is a maximal subgraph containing $n$ nodes, in which each node is adjacent to no fewer than $n-k$ nodes in the subgraph.

A different class of definitions is based on the relative frequency of links. In this case communities are seen as groups of nodes within which connections are dense, and between which connections are sparser $[67,68]$. An example is shown in Fig. 2.3. While the simplest formal definitions in this class have been proposed in Refs. [69,70], a less stringent definition is the following: $G^{\prime}$ is a community if the sum of all degrees within $G^{\prime}$ is larger than the sum of all degrees toward the rest of the graph [71]. The definitions presented here are not the only possible choices. Several other definitions, possibly more appropriate in some cases, can be found in Ref. [18].

\subsubsection{Graph spectra}

The spectrum of a graph is the set of eigenvalues of its adjacency matrix $\mathscr{A}$ [16]. A graph $G_{N, K}$ has $N$ eigenvalues $\mu_{i}(i=1,2, \ldots, N)$, and $N$ associated eigenvectors $\mathbf{v}_{i}(i=1,2, \ldots, N)$. When $G$ is undirected, without loops or multiple edges, $\mathscr{A}$ is real and symmetric, therefore the graph has real eigenvalues $\mu_{1} \leqslant \mu_{2} \leqslant \cdots \leqslant \mu_{N}$, and the eigenvectors corresponding to distinct eigenvalues are orthogonal. When $G$ is directed, the eigenvalues can have imaginary part, as, for instance, in the tournament graph with 3 nodes. Consequently, ordering and properties of eigenvalues and eigenvectors are more complicated. This latter situation will be described in details when appropriate.

The Perron-Frobenius theorem tells that a graph (also direct) has a real eigenvalue $\mu_{N}$ associated to a real nonnegative eigenvector, and such that $|\mu| \leqslant \mu_{N}$ for each eigenvalue $\mu$. If the graph is connected, then $\mu_{N}$ has multiplicity 1 and $|\mu|<\mu_{N}$ for all eigenvalues $\mu$ different from $\mu_{N}$. The value of $\mu_{N}$ decreases when vertices or edges are removed from the graph. For a connected undirected graph, this means that the largest eigenvalue $\mu_{N}$ is not degenerate, and every component of the corresponding eigenvector $\mathbf{v}_{N}$ is non-negative. All other eigenvectors have entries with mixed signs, because are orthogonal to $\mathbf{v}_{N}$. The same theorem also states that in a connected graph either $k_{\min }<\langle k\rangle<\mu_{N}<k_{\max }$, or $k_{\min }=\langle k\rangle=\mu_{N}=k_{\max }$. 
For large graphs it is useful to define the spectral density as [72,73]

$$
\rho(\mu)=\frac{1}{N} \sum_{i=1}^{N} \delta\left(\mu-\mu_{i}\right),
$$

which approaches a continuous function as $N \rightarrow \infty$. The eigenvalues and associated eigenvectors of a graph are intimately related to important topological features such as the diameter, the number of cycles, and the connectivity properties of the graph.

For instance, the eigenvalues sum to zero because $\operatorname{Tr}(\mathscr{A})=0$, the sum of product of all pairs of eigenvalues is equal to $-K$, the sum of product of triples of eigenvalues is twice the number of triangles (3-cycles) in $G$, while the sum of higher order products is related to cycles of length $4,5, \ldots$, although they contain also contributions from shorter cycles $[16,74,75]$. Such properties are related to the fact that the $(i, j)^{\text {th }}$ entry of the matrix $\mathscr{A}^{k}$ is equal to the number of walks of length $k$ from node $i$ to node $j$ [16]. Consequently, the trace of $\mathscr{A}^{k}$ gives the sum (over nodes) of the number of walks of length $k$ from a node of the graph to itself. Since the eigenvalues of $\mathscr{A}^{k}$ are the $k$-power of the eigenvalues of $\mathscr{A}$, the trace of $\mathscr{A}^{k}$ can be easily evaluated from the eigenvalues of $\mathscr{A}$, without explicitly performing matrix products. One obtains $\operatorname{Tr}\left(\mathscr{A}^{k}\right)=\sum_{i}^{N} \mu_{i}^{k}$, that, together with the relations $\left(\sum_{i}^{N} \mu_{i}\right)^{k}=0$, gives the above mentioned properties.

This also implies that $M_{k}$, the $k$ th moment of the spectral density $\rho(\mu)$ of Eq. 2.13 (defined as $M_{k}=1 / N \sum_{i}^{N} \mu_{i}^{k}=$ $\left.1 / N \operatorname{Tr}\left(\mathscr{A}^{k}\right)\right)$, is equal to the total number of walks of length $k$ from each node of the graph to itself, divided by $N$ [72]. The eigenvalues provide also a series of bounds on the diameter of a graph. As an example, Diam $(G)$ is less than the number of distinct eigenvalues in a generic graph $G$ [16], while, in the case of $k$-regular graphs, i.e. graphs with all degrees equal to $k$, a better estimate of an upper bound can be obtained from the second largest eigenvalue $\mu_{N-1}$, since $\operatorname{Diam}(G) \leqslant \ln (N-1) / \ln \left(k / \mu_{N-1}\right)$.

Important information on the connectivity properties of $G$ can be extracted from the spectra of the normal and the Laplacian matrix. The normal matrix, is defined as $\mathscr{N}=\mathscr{D}^{-1} \mathscr{A}$, where $\mathscr{D}$ is the diagonal matrix with elements $D_{i i}=\sum_{j} a_{i j}=k_{i}$. The combinatorial Laplacian matrix $\Lambda$, also known as Kirchhoff matrix (since it was originally proposed by Kirchhoff), is defined as $\Lambda=\mathscr{D}-\mathscr{A} . \Lambda$ is a symmetric positive semi-definite matrix, because it can be expressed in term of the incidence matrix as $\Lambda=\mathscr{B}^{\mathrm{t}}$ [15]. Consequently, all eigenvalues of $\Lambda$ are real and non-negative, and $\Lambda$ has a full set of $N$ real and orthogonal eigenvectors. Since all rows of $\Lambda$ sum to zero, $\Lambda$ always admits the lowest eigenvalue $\lambda_{1}=0$, with corresponding eigenvector $\mathbf{v}_{1}=(1,1, \ldots, 1)$. It is easy to prove that the multiplicity of the eigenvalue zero equals the number of components of $G$. In general $\lambda_{2}$ (the second smallest eigenvalue) is far from trivial, and a series of theorems from spectral graph theory prove that the larger $\lambda_{2}$ is, the more difficult is to cut $G$ into pieces [15].

The eigenvalues and eigenvectors of $\mathscr{A}, \Lambda$ and $\mathscr{N}$ have been used to characterize either models and real networks [72,73,76-78], and also for discovering the presence of cohesive subgroups and other local features, as will be discussed in details in Section 7.1.1. See Ref. [74] for a general discussion on the role of spectral methods for analyzing and visualizing networks.

\subsection{Topology of real networks}

Many systems in nature and in technology are made by a large number of highly interconnected dynamical units [79]. Coupled biological and chemical systems, neural networks, social interacting species, the Internet or the World Wide Web, are only a few such examples. The first approach to capture the global properties of such systems is to model them as graphs whose nodes represent the dynamical units (for instance the neurons in the brain or the individuals in a social system) and the links stand for the interactions between the units. Of course, this is a very strong approximation, since it means translating the interaction between two dynamical units, which is usually depending on time, space and many more other details, into a simple binary number: the existence or not of a link between the two corresponding nodes. Nevertheless, in many cases of practical interest, such an approximation provides a simple but still very informative representation of the entire system.

During the last decade, the grown availability of large databases, the optimized rating of computing facilities, as well as the development of powerful and reliable data analysis tools, have constituted a better and better machinery to explore the topological properties of several networked systems from the real world. This has allowed to study the topology of 
the interactions in a large variety of systems as diverse as communication $[8,25,80]$, social $[28,81-84]$ and biological systems [85-88]. The main outcome of this activity has been to reveal that, despite the inherent differences, most of the real networks are characterized by the same topological properties, as for instance relatively small characteristic path lengths, high clustering coefficients, fat tailed shapes in the degree distributions, degree correlations, and the presence of motifs and community structures. All these features make real networks radically different from regular lattices and random graphs, the standard models studied in mathematical graph theory. This has led to a large attention towards the understanding of the evolution mechanisms that have shaped the topology of a network, and to the design of new models retaining the most significant properties empirically observed. We shall review various models in Section 2.3. Here, we discuss briefly the most significant topological properties as the small-world effect, degree scale-free distributions, correlations, and the presence of clustering. For a more complete description of some real networks we defer to Chapter 6.

\subsubsection{The small-world property}

The study of several dynamical processes over real networks has pointed out the existence of shortcuts, i.e. bridging links that connect different areas of the networks, thus speeding up the communication among otherwise distant nodes.

In regular hypercubic lattices in $D$ dimensions, the mean number of vertices one has to pass by in order to reach an arbitrarily chosen node, grows with the lattice size as $N^{1 / d}$. Conversely, in most of the real networks, despite of their often large size, there is a relatively short path between any two nodes. This feature is known as the small-world property and is mathematically characterized by an average shortest path length $L$, defined as in Eq. (2.5), that depends at most logarithmically on the network size $N[5,28]$. This property was first investigated, in the social context, by Milgram in the 1960s in a series of experiments to estimate the actual number of steps in a chain of acquaintances [18,89-91].

In its first experiment, Milgram asked randomly selected people in Nebraska to send letters to a distant target individual in Boston, identified only by his name, occupation and rough location. The letters could only be sent to someone whom the current holder knew by first name, and who was presumably closer to the final recipient. Milgram kept track of the paths followed by the letters and of the demographic characteristics of their handlers. Although the common guess was that it might take hundreds of these steps for the letters to reach their final destination, Milgram's surprising result was that the number of links needed to reach the target person had an average value of just six. More recently, a similar experiment conducted by Dodds et al. [92] on e-mail exchanges successfully reproduced Milgram's experiment, but capitalizing on the globalization of the Internet. The e-mail passing messages, indeed, completed enough chains as to allow for their through statistical characterization.

The small-world property has been observed in a variety of other real networks, including biological and technological ones [5,28,40,52], and is an obvious mathematical property in some network models, as for instance in random graphs. At variance with random graphs, the small-world property in real networks is often associated with the presence of clustering, denoted by high values of the clustering coefficient, defined as in Eq. (2.10) (see Table 2.1). For this reason, Watts and Strogatz, in their seminal paper, have proposed to define small-world networks as those networks having both a small value of $L$, like random graphs, and a high clustering coefficient $C$, like regular lattices [28]. In the efficiency-based formalism, such a definition corresponds to networks having a high value of global efficiency $E_{\text {glob }}$, defined as in Eq. (2.6), and a high value of local efficiency $E_{\text {loc }}$, defined as in Eq. (2.11), i.e. to networks extremely efficient in exchanging information both at a global and at a local scale $[30,31]$.

\subsubsection{Scale-free degree distributions}

The usual case in Science until a few years ago was that of homogeneous networks. Homogeneity in the interaction structure means that almost all nodes are topologically equivalent, like in regular lattices or in random graphs. In these latter ones, for instance, each of the $N(N-1) / 2$ possible links is present with equal probability, and thus the degree distribution is binomial or Poisson in the limit of large graph size (see Section 2.3.1). It is not startling then that, when the scientists approached the study of real networks from the available databases, it was considered reasonable to find degree distributions localized around an average value, with a well-defined average of quadratic fluctuations. In contrast with all the expectancies, it was found that most of the real networks display power law shaped degree distribution $P(k) \sim A k^{-\gamma}$, with exponents varying in the range $2<\gamma<3$. The average degree $\langle k\rangle$ in such networks is therefore 
Table 2.1

Basic characteristics of a number of information/communication, biological and social networks from the real-world

\begin{tabular}{|c|c|c|c|c|c|c|c|}
\hline Network & $N$ & $\langle k\rangle$ & $L$ & $C$ & $\gamma$ & $v$ & Ref. \\
\hline AS2001 & 11,174 & 4.19 & 3.62 & 0.24 & 2.38 & $<0$ & {$[8,25,80]$} \\
\hline Routers & 228,263 & 2.80 & 9.5 & 0.03 & 2.18 & $>0$ & {$[8,25,80]$} \\
\hline Gnutella & 709 & 3.6 & 4.3 & 0.014 & 2.19 & $<0$ & {$[100]$} \\
\hline WWW & $\sim 2 \times 10^{8}$ & 7.5 & 16 & 0.11 & $2.1 / 2.7$ & Unknown & [101] \\
\hline Protein & 2,115 & 6.80 & 2.12 & 0.07 & 2.4 & $<0$ & [86] \\
\hline Metabolic & 778 & 3.2 & 7.40 & 0.7 & $2.2 / 2.1$ & $<0$ & [85] \\
\hline Math1999 & 57,516 & 5.00 & 8.46 & 0.15 & 2.47 & $>0$ & {$[81,82]$} \\
\hline Actors & 225,226 & 61 & 3.65 & 0.79 & 2.3 & $>0$ & {$[28,83]$} \\
\hline e-mail & 59,812 & 2.88 & 4.95 & 0.03 & $1.5 / 2.0$ & Unknown & [84] \\
\hline
\end{tabular}

The quantities measured are: number of vertices $N$, characteristic path length $L$, clustering coefficient $C$, average degree $\langle k\rangle$, exponent of the degree distribution $\gamma$, and type of correlations. All networks, except the WWW, metabolic (Escherichia coli) and e-mail networks, are undirected. The two values of $\gamma$ represent, respectively, the in/out-degree exponents when the network is directed.

well defined and bounded, while the variance $\sigma^{2}=\left\langle k^{2}\right\rangle-\langle k\rangle^{2}$ is dominated by the second moment of the distribution that diverges with the upper integration limit $k_{\max }$ as

$$
\left\langle k^{2}\right\rangle=\int_{k_{\min }}^{k_{\max }} k^{2} P(k) \sim k_{\max }^{3-\gamma} .
$$

Such networks have been named scale-free networks [2,93], because power-laws have the property of having the same functional form at all scales. In fact, power-laws are the only functional form $f(x)$ that remains unchanged, apart from a multiplicative factor, under a rescaling of the independent variable $x$, being the only solution to the equation $f(\alpha x)=\beta f(x)$. Power-laws have a particular role in statistical physics because of their connections to phase transitions [94] and fractals [95]. In the following, when referring to scale-free networks, we will denote the class of graphs with power-laws in the degree distribution. Of course, this does not necessarily implies that such graphs are scale-free with respect to other measurable structural properties [96]. These networks, having a highly inhomogeneous degree distribution, result in the simultaneous presence of a few nodes (the hubs) linked to many other nodes, and a large number of poorly connected elements.

In finite-size networks, fat-tailed degree distributions have natural cut-offs [83]. When analyzing real networks, it may happen that the data have a rather strong intrinsic noise due to the finiteness of the sampling. Therefore, when the size of the system is small and the degree distribution $P(k)$ is heavy-tailed, it is sometimes advisable to measure the cumulative degree distribution $P_{\text {cum }}(k)$, defined as $P_{\text {cum }}(k)=\sum_{k^{\prime}=k}^{\infty} P\left(k^{\prime}\right)$. Indeed, when summing up the original distribution $P(k)$, the statistical fluctuations generally present in the tails of the distribution are smoothed. Consequently, if $P(k) \sim k^{-\gamma}$, the exponent $\gamma$ can be obtained from $P_{\text {cum }}(k)$ as one plus the slope of $P_{\text {cum }}(k)$ in a $\log -\log$ plot, i.e., $\gamma=1+\gamma_{\text {cum }}$. Another possibility is that of performing an exponential binning of data [8].

\subsubsection{Some examples}

In Table 2.1 we report the main topological properties of a number of published networks, belonging to three different categories: information/communication, biological and social systems. More details on these and other real networks can be found in Chapter 6 .

The first two information/communication networks considered are two examples of the Internet $[8,25,80]$. In both networks, the nodes are the hosts and the links represent the physical connections among them. AS2001 stands for the Internet at the autonomous system (AS) level as on April 16th, 2001 [97], while Routers indicate the router level graph representation of the Internet [98]. Gnutella is a peer-to-peer [99] network provided by Clip2 Distributed Search Solutions [100], and is an example of networks that are becoming popular as a way to connect thousands of computers that allow sharing of files (for instance, music or video) between users over local and wide-area networks. Finally, the World Wide Web (WWW) is a network formed by the hyperlinks between different Web pages, and, with more than $10^{8}$ nodes, is the largest network ever studied. Differently from the previous ones, this network is directed: each Web page 


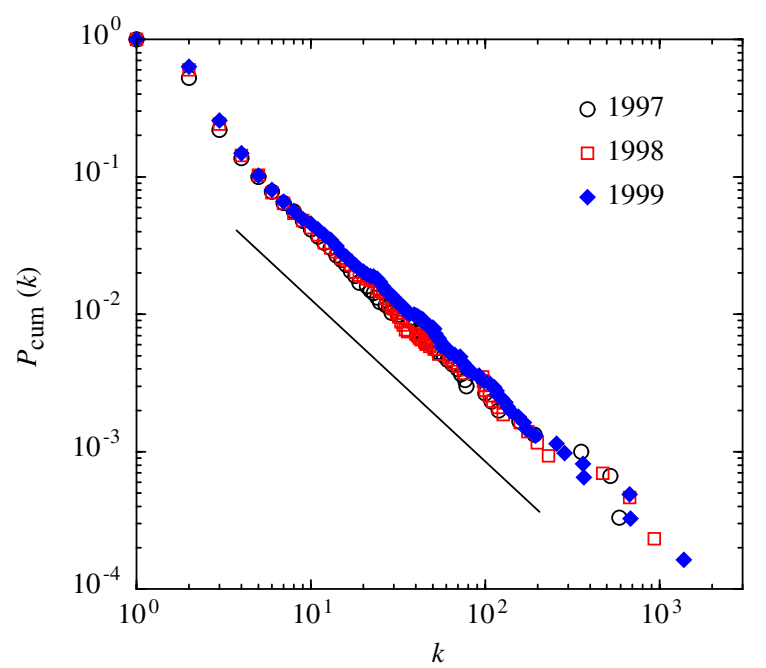

Fig. 2.4. Cumulative degree distributions of the Internet AS graph representation for three different years. The power-law behavior is clear, as well as the fact that, regardless of the very dynamic nature of the Internet, the exponent $\gamma$ is constant with time. Reprinted figure with permission from Ref. [25]. (C) 2001 by the American Physical Society.

has a number of incoming links and a number of outgoing links pointing to other Web pages. All these graphs are sparse with a small average degree $\langle k\rangle$. They all show the small-world property, having a characteristic path length very small as compared to their sizes. They have high values of $C$, and are characterized by power law degree distributions with the exponents reported in table, i.e., they are scale-free. Fig. 2.4 is an example of the cumulative degree distribution of the map of Internet at the autonomous system level for three different years. As it can be noticed, although the Internet is a dynamic entity, in the sense that new nodes are continuously appearing and others are removed, the scale-freeness property of the network is conserved. The graphs considered differ, however, in their degree correlations between nearest neighbor vertices. The AS and Gnutella exhibit disassortative degree correlations, with a tendency to have connections between vertices with dissimilar degrees, as demonstrated by the signs of $v$ reported in table. In contrast, the Router graph displays assortative degree correlations, with a tendency to establish connections between vertices with similar degrees. Recently, it has been pointed out the importance, for some applications involving technological networks, of the degree-degree correlations between nodes at a distance $d \geqslant 1$ [102]. Such correlations can be characterized by reporting the average degree of distance- $d$ neighbors, $\left\langle K^{(d)}\right\rangle_{k}$, restricted to root vertices with degree $k$, as a function of $k$. The case $d=1$ describes the usual nearest neighbors degree correlations. In Fig. 2.5a we plot $\left\langle K^{(d)}\right\rangle_{k}$ for the Internet at the AS level, while in Fig. 2.5b we consider the Routers network. In the first case, the average degree of distance- $d$ neighbors $\left[\left\langle K^{(d)}\right\rangle_{k}\right.$ ] follows, for $d>1$, the same trend as $\left\langle K^{(1)}\right\rangle_{k}$, tending to be less correlated for larger $d$ (see also the inset of Fig. 2.5a where the slope $v$ is reported as a function of $d$ ). In Fig. 2.5b, instead, the degree correlations are assortative up to $d=2$, becoming disassortative for $d>2$. Finally, for $d>6$ the degree correlations in the network show a similar trend than in the disassortative graphs.

The second part of Table 2.1 shows the main topological properties of two biological networks, the protein-protein interaction network in the yeast [86], and a network of metabolic reactions [85,103]. The nodes of the first network are proteins, with two nodes being linked together by an edge if the corresponding proteins physically interact, e.g. if two amino acid chains are binding to each other. This network exhibits a highly inhomogeneous topology similar to those observed in technological networks, with most of the proteins involved in just a few interactions and a few of them interacting with many others. In particular, the probability $P(k)$ of a protein to have $k$ interactions can be fitted by a power law with an exponential cut-off. A similar scale-free behavior has been also reported for the protein-protein interaction network of the bacterium Helicobacter pylori [104]. Because of their potential payoffs, protein-protein interactions and gene networks have been thoroughly studied during the last years [86,105-108], and is now believed that studies such as the one described above could help in the search for specific drugs, or to complete the map of protein functions [108,109]. Metabolic reaction networks are directed networks whose nodes are chemicals that are connected to one another through the existence of metabolic reactions [85,103]. Also in this case, the number of 

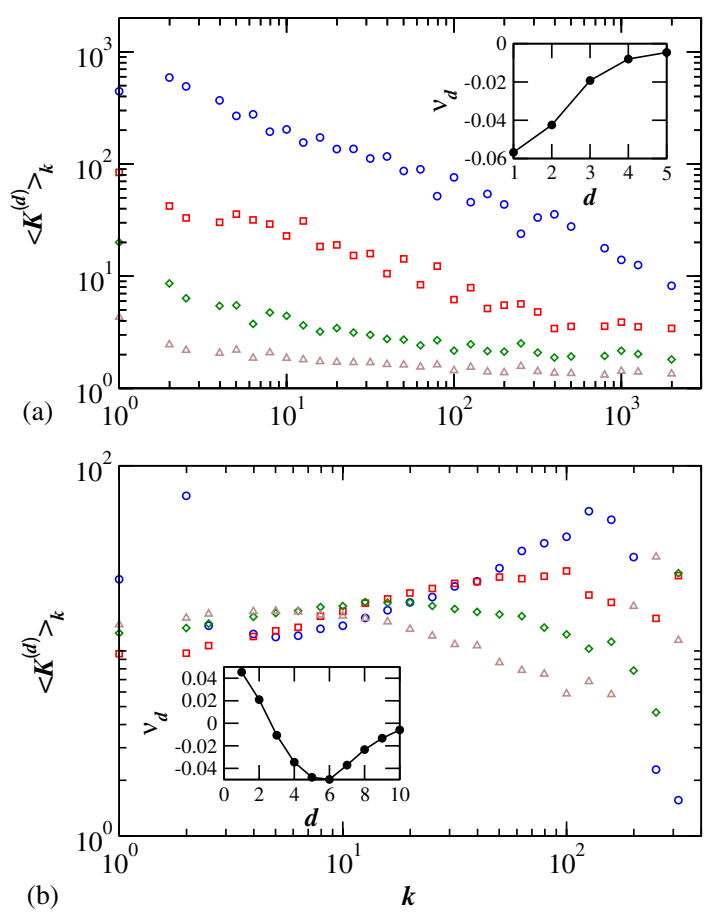

(b)

Fig. 2.5. Average degree of the $d$-nearest neighbors of a root node with degree $k$ in: Internet AS networks (a), and Internet Routers (b). The insets show in both plots the dependency of the slopes of the curves in the main panel as a function of $d$, indicating that the correlations change as the distance from the root is increased. Reprinted with permission from Ref. [102]. (C) 2005 by the American Physical Society.

substrates participating in $k$ metabolic reactions follows a power law with similar exponents both in the in-degree and in the out-degree. Surprisingly, this feature is universal, in the sense that the scale-free character is the same irrespective of the domain of life considered [85]. Metabolic networks have the additional attraction of being highly hierarchical and modular [103]. In particular, the modularity (i.e. the existence of a set of connected molecules that work together to achieve a function) seems to be a fundamental design attribute. We will come back to metabolic, proteins and genetic networks in Section 6.3. Evidences of highly heterogeneous networks can be also found at a higher level of biological organization. A paradigmatic example are food webs. In such networks, nodes represent species in an ecosystem and the directed links stand for the predator-prey relationships. Statistical studies of the topology of several food webs available [87,88,110-113] have not ultimately determined whether or not all food webs can be considered as scale-free networks. However, all studies have concluded that such networks possess the smallworld property and are at least highly inhomogeneous, with a strong cut-off perhaps due to the very small sizes of the ecosystems analyzed.

Finally, we report in Table 2.1 three examples of social networks. Historically, social systems were among the first ones to be studied from a network perspective [89,114]. A social network is formally defined as a set of individuals or social entities linked through some kind of interactions among them. The interactions could be as diverse as friendships, collaborations, sexual contacts or business relationships. Here we consider the collaborations graph of mathematicians defined by paper coauthorships, the movie actor collaboration network based on the Internet Movie Database (a network made up of actors that have casted together in a same movie), and a network of e-mail exchanges [84]. In the latter network the nodes represent e-mail addresses and the directed links stand for messages sent from one address to another. Though the network works through a communication network (the Internet), it is usually considered as a social network since it reflects different kinds of social relationship between end-users. As shown in table, the three networks are all characterized by a power-law in the degree distribution, small values of $L$ and high clustering coefficients. Regardless of the inaccuracy, often associated to the data collection process, many concepts of modern network theory have been drawn from sociometry $[18,19]$ and the study of social networks has spurred recently, since such systems are a good playground for new concepts and tools. Algorithms for detecting community structures, for instance, are usually tested 
in social networks (see Section 7.1), due to natural tendency of such systems to have community structures and cliques of diverse sizes.

\subsection{Networks models}

The observations described in the examples of Section 2.2 have clearly motivated the introduction of new concepts and models. In this section, we focus on the mathematical modelling of networks, discussing some simple and generic models from the point of view of their motivation, construction procedure and significant properties. Further details on models can be found in Refs. [2-4,7].

\subsubsection{Random graphs}

The systematic study of random graphs was initiated by Erdös and Rényi in 1959 with the original purpose of studying, by means of probabilistic methods, the properties of graphs as a function of the increasing number of random connections. The term random graph refers to the disordered nature of the arrangement of links between different nodes. In their first article, Erdös and Rényi proposed a model to generate random graphs with $N$ nodes and $K$ links, that we will henceforth call Erdös and Rényi (ER) random graphs and denote as $G_{N, K}^{\mathrm{ER}}$. Starting with $N$ disconnected nodes, ER random graphs are generated by connecting couples of randomly selected nodes, prohibiting multiple connections, until the number of edges equals $K$ [115]. We emphasize that a given graph is only one outcome of the many possible realizations, an element of the statistical ensemble of all possible combinations of connections. For the complete description of $G_{N, K}^{\mathrm{ER}}$ one would need to describe the entire statistical ensemble of possible realizations, that is, in the matricial representation, the ensemble of adjacency matrices [116]. An alternative model for ER random graphs consists in connecting each couple of nodes with a probability $0<p<1$. This procedure defines a different ensemble, denoted as $G_{N, p}^{\mathrm{ER}}$ and containing graphs with different number of links: graphs with $K$ links will appear in the ensemble with a probability $p^{K}(1-p)^{N(N-1) / 2-K}[14,115,117]$. The two models have a strong analogy, respectively, with the canonical and grand canonical ensembles in statistical mechanics [118], and coincide in the limit of large $N$ [16]. Notice that the limit $N \rightarrow \infty$ is taken at fixed $\langle k\rangle$, which corresponds to fixing $2 K / N$ in the first model and $p(N-1)$ in the second one. Although the first model seems to be more pertinent to applications, analytical calculations are easier and usually are performed in the second model.

ER random graphs are the best studied among graph models, although they do not reproduce most of the properties of real networks discussed in Section 2.2. The structural properties of ER random graphs vary as a function of $p$ showing, in particular, a dramatic change at a critical probability $p_{\mathrm{c}}=\frac{1}{N}$, corresponding to a critical average degree $\langle k\rangle_{c}=1$. Erdös and Rényi proved that $[14,16]$ :

- if $p<p_{\mathrm{c}}$, then almost surely, i.e. with probability tending to one as $N$ tends to infinity, the graph has no component of size greater than $\mathcal{O}(\ln N)$, and no component has more than one cycle;

- if $p=p_{\mathrm{c}}$, then almost surely the largest component has size $\mathcal{O}\left(N^{2 / 3}\right)$;

- if $p>p_{\mathrm{c}}$, the graph has a component of $\mathcal{O}(N)$ with a number $\mathcal{O}(N)$ of cycles, and no other component has more than $\mathcal{O}(\ln N)$ nodes and more than one cycle.

The transition at $p_{\mathrm{c}}$ has the typical features of a second order phase transition. In particular, if one considers as order parameter the size of the largest component, the transition falls in the same universality class as that of the mean field percolation transitions. Erdös and Rényi studied the distribution of the minimum and maximum degree in a random graph [115], while the full degree distribution was derived later by Bollobás [119]. The probability that a node $i$ has $k=k_{i}$ edges is the binomial distribution $P\left(k_{i}=k\right)=C_{N-1}^{k} p^{k}(1-p)^{N-1-k}$, where $p^{k}$ is the probability for the existence of $k$ edges, $(1-p)^{N-1-k}$ is the probability for the absence of the remaining $N-1-k$ edges, and $C_{N-1}^{k}=\left(\begin{array}{c}N-1 \\ k\end{array}\right)$ is the number of different ways of selecting the end points of the $k$ edges. Since all the nodes in a random graph are statistically equivalent, each of them has the same distribution, and the probability that a node chosen uniformly at random has degree $k$ has the same form as $P\left(k_{i}=k\right)$. For large $N$, and fixed $\langle k\rangle$, the degree distribution is well approximated by a Poisson distribution:

$$
P(k)=\mathrm{e}^{-\langle k\rangle} \frac{\langle k\rangle^{k}}{k !} .
$$


For this reason, ER graphs are sometimes called Poisson random graphs. ER random graphs are, by definition, uncorrelated graphs, since the edges are connected to nodes regardless of their degree. Consequently, $P\left(k^{\prime} \mid k\right)$ and $k_{n n}(k)$ are independent of $k$.

Concerning the properties of connectedness, when $p \geqslant \ln N / N$, almost any graph in the ensemble $G_{N, p}^{\mathrm{ER}}$ is totally connected [115], and the diameter varies in a small range of values around Diam $=\ln N / \ln (p N)=\ln N / \ln \langle k\rangle$ $[14,120]$. The average shortest path length $L$ has the same behavior as a function of $N$ as the diameter, $L \sim \ln N / \ln \langle k\rangle$ $[5,14,28,53]$. The clustering coefficient of $G^{\mathrm{ER}}$ is equal to $C=p=\langle k\rangle / N$, since $p$ is the probability of having a link between any two nodes in the graph and, consequently, there will be $p k(k-1) / 2$ edges among the neighbors of a node with degree $k$, out of a maximum possible number of $k(k-1) / 2$ [28]. Hence, ER random graphs have a vanishing $C$ in the limit of large system size.

For large $N$ and $p>p_{\mathrm{c}}$, the bulk of the spectral density of ER random graphs converges to the distribution [2,72]:

$$
\rho(\mu)= \begin{cases}\frac{\sqrt{4 N p(1-p)-\lambda^{2}}}{2 \pi N p(1-p)} & \text { if }|\mu|<2 \sqrt{N p(1-p)}, \\ 0 & \text { otherwise } .\end{cases}
$$

This is in agreement with the prediction of the Wigner's semicircle law for symmetric uncorrelated random matrices [78]. The largest eigenvalue $\left(\mu_{N}\right)$ is isolated from the bulk of the spectrum, and it increases with the network size as $p N$. For $p<p_{\mathrm{c}}$, the spectral density deviates from the semicircle law, and its odd moments $M_{2 k+1}$ are equal to zero, indicating that the only way to return back to the original node is traversing each edge an even number of times.

\subsubsection{Generalized random graphs}

The ER models can be extended in a variety of ways to make random graphs a better representation of real networks. In particular, one of the simplest properties to include is a non-Poisson degree distribution. Random graphs with an arbitrary degree distribution $P(k)$ have been discussed a number of times in the literature.

The configuration model introduced by Bender and Canfield [121] allows to sample graphs with a given degree sequence [122,123]. A degree sequence is any sequence of $N$ integer numbers $D=\left\{k_{1}, k_{2}, \ldots, k_{N}\right\}$ such that $\sum_{i} k_{i}=2 K$, where $K$ is the number of links in the graph. In the configuration model $D$ is chosen in such a way that the fraction of vertices with degree $k$ will tend, for large $N$, to the desired degree distribution $P(k)$. The model considers the ensemble (denoted as $G_{N, D}^{\text {conf }}$ ) of all graphs with $N$ nodes and a given degree sequence $D$, each graph being considered with equal probability. Random configurations on the fixed degree sequence $D$ can be generated by assigning to each node $i$ a number of half-edges equal to its expected degree $k_{i}$, and by forming edges by pairing at random, with uniform probability, two half-edges together. This procedure generates, with equal probability, each possible graph compatible with $D$ [6,122]. In fact, each configuration can be obtained in $\prod_{i}\left(k_{i} !\right)$ different ways, since $k_{i}$ ! are the permutations of the $k_{i}$ indistinguishable half-edges of node $i$. Notice that $G_{N, K}^{\mathrm{ER}}$ can be obtained as a particular case of $G_{N, D}^{\text {conf }}$. A different method, that produces multigraphs, possibly with loops, can be found in Refs. [122,123].

The simplicity of the configuration model makes it a good playground for analytical approaches. Molloy and Reed proved that a giant component emerges almost surely in random graphs with a given $P(k)$ when

$$
Q=\sum_{k} k(k-2) P(k)>0,
$$

and the maximum degree $k_{\max }$ in the graph is not too large. Conversely, when $Q<0$, and $k_{\max }$ is not too large, then almost surely the size of the largest component is $\mathcal{O}\left(k_{\max }^{2} \ln N\right)$ [122]. We shall discuss the derivation of this condition in Section 3.1.2, and the application to the case in which $P(k)$ is a power law in Section 2.3.4. For ER random graphs, formula (2.17) yields the critical average degree $\langle k\rangle_{\mathrm{c}}=1$ discussed in Section 2.3.1. The same authors have studied in Ref. [123] the size of the giant component and the structure of the graph formed by deleting such component.

Newman et al. have proposed a slightly different method to generate graphs with a given degree distribution, in which the degree of nodes are independent identically distributed random integers drawn from a given $P(k)$. In this way, not a single degree sequence, but an ensemble of degree sequences is considered, and the statistical properties of the ensemble, as component sizes and number of nodes at a distance $m$ from a given node, can be calculated by the use of a powerful formalism based on probability generating functions $[4,124]$. Newman et al. have found an approximate 
formula for the average shortest path length that, when $N \ll z_{1}$ and $z_{2} \ll z_{1}$, reads as

$$
L=\frac{\ln \left(N / z_{1}\right)}{\ln \left(z_{2} / z_{1}\right)}+1,
$$

where $z_{m}$ is the average number of neighbors at distance $m$. This formula reduces to the expression discussed in Section 2.3.1, in the special case of ER random graphs, for which $z_{1}=\langle k\rangle$, and $z_{2}=\langle k\rangle^{2}$. More recently, Chung and Lu proposed a model for random graphs with given expected degree sequence and provided a more rigorous proof that $L$ is almost surely of the order of $\ln N / \ln \tilde{d}$, with $\tilde{d}$ equal to the weighted average of the sum of squares of degrees [125].

The clustering coefficient in the configuration model is given by $[4,6,126]$

$$
C=\frac{\langle k\rangle}{N}\left[\frac{\left\langle k^{2}\right\rangle-\langle k\rangle}{\langle k\rangle^{2}}\right]^{2}=\frac{\langle k\rangle}{N}\left[\left(\frac{\sigma_{k}}{\langle k\rangle}\right)^{2}+\frac{\langle k\rangle-1}{\langle k\rangle}\right]^{2},
$$

that is the value for the ER random graphs times an extra factor that can be quite large since its leading term goes as the fourth power of $\sigma_{k} /\langle k\rangle$. Consequently, $C$ vanishes for $N \rightarrow \infty$, although it can be not negligible for highly skewed degree distributions and finite graph sizes as those observed empirically (see Section 2.3.4). Generalization to include clustering properties in random graphs have been less explored in the literature [6,59].

\subsubsection{Small-world networks}

The Watts and Strogatz (WS) model is a method to construct graphs, denoted as $G_{N, K}^{\mathrm{WS}}$ having both the small-world property and a high clustering coefficient [28]. The model is based on a rewiring procedure of the edges implemented with a probability $p$. The starting point is a $N$ nodes ring, in which each node is symmetrically connected to its $2 m$ nearest neighbors for a total of $K=m N$ edges. Then, for every node, each link connected to a clockwise neighbor is rewired to a randomly chosen node with a probability $p$, and preserved with a probability $1-p$. Notice that for $p=0$ we have a regular lattice, while for $p=1$ the model produces a random graph with the constraint that each node has a minimum connectivity $k_{\min }=m$. For intermediate values of $p$ the procedure generates graphs with the small-world property and a non-trivial clustering coefficient. Alternative procedures for constructing small-world networks, based on adding edges instead of rewiring, have also been proposed [126-128].

The richness of the WS model has stimulated an intense activity aimed at understanding the network's properties as a function of the rewiring probability $p$ and the network size $N$ [53,128-130]. As observed in [28], the small-world property results from the immediate drop in $L(p)$ as soon as $p$ is slightly larger than zero. This is because the rewiring of links creates long-range edges (shortcuts) that connects otherwise distant nodes. The effect of the rewiring procedure is highly nonlinear on $L$, and not only affects the nearest neighbors structure, but it also opens new shortest paths to the next-nearest neighbors and so on. Conversely, an edge redirected from a clustered neighborhood to another node has, at most, a linear effect on $C$. That is, the transition from a linear to a logarithmic behavior in $L(p)$ is faster than the one associated with the clustering coefficient $C(p)$. This leads to the appearance of a region of small (but non-zero) values of $p$, where one has both small path lengths and high clustering.

The change in $L(p)$ soon stimulated numerical and analytical work [53,128,129], aimed at inspecting whether the transition to the small-world regime takes place at a finite value of $p$, or if there is a crossover phenomenon at any finite value of $N$ with the transition occurring at $p=0$. This latter scenario turned out to be the case. To see this, we follow the arguments by Barrat and Weigt [53], and Newman and Watts [128]. We assume that $p$ is kept fixed and we inspect the dependency of $L(N, p)$. For small system sizes, the number of shortcuts is less than 1 on average, and the scaling of $L(N, p)$ is linear with the system size. However, for larger values of $N$, the average number of shortcuts eventually becomes greater than one and $L(N, p)$ starts scaling as $\log (N)$. Similar to the correlation length behavior in conventional statistical physics, at some intermediate system value $N=L$, where the transition occurs, we expect $L \sim p^{-\tau}$. Additionally, close to the transition point, $L(N, p)$ should obey the finite-size scaling relation $[53,128]$ :

$$
L(N, p) \sim p^{-\tau} f\left(N p^{\tau}\right),
$$

where $f(x)$ is a universal scaling function that obeys to: $f(x) \sim x$ if $x \ll 1$ and $f(x) \sim \ln x$ if $x \gg 1$. Let us suppose now that $\tau<1$ and assume $\tau<\alpha<1$. From Eq. (2.20) it follows that

$$
L\left(N, N^{-1 / \alpha}\right) \sim N^{\tau / \alpha} f\left(N^{1-\tau / \alpha}\right) \sim N^{\tau / \alpha} \ln \left(N^{1-\tau / \alpha}\right)
$$


as $N^{1-\tau / \alpha} \gg 1$. On the other hand, the average number of rewired connections is $m N^{1-1 / \alpha}$, which vanishes as $N$ grows. From here, Barrat and Weigt deduced that $\tau \geqslant 1$, a result also supported by their numerical simulations. Newman and Watts corroborated this result by a renormalization group analysis and showed that in fact $\tau=1$ [128]. In summary, the model undergoes a continuous phase transition as the density of shortcuts tends to zero with a characteristic length diverging as $p^{-1}$. The exact form of the scaling function $f(x)$ has been obtained in Refs. [129,131].

Barrat and Weigt have obtained a simple formula that fits well the dependency of $C(p)$ observed in the numerical simulations of the WS model [53]. The formula is based on the fact that for $p=0, C(0)=3(m-1) / 2(2 m-1)$. Then, because of the fact that with probability $(1-p)$ edges are not rewired, two neighbors that were linked together at $p=0$ will remain connected with probability $(1-p)^{3}$ up to corrections of order $1 / N$. From here, we get:

$$
C(p) \sim \tilde{C}(p)=\frac{3(m-1)}{2(2 m-1)}(1-p)^{3},
$$

where $\tilde{C}(p)$ is redefined as the ratio between the average number of edges between the neighbors of a vertex and the average number of possible links between the neighbors of a vertex.

As for the degree distribution, when $p=0$ it is a delta function positioned at $2 m$, while for $p=1$ it is similar to that of an ER-network. For intermediate $p$, the degree distribution is given by [53]:

$$
P(k)=\sum_{i=0}^{\min (k-m, m)}\left(\begin{array}{c}
m \\
i
\end{array}\right)(1-p)^{i} p^{m-i} \frac{(p m)^{k-m-i}}{(k-m-i) !} \mathrm{e}^{-p m}
$$

for $k \geqslant m$, and is equal to zero for $k$ smaller than $m$.

Finally, Farkas et al. have studied numerically the properties of the spectra in the SW model, showing that the distribution continuously approaches that of a random graph, as $p$ increases [72], and Monasson has studied the spectral properties of the Laplacian operator both numerically and analytically [127].

\subsubsection{Static scale-free networks}

The large amount of works on the characterization of the topological properties of real networks has motivated the need to construct graphs with power law degree distributions. Graphs with a power-law degree distribution can be simply obtained as a special case of the random graphs with a given degree distribution discussed in Section 2.3.2. We denote such graphs as static scale-free to distinguish them from models of evolving graphs that will be discussed in Section 2.3.5.

Aiello et al. have studied a model that involves only two parameters, $\alpha$ and $\gamma$, and that assigns uniform probability to all random graphs having a number of nodes with degree $k$ given by $N(k)=\mathrm{e}^{\alpha} k^{-\gamma}$ [132]. We denote this model as $G_{\alpha, \gamma}$, since both the total number of nodes $N$ and edges $K$ are fully determined, once $\alpha$ and $\gamma$ are given. Notice that $\alpha$ is the logarithm of the number of nodes with $k=1$, while $\mathrm{e}^{\alpha / \gamma}$ is the maximum degree of the graph. Aiello et al. have shown that the Molloy and Reed criterium predicts that $G_{\alpha, \gamma}$ has a giant component when $\gamma<\gamma_{\mathrm{c}}=3.47875 \ldots$, and have proved that the second largest components are of size $\mathcal{O}(\log N)$ for $2<\gamma<\gamma_{\mathrm{c}}$ and $\mathcal{O}(1)$ for $1<\gamma<2$, while the graph is almost surely connected for $0<\gamma<1$.

By using Eq. (2.19), Newman has calculated the clustering coefficient for power-law degree distributions finding: $C \sim N^{(3 \gamma-7) /(\gamma-1)}$. Thus, $C$ tends to zero in large graphs with $\gamma>7 / 3$, while for $\gamma<7 / 3$, $C$ increases with the system size, due to the fact that there can be, in average, more than one edge between two nodes sharing a common neighbor [4].

Chung and Lu have studied the average distance in random graphs with a power-law degree distribution, proving that $L$ scales as $\log N$ if $\gamma>3$. Conversely, if $2<\gamma<3, L$ is $\mathcal{O}(\log \log N)$, while the diameter is $\mathcal{O}(\log N)$ [125]. Similar results were obtained by Cohen and Havlin [133]. More recently, Chung et al. have shown that the spectrum of the adjacency matrix of random power law graphs follows a power law distribution, while the eigenvalues of the normalized Laplacian follow the semicircle law [134].

Several other recipes to construct static scale-free networks, based on assuming that a node has some intrinsic properties, have been proposed in the physics community. In the model by Goh et al. [42], each node $i$ is assigned a weight (or fitness) $w_{i}=i^{-\alpha}$, where $i=1,2, \ldots, N$ is the node index and $\alpha$ is a tunable parameter in $[0,1$ ). Two different nodes, $i$ and $j$, are selected with probabilities equal to the normalized weights $w_{i} / \sum_{l} w_{l}$ and $w_{j} / \sum_{l} w_{l}$, respectively, and are connected if there is not already a link between them. The process is repeated until $m N$ links are made in the 
system, so that $\langle k\rangle=2 m$. When $\alpha=0$ one obtains a ER random graph. When $\alpha \neq 0$, the graph obtained has a power law degree distribution $P(k) \sim k^{-\gamma}$ with an exponent $\gamma=1+1 / \alpha$. Thus, by varying $\alpha$ in $[0,1)$ one obtains an exponent $\gamma$ in the range $2<\gamma<\infty$. The betweenness distribution follows a power law with an exponent $\delta=2.2$ for any value of $\gamma$ in the range $2<\gamma \leqslant 3[42,45]$.

A similar fitness model was studied by Caldarelli et al. [135]. The model starts with $N$ isolated nodes, and associates at every node $i$ a fitness $\eta_{i}$, which is a real number taken from a fitness distribution $\rho(\eta)$. For every couple of nodes, $i$ and $j$, a link is drawn with a probability $f\left(\eta_{i}, \eta_{j}\right)$, with $f$ being a symmetric function of its arguments. The model generates power-law $P(k)$ for various fitness distributions and attaching rules, while it gives ER random graph if $f\left(\eta_{i}, \eta_{j}\right)=p \forall i, j$. Recently, Masuda et al. focused on the threshold graph model which is a subclass of the previous model particularly suited to the analytical treatment. The connectivity between a pair of nodes in the model is determined by whether the sum of the fitness of the pair exceeds a given threshold $\theta$, namely $f\left(\eta_{i}, \eta_{j}\right)=1$ if $\eta_{i}+\eta_{j} \geqslant \theta$, and $f\left(\eta_{i}, \eta_{j}\right)=0$ otherwise [136].

We finally mention gradient networks, i.e. directed graphs induced by local gradients of a scalar field distributed on the nodes [137-139]. In the simplest model, Toroczkai and Bassler consider a given substrate network, $S$, and a non-degenerate scalar, $h_{i}$, associated with each node $i$ and describing the potential of the node. Then, the gradient network is constructed as the collection of directed links pointing from each node to whichever of its neighbors on $S$ has the highest potential [137]. It can be shown that gradient networks consist only of trees. Furthermore, if the substrate network $S$ is scale-free, and the scalars $h_{i}$ are independent identically distributed random variables, then the associated gradient network is scale-free and characterized by the same exponent. More interestingly, if $S$ is a random graph (and $h_{i}$ are independent identically distributed random variables), then the gradient network is scale free with the in-degree distribution $P\left(k^{i n}\right)$ being a decreasing power law with an exponent $\gamma_{i n}=1[137,138]$.

\subsubsection{Evolving scale-free networks}

Static scale-free graphs are good models for all cases in which growth or aging processes do not play a dominant role in determining the structural properties of the network. Conversely, there are many examples of real networks in which the structural changes are ruled by the dynamical evolution of the system. Here we discuss a class of models whose primary goal is to reproduce the growth processes taking place in real networks: the rationale is that, by mimicking the dynamical mechanisms that assembled the network, one will be able to reproduce the topological properties of the system as we see them today. We concentrate primarily on the model of network growth proposed by Barabási and Albert in 1999, and on its various variants.

The Barabási-Albert (BA) model is a model of network growth inspired to the formation of the World Wide Web and is based on two basic ingredients: growth and preferential attachment [93]. The basic idea is that in the World Wide Web, sites with high degrees acquire new links at higher rates than low-degree nodes. More precisely, a undirected graph $G_{N, K}^{\mathrm{BA}}$ is constructed as follows. Starting with $m_{0}$ isolated nodes, at each time step $t=1,2,3, \ldots, N-m_{0}$ a new node $j$ with $m \leqslant m_{0}$ links is added to the network. The probability that a link will connect $j$ to an existing node $i$ is linearly proportional to the actual degree of $i$ :

$$
\prod_{j \rightarrow i}=\frac{k_{i}}{\sum_{l} k_{l}} .
$$

Because every new node has $m$ links, the network at time $t$ will have $N=m_{0}+t$ nodes and $K=m t$ links, corresponding to an average degree $\langle k\rangle=2 \mathrm{~m}$ for large times. The BA model has many similarities with a model developed by Price [140] in 1976 to explain the power laws that the same author found, one decade earlier, in citation networks (both for the in-degree and the out-degree distributions) [141]. In Price's model, the probability that a new published paper cites a previous one is taken to be proportional to $k_{i n}+1$, where $k_{i n}$ is the number of times that the paper has already been cited. Price's model is a reformulation, in terms of network growth, of a model developed by Simon in 1955 to explain the power laws that appear in a wide range of empirical data, as in the distribution of words in prose samples by their frequency of occurrence, or in the distributions of cities by population [142] (see Ref. [143] for an application of the Simon's model to the growth of the World Wide Web). The detailed discussion on the solution of Price's model can be found in the review by Newman [4]. Here, we simply mention that the model has two main differences with respect to the BA model: it builds a directed graph, and the number of edges added with each new node is not a fixed quantity. 
The BA model has been solved in the mean-field approximation $[93,144]$ and, exactly, by means of rate equation [145] and master equation [146] approaches. In the limit $t \rightarrow \infty$, the model produces a degree distribution $P(k) \sim k^{-\gamma}$, with an exponent $\gamma=3$. The case of a growing network with a constant attachment probability $\prod_{j \rightarrow i}=1 /\left(m_{0}+t-1\right)$ produces, instead, a degree distribution $P(k)=e / m \exp (-k / m)$. This implies that the preferential attachment is an essential ingredient of the model.

Krapivsky and Redner have considered a directed version of the BA model. They have studied the relation between the age $a$ of a node $i$, defined as the number of nodes added after node $i$, and its degree, and the correlations in the degree of adjacent nodes [147]. They found that the number of nodes of age $a$ with $k$ links, $N_{a}(k)$, is equal to

$$
N_{a}(k)=\sqrt{1-\frac{a}{N}}\left(1-\sqrt{1-\frac{a}{N}}\right)^{k} .
$$

Thus, the degree distribution for nodes of fixed age decays exponentially with the degree, with a characteristic degree scale which diverges as $(1-a / N)^{-1 / 2}$ for $a \rightarrow N$. The same authors have also shown that there are correlations in the degrees of neighboring nodes, so that nodes of similar degree are more likely to be connected [147] in sharp contrast to the standard BA model where degree-degree correlations are absent. An alternative method to generate degree correlations in a BA scale-free network (or in a generic undirected uncorrelated network) has been proposed in Ref. [148]. The method is based on the following iterative algorithm: at each step two links of the network are chosen at random, and the four end-nodes are ordered with respect to their degrees; then, with a probability $p$, the links are rewired in such a way that one link connects the two nodes with the smaller degrees and the other connects the two nodes with the larger degrees while, with a probability $1-p$, the links are randomly rewired; in the case when one or both of these new links already existed in the network, the step is discarded. A repeated application of the rewiring step leads to an assortative version of the original network without changing the degree distribution of the network. Changing the parameter $p$, it is possible to construct networks with different degree of assortativity.

The average distance in the BA model is smaller than in a ER-random graph with same $N$ and $K$, and increases logarithmically with $N$ [2]. Analytical results predict a double logarithmic correction to the logarithmic dependence $L \sim \log N / \log (\log N)$ [149]. The clustering coefficient vanishes with the system size as $C \sim N^{-0.75}$. This is a slower decay than that observed for random graphs, $C \sim N^{-1}$, but it is still different from the behavior in small-world models, where $C$ is a constant independent of $N$.

The graph spectrum in the BA model has a triangle-like shape different from the circular distribution observed in ER-random graphs. In the central region $\rho(\mu)$ decreases exponentially, while it shows a power-law decay on the tails $[72,73]$. The largest eigenvalue $\mu_{N}$ depends on the system size as $N^{1 / 4}$ for large $N$, and the corresponding eigenvector is strongly localized on the node with the largest degree [73].

The BA model has attracted an exceptional amount of attention in the literature. In addition to analytic and numerical studies of the model itself, many authors have proposed modifications and generalizations to make the model a more realistic representation of real networks [2]. Various generalizations, such as models with nonlinear preferential attachment, with dynamic edge rewiring, fitness models and hierarchically and deterministically growing models, can be found in the literature. Such models yield a more flexible value of the exponent $\gamma$ which is restricted to $\gamma=3$ in the original BA construction. Furthermore, modifications to reinforce the clustering property, which the BA model lacks, have also been considered. Here we will discuss a few of these models.

The Dorogovtsev-Mendes-Samukhin (DMS) model considers a linear preferential attachment of the form

$$
\prod_{j \rightarrow i}=\frac{k_{i}+k_{0}}{\sum_{l}\left(k_{l}+k_{0}\right)}
$$

with $-m<k_{0}<\infty$ [146]. This is a more general form than Eq.(2.23), because of the presence of the constant $k_{0}$, that plays the role of the node initial attractiveness. For such attachment probability one gets a power law degree distribution with an exponent $\gamma=3+k_{0} / m$. Hence, as the initial attractiveness $k_{0}$ grows from $-m$ to $\infty, \gamma$ increases from 2 to $\infty$. For $k_{0}=0$ one gets the BA model $[4,7,146]$. 
Krapivsky et al. have studied a model with a nonlinear attachment probability of the form

$$
\prod_{j \rightarrow i}=\frac{k_{i}^{\alpha}}{\sum_{l} k_{l}^{\alpha}},
$$

proving that the linearity is a necessary condition in order to get a power law degree distribution. Indeed, if $\alpha<1$, the rule (2.26) produces a stretched exponential degree distribution, while, for $\alpha>1$, a single site connects to nearly all other sites [145,147].

A model with accelerating growth, in which the number of new edges $m$ added at each time step increases with the network size as $m \sim N^{\alpha}(t)$, has been proposed in Ref. [150].

Preferential attachment rules with fluctuations have been considered in Refs. [151,152], while Gardenes and Moreno have studied to which extent the global character of the preferential attachment rule is crucial in the BA-model, by considering a preferential attachment rule that applies only to the neighborhood of the newly added nodes [153].

The BA model does not allow for changes after the network is formed. However, in real networks like the WWW or a social network, connections can be lost and added. Albert and Barabási (AB) have proposed a generalization of their original model, based on the three following rules [154]. At each time step, with probability $p$, one selects an existing node and adds $m$ new links from it to $m$ nodes chosen preferentially. Then, with probability $q$, one rewires $m$ arbitrarily selected edges. Finally, with probability $1-p-q$, one adds a new node to the network and connects it with $m$ existing nodes preferentially. The model shows a regime in which $P(k)$ is a power law with an exponent whose value is determined by $p$ and $q$.

Dorogovtsev and Mendes [155], Tadic [156,157], Krapivsky et al. [158] and other authors [159-162] have studied similar models in which the edges can appear and disappear, or they can move around the network after they are added. Such models show either power-law and exponential degree distributions depending on the parameters.

In real networks, relatively young nodes can attract many connections because of their own intrinsic properties. Examples are well summarized new Web pages that receive more links than older nodes, or interesting recent scientific articles being more cited than older ones. The Bianconi-Barabási (BB) model incorporates the intrinsic fitness of a node into the rules of the original BA model. The BB model is based on the following attachment probability $[163,164]$ :

$$
\prod_{j \rightarrow i}=\frac{\eta_{i} k_{i}}{\sum_{l} \eta_{l} k_{l}} .
$$

Here $\eta_{i}$ is the fitness of node $i$, drawn from a given distribution $\rho(\eta)$. In the particular case in which $\rho(\eta)$ is constant and $\eta$ is confined in $[0,1]$ one gets $P(k) \sim k^{-2.26} / \ln (k)$ and degree correlations, namely $k_{n n} \sim k^{-v}$ with $v>0$. Bianconi and Barabási also showed an interesting connection between evolving networks and an equilibrium Bose gas, when the fitness $\eta_{i}$ is replaced by $\epsilon_{i}=-\beta^{-1} \log \eta_{i}$, with $\beta$ playing the role of inverse of temperature [164].

The Ravasz-Barabási (RB) model aims at reproducing the hierarchical organization observed in some real networks [165]. The model combines scale-free properties with a high degree of clustering, and is based on iteratively repeating the same construction rules at different scales. Details and properties of the RB model will be discussed in Section 6.2 in relation to the modelling of the Internet topology. Hierarchically and deterministically growing models have been discussed also in Refs. [103,166-168].

Generalizations to reinforce the clustering properties, lacking in the BA model, have also been considered in the literature. The simplest solution is to embed a triangle-generating protocol [126] into the BA model [169-171]. The Holme-Kim (HK) model consists in adding new nodes each with $m$ links, as in the BA model, and connecting them either to a neighbor of a previously connected node or by using the usual preferential attachment rule. The HK model produces scale-free degree distributions and a clustering coefficient that can be varied up to 0.5 for $m=3$ [169].

The Jin-Girvan-Newman model generates a high clustering coefficient based on three simple mechanism inspired to the dynamics of social networks [171]: (i) two individuals with a common friend have a high probability to meet each other; (ii) relationships between individuals having few meetings decrease with time; (iii) an individual has a limited capacity for relationships.

The introduction of node deactivation procedures also enhances clustering [172,173]. The Klemm-Eguiluz (KE) model, also known as structured scale-free model, describes the growth dynamics of a network with directed links based on a finite memory of the nodes [172]. Each node of the network can be in two different states: active or inactive. The model starts with a complete graph of $m$ active nodes. At each time step, a new node $j$ with $m$ outgoing links is 
added. Each of the $m$ active nodes receives one incoming link from $j$. The new node $j$ is then activated, while one of the $m$ active nodes is deactivated. The probability $\prod_{i}^{\text {deact }}$ that node $i$ is deactivated is given by

$$
\prod_{i}^{\text {deact }}=\frac{1}{k_{i}+a}\left(\sum_{l \in \mathcal{N}_{\mathrm{act}}} \frac{1}{k_{l}+a}\right)^{-1},
$$

where $k_{i}$ is the in-degree of node $i, a$ is a positive constant and the summation runs over the set $\mathscr{N}_{\text {act }}$ of the currently active nodes. The procedure is iteratively repeated until the desired network size is reached. The model produces a scale-free network with $\gamma=2+a / m$ and with a clustering coefficient larger than in the BA model, e.g. $C=5 / 6$ when $a=m$. However, the network does not show the small-world property, as the characteristic path length is proportional to the network size $(L \sim N)$ [173]. The additional rewiring of edges may recover the small-world property [174]. It is also worth noting that the outcome of the procedure sketched above strongly depends on the order in which deactivation and addition of nodes are performed [173].

A different class of models is that of evolving networks inspired to protein interactions. The Solé-Pastor Satorras-Smith-Kepler (SPSK) model is based on three mechanisms [175]: (i) duplication (an arbitrarily selected node is duplicated and its connections are also duplicated), (ii) divergence (connections of a duplicated node are removed with a give probability $\delta$ ), (iii) mutation (connections are added from the duplicated node to $\alpha N$ nodes which are not neighbors of the original node). The SPSK model produces power law degree distribution; however the degree correlations and the clustering coefficient do not show realistic values [176].

The Vázquez-Flammini-Maritan-Vespignani (VFMV) model includes duplication and divergence in a different way [177]. Precisely, duplication is implemented by generating a duplicate $A^{\prime}$ from a randomly selected node $A$, and connecting $A$ and $A^{\prime}$ with a probability $p$. Divergence is implemented by deleting with probability $q$ one of the connections from a common neighbor $(B)$ of $A$ and $A^{\prime}$. This model shows a high clustering because of the triangle formed by $A, A^{\prime}$ and $B$. By choosing proper values of $q, k_{n n}(k)$ results a decreasing function of $k$, as in real networks. However the size of the giant cluster is relatively smaller than that of a real network. Other similar models were proposed by Berg et al. [178], Karev et al. [179], and Goh et al. [180].

We finally mention that scale-free topologies can also be obtained as a result of optimization processes (see for instance Refs. [181,182]). This opens up an alternative (to model growing graphs) and promising methods that still need to be investigated in much depth.

\subsection{Weighted networks}

Up to now, we have mostly focussed our discussion on unweighted networks, i.e. networks that have a binary nature, where the edges between nodes are either present or not. Nevertheless, along with a complex topological structure, many real networks display a large heterogeneity in the capacity and the intensity of the connections. Examples are the existence of strong and weak ties between individuals in social networks $[29,30,52,183,184]$, uneven fluxes in metabolic reaction pathways [185,186], the diversity of the predator-prey interactions in food webs [187-190], different capabilities of transmitting electric signals in neural networks [30,31,191,192], unequal traffic on the Internet [8] or of the passengers in airline networks $[49,193,194]$. These systems can be better described in terms of weighted networks, i.e. networks in which each link carries a numerical value measuring the strength of the connection.

Here, we present a set of measures that allow for a statistical characterization of weighted networks. In particular, we briefly review some applications to cases from the real world. The empirical results demonstrate that purely topological models are inadequate to explain the rich and complex properties observed in real systems, and that there is a need for models to go beyond pure topology. We discuss some of the models recently proposed for weighted networks and which naturally produce the observed broad scale distributions and topology-weight correlations. The main dynamical consequences of a weighting procedure on edges will be discussed in Chapter 5 .

\subsubsection{Measures for weighted networks}

A weighted (or valued) graph $G^{\mathrm{W}}=(\mathscr{N}, \mathscr{L}, \mathscr{W})$ consists of a set $\mathscr{N}=\left\{n_{1}, n_{2}, \ldots, n_{N}\right\}$ of nodes (or vertices, or points), a set $\mathscr{L}=\left\{l_{1}, l_{2}, \ldots, l_{K}\right\}$ of links (or edges, or lines), and a set of values (weights) $\mathscr{W}=\left\{w_{1}, w_{2}, \ldots, w_{K}\right\}$ that are real numbers attached to the links. The number of elements in $\mathscr{N}$ and $\mathscr{L}$ are denoted by $N$ and $K$, 
respectively. A weighted graph can be drawn as in Fig. 2.1(c), with the thicknesses of the links representing their weights. In matricial representation, $G^{\mathrm{W}}$ is usually described by the so-called weights matrix $\mathscr{W}$, a $N \times N$ matrix whose entry $w_{i j}$ is the weight of the link connecting node $i$ to node $j$, and $w_{i j}=0$ if the nodes $i$ and $j$ are not connected ( $w_{i i}=0 \forall i$, unless explicitly mentioned). In the following, we consider only the case of positive and symmetric weights $w_{i j}=w_{j i} \geqslant 0$, while asymmetrically weighted networks will be treated specifically in Chapter 5, due to their relevance in enhancing synchronization phenomena. Negative weights are occasionally appropriate, e.g. they can represent animosity between individuals in a social network; see for instance Ref. [195]. As for unweighted graphs, $\mathscr{A}$ represents the underlying $N \times N$ adjacency matrix such that $a_{i j}=1$ if $w_{i j} \neq 0$, and $a_{i j}=0$ if $w_{i j}=0$. A first characterization of a weighted graph can be obtained by the weight distribution $Q(w)$, i.e. from the probability that a given edge has weight $w$. Following is a list of other useful quantities that generalize and complement concepts already introduced for unweighted networks, and that combine weighted and topological observables [196].

1. Node strength, strength distributions and correlations. In a weighted graph, the natural generalization of the degree $k_{i}$ of a node $i$ is the node strength (or node weight, or node weighted connectivity) $s_{i}$, defined as [194,197,198]:

$$
s_{i}=\sum_{j \in \mathscr{N}} w_{i j} .
$$

The strengths integrate the information on the number (degree) and the weights of links incident in a node. When the weights are independent on the topology, the strength of the vertices of degree $k$ is $s(k) \simeq\langle w\rangle k$ where $\langle w\rangle$ is the average weight. In the presence of correlations we obtain in general $s(k) \simeq A k^{\beta}$ with $\beta=1$ and $A \neq\langle w\rangle$ or $\beta>1$ (see Section 2.4.2).

For a given node $i$, with connectivity $k_{i}$ and strength $s_{i}$, different situations can arise. All weights $w_{i j}$ can be of the same order $s_{i} / k_{i}$ or, in contrast, only one or a few weights can dominate over all the others. The disparity in the weights of a node $i$ can been evaluated [196,199] by the quantity $Y_{i}$ defined as [200,201]

$$
Y_{i}=\sum_{j \in \mathscr{N}_{i}}\left[\frac{w_{i j}}{s_{i}}\right]^{2},
$$

where $\mathscr{N}_{i}$ is the set of first neighbors of $i$, as in formula (2.3). By definition, $Y_{i}$ has an implicit dependence on $k_{i}$. If all edges have comparable weights, then $Y(k)$ (i.e. the disparity averaged over all nodes with degree $k$ ) scales as $1 / k$. If, however, the weight of a single link dominates Eq. (2.30), then $Y(k) \simeq 1$; in other words $Y(k)$ is independent of $k$ [199].

The strength distribution $R(s)$ measures the probability that a vertex has strength $s$, and, altogether with the degree distribution $P(k)$, provides useful information on a weighted network. In particular, since the node strength is related to the node degree, it is expected to observe heavy-tailed $R(s)$ distributions in weighted networks with slow decaying $P(k)$. Similarly to Eq. (2.3), a weighted average nearest neighbors degree of a node $i$ can be defined as [194]

$$
k_{n n, i}^{\mathrm{W}}=\frac{1}{s_{i}} \sum_{j \in \mathcal{N}} a_{i j} w_{i j} k_{j} .
$$

This is the local weighted average of the nearest neighbor degree, according to the normalized weight of the connecting edges $w_{i j} / s_{i}$. Such a quantity allows to characterize the assortative/disassortative behavior in a weighted network. Indeed, $k_{n n, i}^{\mathrm{W}}>k_{n n, i}$ when the edges with the larger weights are pointing to the neighbors with larger degree, and $k_{n n, i}^{\mathrm{W}}<k_{n n, i}$ in the opposite case. The $k_{n n, i}^{\mathrm{W}}$ function thus measures the effective affinity to connect neighbors with high or low degree according to the magnitude of the actual interactions. Similarly, the behavior of the function $k_{n n}^{\mathrm{W}}(k)$ (defined as the average of $k_{n n, i}^{\mathrm{W}}$ over all vertices with degree $k$ ) marks the weighted assortative or disassortative properties considering the actual interactions among the system's elements.

2. Weighted shortest paths. In some cases, it is useful to imagine a physical length associated to each of the edges. In the case of networks embedded in $D$-dimensional Euclidean space (usually $R^{2}$ or $R^{3}$ ), the length $\ell_{i j}$ of the 
edge $l_{i j}$ can be identified with the Euclidean distance between the positions of node $i$ and $j$. In a generic weighted network, the length of the edge can be introduced as some function of the weight. For instance it could be taken $\ell_{i j}=1 / w_{i j}$, although such an assumption does not respect the triangle inequality [30,31,52]. In a weighted network, the path with the minimum number of edges is not necessarily an optimal one. It is then possible to define the weighted shortest path length $d_{i j}$ as the smallest sum of the edge lengths throughout all the possible paths in the graph from $i$ to $j$. See Refs. [30,52,202] and references therein for the algorithms to find weighted shortest paths. The definition of $L, E$ and $b_{i}$, given in Section 2.1.2, can be extended by considering weighted shortest paths. In the particular case of unweighted graphs we assume $\ell_{i j}=1$ for all edges, and the weighted shortest path length $d_{i j}$ reduces to the minimum number of edges traversed to get from $i$ to $j$ (See Section 2.1).

3. Weighted clustering coefficient. The clustering coefficient as defined in Section 2.1.3 does not take into account the fact that, in a weighted network, some neighbors are more important than others. In Ref. [194], Barrat et al. have defined the weighted clustering coefficient of a given node $i$ as:

$$
c_{i}^{\mathrm{W}}=\frac{1}{s_{i}\left(k_{i}-1\right)} \sum_{j, m} \frac{\left(w_{i j}+w_{i m}\right)}{2} a_{i j} a_{j m} a_{m i} .
$$

An alternative definition, taking into account the three (instead of only two) link weights of a triangle, can be found in Ref. [203]. The definition in Eq. (2.32) reduces to the topological node clustering coefficient of formula (2.9), when all the weights are equal. In the general case, the weighted clustering coefficient considers both the number of closed triangles in the neighborhood of node $i$ and their total relative weight with respect to the vertex strength. The factor $s_{i}\left(k_{i}-1\right)$ is a normalization factor and ensures that $0 \leqslant C_{i}^{\mathrm{W}} \leqslant 1$. The quantities $C^{\mathrm{W}}$ and $C^{\mathrm{W}}(k)$ are respectively the weighted node clustering coefficient averaged over all nodes in the graph and over all nodes with degree $k$, respectively. In the case of a large randomized network (lack of correlations) it is easy to see that $C^{\mathrm{W}}=C$ and $C^{\mathrm{W}}(k)=C(k)$.

In real weighted networks, however, we can face two opposite cases. If $C^{\mathrm{W}}>C$, we are in presence of a network in which the interconnected triples are more likely formed by the edges with larger weights. On the contrary, $C^{\mathrm{W}}<C$ signals a network in which the topological clustering is generated by edges with low weight. In this case it is obvious that the clustering has a minor effect in the organization of the network, since the largest part of the interactions (traffic, frequency of the relations, etc.) is occurring on edges not belonging to interconnected triples. The same may happen for $C^{\mathrm{W}}(k)$, for which it is also possible to analyze the variations with respect to the degree class $k$.

4. Motifs. Onnela et al. have recently proposed a systematic way to extend the motifs analysis to a weighted (directed or undirected) graph $G^{\mathrm{W}}=(\mathscr{N}, \mathscr{L}, \mathscr{W})$ [203]. A motif $M$ is here defined as a set of the topological equivalent subgraphs of $G^{\mathrm{W}}$. The intensity $I(g)$ and the coherence $Q(g)$ of a given subgraph $g=\left(\mathscr{N}_{g}, \mathscr{L}_{g}, \mathscr{W}_{g}\right)$ with $n$ nodes and $l$ links are respectively defined as:

$$
I(g)=\left(\prod_{(i j) \in \mathscr{L}_{g}} w_{i j}\right)^{1 / l}, \quad Q(g)=I(g) / \sum_{(i j) \in \mathscr{L}_{g}} w_{i j} .
$$

The intensity $I(g)$ is the geometric mean of the weights in $g$, and is considerably lower in a triangle with two strong and one weak link, than in a triangle with three strong links. Due to the nature of the geometric mean, the subgraph intensity $I(g)$ may be low because one of the weights is very low, or it may result from all of the weights being low. $Q(g)$ allows two to distinguish between these two extremes, being $Q(g) \leqslant 1 \forall g$, and $Q(g) \sim 1$ only if the subgraph weights do not differ too much, i.e. are internally coherent. The intensity of a motif $M$ in $G^{\mathrm{W}}$ is defined as the sum of its subgraph intensities: $I_{M}=\sum_{g \in M} I(g)$. Analogously, the coherence of $M$ is $Q_{M}=\sum_{g \in M} Q(g)$. Replacing the number of subgraphs by their intensities generalizes the $Z$-score defined in Eq.(2.12) to the motif intensity score ZI:

$$
(Z I)_{M}=\frac{I_{M}-\left\langle I_{M}^{\mathrm{rand}}\right\rangle}{\sigma_{I_{M}}^{\mathrm{rand}}},
$$


where $I_{M}$ is the intensity of motif $M$ in $G^{\mathrm{W}}$, while $\left\langle I_{M}^{\text {rand }}\right\rangle$ and $\sigma_{I_{M}}^{\text {rand }}$ are, respectively, mean and standard deviation of intensities in the randomized graph ensemble. In an analogous way the motif coherence score $Z Q$ is defined as

$$
(Z Q)_{M}=\frac{Q_{M}-\left\langle Q_{M}^{\mathrm{rand}}\right\rangle}{\sigma_{Q_{M}}^{\mathrm{rand}}} .
$$

Notice that both $Z I$ and $Z Q$ tend to $Z$ of Eq. (2.12), as the weights become binary.

\subsubsection{Real weighted networks}

Here we present some of the most striking examples of real systems which have been studied as weighted networks. It has been found that the weights characterizing the various connections exhibit complex statistical features with highly varying distributions and power-law behaviors. Correlations between weights and topology provide a complementary perspective on the structural organization of such systems.

Biological networks. It is by now well recognized the important role played by the topology in cellular networks, the intricate web of interactions among genes, proteins and other molecules regulating cell activity $[63,85,86,103,204,205]$. More recently, it has also been shown that further important information resides in the intensity of the interactions. Almaas et al. [199] have studied the metabolic reactions of the E. coli metabolism as a weighted network, with the weights $w_{i j}$ being given by the fluxes from metabolite $i$ to $j$. The fluxes observed are highly inhomogeneous with a coexistence of fluxes spanning several order of magnitude: under optimal growth conditions, the weight (flux) distribution is best fitted with a power law $Q(w) \propto\left(w_{0}+w\right)^{-\gamma_{w}}$ with $w_{0}=0.0003$ and $\gamma_{w}=1.5$. Moreover, it has been found that the average link weight scales with the degrees of the two end-nodes as $\left\langle w_{i j}\right\rangle \sim\left(k_{i} k_{j}\right)^{\theta}$, with an exponent $\theta=0.5$ [206]. The large-scale inhomogeneity observed in the overall flux distribution is also valid at the level of the individual metabolites, as indicated by the calculation of the disparity measure defined in Eq. (2.30). For the E. coli metabolism optimized for succinate and glutamate uptake, it has been found that both the in and out degrees follow $Y(k) \sim k^{-0.27}$. This is an intermediate behavior between the two extreme scaling $Y(k)=$ const and $Y(k) \sim k^{-1}$ and indicates that the larger is the number of reactions that consume (produce) a given metabolite, the more likely it is that a single reaction carries the majority of the flux [199]. The analysis of the weighted motifs of such a network can be found in Ref. [203].

Tieri et al. [207] have studied the communication between cells of the human immune system as a weighted directed graph in which the various cell types are the nodes and the weights $w_{i j}$, associated to each arc, are equal to the number of different soluble mediators secreted by cell $i$ and affecting cell $j$. The results show that immune cells form a highly inhomogeneous network in which only few soluble mediators play a central [37] role in mediating the interactions between the different cell types [207].

Social networks. Collaboration networks are among the most extensive databases of social networks considered to date $[28,29,52,184,185,194,196]$. The topological properties of the graph of film actors and of several scientific collaboration networks have been studied in Refs. [28,52,184,185]. In particular, Newman has shown that scientific collaboration networks have all the general ingredients of small-world and scale-free networks [52,184], while Barabási et al. have followed a complementary approach more focused on the dynamical processes determining the network evolution [185]. In such unweighted approach, scientists are identified with the nodes of the graph and two scientists are linked if they have co-authored at least one paper. On the other hand, authors who have written many papers together are expected to know each other better than those who have co-authored only few papers. To account for this, it is standard to weight ties by the frequency of collaboration [18].

In the physics literature this method has been used by Marchiori et al. [29] to study the network of movie actors. In the case of scientific collaborations, Newman has proposed to define the weight of the interaction between two collaborators $i$ and $j$ as $w_{i j}=\sum_{p} \delta_{i}^{p} \delta_{j}^{p} /\left(n_{p}-1\right)$, where the index $p$ runs over all papers, $\delta_{i}^{p}$ is 1 if author $i$ has contributed to paper $p$ and 0 otherwise, and $n_{p}$ is the number of authors of the paper $p$. The normalization factor $n_{p}-1$ takes into account that the authors of a large collaboration know one another less well, on average, than authors from a smaller collaboration, and implies that the strength $s_{i}$ of node $i$ is equal to the number of papers author $i$ has coauthored with others [52].

By using the definition of weights proposed by Newman, Barrat et al. [194] have considered the connected network of $N=12,722$ scientists who have authored manuscripts submitted to the condensed matter physics e-print archive 


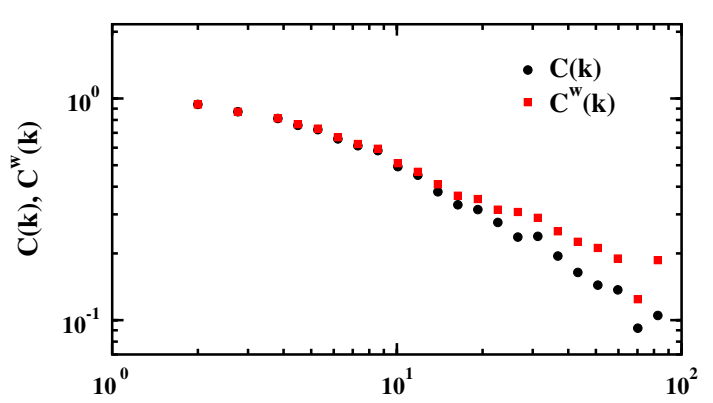

(a)

k (number of collaborators)

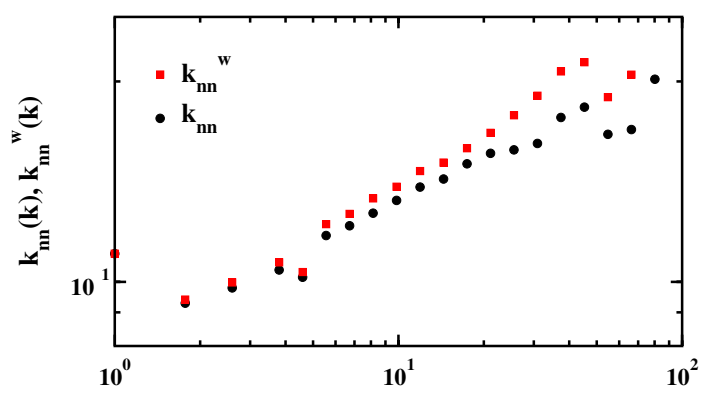

(c)

k (number of collaborators)

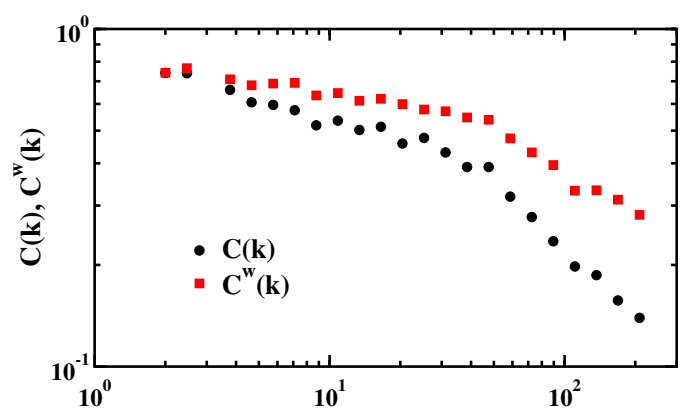

(b)

k (non-stop connections)

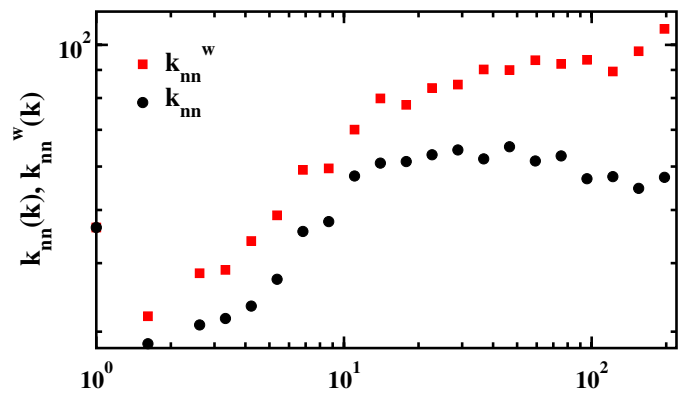

(d)

k (non-stop connections)

Fig. 2.6. From Ref. [194]. Comparison of topological and weighted quantities for the scientific collaborations [panel (a) and (c)], and the world-wide airport network [panel (b) and (d)], considered in Refs. [194,196]. In panel (a) the weighted clustering coefficient $C^{\mathrm{W}}(k)$ separates from the topological one around $k \geqslant 10$, while in panel (b) $C^{\mathrm{W}}(k)$ is larger than $C(k)$ in the whole degree spectrum. In panel (c) the assortative behavior is shown both by the unweighted and the weighted definition of the average nearest neighbors degree. In panel (d), $k_{n n}(k)$ is reaching a plateau for $k>10$ denoting the absence of marked topological correlations while, on the contrary, $k_{n n}^{w}(k)$ exhibits an assortative behavior. Courtesy of A. Vespignani.

[208] between 1995 and 1998. The observed empirical distribution $R(s)$ is heavy tailed, as the degree distribution $P(k)$. The weights are independent from the topology, since the average strength $s(k)$ shows, as a function of the degree $k$ of nodes, the linear behavior $s(k)=\langle w\rangle k$ expected in a random distribution of the actual weights on the existing topology. In (Fig. 2.6) some of the topological and weighted quantities introduced in Sections 2.1 and 2.4.1 are compared. The behavior of $C(k)$ indicates that authors with few collaborators are more probable to work within groups in which all the scientists collaborate together (high clustering) than authors with a large degree. For $k \geqslant 10$ the weighted clustering coefficient $C^{\mathrm{W}}(k)$ is larger than $C(k)$. This implies that authors with many collaborators tend to publish more papers with interconnected groups of co-authors, and has been interpreted as a signature of the fact that influential scientists form stable research groups where the largest part of their papers is produced [194]. The behavior of $k_{n n}(k)$ and $k_{n n}^{\mathrm{W}}(k)$, both growing as a power-law as a function of $k$, indicates that the network considered exhibits the assortative character typical of social networks [26].

Technological networks. Weights are provided naturally by fluxes/traffic related to transportation in critical infrastructure networks such as the Internet, railway, subway and air-flights networks. The topological properties of airplane connection networks have been intensively studied in Refs. [49,193]. In Ref. [194] Barrat et al. have analyzed the world-wide airport network as a weighted graph with the weights $w_{i j}$ being given by the number of available seats on direct flight connections between the airports $i$ and $j$. The topology of graph exhibits both small-world and scale-free properties, as already observed in different databases [49,193]. In particular, the degree distribution takes the form $P(k)=k^{-\gamma} f\left(k / k_{x}\right)$, where $\gamma \simeq 2.0$ and $f\left(k / k_{x}\right)$ is an exponential cut-off function that finds its origin in physical constraints on the maximum number of connections that a single airport can handle $[49,83]$.

The probability that a vertex has strength $s$ is heavy tailed and the weights are non-trivially correlated to the degree: the average link weight scales with the degrees of the two end-nodes as $\left\langle w_{i j}\right\rangle \sim\left(k_{i} k_{j}\right)^{\theta}$, with an exponent $\theta=0.5[194,206]$. The strength of vertices of degree $k$ follows a power-law behavior $s(k) \simeq A k^{\beta}$ with an exponent $\beta=1.5 \neq 1$. This 
means that the larger is an airport, the more traffic it can handle. Concerning clustering coefficients and average nearest neighbor degrees, the weighted analysis provides an even richer scenario than that observed in scientific collaboration network. The weighted clustering coefficient $C^{\mathrm{w}}(k)$ has much more limited variation in the whole spectrum of $k$. This implies that high degree airports have a progressive tendency to form interconnected groups with high traffic links, thus balancing the reduced topological clustering. Since high traffic is associated to hubs, we have a network in which high degree nodes tend to form cliques with nodes with equal or higher degree, the so-called rich-club phenomenon [209]. The topological $k_{n n}(k)$ does show an assortative behavior only at small degrees. For $k>10, k_{n n}(k)$ approaches a constant value, a fact revealing a uncorrelated structure in which vertices with very different degrees have a very similar neighborhood. The analysis of the weighted $k_{n n}^{w}(k)$, however, exhibits a pronounced assortative behavior in the whole $k$ spectrum, providing a different picture in which high degree airports have a larger affinity for other large airports where the major part of the traffic is directed.

\subsubsection{Modelling weighted networks}

The easiest way to construct a weighted network is by considering a random graph with a given probability distribution $P(k)$, and by assuming that the weights of the edges are random independent variables, distributed according to a weight distribution $Q(w)$. See Ref. [210] for the calculation of the resulting node strength distribution $R(s)$ in some examples. A more interesting situation is that in which the evolutions of degrees and weights are coupled. The range of possible models in which the link weights are active variables is extremely broad.

The Yook-Jeong-Barabási-Tu (YJBT) model is a minimal model for weighted scale-free networks in which both the topology and the weights are driven by the connectivity according to the preferential attachment rule as the network grows [197]. The topology of the model is the same as that of the BA model for unweighted scale-free networks. Starting with $m_{0}$ nodes, at each time step a new node $j$ with $m \leqslant m_{0}$ links is added to the network. The probability that a link will connect $j$ to an existing node $i$ is that of formula (2.23). Each link of $j$ is assigned a weight $w_{j i}\left(=w_{i j}\right)$ given by

$$
w_{j i}=\frac{k_{i}}{\sum_{\left\{i^{\prime}\right\}} k_{i^{\prime}}} .
$$

Different weight assignments, as for instance $w_{j i} \propto\left(k_{i} k_{j}\right)^{\theta}, w_{j i} \propto \max \left(k_{i}, k_{j}\right), w_{j i} \propto \min \left(k_{i}, k_{j}\right)$, have been considered in Refs. [206,211]. The YJBT model generates a scale-free network with $P(k) \sim k^{-\gamma}, \gamma=3$, and with a power law strength distribution $R(s) \sim s^{-\gamma_{s}}$, with an exponent $\gamma_{s}$ different from the connectivity exponent, and strongly dependent on $m$. The difference in the exponents is a result of strong logarithmic corrections, and asymptotically (i.e., in the long time limit) the strength distribution converges to the scaling behavior of the degree distribution [197].

The Zheng-Trimper-Zheng-Hui (ZTZH) model is a generalization of the YJBT model incorporating a stochastic scheme for weight assignments based on both the degree and the fitness of a node [212]. Each newly established link $l_{j i}$ is given, with a probability $p$, a weight as in Eq. (2.36), and, with probability $1-p$, a weight $w_{j i}\left(=w_{i j}\right)$ given by

$$
w_{j i}=\frac{\eta_{i}}{\sum_{\left\{i^{\prime}\right\}} \eta_{i^{\prime}}}
$$

where $\eta_{i}$ is a fitness parameter assigned to each node $[163,164]$ and chosen at random from a uniform distribution $\rho(\eta)$ in the interval $[0,1]$. For $p=1$ the model reduces to the YJBT model, while for $p=0$ the weights are driven entirely by the fitness. The model leads to a power-law strength distribution $R(s) \sim s^{-\gamma_{s}}$ characterized by an exponent that is highly sensitive to the probability $p$, and decreases continuously, from the value $\gamma_{s}=3=\gamma$ at $p=0$, as $p$ increases. As in the YJBT model, the difference with respect to the scaling in $P(k)$ is the result of logarithmic correction terms, that can be tuned by $p$ and disappear for $p=0$. The dependence of $\gamma_{s}$ on $m$, found in the YJBT model, persists for all $p>0$. Only when $p=0, \gamma_{s}$ is independent of $m$. Similar results were also found in a model with a link formation mechanism dependent on both node connectivity and fitness [212].

Antal-Krapivsky (AK) have proposed a model for weighted networks in which the structural growth of the network is coupled with the edge weights [213]. The model is defined as follows. At each time step a new node $j$ is added and linked to a single $(m=1)$ target node $i$, with probability proportional to the strength of $i$ :

$$
\prod_{j \rightarrow i}=\frac{s_{i}}{\sum_{l} s_{l}} .
$$




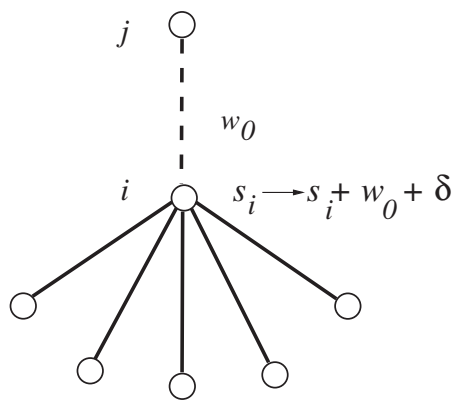

Fig. 2.7. Reprinted figure with permission from Ref. [214] BBV model, (C) 2004 by the American Physical Society. Local rearrangement of weights due to the presence of the new node $j$ and the new link $l_{j i}$. The weight of the new edge is $w_{0}$ and the total weight on the existing edges connected to $i$ is modified by an amount equal to $\delta$.

This rule relaxes the usual degree preferential attachment of formula (2.23), focussing on a strength driven attachment in which new vertices connect more likely to vertices handling larger weights and which are more central in terms of the strength of interactions. This is a plausible mechanism in many real networks: in the Internet new routers connect to more central routers in terms of bandwidth and traffic handling capabilities [8], while in the scientific collaboration network, considered in Section 2.4.2, authors with more co-authored papers are more visible and open to further collaborations. Each attached link is assigned a positive weight which is drawn from a distribution $\rho(w)$. The resulting network is a tree, whose strength distribution $R(s)$ approaches, for long times, a stationary distribution with a universal tail $R(s) \sim s^{-3}$ as $s \rightarrow \infty$, independently on the link weight distribution $\rho(w)$. In particular, when $\rho(w)$ is exponential, $R(s)$ is algebraic over the entire range. Other generalizations to this model, in which the network acquires cycles, give $R(s) \sim s^{-\gamma_{s}}$, with an exponent $\gamma_{s}$ varying from 3 to 2 , as the average node degree increases from 1 to $+\infty$ [213].

The YJBT, ZTZH and AK models discussed above are all based on growing topologies where the weights are assigned to the link when the link is created, and remain fixed thereafter. Such models ignore the possible dynamical evolution of weights occurring when new vertices and edges are introduced in the system. On the other hand, the evolution and reinforcements of interactions is a common feature of natural and infrastructure networks, e.g., in the airplane networks considered in Section 2.4.2, when a new airline connection is created between two airports, it will generally modify the traffic of both airports. The Barrat-Barthélemy-Vespignani (BBV) model is based on a weight-driven dynamics and on a weights' reinforcement mechanism coupled to the local network growth which mimics the change in the interactions observed in real situations. The model dynamics starts from an initial seed of $m_{0}$ vertices connected by links with assigned weight $w_{0}$. At each time step, a new vertex $j$ is added with $m$ edges (with initial weight $w_{0}$ ) that are randomly attached to a previously existing vertex $i$ according to the probability distribution of formula (2.38). The presence of the new link $l_{j i}$ introduces a local rearrangements of weights between $i$ and its neighbors $l$ according to the rule:

$$
w_{i l} \rightarrow w_{i l}+\Delta w_{i l} \quad \forall l \in \mathscr{N}_{i},
$$

where

$$
\Delta w_{i l}=\delta \frac{w_{i l}}{s_{i}} .
$$

This rule considers that the establishment of a new edge of weight $w_{0}$ with the vertex $i$ induces a total increase of traffic $\delta$ that is proportionally distributed among the edges departing from the vertex according to their weights (see Fig. 2.7), yielding $s_{i} \rightarrow s_{i}+\delta+w_{0}$.

The networks generated by the BBV model display power-law behavior for the weight, degree and strength distributions with non-trivial exponents related to $\delta$ and $w_{0}$. Without loss of generality it can be set $w_{0}=1$, so that the model depends on a single parameter $\delta$, that is the fraction of weight which is induced by the new edge onto the others. In the large time limit, one obtains $Q(w) \sim w^{-\gamma_{w}}$ with $\gamma_{w}=2+1 / \delta$ for the distribution of the weights; and a power-law distribution of both the vertex degrees and the vertex strengths, $P(k) \sim k^{-\gamma}$ and $R(s) \sim s^{-\gamma_{s}}$, with the same exponent $\gamma=\gamma_{s}=(4 \delta+3) /(2 \delta+1)[214]$. 


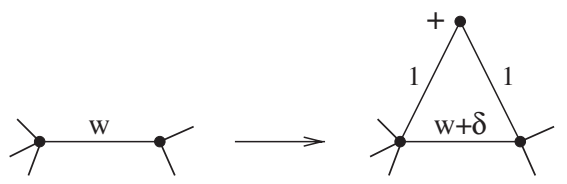

Fig. 2.8. From Ref. [210] DM model. Schematic view of the network growth. At each time step, the weight of a preferentially chosen edge is increased by an amount equal to $\delta$ and a new vertex is attached to the ends of this edge with two edges having weight equal to 1 .

This result indicates that the BBV model produces scale-free networks with an exponent $\gamma \in[2,3]$ : when $\delta=0$ the model is topologically equivalent to the BA model [93] discussed in Section 2.3.5, and $\gamma=3$; for larger values of $\delta$, the power law gets broader, with $\gamma=2$ in the limit $\delta \rightarrow \infty$. Moreover, the model produces correlations between topology and weights. The weight scales as a function of the degree as $s(k) \simeq A k^{\beta}$, with $\beta=1$ and $A \neq\langle w\rangle$ [214], while (weighted and unweighted) average nearest neighbor degree and clustering coefficient (see Section 2.4.3) exhibit different behavior as a function of $k$, depending on the basic parameter $\delta$ [215]. The BBV model can be further generalized in order to include in the coupling the effects of additional randomness and non-linearities which can be present in specific real networks. In particular, cases with a parameter $\delta_{i}$ being a function, either random or related to the specific properties of the node, e.g. $w_{i l}, k_{i}$, and $s_{i}$, have been considered [215-217].

An alternative mechanism leading to results similar to those of the BBV model is the Dorogovtsev-Mendes (DM) model [210]. While in the first model the high strength nodes attract new edges and afterwards the weights of the edges of these nodes are specifically modified, in the DM model high weight edges increase their weights and attract new connections. That is, in one approach, the attachment is to strong vertices and in the other approach, the attachment is to heavy edges. This is a principal difference which can be related to distinct real situations [210] (Fig. 2.8). The rules of the DM model are the following. The growth starts from an arbitrary configuration of nodes and edges, e.g., from a single edge of weight 1 . At each time step: (i) an edge is chosen with a probability proportional to its weight and its weight is increased by a constant $\delta$; (ii) a new vertex is attached to both the ends of this edge by edges of weight 1 . In the particular case of $\delta=0$, the model reduces to the model of Ref. [218]. The distribution of edge weight, node degree and node strength of the resulting network are power-laws, with exponents respectively equal to $\gamma_{w}=2+2 / \delta$ and $\gamma=\gamma_{s}=2+1 /(1+\delta)$.

Some other models and generalization of the previous models can be found in Refs. [195,202,206,211,215-219,220].

There has also been much very recent work on various aspects of weighted networks including characterizing paths on these networks [202] and minimum spanning trees [206], and the application to weighted networks to diverse physical systems, such as earthquakes [221] and market investments [222]. In particular, some aspects related to the dynamic of weighted networks, as epidemic spreading [223,224], and synchronization phenomena will be discussed in the following chapters.

\subsection{Spatial networks}

A particular class of networks are those embedded in the real space, i.e. networks whose nodes occupy a precise position in two or three-dimensional Euclidean space, and whose edges are real physical connections. The typical example are neural networks, in which neural cells are often separated by long distances and, with a few exceptions of well known diffusible mediators, all the communications between neural cells go along physical connections [192]. Other important examples include information/communication networks [8,34], electric power grids [225], transportation systems ranging from river [226], airport [49,194,227], street [47], railway and subway [228] networks, ant networks of galleries [33]. Most of the works in the literature have focused on the characterization of the topological properties of spatial networks, while the spatial aspect has received less attention, when not neglected at all. However, it is not surprising that also the topology of spatial networks is strongly constrained by their geographical embedding. Following is a list of the major characteristics and spatial constraints at work on such networks.

- The first information has to do with the spatial distribution of the nodes of the network. Networks with strong geographical constraints, such as power grids or transport networks, are examples of networks with fractal scaling [229]. E.g., Yook et al. have shown that the nodes of the Internet develop on a fractal support driven by the fractal 


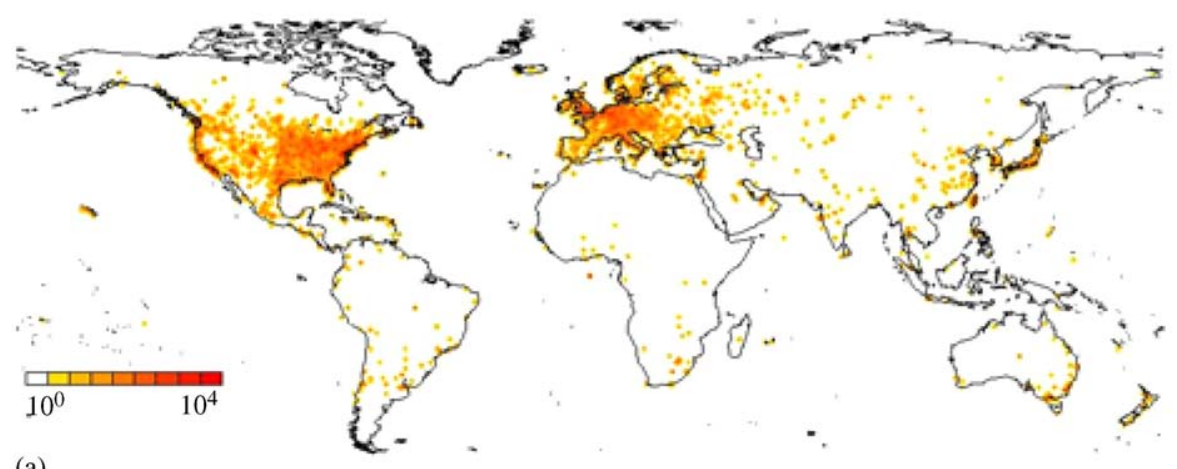

(a)

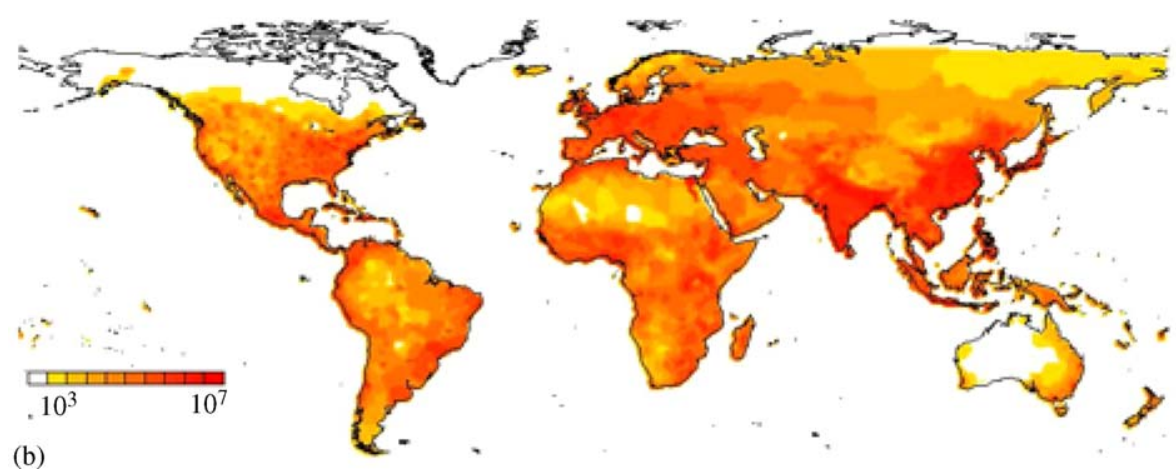

Fig. 2.9. (a) Worldwide router density map obtained by locating the geographical position of the 228265 routers of the router-level Internet database from Ref. [231]; (b) population density map. In either maps the resolution consists of boxes of $1^{\mathrm{o}} \times 1^{\mathrm{O}}$. The color code indicates the density values. The highest population density within this resolution is of the order $10^{7}$ people/box, while the highest router density is of the order of $10^{4}$ routers/box. Figure taken from Ref. [230]. Courtesy of A.L. Barabási.

nature of population patterns around the world [230]. The maps reported in Fig. 2.9 indicate a strong correlations between the worldwide geographical distribution of the Internet routers and the population density in economically developed areas of the world.

Yook et al. have used a box counting method to analyze the spatial distribution of the population density and of the Internet, considered both at the level of routers and at the level of autonomous systems (See Section 6.2). The results, reported in Fig. 2.10(a), indicate that each of the three sets is a fractal with fractal dimension $D_{f}=1.5 \pm 0.1$. The coincidence between the fractal dimension of the population and the Internet (router and AS) nodes is due to the fact that a high population density implies a higher demand for Internet services, resulting in higher router and domain density. Fig. 2.10(b) shows that the router and AS densities increase monotonically with the population density.

- Node degrees are constrained in spatial networks, since the number of edges that can be connected to a single node is limited by the physical space to connect them. This is particularly evident in planar networks, such as street patterns [47] or ant networks of galleries [33]. Planar graphs are those graphs forming vertices whenever two edges cross, whereas non-planar graphs can have edge cross and not form vertices [16]. The maximum number of links that can be accomodated in a planar graph with $N$ nodes is equal to $3 N-6$ [15].

- Two distant nodes are less likely to be connected due to the distance-dependent cost of the edges. The fact that long-range connections are constrained by the Euclidean distance has important consequences on the small-world behavior, as we shall discuss in Section 2.5.1.

- Spatial networks show trivial clustering-degree correlations. Ravasz and Barabási have shown that two geographical networks, the Internet at the router level and the power grid of the Western United States, have $C(k)$ independent of $k$ [165]. This result is different from what has been obtained for many other real networks which show a hierarchical behavior with $C(k)$ well approximated by $C(k) \sim k^{-1}$. In networks with strong geographical constraints, hierarchy is absent because of the limitations imposed by the link lengths on the topology. 

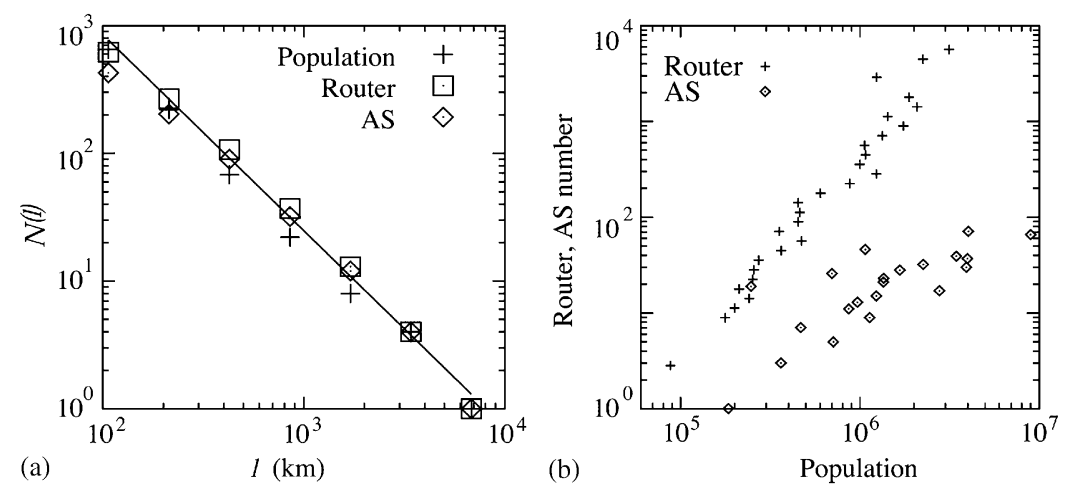

Fig. 2.10. (a) The box counting method [95] applied to the distributions shown in Fig. 6.8. The $\log -\log$ plot shows the number of boxes of size $l \times l$ with non-zero routers/AS/inhabitants as a function of $l$ for North America. The slope of the straight line indicates that $D_{f}=1.5 \pm 0.1$ for each data set. (b) The plot of the number of router/AS nodes in a $1^{\circ} \times 1^{\circ}$ box as a function of the number of people living in the same area, shows that router density is strongly correlated with economic factors. Figure taken from Ref. [230]. Courtesy of A.L. Barabási.

In the following subsections we shall discuss a series of methods, measures and models recently proposed to take into consideration the geographical position of the nodes and the physical lengths of the connections.

\subsubsection{The small-world behavior in Euclidean space}

Stoneham was the first one to investigate the spatial aspects of the small-world problem outlined by Milgram [89] in the context of geography, by examining channelling effects and the sensitivity of the overall structure to disconnection and the ghettisation of an area [232].

In Ref. [233], Csanyi and Szendroi have worked out an alternative definition to characterize the small-world behavior, proving that networks with strong geographical constraints are not small worlds. Since the standard definition (based on the logarithm scaling of the characteristic path length $L$ with $N$ ) is meaningless for networks with a fixed size, the authors have proposed to use $N_{i}(r) \sim \exp (\alpha r)$ as an indication of the small-world scaling, being $N_{i}(r)$ the number of nodes that can be reached from vertex $i$ in at most $r$ steps. Such a scaling is opposed to the fractal scaling $N_{i}(r) \sim r^{D}$ (the network discrete analog of fractal scaling), that is instead found to be valid in many geographical networks, as the Western US power grid, the Hungary water network and the London underground network [96,233].

Gorman and Kulkarni have explicitly encompassed the spatial aspects into the definition of a small world [234]. The fundamental idea behind small-world networks is the notion that the networks gain efficiency by having a large number of local connections and a few global links connecting local clusters together. For such a reason, the authors have proposed to divide the area spanned by the US Internet infrastructures into $m$ regions (with a variable number of nodes inside each region and a variable number of connections between regions) and to measure the so called global connectivity $C_{i(q)}$ of a node $i$ belonging to an area $q$ as the normalized ratio between the number of links from node $i$ to regions outside region $q$ and the number of links from $i$ to other nodes inside the same region $q$. The Internet is non-planar (New York can connect directly to San Francisco bypassing all intervening cities between the two) and this allows for the global links of small-world network to be formed.

Concerning the modelling of small-world networks, in the rewiring process of the WS model two nodes are connected independently of their Euclidean distance [28]. This means that the length distribution of the shortcuts is uniform. A series of models in which this condition is relaxed have been discussed in the literature. In Ref. [5] Watts has considered graphs in which the nodes can connect with either a uniform or a Gaussian probability distribution to other nodes within a finite spatial distance $\xi$. Due to the presence of the finite cutoff $\xi$, spatial graphs of this kind are not small worlds since the characteristic path length and the clustering coefficient show the same functional dependence on the parameter $\xi$. New interesting properties emerge for distributions with no finite cutoff, particularly for those with infinite variance. Networks with a distribution $q(\ell)$ of shortcut lengths $\ell$ decaying as a power law $\left[q(\ell) \sim \ell^{-\alpha}\right]$ have been considered in Refs. [5,235-241]. The networks are constructed by adding a number $p N$ of long range links, with length-dependent probability $q(\ell)$, to a $D$-dimensional lattice made up of $N=L^{D}$ sites and nearest neighbor links. Navigability [235,236] (See Section 7.2), and the nature of random walks over such networks [237], depend on 


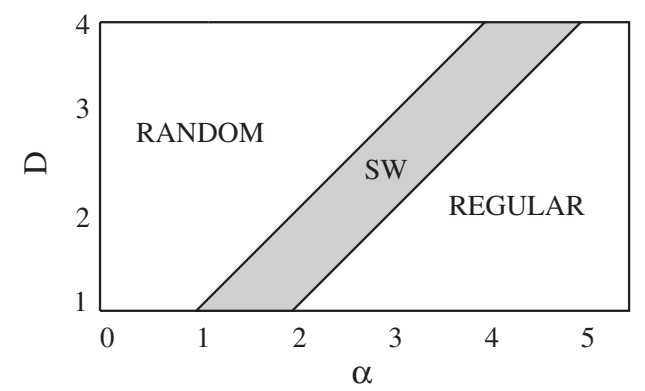

Fig. 2.11. Phase diagram of $D$-dimensional lattices supplemented with long range links whose lengths are distributed according to $q(\ell) \sim \ell^{-\alpha}$. SW denotes the small-world phase in the $\alpha-D$ plane. Reprinted figure with permission from Ref. [240]. C) 2002 by the American Physical Society.

the value of the exponent $\alpha$. Differently from the cases with finite cutoff, a transition to small-world behavior is here expected for $\alpha$ smaller than a critical value, i.e. when a certain amount of long shortcuts is provided. The values of $\alpha$ such that a small-world topology is realized is a matter of debate [238-241].

Jespersen and Blumen have shown that random walks on 1D-networks with $\alpha<2$ have a behavior qualitatively similar to that of walkers over small-world networks, while for $\alpha>2$ they are reminiscent of random walks on regular lattices [237]. Sen et al. have pointed out that three kinds of behavior are expected to be present in these networks or, in other words, there are three regions along the $\alpha$ axis such that, for $\alpha<\alpha_{c}^{(1)}$ the network behaves as a random network, for $\alpha_{c}^{(1)}<\alpha<\alpha_{c}^{(2)}$ it is small-world-like, and for $\alpha>\alpha_{c}^{(2)}$ it is like a regular network [238,240]. In Ref. [240], the authors focus on the first transition in the 1D case. By means of analytical arguments, they are able to prove that, for $\alpha<\alpha_{c}^{(1)}$, with $\alpha_{c}^{(1)}=1$, the clustering coefficient vanishes as $L \rightarrow \infty$, and to determine the characteristic critical exponents of the transition. Moreover, by simple arguments based on the scaling behavior of the average link length $\bar{\ell}$, they conjecture that the two transition points in any dimension are: $\alpha_{c}^{(1)}=D$ and $\alpha_{c}^{(2)}=D+1$ (see Fig. 2.11).

In Ref. [241], Petermann and De Los Rios have studied the distribution of flows over the shortcuts confirming both analytically and numerically (by means of the scaling of distances) that, for networks in $D$ dimensions $\alpha_{\mathrm{c}}^{(2)}=D+1$. This is in agreement with the value $\alpha_{\mathrm{c}}^{(2)}=2$ obtained in $D=1$ by Moukarzel and Argollo de Menezes [239].

\subsubsection{Scale-free networks in Euclidean space}

A series of studies on how to combine the scale-free structure with a precise spatial layout have been proposed in the literature. Here, we discuss growing rules that take into consideration the lengths of the connections or the fact that nodes cannot have unlimited number of links.

Xulvi-Brunet and Sokolov have considered a growing network model with disadvantaged long-range links. At each time step, a new node $j$ is added on a $1 D$ lattice with periodic boundary conditions and linked to $m$ target nodes $i$ with a probability:

$$
\prod_{j \rightarrow i} \sim k_{i} \ell_{i j}^{-\alpha}
$$

where $\ell_{i j}$ is the Euclidean distance between $i$ and $j$, and $\alpha>0$ is a tunable parameter of the model [242]. Numerical simulations have shown that for $\alpha<1$ the degree distribution is a power law $P(k) \sim k^{-3}$ as in the BA model, whereas for $\alpha>1$ it is fitted by a stretched exponential form $P(k)=a \exp \left(-b k^{\gamma}\right)$. On the other hand, the property $L \sim \ln N$ is preserved for all values of $\alpha$. In Ref. [243] Manna and Sen have studied the same model in $D$ dimensions, proving that the resulting link length distribution follows a power law $q(\ell) \sim \ell^{-\delta}$, with an exponent $\delta$ that can be calculated exactly for whole range of values of $\alpha$, being $\delta(\alpha)=\alpha-D+1$ for $\alpha \leqslant 2 D$, and $\delta=D+1$ for $\alpha>2 D$. The same authors have conjectured, by means of scaling arguments, that the transition from scale-free behavior to stretched exponential degree distributions happens at $\alpha_{c}=D-1$. This formula is confirmed by numerical simulation in $2 D$, though it gives a value $\alpha_{c}=0$ in $1 D$ that is different from what found numerically in Ref. [242].

Soares et al. have studied numerically the degree distribution emerging in $2 D$ growing models based on rule (2.41), and where each new site is located at a distance $r$ from the barycenter of the pre-existing graph, according to the probability $1 / r^{2+\alpha_{G}}$ with $\alpha_{G} \geqslant 0$ [244]. 
In Ref. [245], Barthélemy has studied the finite-range case by considering the node randomly distributed with uniform density $\rho$ in a $D$-dimensional space of linear size $L$, and an attachment probability $\prod_{j \rightarrow i} \sim k_{i} \exp \left(-\ell_{i j} / \xi\right)$, i.e. with a term dependent on the spatial distance that is negligible above a finite scale $\xi$. The usual BA scale-free networks are recovered if $\xi>L$, while for $\xi \ll L$ the resulting degree distribution has a cutoff dependent on the density $\rho$. Moreover, when $\xi \ll L$, a finite clustering coefficient $C$ and an assortative behavior are observed [245]. Evolving networks with different functions of the Euclidean distance in the attachment probability, but no dependence on the degree, can be found in Refs. [246,247].

The general problem of embedding a scale-free topology in a Euclidean lattice has been considered in Refs. [248-250]. Rozenfeld et al. have studied the case in which each site of a $D$-dimensional lattice of linear size $L$ is a node with a connectivity taken from a scale-free distribution $P(k)=C k^{-\gamma}$ for $m<k<K$. Each of the nodes is then selected at random and connected to its closest neighbors until its connectivity $k$ is realized, or until all sites up to a distance $r(k)=A k^{1 / D}$, with $A$ being a parameter of the model, have been explored [248]. In this model, sites with a connectivity larger than a certain cutoff $k_{\mathrm{c}} \sim(\gamma-2)^{-1} A^{D}$ (that implies a cutoff length $\xi \sim(\gamma-2)^{-1 / D} A^{2}$ ) cannot be realized because of the saturation of the surrounding sites. Consequently, when $L \rightarrow \infty$, scale-free networks with $\gamma>2$ can be successfully embedded up to an Euclidean distance $\xi$, which can be made as large as desired upon the changing of $A$. When the lattice is finite, the nature of the network is determined by the interplay of $L, \xi$ and a third length scale, the finite-size cutoff length $r_{\max } \sim A L^{1 /(\gamma-1)}$ [248]. In addition, this model is an example that power law degree distributions do not necessarily imply the small-world behavior. In fact, in some range of the parameters the model shows fractal scaling as in Ref. [233]. The introduction in the model of an additional parameter that controls the clustering coefficient has been considered in Ref. [250].

In Ref. [249], Warren et al. have studied the properties of a scale-free network $\left(P(k) \sim k^{-\gamma}\right)$ whose nodes are embedded in a 2D lattice and are connected to the $k$ nearest neighbors on the lattice. The model has a non-zero threshold percolation probability $p_{\mathrm{c}}$ for $\gamma>2$, differently from what found by Pastor-Satorras and Vespignani in BA scale-free networks [251,252] (See Section 4.1). Moreover, for $\gamma>2$, the addition of even a sizeable number of smallworld links does not result in $p_{\mathrm{c}}=0$. Due to mapping of the SIR model onto percolation discussed in Section 4.1, such results have important consequences in epidemic propagation and suggest that random immunization in presence of geographical clustering might be more successful in controlling human epidemics than previously believed.

In Ref. [253], Herrmann et al. have studied the inverse problem of how the degree distribution depends on the spatial distribution of the nodes. Different distributions of points on a continuous manifold are considered and the network is constructed by joining two nodes whenever their distance is less than a certain cutoff. The fact that the nodes live in a continuous manifold, rather than a lattice, is important in generating scale-free topologies, since the number of neighbors that a node can have within a certain distance is not limited. The authors have derived a general expression for $P(k)$ as a functional of the distribution of the nodes. For regular spatial densities, the corresponding network has a distribution $P(k)$ decreasing faster than exponential. Networks originating from a uniform distribution of the nodes have been studied previously in Ref. [254], where it was shown that while the connectivity distribution takes the same Poisson form as in ER random graphs, other important features, as the clustering coefficient, are radically different from the ER case; for the formation of giant clusters in such networks see Refs. [254,255]. On the other hand, scale-free networks are obtained when the high powers of the spatial distribution of nodes diverge [253]. A particular important role in the model is played by the case $P(k) \sim k^{-1}$, which appears also to be relevant in real networks of biological origin such as the ones constructed from gene expression data [256-260].

Models that account for the finite capacity in establishing links (that is an important characteristic of many real networks, not only of those embedded in the Euclidean space) have been proposed in Refs. [49,83,261]. Amaral et al. have considered a model of a growing network that evolves according to the usual BA algorithm with linear preferential attachment, but when a vertex reaches a certain critical number of connections $k_{\mathrm{c}}$, its capacity to increase further the degree is inhibited. Numerical simulations show that the resulting $P(k)$ is a power-law with a finite cutoff at $k_{\mathrm{c}}$ [83]. Other mechanisms, connected to the age of the nodes [83], to the nodes' ability in processing information about only a subset of all the nodes the network [261], and to geo-political constraints [49], have been also investigated.

\subsubsection{Modelling real geographical networks}

The Internet is an example of a network with a precise geographical structure which affects the spatial distribution of the information traffic [201]. The most famous of the Internet generators used for the simulation and testing of new technologies [8] is that proposed by Waxman in Ref. [262]. The model is based on two simple ideas: the routers have 

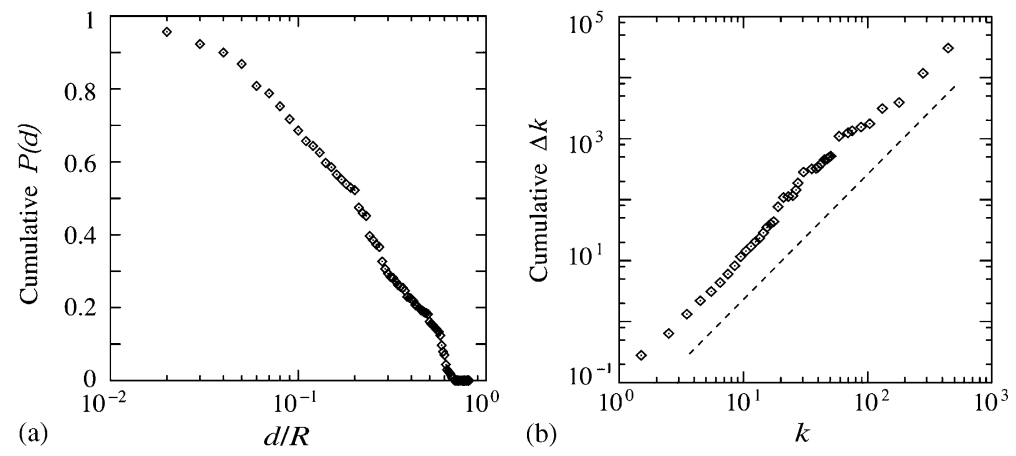

Fig. 2.12. (a) The cumulative length distribution, defined as $\int_{d}^{R} P(x) \mathrm{d} x$, with $d$ being the Euclidean distance between two Internet routers and $R=6378 \mathrm{~km}$ the radius of the Earth, is plotted as a function of the dimensionless variable $d / R$. (b) The cumulative change $\Delta k$ in the connectivity of nodes with $k$ links. The dotted line has slope 2 and indicates that $\Delta k$ is linear in $k$. Figure taken from Ref. [230]. Courtesy of A.L. Barabási.

a random distribution in space and the probability of connecting two routers $i$ and $j$ decays with their distance $\ell_{i j}$ as $P\left(\ell_{i j}\right)=\beta \exp \left(-\ell_{i j} / \alpha D\right)$, where $D$ is the maximum distance between any two nodes, $\alpha>0$ is a parameter used to tune the ratio of short to long distance edges, and $\beta \leqslant 1$ controls the average degree of the network.

In Ref. [230] Yook et al. have proposed to model the Internet as an evolving network, rather than a static one, as done in the classical approaches [8]. In this model, the physical layout of the nodes is a fractal set, as observed empirically (see Fig. 6.8), and the addition of new edges is governed by two competing mechanisms, preferential attachment and geographical distance. The square of linear size $L$ is divided into small squares of size $l \times l$, each square being assigned a population density $\rho(x, y)$ with a fractal dimension $D_{f}$. At each time step a new node $j$ is placed on the map, its position being determined probabilistically, such that the likelihood of placing a node at $(x, y)$ is linearly proportional to $\rho(x, y)$. The new node connects with $m$ links to nodes that are already present in the system. The probability that the new node $j$ links to a node $i$ with $k_{i}$ links and at distance $\ell_{i j}$ is a generalization of the form considered in formula (2.41), namely:

$$
\prod_{j \rightarrow i} \sim k_{i}^{\sigma} \ell_{i j}^{-\alpha}
$$

with the exponents $\sigma$ and $\alpha$ governing respectively preferential attachment and cost of node-node distances. Increasing $\sigma$ will favor linking to nodes with higher degree, whereas a higher value of $\alpha$ will discourage long links. It is important to notice that the probability $P(d)$ of finding two routers at distance $d$ is modelled as a power law instead of the exponential function $P(d) \sim \exp \left(-d / d_{0}\right)$ commonly used in the Waxman model and in most of its generalizations.

The parameters $L, l$ and $m$ are non-universal, as their value can be changed without affecting the network's largescale topology. Conversely, $\sigma, \alpha$, and $D_{f}$ are universal exponents, as their values uniquely parameterize families of Internet models generating different large-scale topologies. Numerical simulations in which the values of the three exponents are changed separately, have allowed to investigate the effect of the different mechanisms on the Internet's large-scale topology. Quite interestingly, it appears that the topological properties observed empirically in the Internet can be obtained only for a particular set of the three exponents, namely $D_{f}=1.5, \sigma=1, \alpha=1$, as confirmed by the plots in Fig. 2.10(a) and in Fig. 2.12. In particular, a linearly decaying cumulative distribution in a log-linear scale, as shown in Fig. 2.12(a), indicates that the probability of connecting two nodes in the Internet is inversely proportional to the distance $(\alpha=1)$. On the other hand, Fig. 2.12(b) offers a direct proof of a linear preferential attachment $(\sigma=1)$.

Moreover, most of the generators currently used to model the Internet produce networks belonging to topological classes different from that of the real Internet. In fact, each Internet generator corresponds to a point in the threedimensional phase space $\left(D_{f}, \sigma, \alpha\right)$, and it can be proved that any deviation from the point $(1.5,1,1)$ representing the real Internet, alters the topological properties of the model defined as in Eq. (2.42).

A model with similar ingredients, although casted in terms of optimization processes [263], is the heuristically optimized trade-off (HOT) model, proposed by Fabrikant et al. [264]. In the HOT model, the Internet is described as 
an evolving tree in which, at each time step, a new node $j$ is randomly placed in a square of linear dimension $L$, and linked to one of the previous nodes. On one hand, the objective is to minimize the Euclidean distance between these two nodes. On the other hand, a newly arrived node would plausibly connect to a node in the tree that is centrally located. The former objective captures the "last mile" costs, the latter the operation costs due to communication delays. Consequently, in the HOT model the new node $j$ is connected to the node $i$ that minimizes the weighted sum of the two objectives:

$$
i: \min _{i<j}\left[\alpha \ell_{i j}+C_{i}\right],
$$

where $\alpha$ is a parameter gauging the relative importance of the two objectives, $\ell_{i j}$ is the Euclidean distance and $C_{i}$ is a measure of the centrality of node $i$ such as: (a) the average number of hops from other nodes (closeness), (b) the maximum number of hops from another node, (c) the number of hops from a fixed center of the tree [263]. The behavior of the model depends crucially on the value of $\alpha$. In the case in which the measure of centrality (c) is adopted, the model can be treated by means of combinatorial-geometric arguments, and the following results hold. If $\alpha$ is less than a given constant $\alpha_{\mathrm{c}}$, then Euclidean distances are not important, and the resulting network is a star. If $\alpha$ grows at least as fast as $\sqrt{N}$, where $N$ is the final number of nodes, the resulting graph is a dynamic version of the Euclidean minimum spanning tree, in which high degrees do occur, but with exponentially vanishing probability. When $\alpha$ is larger than $\alpha_{c}$ and it grows slower than $\sqrt{N}$, then the degrees obey a power law. In the case $\alpha=\mathrm{O}\left(N^{1 / 3}\right)$ one obtains a lower bound of $1 / 6$ for $\alpha_{c}$, while the numerical simulations suggest that the true value is around $0.6-0.9$.

In Refs. [49], Guimerà et al. have studied the structure of the world-wide airport network, finding empirical evidences that both degree and betweenness distributions decay as truncated power laws. To model the real network, the authors have used a variation of the model by Yook at al. [230] and of the model by Barthélemy [245] discussed in Section 2.5.2, that includes to the standard mechanism of growth the addition of links between already existing nodes. Preferential attachment and distance constraints appear to explain the degree and the betweenness distributions but fail to account for the empirical fact that some central airports have small degree. Only a model including geo-political constraints (as the fact that most cities are only allowed to establish connections with other cities within the same country, even though in some case the distance can be larger than that with cities in neighboring countries), allows to generate nodes with high betweenness and small degree, as the ones observed in the real airport network [49].

Gastner and Newman have proposed a comparative study of the spatial structure of three different geographical networks: the US interstate highway network, the Internet and a network of flights operated by a major US airline [265]. The three networks appear to show a preference for short edges over long ones, which is a natural effect of geography. However, the highway network has much shorter edges, lower degrees $(\langle k\rangle=2.86)$, and larger diameter $(=61)$ than the other two. These are all consequences of the planarity of the highway network. In fact, although each of the three networks is, of course, two-dimensional in a geographic sense, since it lives on the two-dimensional surface of the earth, it is possible to show that the road network is, indeed, almost planar [16,265], while the other two networks are not. An effective network dimension $D$ can be determined by using the scaling discussed in Section 2.5.1 [233], namely by plotting $\log N_{i}$ against $\log r$ for some central node $i$, and extracting $D$ as the slope of the initial part of the resulting line $[265,266]$. The slope of the plot is close to 2 for the highways, indicating that this network is essentially two-dimensional. Conversely, for the Internet, the larger slope indicates that the network has higher dimension, or perhaps no well-defined dimension at all (similar results have been obtained for the airline network).

A simple one-parameter model explaining the basic features of geographic networks, including their dimension, can be constructed in terms of competing preferences for either short Euclidean distances between nodes or short graph distances, by assigning to each edge an effective length [265]:

$$
\text { effective length of edge }(i, j)=\lambda \sqrt{N} \ell_{i j}+(1-\lambda) \text {. }
$$

The parameter $0 \leqslant \lambda \leqslant 1$ determines the user's preference for measuring distance in terms of miles or steps: in a road network most travellers look for routes that are short in terms of miles, while for airline travellers the number of changes is often considered more important.

Given the position of $N$ nodes and the budget for building the network (consisting in the maximum total length of the wirings to be used), simulated annealing methods [267] are used to find the network structure that connects all the nodes, can be built within budget, and minimizes the mean distance between all node pairs calculated by using the effective edge lengths defined as in formula 2.44 [265]. In Ref. [265], four networks were considered, generated 


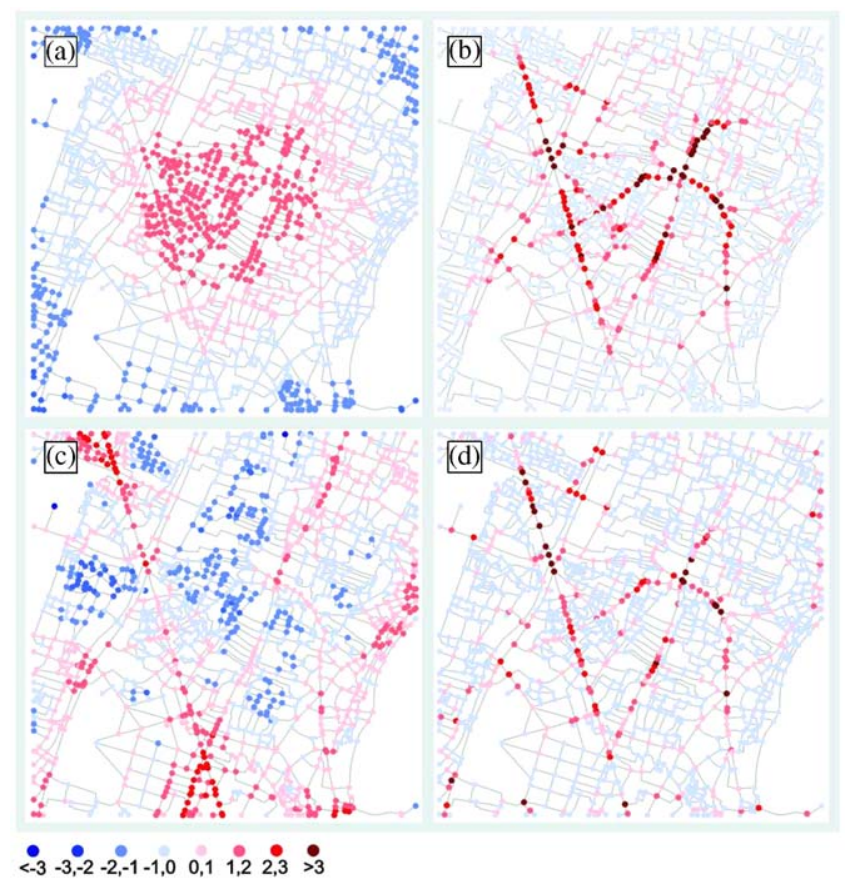

Fig. 2.13. Color coded maps representing the spatial distributions of centrality in Cairo, an example of a largely self-organized city. The four indices of node centrality, (a) closeness; (b) betweenness; (c) straightness and (d) information, are visually compared over the spatial graph. Different colors represent classes of nodes with different values of the centrality index. The classes are defined in terms of multiples of standard deviations from the average, as reported in the color legend. Figure taken from Ref. [47].

by placing $N=50$ vertices at random within a square and by using different values of the parameter $\lambda$. The cases $\lambda=0$ and 1 produce networks strongly reminiscent of airlines and roads respectively. For intermediate values of $\lambda$, the model finds a compromise between hub formation and local links. The model produces networks with a roughly two-dimensional form for large $\lambda$, and a strongly super-quadratic form for small $\lambda$.

In Ref. [268] the same authors have studied a special class of spatial networks, those designed to distribute (or collect) a commodity from (to) a root node that acts as a source (sink) of the commodity. Examples are natural gas pipelines (where the root node is the oil field), city sewer systems (where the root node is the city's sewage treatment plant), and also some transportation systems, as the commuter rail systems in the Boston area [268]. Different aspects of similar networks have been studied in Refs. [36,228]. Such networks possess simultaneously both the benefits of the minimum spanning tree, that is the optimal in terms of total edge length, and the star graph in which every node is connected directly to the root by a single straight line, and have been modelled by a variation of the HOT model [268].

Buhl et al. have studied the efficiency and the robustness of a different kind of tranportation networks, namely those describing galleries in ant nests [33]. The authors have compared the real networks with two extreme cases of planar graphs: minimum spanning trees (graphs with $K=N-1$ links and the minimum total edge length) and minimum weight triangulations (which contain the maximum number of edges with no edge crossings, $K=3 N-6$, while minimizing the total edge length).

Finally, Porta et al. have presented a comprehensive study of centrality distributions named multiple centrality assessment (MCA) over primal geographic [47,269], rather than dual [55,270-274], networks of urban street patterns. Four different measures of centrality, namely closeness, betweenness, straightness and information [37], have been compared over 1-square-mile samples of different world cities. As an example, the spatial distributions for the city of Cairo are reported in Fig. 2.13. The color-coded maps show how different centralities have a good capacity to capture distinct continuous paths in the urban system. In addition, the MCA reveals a certain capacity to characterize different classes of universality for cities (distinguishing, for instance, planned from self-organized cities [47]), and, after an establishment of the correlation between centralities and socio-economic factors, leads to more argumentative indications for strategies of urban planning and design. 


\section{Static and dynamic robustness}

Robustness refers to the ability of a network to avoid malfunctioning when a fraction of its constituents is damaged. This is a topic of obvious practical reasons, as it affects directly the efficiency of any process running on top of the network, and it was one of the first issues to be explored in the literature on complex networks. In this chapter, we review the main results concerning the resilience of networks to both random failures and intentional attacks. The problem can be encountered in two different variants. The first one, referred to as static robustness, is meant as the act of deleting nodes without the need of redistributing any quantity that is being transported by the network. This is the case, for instance, of a social network in which we cut relationships between individuals forming the system. On the other hand, dynamical robustness refers to the case in which the dynamics of redistribution of flows should be taken into account. An example can be found in the Internet where a router occasionally fails to work and its load, in the form of packets, has to be diverted through alternative paths. The two types of robustness are similar in spirit, but while the first can be analytically treated, e.g. by using the tools of statistical physics such as percolation theory, the analytical treatment of the second case is harder and in almost all cases one has to rely on numerical simulations.

\subsection{Static tolerance to errors and attacks}

Static tolerance to errors (or random failures) is understood as the ability of the system to maintain its connectivity properties after the random deletion of a fraction $f$ of its nodes or edges. On the other hand, we refer to an attack when such a deletion process is targeted to a particular class of nodes or edges, for instance to the highly connected nodes. Besides numerical simulations [101,275], a series of analytical approaches [276-280] to study tolerance to errors and attacks in complex networks have been proposed.

The main idea is that the problem can be analytically treated by using percolation theory [281]. A standard percolation process can be, in general, of two types: site or bond. Site percolation on a given graph means that the vertices are empty with a given probability $f$ (or occupied with a probability $1-f$ ), while bond percolation refers to the existence or not of an edge between two arbitrarily chosen nodes. Once the random deletion (or placement) of nodes or edges done, several quantities allow the characterization of the network properties. One usually looks at the existence and size of the giant component as a function of $f$, and at the average size and fluctuations in the size of finite components. In such a way one defines a critical probability $f_{\mathrm{c}}$ below which the network percolates, and a set of critical exponents characterizing the phase transition. The study of random failures in complex networks can be exactly mapped into a standard percolation problem. This is not the case anymore when dealing with attacks, because the removed nodes (links) are not chosen at random. However, as we will see in Section 3.1.4, the same formalism used for errors can be extended with few modifications.

The topic has already been thoroughly revised by other authors as Newman [4] and Cohen et al. [6]. For such a reason, here we shall briefly review the results for uncorrelated networks and concentrate, instead, on correlated networks.

\subsubsection{Numerical results}

The first numerical studies on the robustness of real networks are those reported in Refs. [101,275]. Albert et al. have studied how the properties of the Internet and of a sample of the World Wide Web change when a fraction $f$ of the nodes are removed [275]. The nodes are chosen at random to simulate errors, or in decreasing order of their degree $k$ to simulate attacks.

Both in the Internet and in the World Wide Web the giant component persists for high rates of random node removal while, if the nodes are removed in the attack mode, the size of the fragments that break off increases rapidly. This behavior is in contrast with that observed for random graphs, and consistent with that of scale-free graphs. In a random graph with $N=10,000$ nodes and $\langle k\rangle=4, S$ displays for both errors and attacks a threshold-like behavior such that $S \simeq 0$ for $f>f_{\mathrm{c}} \simeq 0.28$ (panel a). The response of a scale-free network of the same size and $\langle k\rangle$ to attacks and failures is rather different (panel b). For random failures the size of the largest component decreases slowly and no threshold is observed. On the other hand, the response to attacks of the scale-free network is similar to the response to attacks and failures of the random graph network, with a critical value $f_{\mathrm{c}} \simeq 0.18$ smaller than the value observed for the random graph.

Broder et al. have studied a larger data set of the WWW obtaining similar results (although interpreted in an apparently opposite way) [101]. 

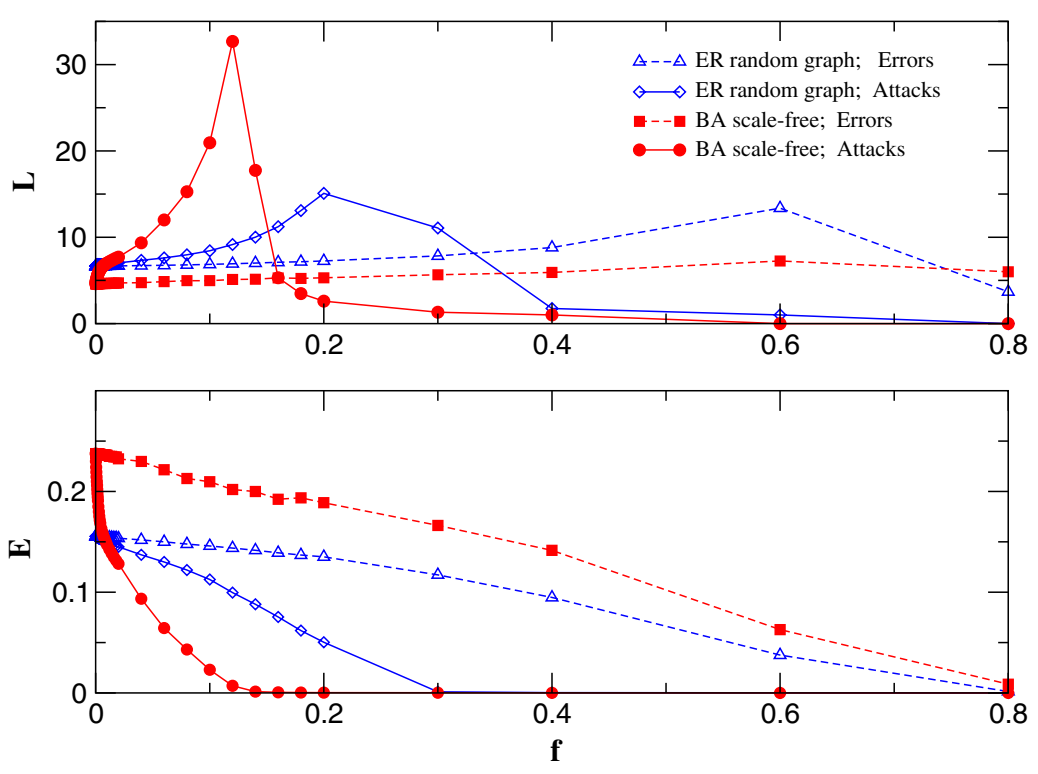

Fig. 3.1. Network robustness under random failures and attacks on nodes. Average shortest path length $L$ and global efficiency $E$ are reported as a function of the fraction of removed nodes $f$. An ER random graph and a BA scale-free network, both with $N=5000$ and $K=10,000$ are considered. Reprinted from Ref. [282], (C) 2003, with permission from Elsevier.

Crucitti et al. have used the concept of network efficiency at both global and local scales to study the effects of errors and attacks on BA and on KE structured scale-free networks [282,283]. Global and local efficiency are defined respectively as in formulas (2.6) and (2.11) and allow an alternative characterization of networks with clustering and small-world property. The numerical results indicate that both the global and the local efficiency of scale-free networks are unaffected by the removal of some (up to $2 \%$ ) of the nodes chosen at random. On the other hand, at variance with random graphs, global and local efficiencies rapidly decrease when the nodes removed are those with the higher connectivity. In Fig. 3.1 we report the characteristic path length $L$ and the global efficiency $E$ for a ER random graph and a BA scale-free network when a large number of nodes (up to $80 \%$ of the nodes of the network) are removed. On such a scale the difference between scale-free networks and random graphs is less pronounced than in the smaller scale $(f<0.02)$. Moreover, when the network becomes unconnected, $E$ is a better quantity than $L$ to describe the system.

Different approaches to study the vulnerability of a network have been proposed by Goh et al. [43], Kim et al. [284], Motter et al. [285], Holme [286,287], and Latora and Marchiori [34].

\subsubsection{Resilience in uncorrelated networks}

To support the numerical results with analytical calculations we follow the argument by Cohen et al. [277,278]. We consider a random graph with $N$ vertices, average connectivity $\langle k\rangle$ and arbitrary degree distribution $P(k)$. We first analyze under which condition the original graph has a giant component. Then, we delete a fraction $f$ of the vertices and we repeat the same analysis on the damaged graph. The crucial point is to notice that a randomly chosen vertex has degree $k$ with probability $P(k)$, while the degree distribution of the nearest neighbor of a randomly chosen vertex is different from $P(k)$. In fact, if we choose at random an edge and randomly take an end vertex following the edge, the degree distribution of that vertex is given by

$$
q_{k}=\frac{k P(k)}{\langle k\rangle} .
$$

This is because we have $k N_{k}$ (where $N_{k}$ is the number of nodes with $k$ edges) edges attached to vertices of degree $k$ out of a total of $\sum_{k} k N_{k}$ edges. Another important point is that cycles can be neglected and the local structure of large sparse uncorrelated networks is tree-like (recall that the clustering coefficient vanishes in generalized random graphs as $N$ goes to infinity). Then, in order for a giant component to exist, a node $i$ connected to a node $j$ in the giant component 
(spanning cluster) should be connected to at least one other node. This condition translates into the statement that if the average number of links of the second nearest neighbors $\left(z_{2}\right)$ of a randomly chosen vertex is greater that the average number of its nearest neighbors $(\langle k\rangle)$, then the network is not fragmented, i.e., it percolates. The quantity $z_{2}$ can be obtained as

$$
z_{2}=\langle k\rangle\left(\sum_{k} k \frac{k P(k)}{\langle k\rangle}-1\right)=\langle k\rangle\left(\frac{\left\langle k^{2}\right\rangle-\langle k\rangle}{\langle k\rangle}\right)=\left\langle k^{2}\right\rangle-\langle k\rangle,
$$

where

$$
\left(\sum_{k} k \frac{k P(k)}{\langle k\rangle}-1\right)
$$

is the average number of connections of any end vertex of any edge, other than the edge from which we come. At criticality $z_{2} /\langle k\rangle=1$, and thus from Eq. (3.2) it immediately follows that

$$
\frac{\left\langle k^{2}\right\rangle}{\langle k\rangle}=2 \text {. }
$$

In terms of the degree distribution $P(k)$, the condition to have a giant component can be rewritten as

$$
\left\langle k^{2}\right\rangle-2\langle k\rangle=\sum_{k} k(k-2) P(k)>0,
$$

which is the Molloy and Reed criterion [122,123] already mentioned in Section 2.3.2 and in Section 2.3.4. This means that a giant component is always present in a generalized random graph if the second moment of the degree distribution diverges. For instance, Eq. (3.4) tells us that a giant component exists in scale-free networks with exponents in the range $2<\gamma<3$, as those found in real networks.

Up to now, we have analyzed the conditions for the existence of the giant component in a network that has not yet been damaged. We restrict our discussion to the case of site percolation in which a fraction $f$ of the nodes is randomly deleted. In a similar manner one can study also the problem of bond percolation. If the damage process does not destroy the fat-tail of the degree distribution of the new network $G^{\prime}$ (so that its second moment still diverges), then a giant component will persist to show up. After the random deletion of a fraction $f$ of nodes, a vertex with former connectivity $k$ will be now attached to a number $k^{\prime}$ of other (existing) vertices. This number is distributed binomially, so that the total probability that a randomly chosen vertex is connected to $k^{\prime}$ other vertices is given by

$$
P\left(k^{\prime}\right)=\sum_{k=k^{\prime}}^{\infty} P(k)\left(\begin{array}{c}
k \\
k^{\prime}
\end{array}\right)(1-f)^{k} f^{k-k^{\prime}} .
$$

From Eq. (3.5) Cohen et al. [277] obtained the new criterion for criticality by substitution of the new $\left\langle k^{\prime 2}\right\rangle$ and $\left\langle k^{\prime}\right\rangle$ in Eq. (3.3), yielding

$$
1-f_{\mathrm{c}}=\frac{1}{\left(\left\langle k^{2}\right\rangle /\langle k\rangle\right)-1},
$$

which, as before, states that if the second moment of the original network is diverging in the thermodynamic limit, the network does not have a percolation threshold, or, strictly speaking, the percolation threshold is zero.

The result gathered in Eq. (3.6) can be obtained in several other ways. Here we would like to mention two more rigorous approaches that can be adopted. The first is a byproduct of techniques used in statistical mechanics of disordered systems. It was first introduced by Vázquez et al. in the study of ferromagnetic ordering in graphs with arbitrary degree distribution [288,289]. The formalism was later applied to the study of covering problems in networks with correlations [288]. The second is due to Newman et al. [124,276] and is based on the generating function formalism. In the spirit of this approach, let us assume that after the random removal of vertices in the original network, a vertex is still present in the resulting graph $G^{\prime}$ with probability $1-f$, and that with probability $x$ a randomly chosen edge in the original 
network leads to a vertex in $G^{\prime}$ that belongs to a finite connected component. Then it can be shown that $x$ satisfies the self-consistent equation:

$$
x=f+(1-f) \sum_{k=0}^{\infty} \frac{k P(k)}{\langle k\rangle} x^{k-1},
$$

where the first term accounts for the possibility that the end vertex has been removed. The terms in the sum takes into account the fact that the end vertices are distributed according to $k P(k) /\langle k\rangle$ and each of them is present with probability $x$. Eq. (3.7) has a non-trivial solution $(x<1)$ if the giant component exists. Then, by linearizing it, one can find the percolation threshold $f_{\mathrm{c}}$, yielding again formula (3.6).

\subsubsection{Resilience in correlated networks}

We now assume that correlations in the degree of adjacent vertices are present. We closely follow the arguments of Refs. [280,288]. In this case, the degree distribution is not enough, and one has to work with the conditional probability that a vertex of degree $k^{\prime}$ is reached following any edge coming from a vertex of degree $k$. Denoting by $E_{k^{\prime} k}$ the symmetric matrix that measures the number of edges between vertices of degree $k^{\prime}$ and $k$ and two times the number of self-connections when $k=k^{\prime}$, we have that

$$
P\left(k^{\prime} \mid k\right)=\frac{E_{k^{\prime} k}}{\sum_{k^{\prime}} E_{k^{\prime} k}}=\frac{E_{k^{\prime} k}}{k N_{k}},
$$

where $N_{k}$ is the number of vertices with degree $k$. Furthermore, the probability that a randomly chosen edge connects two vertices of degrees $k^{\prime}, k$ is given by $\left(2-\delta_{k^{\prime}, k}\right) e_{k^{\prime} k}$, where $e_{k^{\prime}, k}$ is the joint probability defined as

$$
e_{k^{\prime}, k}=\frac{E_{k^{\prime} k}}{\sum_{k} \sum_{k^{\prime}} E_{k^{\prime} k}}=\frac{E_{k^{\prime} k}}{\langle k\rangle N} .
$$

We thus rewrite $P\left(k^{\prime} \mid k\right)$ as

$$
P\left(k^{\prime} \mid k\right)=e_{k^{\prime} k} / q_{k} .
$$

Consistency with the degree distribution requires $\sum_{k=0}^{\infty} e_{k^{\prime} k}=q_{k^{\prime}}$, being $e_{k^{\prime} k}$ symmetric. For uncorrelated networks $e_{k^{\prime} k}=q_{k^{\prime}} q_{k}$ factorizes, giving $P\left(k^{\prime} \mid k\right)=q_{k^{\prime}}$, that is independent of $k$. Additionally notice that, for instance, the strongest positive correlations are obtained for $e_{k^{\prime} k}=q_{k^{\prime}} \delta_{k^{\prime} k}$, i.e. when only vertices of equal degrees are connected.

The problem of percolation is equivalent to the Ising model at zero temperature and zero magnetic field, where the size of the giant component corresponds to the magnetization [289,290]. In fact, as pointed out by Dorogovtsev and Mendes [7], it is a particular case of the general $n$-state Potts model. This analogy can be exploited to study the percolating properties of random networks with arbitrary degree correlations. Alternatively, one can follow the generating function formalism to obtain the fraction of nodes $S$ in the giant component and the average probability $u_{k}$ that an edge connected to a vertex of degree $k$ leads to another vertex that does not belong to the giant component:

$$
\begin{aligned}
& S=1-\sum_{k} P(k)\left(u_{k}\right)^{k}, \\
& u_{k}=\sum_{k^{\prime}} P\left(k^{\prime} \mid k\right)\left(u_{k^{\prime}}\right)^{k^{\prime}-1} .
\end{aligned}
$$

We can now discuss the conditions for the existence of a giant component after the random removal of either vertices or edges. We shall derive some general expressions that allow the computation of the transition thresholds or their bounds for both site and bond percolation on power law correlated graphs.

We first consider the case of site percolation, in which a fraction $f$ of the nodes is removed from the graph and the new giant component is computed. Because the node removal is independent of the node degree, the original degree distribution and correlations can be respectively replaced by (i) the probability that a node selected at random has degree $k$ and it has not been removed, and (ii) the probability that if we select a node at random and follow one of its edges we end in a node with degree $k^{\prime}$ that has not been removed:

$$
P(k) \rightarrow(1-f) P(k), \quad P\left(k^{\prime} \mid k\right) \rightarrow(1-f) P\left(k^{\prime} \mid k\right) .
$$


Substitution of Eqs. (3.13) in Eqs. (3.11) and (3.12) gives

$$
\begin{aligned}
& S=1-f-(1-f) \sum_{k} P(k)\left(u_{k}\right)^{k}, \\
& u_{k}=f+(1-f) \sum_{k^{\prime}} P\left(k^{\prime} \mid k\right)\left(u_{k^{\prime}}\right)^{k^{\prime}-1},
\end{aligned}
$$

where the term $-f(f)$ in Eq. (3.14) (Eq. (3.15)) gives the probability of hitting a node that has been removed. Note that Eq. (3.15) reduces to Eq. (3.7) for uncorrelated networks. The solution $u_{k}=1$ and $S=0$ to these equations is valid whenever the equation for $u_{k}$ is stable under successive approximations. In other words, if we start with $u_{k}(n)=1-\rho_{k}(n)$ and compute the successive approximations $\rho_{k}(n+1), n=1, \ldots, \infty$, we should obtain that $\rho_{k}(n) \rightarrow 0$ in the limit $n \rightarrow \infty$. For $\rho_{k}(n) \ll 1$ the last equation is approximated by the (linear) map:

$$
\rho_{k}(n+1)=\sum_{k^{\prime}} L_{k k^{\prime}} \rho_{k^{\prime}}(n)
$$

with

$$
L_{k k^{\prime}}=(1-f) C_{k k^{\prime}}, \quad C_{k k^{\prime}}=\left(k^{\prime}-1\right) P\left(k^{\prime} \mid k\right) .
$$

We have come to the criterion we were looking for: the stability of the solution $u_{k}=1$ is related to the largest eigenvalue of $L_{k k^{\prime}}$. Therefore, if it is smaller (larger) than 1 the solution is stable (unstable). Since $L_{k k^{\prime}}$ is linear in $f$, the stability condition reduces to:

$$
f>f_{\mathrm{c}}, \quad\left(1-f_{\mathrm{c}}\right) \Lambda_{\max }=1,
$$

where $\Lambda_{\max }$ is the largest eigenvalue of $C_{k k^{\prime}}$ provided that $\Lambda_{\max }>1$. Note that if $\Lambda_{\max }<1$, the graph does not have a giant component even for $f=0$. Moreover, since $C_{k k^{\prime}}$ is a positive matrix, then $\Lambda_{\max }$ is bounded by $\min _{k} \sum_{k^{\prime}} C_{k k^{\prime}}$ and $\max _{k} \sum_{k^{\prime}} C_{k k^{\prime}}$, which gives:

$$
\min _{k} k_{n n}(k) \leqslant 1+\Lambda_{\max } \leqslant \max _{k} k_{n n}(k),
$$

where

$$
k_{n n}(k)=\sum_{k^{\prime}} P\left(k^{\prime} \mid k\right) k^{\prime}
$$

is the average degree among the neighbors of a node with degree $k$ (see Section 2.1.1). Eq. (3.19) can be used to determine, based on a simple topological measure, whether or not a given graph is robust under vertex removal.

We now consider bond percolation. In such a case, a fraction $f$ of the edges is removed from the graph and the new giant component is computed. Since the edges are chosen at random, this is equivalent to keep the original degree distribution and replace the degree correlations by the probability that if we select a node at random and follow one of its edges we end in a node with degree $k^{\prime}$ that has not been removed, i.e.

$$
P(k) \rightarrow P(k), \quad P\left(k^{\prime} \mid k\right) \rightarrow(1-f) P\left(k^{\prime} \mid k\right) .
$$

As before, substitution of Eq. (3.21) in Eqs. (3.11) and (3.12) yields

$$
\begin{aligned}
& S=1-\sum_{k} P(k)\left(u_{k}\right)^{k}, \\
& u_{k}=f+(1-f) \sum_{k^{\prime}} P\left(k^{\prime} \mid k\right)\left(u_{k^{\prime}}\right)^{k^{\prime}-1} .
\end{aligned}
$$

Note that the only difference between the site and bond percolation problems (see Eqs. (3.14) and (3.15)) is found in the equation for the giant component, while that for $u_{k}$ is identical. Hence, Eqs. (3.18) and (3.19) are also valid for the bond percolation problem. This is not surprising, and in fact reproduces the behavior already pointed out for 
uncorrelated networks. Indeed, for random graphs we have $P\left(k^{\prime}, k\right)=q_{k^{\prime}}$. In this case, the lower and upper bounds in Eq. (3.19) are equal giving for the largest eigenvalue:

$$
\Lambda_{\max }^{\text {unco }}=\frac{\left\langle k^{2}\right\rangle}{\langle k\rangle}-1
$$

Alternatively one can compute $\Lambda$ directly from the eigenvalue problem of $C_{k k^{\prime}}$. By substitution in Eq. (3.18) one gets Eq. (3.6) discussed in Section 3.1.2. Namely, if the second moment $\left\langle k^{2}\right\rangle$ diverges, the threshold equals 1, i.e. the network is robust under random vertex or edge removal.

Additionally, consider the case in which the degree correlations can be decomposed into two components:

$$
P\left(k^{\prime} \mid k\right)=\alpha q_{k^{\prime}}+(1-\alpha) \delta P\left(k^{\prime} \mid k\right),
$$

with $0<\alpha<1$ and $\delta P\left(k^{\prime} \mid k\right)>0$ for all $\left(k, k^{\prime}\right)$. By tuning the parameter $\alpha$, one interpolates between the uncorrelated graphs $(\alpha=1)$ and a graph with arbitrary degree correlations given by $\delta P\left(k^{\prime} \mid k\right)$. In this case, from Eq. (3.19), $\Lambda_{\max } \geqslant \alpha \Lambda_{\max }^{\text {unco }}$ is verified and, therefore, if the network is robust for the uncorrelated case it will also be robust for any $\alpha>0$. The conclusion that follows immediately is that any graph with a finite amount of random mixing of edges for which the second moment diverges does not have a percolation threshold.

An interesting question is whether or not the divergence of the second moment is a necessary or sufficient condition for the absence of the threshold in correlated graphs. The answer to these issues can be obtained by analyzing networks with different correlations. Let us make the case of a disassortative graph. Consider the graph built up according to the following rules. A vertex with degree $k>1$ is attached with probability $g_{k}$ to a vertex chosen at random among all vertices with degree $k^{\prime}>1$, otherwise it is connected to a vertex with $k^{\prime}=1$ also chosen at random, i.e.

$$
P\left(k^{\prime} \mid k\right)=\frac{\left(1-g_{k^{\prime}}\right) k^{\prime} P\left(k^{\prime}\right)}{\sum_{s}\left(1-g_{s}\right) s P(s)} \Theta\left(k^{\prime}-1\right) \delta_{k, 1}+\left(1-g_{k}\right) \delta_{k^{\prime}, 1} \Theta(k-1)+g_{k} \frac{g_{k^{\prime}} k^{\prime} P\left(k^{\prime}\right)}{\sum_{s} g_{s} P(s)} \Theta\left(k^{\prime}-1\right) \Theta(k-1),
$$

where $\Theta(x)$ is the unitary step function $(\Theta(x)=0$ for $x \leqslant 0$ and $\Theta(x)=1$ for $x>0)$. Besides, the fraction of nodes with degree 1 is obtained self-consistently from the condition $p_{1}=\sum_{k>1}\left(1-g_{k}\right) k P(k)$. From $P\left(k^{\prime} \mid k\right)$ it is straightforward to obtain the average degree of the neighbors of a node with $k>1$ :

$$
k_{n n}=1+g_{k}\left(\frac{\sum_{k^{\prime}>1} g_{k^{\prime}} k^{\prime 2} P\left(k^{\prime}\right)}{\sum_{s} g_{s} s P(s)}-1\right) .
$$

From Eq. (3.27) it is clear that a graph constructed in this way is disassortative for any monotonic decreasing function $g_{k}$. Moreover, we can compute exactly the largest eigenvalue of $C_{k k^{\prime}}=\left(k^{\prime}-1\right) P\left(k^{\prime} \mid k\right)$, resulting:

$$
\Lambda_{\max }=\frac{\sum_{k} g_{k}^{2}(k-1) k P(k)}{\sum_{s} g_{s} P(s)} .
$$

Hence, the conditions for the existence of a giant component $\left(\Lambda_{\max }>1\right)$ or resilience to damage $\left(\Lambda_{\max }=\infty\right)$ are modulated by $g_{k}$ and, therefore, the disassortative correlations given by $g_{k}$ have a great impact on the percolation properties. For instance, let us assume that

$$
g_{k}=k^{-\alpha} \quad \text { and } \quad P(k)=c k^{-\gamma},
$$

with $\gamma<3\left(\left\langle k^{2}\right\rangle=\infty\right)$. From Eq. (3.27) it follows that:

$$
k_{n n}-1 \sim k^{-\alpha}
$$

so that the graph gets more and more disassortative as $\alpha$ is increased. Moreover, $\Lambda_{\max }$ diverges for $\alpha<\alpha_{\mathrm{c}}=(3-\gamma) / 2$ and it is finite otherwise. Thus, the graph is robust for small values of $\alpha$, but for $\alpha>\alpha_{\mathrm{c}}$ it becomes fragile. It is worth noticing that the value of $\alpha$ above which the giant component disappears $\left(\Lambda_{\max }<1\right)$ is larger than $\alpha_{c}$. Besides, for large degrees, the degree distribution of the vertices in the giant component is still a power law, but it decays slower than that of the whole graph. Thus, disassortative correlations compete against the formation of the giant component, and the 
divergence of $\left\langle k^{2}\right\rangle$ is not a sufficient condition to get a robust graph with $f_{\mathrm{c}}=1$. The situation can be worse since the giant component may not even exist. An extreme example of this case is given by the graph made up of a collection of stars interconnected by nodes with degree $m$.

For a power law distributed graph $P(k) \sim k^{-\gamma}$ it can be shown that the fraction of nodes in the largest cluster scales as $N^{1 /(\gamma-1)-1}$ and, therefore, goes to zero when $N \rightarrow \infty$. This results holds for any degree distribution, independently on whether the second moment is finite or not. Hence, we again have that the divergence of $\left\langle k^{2}\right\rangle$ is not a sufficient condition for a graph to percolate. This seems to be a trivial result, but it simply means that there are networks for which the second moment can be formally defined although they do not show up a giant component even for the undamaged case. This is similar to the condition derived in [276,278].

Thus, in general, disassortative correlations make the giant component smaller as compared with that of a random graph with the same degree distribution, and it is not excluded that their presence can tune it to zero. Altogether, the conclusion is that disassortative correlations make it more difficult the formation of the giant component and can make a graph fragile even with a divergent second moment.

\subsubsection{Tolerance to attacks}

We now discuss the problem of tolerance to attacks in uncorrelated networks. In Section 3.1.2 we showed that a giant component exists if the equality $\left\langle k^{2}\right\rangle /\langle k\rangle=2$ is satisfied (see Eq. (3.3)). At the same time, after the random removal of a fraction $f$ of nodes, the new criterion for criticality reduces to Eq. (3.6). Let us now consider that the removal of nodes is targeted to the highly connected nodes, and follow the derivation by Cohen et al. [278]. Such an attack changes the connectivity distribution of the remaining vertices because of the deletion of vertices and, more important, of many links emanating from the removed nodes. The cutoff connectivity, $\kappa$, before the attack could be estimated from

$$
\sum_{k=\kappa}^{\infty} P(k)=\frac{1}{N} \text {. }
$$

Analogously, the cutoff connectivity $\tilde{\kappa}$ after the attack satisfies the relation:

$$
\sum_{k=\tilde{\kappa}}^{\kappa} P(k)=\sum_{k=\tilde{\kappa}}^{\infty} P(k)-\frac{1}{N}=f,
$$

where $f$ is the fraction of removed nodes with the highest degree. In the continuous $k$ limit and for large system sizes $(f \gg 1 / N)$, Eq. (3.32) gives

$$
\tilde{\kappa}=k_{\min } f^{1 /(1-\gamma)}
$$

where $k_{\min }$ is the minimum connectivity of the original distribution $P(k)$. Since the network is random, the probability that a link emanating from a node of degree $k$ that has been removed leads to a node of connectivity $k^{\prime}$ is independent of $k^{\prime}$. Hence, all edges have the same probability to be removed. Therefore, the impact of the attack on the distribution of remaining nodes results in a random removal of edges from the nodes that are left in the network. The difference with respect to random deletion is that the removal of highly connected nodes affects a large number of edges (recall that the hubs control the connectedness of the system), and thus the distribution given by Eq. (3.5) changes as well as the probability of removing a link, which is not anymore given by $f$. In particular, as the cutoff connectivity is now smaller, the probability $\tilde{f}$ that an edge chosen at random points to one of the removed nodes is given by

$$
\tilde{f}=\sum_{k=\tilde{\kappa}}^{\kappa} \frac{k N_{k}}{\sum k N_{k}}=\sum_{k=\tilde{\kappa}}^{\kappa} \frac{k P(k)}{\sum k P(k)}=\sum_{k=\tilde{\kappa}}^{\kappa} \frac{k P(k)}{\langle k\rangle},
$$

being $\langle k\rangle$ the average connectivity before the attack. Using again the continuous $k$ approximation and for large $N$ it is finally obtained:

$$
\tilde{f}=\left(\frac{\tilde{\kappa}}{k_{\min }}\right)^{2-\gamma}=f^{(2-\gamma) /(1-\gamma)},
$$

for $\gamma>2$ [278]. Eq. (3.35) clearly states that very small values of $f$ are enough to destroy a large fraction of edges as $N$ grows. For instance, in the extreme case of $\gamma=2$, it can be shown [278] that Eq. (3.35) reduces to $\tilde{f}=\ln \left(N f / k_{\min }\right)$. 
Additionally, roughly speaking, if nodes are removed with probability $f=0.1$ targeting the highly connected ones, nearly $70 \%$ of the edges are deleted from a network with a exponent $\gamma=2.2$ (as the case of the Internet). Finally, Cohen et al. argued that the same formalism used for the random breakdown problem can be used here, and that the critical point $f_{\mathrm{c}}$ (though not the size of the giant component) is not affected.

Using the approximation described in Section 3.1.2 it can be derived that [278]

$$
\left(\frac{\tilde{\kappa}}{k_{\min }}\right)^{2-\gamma}-2=\frac{2-\gamma}{3-\gamma} k_{\min }\left[\left(\frac{\tilde{\kappa}}{k_{\min }}\right)^{3-\gamma}-1\right],
$$

whose numerical solution allows the computation of $f_{\mathrm{c}}\left(k_{\min }, \gamma\right)$ through Eq. (3.33). Several additional quantities, such as the fraction of the nodes belonging to the spanning cluster, can also be calculated following the generating function techniques developed in [124,276].

In summary, targeted deletion of nodes in uncorrelated scale-free networks are highly effective if compared to random breakdown, as previously anticipated by the numerical simulations by Albert et al. [275]. This is due to the critical high degree of just a few vertices whose removal disrupts the whole network. On the other hand, tolerance to attacks in correlated networks is still a problem entirely to be explored. This is due in part to the lack of generic models that produce networks with arbitrary degree-degree correlations. In fact, just a few works have dealt with the problem of generating such networks [148].

\subsection{Dynamical effects}

In the previous section we have focussed on the static properties of a network, showing the effects caused by the removal of a certain percentage of nodes or links. However, in real systems, there is another important dimension to add to the problem, that refers to modelling the dynamics of flows of the physical quantities of interest over the network. A full characterization of a network cannot be fully accounted for without considering the interplay between structural and dynamical aspects. When it comes to modelling the dynamics, the situation is very complicate, since the components of a network may have different transmission capacities, and the load is often a highly variable quantity both in space and in time. For what concerns the subject of this chapter, fluctuations due to external factors can have much more devastating consequences when the dynamics of flows of physical quantities in the network is properly accounted for. Here we will discuss, in Section 3.2.1, the problem of avalanches of node breakings, and, in Section 3.2.2, the problem of congestion in communication systems. The models we shall present have only a qualitative role as explanatory theory. Nevertheless, they provide indications on the actions that can be performed in order to decrease the undesired effects.

\subsubsection{Modelling cascading failures}

Avalanche of breakdowns over the network is a serious threat when node and links are sensitive to overloading. In a power transmission grid, for instance, each node (power station) deals with a load of power. The removal of nodes, either by random breakdown or intentional attacks, changes the balance of flows and leads to a global redistribution of loads over all the network that can be, in some cases, not tolerated and might trigger a cascade of overload failures. An example is the blackout of 11 US states and two Canadian provinces on the 10th August 1996 [291,292], initially due to the sag of a $1300 \mathrm{MW}$ electrical line in southern Oregon. Probably this is also the reason why on August 14, 2003 an initial disturbance in Ohio [293] triggered the largest blackout in the US's history, in which millions of people remained without electricity for as long as $15 \mathrm{~h}$. These, and similar examples, constituted the motivation for the study of how the extent and the dynamics of the avalanches are dependent on the network structure.

In Ref. [294] the fiber bundle model [295,296] on scale-free networks is studied as a conceptual framework useful to model cascading failures. In this model, the system is subjected to an external pressure (load). The behavior of several quantities indicates that the system exhibits a sort of critical point which depends on both the architecture of the underlying network and the heterogeneity of nodes' capacities. More important, the results point out that, in order to prevent the breakdown of scale-free networks, one has to find an optimal criterion that takes into account two factors: the robustness of the system itself under repeated failures, and the possibility of knowing in advance that the collapse of the system is approaching. 


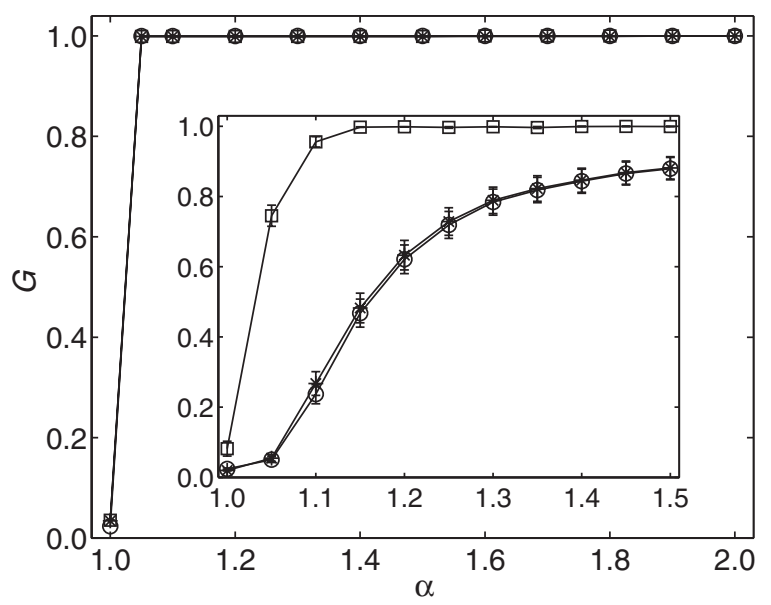

Fig. 3.2. Size of the largest connected component as a function of the tolerance parameter in the model for cascading failures by Motter et al. [298]. Cascades are triggered by the removal of a node chosen at random (squares), or by the removal of the node with largest load (circles) or with the largest degree (asterisks). Homogeneous networks (random graphs with all nodes having $k=3$ links) are considered in the main frame, while heterogeneous networks (scale-free networks with an average degree equal to 3 ) are considered in the inset. Both the networks considered have $N=5000$. Reprinted figure with permission from Ref. [298]. (C) 2002 by the American Physical Society.

The model discussed in Ref. [297] is similar in spirit to that of Ref. [294], but there are several important differences. First of all, the evolution rules are now performed on the links and not on the vertices. Moreover, in one case (Ref. [297]) there are several nucleation points for the avalanches, whereas in [294] there is always one, and thus the breaking process follows a unique branching tree (following the links of the network). The two models have two qualitatively different phase diagrams: the first one resembles a first order phase transition, while the other indicates a second order transition. This allows, for example, to obtain a region in which there is no either free flow nor macroscopic congestion [297]. In this region, the number of simultaneous line casualties follows a power-law, in agreement with measures of packet flow in the Internet.

In Ref. [298] Motter and Lai have introduced a simple model for cascades of overload failures. The model shows how an even small fraction of highly loaded nodes can trigger global cascades in networks with heterogeneous distribution of loads. It is based on the assumption that the relevant quantity is exchanged between every couple of nodes and transmitted along the shortest path connecting them. The load at a node $i$, at time $t$, is assumed to be equal to its betweenness $b_{i}(t)[38,39,42,44]$ (see Section 2.1.2). Each node is characterized by a finite capacity, defined as the maximum load that the node can handle. This implies that, if $C_{i}$ is the capacity of node $i$, then the node operates in a free-flow regime when $b_{i}(t) \leqslant C_{i}$. The capacity is assumed to be proportional to the initial load of the node: $C_{i}=\alpha \cdot b_{i}(t=0), \forall i=1,2, \ldots, N$, with $\alpha \geqslant 1$ being a tolerance parameter of the network. The condition $\alpha \geqslant 1$ guarantees that, at time $t=0$, no node is overloaded and the system is working properly.

The removal of a small fraction of nodes, or even of a single node, simulating the breakdown of an Internet router or of an electrical substation, starts the dynamics of redistribution of flows over the network. In fact, such removals change the shortest paths between nodes and, consequently, the distribution of the loads, creating overloads on some nodes. All the overloaded nodes are removed simultaneously from the network. This leads to a new redistribution of loads and subsequent overloads may occur. The new overloaded nodes are removed and the redistribution process continues until when, at a certain time $t^{\prime}$, all the remaining nodes satisfy the condition $b_{i}\left(t^{\prime}\right) \leqslant C_{i}$. The damage caused by a cascade is measured in terms of the relative size of the giant component $G$ of the final network. Fig. 3.2 shows the results of the model as a function of the tolerance parameter $\alpha$ for an artificially created homogeneous graph and a scale-free topology.

The homogeneous network does not experience cascading failures due either to random breakdown or to intentional attacks for $\alpha$ as small as 0.05 . For the heterogeneous network, for the same value of $\alpha$, cascades triggered by the attack on a key node can reduce the giant component to less than $10 \%$ of the original size, as shown in the inset. In this sense homogeneous networks appear to be more resistant to cascading failures than the heterogeneous ones. 
Since in a heterogeneous network the damage of a single component has, in principle, the potential to trigger a global cascade, a fundamental question regards the possible strategies of defense to prevent the cascade from propagating through the entire network. Very often, the only operation allowed in order to reduce the size of the cascade induced by the initial damage is the further removals of nodes and edges. In fact, other methods of defense, as the rewiring of some edges or the addition of new nodes and edges, would involve extra costs and require longer times than those available. In Ref. [299], Motter has showed that a strategy of defense based on the selective further removal of nodes and edges, right after the initial attack or failure and before the propagation of the cascade can, in fact, constitute an efficient strategy of defense. The main result is that the size of the cascade can be drastically reduced with the intentional removal of nodes having small load and edges having large excess of load [299]. Even though any removal always increases the immediate damage on the network, the resulting $G$ is in this case significantly larger, as compared to the case without defense.

In Refs. [50,300] Crucitti et al. have proposed a variation to the previous model in which, instead of permanently removing the overloaded nodes, the communication through these nodes is degraded, so that eventually the flow of the relevant quantities (information or energy) will avoid them. In this sense, the model can be considered as well as a model for congestion in communication networks. The model assumes that the network is described by a weighted graph. The weight $w_{i j}$ attached to the edge $i-j$ is a number in the range $(0,1]$ and measures the efficiency in the communication along the edge. For instance, in the Internet, the smaller $w_{i j}$ is, the longer it takes to exchange an unitary packet of information along the arc between $i$ and $j$. At time $t=0$, the weights of all the edges are set to be equal to one, meaning that all the transmission lines work perfectly and are equivalent. The removal of a node starts the dynamics of the model: it changes the most efficient paths (the shortest ones) between nodes and consequently the distribution of the loads, creating overloads on some nodes. The overloaded nodes are not removed. Instead, at each time $t>0$, for each overloaded node $i$ the following weights update is adopted:

$$
w_{i j}(t+1)=\left\{\begin{array}{ll}
w_{i j}(0) \cdot \frac{C_{i}}{b_{i}(t)} & \text { if } b_{i}(t)>C_{i} \\
w_{i j}(0) & \text { if } b_{i}(t) \leqslant C_{i}
\end{array} \quad \forall j \in \mathscr{N}_{i}\right.
$$

meaning that the efficiency of all the arcs passing through node $i$ are reduced so that eventually the flow will take alternative paths. The damage caused by a cascade is quantified in terms of the decrease in the network efficiency $E$ [30,31] defined in Section 2.1.2.

In Fig. 3.3 we report the value of the efficiency after the system has relaxed to a stationary state, as a function of the tolerance parameter $\alpha$. ER random graphs and the BA scale-free graph are compared, and two different triggering strategies, random and load-based removals, are considered. Both for the random and for the scale-free network we observe a decrease of the efficiency for small values of the tolerance parameter $\alpha$, and the collapse of the system for values smaller than a critical value $\alpha_{\mathrm{c}}$. ER random graphs appear to be more resistant to cascading failures than BA scale-free graphs, as also found in the model of Ref. [298]. In both cases the collapse transition is always sharper for load-based removals than for random removals.

The heterogeneity of the network plays an important role in the network stability. ER random graphs have an exponential load distribution while BA networks exhibit a power law distribution in the node load [42]. This makes a large difference between random removals and load-based removals in BA scale-free networks. In fact there are few nodes, the ones with extremely high initial load, that are far more likely than the other nodes (the majority of the network nodes) to trigger cascades. Fig. 3.3b shows the existence of a large region in the tolerance parameter $(1.1 \leqslant \alpha \leqslant 1.3)$ where scale-free networks are stable with respect to random removals and are unstable with respect to load-based removals. If, for instance the nodes work with a tolerance of $30 \%$ above the standard load $(\alpha=1.3)$, the network is in general very stable to an initial shock consisting in the breakdown of a node. This means that, in most of the cases, the failure is perfectly tolerated and reabsorbed by the system. However, there is always a finite, though very small, probability that the failure triggers an avalanche mechanism collapsing the whole network.

In Ref. [301] Kinney et al. have applied the model by Crucitti et al. to model cascading failures in the North American power grid. The entire electric grid in North America is really just a big circuit and one of the largest and most complex systems of our time. For economic and political reasons the grid has grown in an extremely interconnected way: different utilities can buy and sell electrical energy to one another over ever-increasing distances to take advantage of differentials in the cost of electric services [302,303]. Unfortunately, the same capabilities that allow power to be transferred over hundreds of miles also enable the propagation of local failures into grid-wide events [304,305]. The database considered contains information about every power plant, major substation, and $115-765 \mathrm{kV}$ power line of 

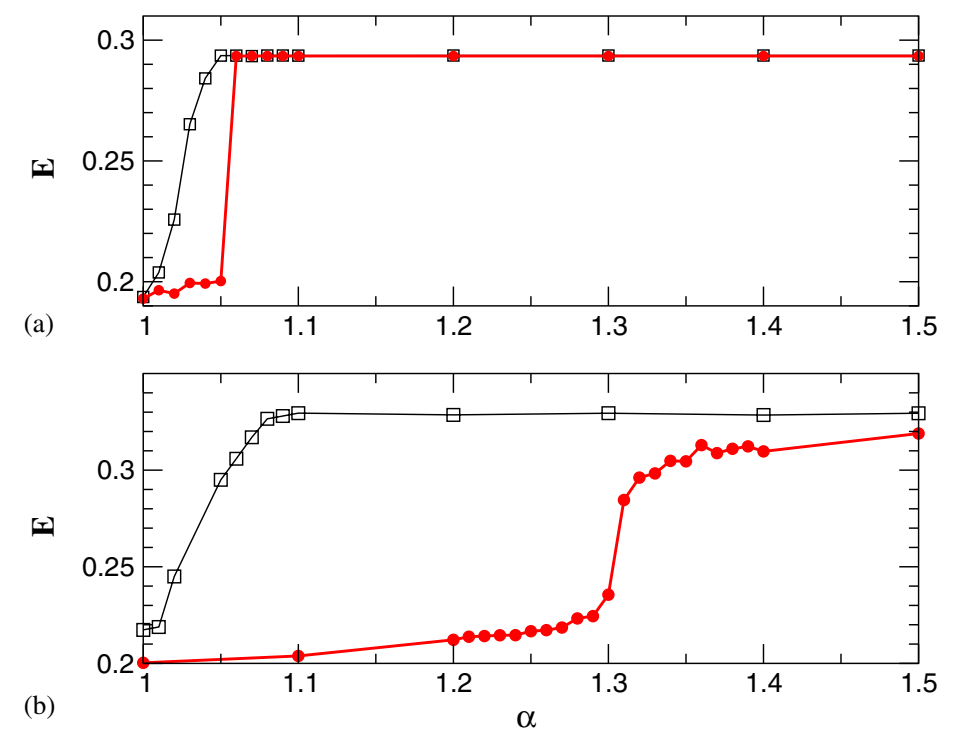

Fig. 3.3. Model for cascading failures by Crucitti et al. [300]. Cascading failure in (a) ER random graphs and (b) BA scale-free networks as triggered by the removal of a node chosen at random (squares), or by the removal of the node with largest load (circles). Both the networks considered have $N=2000$ and $K=10,000$. The final efficiency $E$ of the network is reported as a function of the tolerance parameter $\alpha$. In the case triggered by the removal of a node chosen at random the curve corresponds to an average over 10 triggers. Reprinted figure with permission from Ref. [300]. (C) 2004 by the American Physical Society.
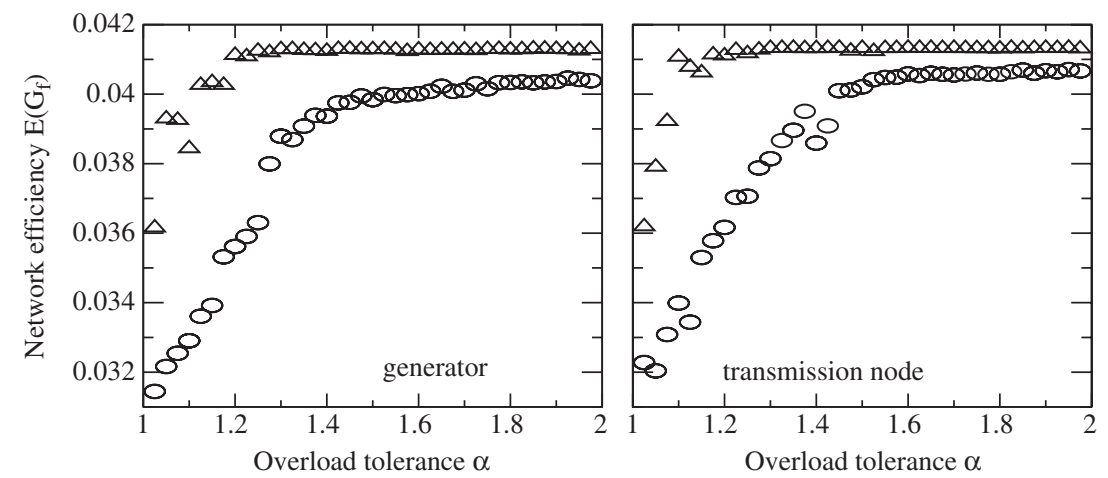

Fig. 3.4. Modelling cascading failure in the North American electrical power grid [301]. Global efficiency of the power grid after the removal of random (triangles) or high-load (circles) generators or transmission substations. As the overload tolerance $\alpha$ of the substations increases, the final efficiency approaches the unperturbed value. The random disruption curves were obtained by averaging over 10-100 individual removals. The load-based disruption curve is obtained by removing the highest load generator and transmission node, respectively. Reprinted figure with permission from Ref. [301]. (C) 2005 by the European Physical Society.

the North American power grid [225]. The reconstructed network contains $N=14,099$ substations and $K=19,657$ transmission (power) lines. The substations have been divided into three different groups: the generation substations set, whose elements produce electric power to distribute, the transmission substations set, whose elements transfer power along high voltage lines, and the distribution substations, whose elements distribute power to small, local grids.

Fig. 3.4 shows that, above a critical tolerance value of about 1.4, the removal of the highest loaded transmitter and generator substation has little effect on the overall network efficiency. However, at values of tolerance below the critical value, the global efficiency can be reduced by over 20 percent. For random removals, the critical value is near 1.18 in both figures. These results clearly indicate that the loss of nodes with high load causes a higher damage in the system than the loss of random nodes. 
The previous models give general indications of the complex and dramatic counter-effects due to the high degree of interconnection of electric power grids, but are too simplistic when it comes to model real blackouts. In Ref. [306] Carreras et al. have proposed a more realistic model for cascading failures blackouts in which the network nodes are characterized by an input power $P_{i}$, which is positive for generators and negative for customers/users, and each electric line connecting two nodes $i$ and $j$ is characterized by the power flow through it $w_{i j}$, and the impedance $z_{i j}$. The power flow through the network is calculated by solving the $d c$ power flow equations.

This is a standard way of analyzing a power transmission system [307], and it is equivalent to a linearized version of the more common problem of solving for the voltages and currents in a circuit. Cascading failures are simulated by associating to each generator a maximum power $P_{i}^{\max }$ it can supply, and to each line a maximum power flow $w_{i j}^{\max }$ it can stand. When an overloaded line $\left(w_{i j}>w_{i j}^{\max }\right)$ is found in the solution of the equations, the line impedance is multiplied by a large number, and the corresponding $w_{i j}^{\max }$ is divided by another large number, so that practically there is no power flow through the line. The model allows to examine cascading failures as the load power demand is increased, and to identify two types of critical points, those characterized by transmission line flow limits and those characterized by generator capability limits. In particular, it is found that operation near critical points can produce power law tails in the blackout size probability distribution, similar to those observed in the analysis of 15 years of North American blackout data [291,292]. Only ideal cases, as tree networks, and real networks with a small number of nodes $(\sim 100)$ have been considered in Ref. [306]. Large networks and the influence of the topology on the dynamics of the model have not been studied yet.

All the previous approaches to the modelling of overload breakdown avalanches consider a fixed network as the starting point. Of particular interest is the overload breakdown problem in time evolving networks. In fact, as the network structure changes, the load is redistributed, and if this is not accounted for, it may trigger a vertex breaking avalanche. Holme et al. have proposed a model for breakdowns triggered by changing vertices [286] or edges [287] load in an evolving network. The results show the presence of cascading failures, and those are more violent when the network growth is ruled by preferential rather than random attachment.

A different strategy has been followed in Ref. [308], where Wang and Xu have studied cascading failures in coupled map lattices (CML) [309]. The authors have proposed a model based on coupled logistic maps in the chaotic regime and a failure threshold mechanism, and have investigated different coupling topologies, including global coupling, small-world, and scale-free coupling. The breakdown of a single node is sufficient to trigger an entire network to collapse if the amplitude of the external perturbation on the single node is larger than a given threshold. Furthermore, it has been found that the threshold for a globally CML is much larger than that for a small-world or scale-free CML. This implies that cascading failures occur much easier in small-world and scale-free networks than in global coupling networks.

Large cascading failures are also present in social, economic [310] and biological systems [311]. In Ref. [312] the Bak-Sneppen model [313], a model of self-organized criticality for biological evolution, is studied on a scale-free topology, finding that, contrary to what is observed in regular lattices or in exponential graphs, the systems reach a stationary state characterized by the lack of a critical threshold barrier in the thermodynamic limit. Other models of self-organized criticality [314] have been studied on complex topologies in Refs. [315-317].

In Ref. [311] Watts has modelled cascades that manifest in social systems as collective actions, cultural fads or the diffusion of fashions and innovations. The model belongs to the class of problems known as binary decisions with externalities [318] and applies to all cases in which the factors involved in the decision are many, but the decision itself can be regarded as binary. The model considers a network in which each of the $N$ nodes (agents) observes the current states (either 0 or 1 ) of its $k$ neighbors and adopts state 1 if at least a threshold fraction $\phi$ of them are in state 1 . To model the dynamics of cascades, the population is initially all-off (state 0 ) and is perturbed at time $t=0$ by a small fraction $N_{1} \ll N$ of vertices that are switched to state 1 . The population then evolves at successive time steps with all vertices updating their states in random, asynchronous order according to the threshold rule above. Once a vertex has switched on, it remains on for the duration of the dynamics. To account for variations in knowledge, preferences, and observational capabilities across the population of decision-making agents, both individual thresholds and number of neighbors $k$ are allowed to be heterogeneous. Each agent is assigned a threshold drawn at random from a distribution $f(\phi)$ defined on the unit interval, and random graphs with a given $P(k)$ and average degree $\langle k\rangle$ have been considered. The results show that, in this model, the heterogeneity plays an ambiguous role. Indeed, increasingly heterogeneous thresholds make the system more vulnerable to global cascades, while an increasingly heterogeneous degree distribution makes it less vulnerable. 


\subsubsection{Congestion in communication networks}

Jamming phenomena are common in the Internet, where traffic is rerouted to bypass malfunctioning routers, eventually leading to an avalanche of overloads on other routers that are not equipped to handle extra traffic. This redistribution mechanism can result in a congestion regime with a large drop of the entire system performance [319,320].

A basic model of computer network traffic, that exhibits a phase transition from a free flow to a congested phase as a function of the packet generation rate, has been proposed by Ohira and Sawatari [321]. The model considers two classes of nodes: hosts, which can generate and receive messages, and routers which can only store and forward messages. At each time step, $p$ packets are created. Each of them is placed at a random host node $s$ (source), and is assigned a random destination address $t$ (target). The routers are capable of queuing an unlimited number of packets. At each time step, each node picks up the packet at the head of its queue (this type of queue is known as "first in first out", or FIFO) and decides which outgoing link is better suited to the packet destination. The routing strategy is based on shortest paths: packets are transferred, along the shortest path, from one router to another toward their final destinations. If more than one candidate of the next node exists, then there are two possibilities. In the so-called deterministic routing, the less congested node (the node to which the least number of packets has been forwarded so far) is selected as the next node. In the probabilistic routing there is also some tunable probability to send the packet to the most congested node. The model has been studied on simple network architectures as 2D lattices with hosts on the boundaries. As a function of $p$, it shows a sudden transition to a congestion state, where the congestion is measured in terms of the average travel time of packets. The relevant result is that the phase transition point, $p_{\mathrm{c}}$, depends on the routing strategy adopted. In particular, an appropriate randomness in path selection can shift the onset of traffic congestion, allowing to accommodate more packets in the network.

The model by Ohira and Sawatari has been generalized in different ways, for instance to consider a random location of hosts and routers [322], or to incorporate a packet creation rate regulated by the local congestion perceived by each node [323]. In particular, in Ref. [323], Valverde and Solé have shown that, as a result of the feedback between traffic congestion and packet release, the system self-organizes at the critical point of the transition. This is confirmed by the observation of large fluctuations and scaling in several relevant quantities, such as congestion duration lengths. Such a self-organized scenario is reinforced by a recent study suggesting that Internet fluctuations are a consequence of the internal dynamics of the system and not of the high variability injected by external sources [324]. In Ref. [325], the same authors have studied the model on a real Internet topology (that of Ref. [230]) showing that the Internet is able to route efficiently its inner flow of packets because of a special combination of routing rules and network architecture.

Routing strategies based on shortest paths require a global knowledge which is often not available. Traffic along geodesics may also create inconvenient queue congestions in some complex topologies as scale-free graphs, given that in such graphs the majority of shortest paths pass through hub nodes, while other nodes carry much less traffic [42]. A possible alternative is to consider local transport rules. Tadic̀, Rodgers and Thurner have studied the random diffusion (RD) of packets and have proposed an improved local search method, the so called cyclic search (CS) [326-328]. In the RD, the packet is delivered from the current node $i$ to one of its neighbors with equal probability, while the CS routing is based on searching the nearest and next-nearest neighbors of node $i$ for the target node of that packet. If found, the packet is moved to or towards its destination node; if instead the target $t$ is not within distance 2 from the original node $i$, then a random diffusion step is adopted.

Differently from the model by Ohira and Sawatari, here a packet can only be processed if the current queue size (the number of packets) at a selected neighbor node is below some maximal allowed buffer size $B$; otherwise, it waits for the next occasion to be processed. Either FIFO queues, but also "last-in-first-out" (LIFO) queues have been considered. The authors have implemented the model on various network structures: a scale-free tree, a randomly grown tree and the so-called Web graph, a directed graph grown by following the dynamic rules proposed in Ref. [156]. They have investigated the main properties of the dynamics (the time series of network load, and the transit and waiting time statistics) as a function the network topology, of the packet creation rate $p$, and of the kind of queue adopted. For small values of $p$, well below the jamming transition, the traffic is stationary and the transit times of packets follow a power-law distribution compatible with an anomalous superdiffusive process and with the broad distributions of travel times of packets empirically observed in the Internet traffic [329]. The power-law exponents depend on the graph topology and on the routing algorithm, while they are rather independent from the creation rate $p$ and from the type of queue adopted (the type of queuing plays a more important role for larger values of $p$ close to jamming, where waiting times of packets increase). Compared to RD, the CS routing improves the transport efficiency. However, the results depend crucially on the network topology: networks with higher organizational complexity, such as the Web graph, 
benefit more from an advanced routing algorithm [327]. The stationary regime is observed for a wide range of values of $p$, while before jamming at a critical creation rate $p_{\mathrm{c}}$, a crisis-like activity occurs with large variance in the network load [328].

In Ref. [330], Tadic̀ and Thurner have focused on how to improve the transport efficiency on scale-free networks with high structural complexity by using routings based on a local search within a pre-specified information horizon $r$ $(r>1)$ around the visited node ( $r=0$ corresponds to RD, and $r=1$ to $\mathrm{CS})$.

Routing mechanisms taking into account node congestion have been considered in the collection of models proposed in Refs. [320,331-333]. Here packets follow paths of minimum length from their origin $s$ to their destination $t$. At each time step, each packet moves from its current position $i$ to the next node in its path $j$ with a probability $q_{i j}$ which is called the quality of the channel and is defined in terms of the capabilities of the two nodes, $q_{i}$ and $q_{j}$ as $q_{i j}=\sqrt{q_{i} q_{j}}$. The quality of a channel is, thus, the geometric average of the capabilities of the two nodes involved so that, when one of the nodes has capability 0 , the channel is disabled. High qualities $\left(q_{i j} \simeq 1\right)$ imply that packets move easily, while low qualities $\left(q_{i j} \simeq 0\right)$ imply that it takes a long time for a packet to jump from one node to the next. It is assumed that $q_{i}=f\left(n_{i}\right)$, i.e. the capability of a node $i$ is a function of the number of packets $n_{i}$, currently at node $i$. The general function $f(n)=1$ for $n=0$ and $f(n)=n^{-\xi}$ for $n=1,2,3, \ldots$, with $\xi \geqslant 0$, has been considered. For $\xi>1$ $(\xi<1)$ the number of delivered packets decreases (increases) with the number of accumulated packets. For $\xi=1$ the number of delivered packets is independent of the number of accumulated packets. In 1D, 2D lattices and Cayley trees, a critical transition to congestion is found only for $\xi=1$, while for $\xi>1$ the transition to congestion is discontinuous and congestion nuclei arise.

The important role played by the topology in the congestion of communication networks is evident in Refs. [334-337,138].

Toroczkai et al. have compared the congestion in gradient networks generated by either random or scale-free substrates (see Section 2.3.4), finding that in the latter case the networks are less prone to jamming [137,138].

Holme has investigated the relationship between node betweenness and congestion in simple particle hopping models for traffic flow [334].

Echenique et al. have shown that local properties such as the clustering coefficient and degree-degree correlations have a great impact on the performance of traffic-aware models [335,336]. The latter authors have considered a more elaborated routing strategy that is, by itself, worthy of being analyzed in details. Let us suppose that a node $i$ is holding a packet whose destination is $t$. At each time step, the packet is then delivered from $i$ to the neighbor $j$ which minimizes the effective distance between $j$ and the target $t$, defined as

$$
\delta_{j}=h d_{j}+(1-h) n_{j}, j=1, \ldots, k_{i},
$$

where $d_{j}$ is the variation in the shortest path length between $i$ and $t$ if the packet follows the path through $j, n_{j}$ is the number of packets in the queue of node $j$, and $h$ is a tunable parameter that accounts for the degree of traffic awareness incorporated in the delivery algorithm. When $h=1$, the model recovers the usual shortest-path protocol similar to actual Internet routing mechanisms, while $h \neq 1$ is a traffic-aware scheme allowing to send packets through longer but less congested paths [336]. Other strategies are possible and have been considered in Refs. [335].

At each time step $p$ packets are introduced in the system, and the queues are FIFO with unlimited capacity. In Fig 3.5 we show the results obtained for a real network of the Internet at the autonomous system level, which is a scale-free network with $\gamma=2.2$ and $N=11,174$ nodes [336]. The number of packets $A(t)$ that have not reached their destinations at each time step $t$ are reported for different values of $p$ and $h$. For small packet creation rate $p$, both protocols allow for a stationary state. In this state, the system is able to balance the in-flow of packets with the flow of packets that reach their destinations. The stationary state, where no macroscopic signs of congestion is observed, corresponds to a free flow phase. The situation changes when the rate at which new packets are introduced increases, with the existence of a critical value $p_{\mathrm{c}}$ beyond which a congested phase shows up. For the standard protocol (Fig. 3.5a, dotted line), when $p>p_{\mathrm{c}}, A(t)$ grows linearly in time $\forall t$. On the contrary, for the traffic-aware algorithm, $A(t)$ is growing slowly at short times and then becomes steeper as time goes on with a constant slope (Fig. 3.5c). The phase transition from a free to a congested phase is characterized through the order parameter $\rho=\lim _{t \rightarrow \infty}(A(t+\tau)-A(t)) / \tau p$, where $\tau$ is the observation time. $\rho$ measures the ratio between the outflow and the inflow of packets during the time window $\tau$. $\rho$ equals 1 when no packet reaches its destination and 0 when an equilibrium is established, i.e., in the stationary state. 

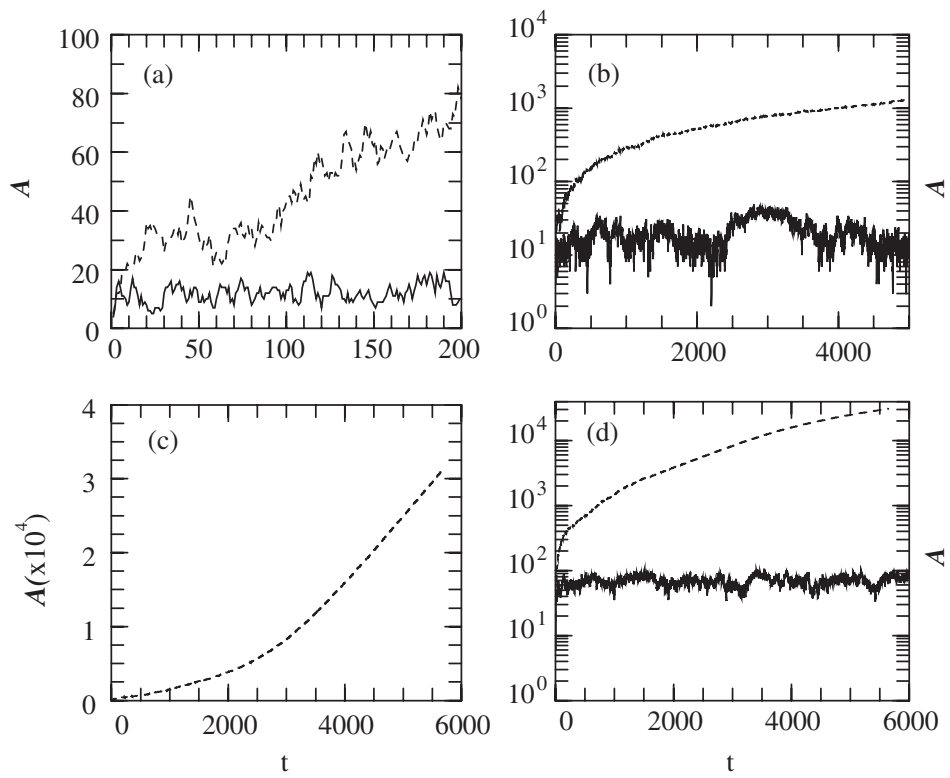

Fig. 3.5. Total number of active packets as a function of time steps in the model by Echenique et al. [336] with steady input of packets. Panels (a) and (b) correspond to the standard protocol, while (c) and (d) have been obtained for the traffic-aware routing with $h=0.85$. In each panel, the continuous line stands for subcritical values of $p$ ((a) and (b) $p=3.0$, (d) $p=8.0$ ) and the dotted line corresponds to $p>p_{\mathrm{c}}$ ((a) and (b) $p=4.0$, (c) and (d) $p=13.0$ ). Reprinted figure with permission from Ref. [336]. (C) 2005 by the European Physical Society.

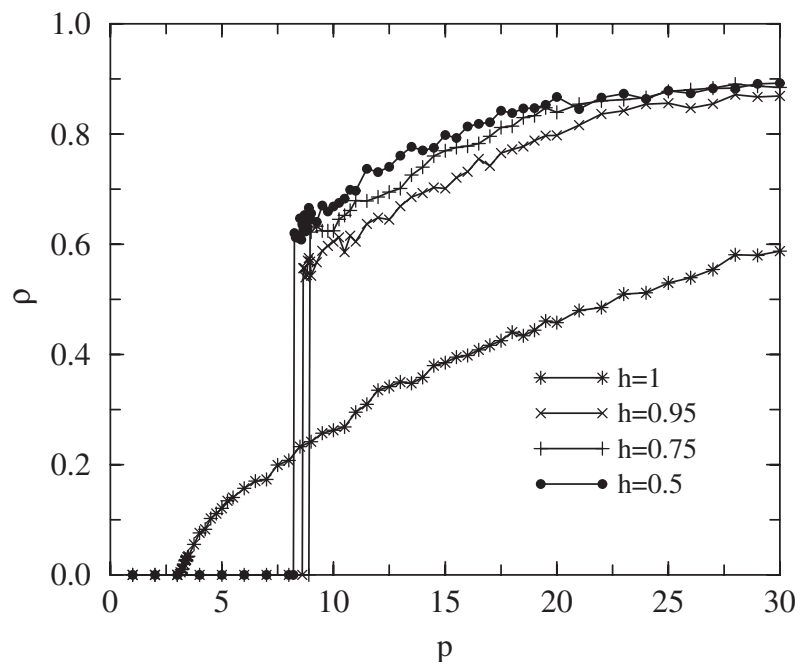

Fig. 3.6. Jamming transitions in the model by Echenique et al. [336]. The order parameter $\rho$ is reported as a function of $p$. Note that $h=1$ corresponds to the standard strategy in which traffic awareness is absent. As soon as traffic conditions are taken into account, the jamming transition is reminiscent of a first-order phase transition and the critical point shifts rightward. Figure taken from Ref. [336].

In Fig. 3.6 the system's phase diagram is shown. The dynamics of the system is characterized in both protocols by a critical point beyond which a macroscopic congestion arises. However, there are two radically different behaviors for the onset of traffic jams. In the standard protocol $(h=1)$, the critical point is small, $p_{\mathrm{c}}=3$, and the jamming transition is reminiscent of a second order phase transition. On the contrary, when $h \neq 1$, the critical point $p_{\mathrm{c}} \approx 9$ is distinctly larger than that for $h=1$, but the appearance of a congested phase turns out to be consistent with a first order phase transition, with a sharp jump of $\rho$ at the transition point. Moreover, the order of the transition for the latter protocol is independent of $h$ provided that $h \neq 1$. 
The results shown above indicate the richness of flow dynamics in networked systems and provide hints for the practical exploration of alternative algorithms. Finally, we note that various other models for congestion in communication networks have been explored (see for instance Refs. [337-340]).

\section{Spreading processes}

Cellular automata on complex topologies are systems in which each node of the network represents an agent that can be in only one of a finite number of states. Time is discrete, and at each time step, the next state of each agent is computed as a function of its state and of the states of its neighbors on the network. The formalism for cellular automata was introduced by von Neumann in the 1940s as a framework to study the process of reproduction [341]. Currently, cellular automata are considered as the simplest and nearly canonical form of a complex system [342]. In this Chapter we shall consider cellular automata describing spreading processes. In particular, in Section 4.1 we shall discuss epidemic spreading and, in Section 4.2, models for the diffusion of rumors. This two classes of processes are radically different.

Epidemic spreading has to do with the modelling for the spread of a particular infectious disease in a population, with the aim of reproducing the actual dynamics of the disease and designing the strategies to control and possibly eradicate the infection.

In rumor spreading, instead, one wants to spread the "rumors" as fast and efficiently as possible, not to prevent it from spreading. Practical examples are the design of protocols for data dissemination on the Internet, or strategies of marketing campaigns. In such cases, and in contrast to epidemic spreading, one is free to design the rules of the dynamics in order to reach the desired result. In both sections the attention will be focussed on the role of the network topology in determining the rate and patterns of the spreading process. We shall show that the inclusion of complex topologies in standard epidemic and rumors models radically changes the results previously established for random graphs and regular lattices.

\subsection{Epidemic spreading}

The study of epidemiological models is a subject of great interest that has attracted the attention of epidemiologists since long time ago. Mathematical epidemiology has grown exponentially since the middle of the 20th century, so that a tremendous variety of models has now been formulated, mathematically analyzed, and applied to field research [343-347]. Epidemiology modelling has been used in planning, implementing and evaluating various prevention, therapy and control programs.

On the other hand, physicists became interested in these kind of models when it was pointed out that epidemiological processes can be regarded as percolation like processes [348]. More recently, starting with the works by Pastor-Satorras and Vespignani [251,252], there has been a burst of activity on understanding the effects of the network topology on the rate and patterns of disease spread. Here, we discuss two classes of models that describe disease spreading through a population by contacts between infected and healthy individuals: the susceptible-infected-removed (SIR) and the susceptible-infected-susceptible (SIS) model. Different models and generalizations can be found in Refs. [344-347,349].

The theoretical approach to epidemic spreading is based on compartmental models, i.e. models in which the individuals in the population are divided into a set of different groups [344,345]. The SIR model describes diseases resulting in the immunization or death of infected individuals, and assumes that each individual can be in one of three possible states, susceptible (denoted by S), infected (I), or removed (R). Susceptible individuals are healthy persons that can catch the disease, if exposed to infected individuals. Once an individual catches the infection, it moves into the infected (and infective) class, and then, after some time, into the removed class. The term removed indicates that the individual cannot get the disease anymore (or passes it on), because it becomes immune to the disease (or dies). The model is based on two parameters, the transmission rate $\lambda$, and the recovery rate $\mu$. The individuals live at the sites of a given network, and the process proceeds via infection of neighbors with a rate $\lambda$ :

$$
S(i)+I(j) \stackrel{\lambda}{\longrightarrow} I(i)+I(j)
$$


(where $i$ and $j$ are two neighbors), and by recovery with rate $\mu$ :

$$
I(i) \stackrel{\mu}{\longrightarrow} R(i) .
$$

Not all diseases confer immunity to their survivors, so that an individual can catch the same disease more than once. Typical examples are tuberculosis and gonorrhea, or computer viruses in systems with no automatic updated antivirus programs [251,252]. All such cases are better described by the SIS model that considers only two states: susceptible (denoted by S) and infected (I). In the SIS model, susceptible individuals catch the infection, move into the infected/infectious state and become again susceptible after a period of time in which they recover. Finally, they are again exposed to the epidemic. The first reaction of the SIS model is the same as Eq.(4.1), while Eq.(4.2) must be replaced by the process $I(i) \stackrel{\mu}{\longrightarrow} S(i)$.

The two models have radically different dynamics following the initial introduction of a seed of infection into a susceptible population. In fact, the long term maintenance of the infection in a closed population is impossible in the SIR model, due to the depletion of susceptibles, as the epidemic spread through the population. Conversely, the SIS model is a model of endemic diseases: the same individual can be infected many times, so that the disease can persist indefinitely, circulating around the population. Consequently, the variables to be measured in the two cases are different. In the SIR model, as we shall see below, one is interested in the fraction of individuals (with respect to the total size of the susceptible population) who have caught the disease after it declined to zero. Whether the initial seed of infection (usually a single infected individual) can give rise or not to an infinite epidemic (i.e. an infection interesting a macroscopic part of the population) depends, of course, on the parameters $\lambda$ and $\mu$, more specifically on the ratio $\sigma=\lambda / \mu$. In particular, one wants to find the location of the epidemic transition, i.e. a critical value of the ratio $\left(\sigma=\sigma_{\mathrm{c}}\right.$ ) such that, for $\sigma<\sigma_{\mathrm{c}}$ no infinite epidemic is possible, while for $\sigma>\sigma_{\mathrm{c}}$ an infinite epidemic occurs with a finite probability. The analogous of the SIR epidemic transition in the SIS model is the critical value of $\sigma_{\mathrm{c}}$ that is at the boundary between values of the parameters in which the disease persists and those in which it does not.

Notwithstanding the above mentioned differences, recent studies have shown that the behavior of both SIS and SIR models on top of complex topologies is the same as far as the existence of critical points and the nature of the transition is concerned. For instance, in both models the spread of infections is tremendously strengthened on scale-free networks. For such a reason, here we shall mainly concentrate on the SIR model.

\subsubsection{The homogeneous mixing hypothesis}

In the first approximation, both the SIR and the SIS models are considered within the homogeneous mixing hypothesis [344], meaning that the individuals with whom a susceptible individual has contact are chosen at random from the whole population. This is a strong and questionable assumption since all the local details, such as geographical location, individual habits, and presence of community structures, are averaged out. On the other hand, it allows to write down the models in the form of systems of ordinary differential equations for the densities of individuals in the various classes. For instance, the SIR model in the homogeneous mixing hypothesis reduces to [343,344]:

$$
\begin{aligned}
\frac{\mathrm{d} s(t)}{\mathrm{d} t} & =-\lambda \bar{k} \rho(t) s(t), \\
\frac{\mathrm{d} \rho(t)}{\mathrm{d} t} & =-\mu \rho(t)+\lambda \bar{k} \rho(t) s(t), \\
\frac{\mathrm{d} r(t)}{\mathrm{d} t} & =\mu \rho(t),
\end{aligned}
$$

where $s(t), \rho(t), r(t)$ are, respectively, the density (i.e. the fraction) of susceptible, infected, and removed individuals at time $t$, and one of the equations is redundant because of the normalization condition $s(t)+\rho(t)+r(t)=1 \forall t$. System (4.3) can be interpreted as follows: infected individuals decay into the removed class at a rate $\mu$, while susceptibles individual become infected at a rate proportional to both the densities of infected and susceptible individuals. Here, $\bar{k}$ is the number of contacts per unit time that is supposed to be constant for the whole population (homogeneous mixing). Notice that $\lambda$ and $\mu$ are two fixed constant, so that no heterogeneity in the transmission rate between pairs of individuals and in the duration of the infection $(1 / \mu)$ is allowed. Another implicit assumption of the model is that the time scale of the disease is much smaller than the lifespan of individuals; consequently, terms accounting for the birth or natural death of individuals are not included in the equations. 
The most significant prediction of Eqs. (4.3) is the presence of a non-zero epidemic threshold $\lambda_{\mathrm{c}}$ [345]. If $\lambda>\lambda_{\mathrm{c}}$, the disease spreads and infects a finite fraction of the population. On the other hand, when $\lambda<\lambda_{\mathrm{c}}$, the total number of infected individuals (the so called epidemic incidence, defined as $r_{\infty}=\lim _{t \rightarrow \infty} r(t)$ ) is infinitesimally small in the limit of very large populations (the so-called thermodynamic limit [350]). The derivation of this result is simple. We consider an initial condition $s(0) \simeq 1, \rho(0) \simeq 0, r(0)=0$, i.e. a very small initial concentration of infected individuals, and without loss of generality we set $\mu=1$. By dividing the first of Eqs. (4.3) by the third, in order to cancel out $\rho(t)$, and then integrating with the initial conditions as indicated, we get $s(t)=\mathrm{e}^{-\lambda \bar{k} r(t)}$, and the following self-consistent equation for the epidemic incidence:

$$
r_{\infty}=1-\mathrm{e}^{-\lambda \bar{k} r_{\infty}} .
$$

While $r_{\infty}=0$ is always a solution of this equation, in order to have a non-zero solution, the following condition must be fulfilled:

$$
\left.\frac{\mathrm{d}}{\mathrm{d} r_{\infty}}\left(1-\mathrm{e}^{-\lambda \bar{k} r_{\infty}}\right)\right|_{r_{\infty}=0}>1
$$

Such a condition is equivalent to the constraint $\lambda>\lambda_{\mathrm{c}}$, with an epidemic threshold given by $\lambda_{\mathrm{c}}=\bar{k}^{-1}$. By using a Taylor expansion at $\lambda \simeq \lambda_{\mathrm{c}}$, it is then possible to prove that $r_{\infty} \sim\left(\lambda-\lambda_{\mathrm{c}}\right)$, above the epidemic threshold.

In the language of the physics of phase transitions [94,350], the threshold mechanism is completely equivalent to a critical point. In fact, in analogy with critical phenomena, $r_{\infty}$ can be considered as the order parameter of the phase transition, and $\lambda$ as the tuning parameter. The formula given above, describing the behavior $r_{\infty}$ as a function of $\lambda$ in the vicinity of $\lambda_{\mathrm{c}}$, defines one of the critical exponents of the phase transition, namely $\beta$. The numerical value obtained $(\beta=1)$ tells us that the homogeneous mixing hypothesis, in which we have assumed that the rate of contacts between infectious and susceptibles is constant, and independent of any possible source of heterogeneity, is indeed equivalent to a mean-field treatment of the model.

It is also worth remarking that the epidemic threshold is related to the basic reproductive rate $\mathscr{R}_{0}$, usually considered by the epidemiologists [344,351,352]. $\mathscr{R}_{0}$ is defined as the average number of secondary infections produced when one infected individual is introduced into a population where everyone is susceptible. Clearly, only if $\mathscr{R}_{0}$ is larger than unity, then the infection can sustain itself. In the case of homogeneous mixing, the basic reproductive rate is given by $\mathscr{R}_{0}=\lambda \mu^{-1} \bar{k}$, with the condition $\mathscr{R}_{0}>1$ obviously defining the threshold in the spreading rate $\lambda_{\mathrm{c}}=\mu \bar{k}^{-1}[346,352]$.

\subsubsection{Uncorrelated networks}

The assumption of homogeneous mixing is a useful starting point for theoretical studies and allows for further elaborations, as e.g. the inclusion of age classes [344]. Tests against data, however, confirm that a crucial element that needs to be considered is the network topology. For instance, most sexually transmitted diseases cannot be understood without acknowledging the importance of spatial structures for disease transmission, and the marked heterogeneity in degrees of sexual activity within the overall population $[344,351,353]$. To deal with populations which are not perfectly mixed, it is natural to consider the individuals as the nodes of a regular lattice, or of any kind of graph, that represents the transmission network. In such a case, the transmission of the disease proceeds through contacts between infected and susceptible nodes that are neighbors (i.e. directly connected by a link) on the network.

As shown by Grassberger in Ref. [348], the problem of whether in the SIR model on a given network a single infected site can give rise to an infinite epidemic can be mapped into a percolation problem on the same network. In fact, the probability of occurrence of an infinite epidemic is exactly equal to the chance that the original infected node belongs to an infinite cluster. The SIR model defined in Eqs. (4.1)-(4.2) corresponds to neither site nor bond percolation [281]. Eq. (4.2) implies that infective individuals remain infective for a time that is exponentially distributed with mean value $T=1 / \mu$. If one assumes that the times of illness are all equal to $T$, then the probabilities that the infection is transmitted between two generic neighboring nodes are all independent and equal to:

$$
p=1-\lim _{\delta t \rightarrow 0}(1-\lambda \delta t)^{T / \delta t}=1-\mathrm{e}^{-\lambda / \mu} .
$$

That is precisely the problem of bond percolation on the same network, with bond probability $p$ for the bonds to be unbroken [348]. The connection between disease spreading and percolation can be extended to other models. 
For instance, the case in which $\lambda$ and $\mu$, instead of being fixed, are independent random variables has been considered in Refs. [354,355]. The mapping with percolation models has been exploited in the SIR model to extract the critical exponents for regular two-dimensional lattices [348,354]. It also allows to derive exact solutions for the presence and the size of an epidemic in random graphs with arbitrary degree distributions [355], based on the fact that percolation problems can be solved in random graphs by means of the generating functions formalism [124,276].

Heterogeneous graphs with highly skewed degree distributions are particularly important to describe real transmission networks. For example, in the case of sexual contacts, which are responsible for the diffusion of sexually transmitted diseases, the degree distribution has been found to follow a power-law [356,357]. For this reason, here we will consider in detail the properties of the SIR model on uncorrelated heterogeneous graphs with a generic degree distribution $P(k)$ and a finite average connectivity $\langle k\rangle$. Our approach will follow Refs. [251,252,358], and complements the approach of the generating function formalism [355], being able to give information on the time evolution of the epidemic outbreak. We are now interested in the time evolution of the quantities $s_{k}(t), \rho_{k}(t)$ and $r_{k}(t)$, which are the density of susceptible, infected, and removed nodes of connectivity $k$ at time $t$, respectively. The normalization condition, valid at each time $t$, is now:

$$
s_{k}(t)+\rho_{k}(t)+r_{k}(t)=1 .
$$

Global quantities, such as the epidemic incidence, are expressed by an average over the various connectivity classes: $r_{\infty}=\lim _{t \rightarrow \infty} r(t)$, with $r(t)=\sum_{k} P(k) r_{k}(t)$. At the mean-field level, these densities satisfy the same set of coupled differential equations ass in Eqs. (4.3), but differentiated by connectivity classes:

$$
\begin{aligned}
\frac{\mathrm{d} s_{k}(t)}{\mathrm{d} t} & =-\lambda k s_{k}(t) \Theta(t), \\
\frac{\mathrm{d} \rho_{k}(t)}{\mathrm{d} t} & =-\rho_{k}(t)+\lambda k s_{k}(t) \Theta(t), \\
\frac{\mathrm{d} r_{k}(t)}{\mathrm{d} t} & =\rho_{k}(t),
\end{aligned}
$$

where we have set $\mu=1$, and where the factor $\Theta(t)$ represents the probability that any given link points to an infected site and is given by [251,252]

$$
\Theta(t)=\frac{\sum_{k} k P(k) \rho_{k}(t)}{\langle k\rangle} .
$$

In this approximation we are neglecting the connectivity correlations in the network, i.e., the probability that a link points to an infected node is considered independent of the connectivity of the node from which the link is emanating.

Eqs. (4.8), combined with the initial conditions $\rho_{k}(0)=\rho_{k}^{0}, s_{k}(0)=1-\rho_{k}^{0}$ and $r_{k}(0)=0$, completely define the SIR model on any complex network with connectivity distribution $P(k)$. We will consider, in particular, the case of a homogeneous initial distribution of infected nodes, $\rho_{k}^{0}=\rho^{0}$. In this case, in the limit $\rho^{0} \rightarrow 0$, we can substitute $\rho_{k}(0) \simeq 0$ and $s_{k}(0) \simeq 1$. Under this approximation, the first of Eqs. (4.8) can be directly integrated, yielding $s_{k}(t)=\mathrm{e}^{-\lambda k \phi(t)}$, where we have defined the auxiliary function $\phi(t)=\int_{0}^{t} \Theta\left(t^{\prime}\right) \mathrm{d} t^{\prime}=1 /\langle k\rangle \sum_{k} k P(k) r_{k}(t)$, and, in the last equality, we have made use of the definition (4.9). It is worth remarking that the above equations are similar to those obtained in the case of HIV dynamics in heterogeneous populations [353]. With these equations, one can show [358] that, in the infinite time limit, i.e. at the end of the epidemics, the condition $1 /\langle k\rangle \sum_{k} k P(k)(\lambda k)=\lambda\left\langle k^{2}\right\rangle /\langle k\rangle>1$, defines the epidemic threshold:

$$
\lambda_{\mathrm{c}}=\frac{\langle k\rangle}{\left\langle k^{2}\right\rangle}
$$

below which the epidemic incidence is null, and above which it attains a finite value. Eq. (4.10) tells us that the threshold is inversely proportional to the connectivity fluctuations $\left\langle k^{2}\right\rangle$. This is the same picture gathered for percolation like processes and is at the root of the absence of critical thresholds in these kind of processes.

For networks in which $\left\langle k^{2}\right\rangle<\infty$, the threshold has a finite value and we are in the presence of a standard phase transition. On the other hand, in networks with strongly fluctuating connectivity distribution, for instance in scalefree networks with $2<\gamma \leqslant 3$, the unbounded fluctuations of the connectivity distribution imply a vanishing epidemic 
threshold for increasing network sizes, since $\left\langle k^{2}\right\rangle \rightarrow \infty$ when $N \rightarrow \infty$. The absence of any intrinsic epidemic threshold can be understood by noticing that, for heterogeneous networks, the basic reproductive number $\mathscr{R}_{0}$ is equal to $\mathscr{R}_{0}=\lambda \mu^{-1}\left\langle k^{2}\right\rangle /\langle k\rangle$ [344,351-353]. In scale-free networks the divergence of $\left\langle k^{2}\right\rangle$ leads to a $\mathscr{R}_{0}$ that always exceeds unity, whatever the spreading rate $\lambda$ is. This ensures that epidemics always have a finite probability to survive indefinitely.

Historically, the absence of an epidemic threshold in scale-free networks was first reported in the SIS model by Pastor-Satorras and Vespignani [251,252]. The authors derived theoretically, by a dynamical mean-field approximation similar to what we have used here for the SIR model, an epidemic threshold formula equal to Eq. (4.10). This result was validated numerically by showing that the survival probability of one infected node in scale-free networks has a finite value for large enough sizes of the systems under study, even for small spreading rates. Furthermore, it was shown [251,252] that this is not the case for random graph topologies, where the classical picture of a finite threshold still holds.

Based on a complementary approach (the generating function formalism) similar results were obtained in Ref. [355]. The main difference between these methods is that the mean field approach provides the time evolution of the epidemic outbreak whereas the generating function approach allows for an exact solution of the SIR model.

It is worth remarking that real networks have always a finite size $N$ and thus an effective threshold, depending on $\langle k\rangle$ and $\left\langle k^{2}\right\rangle$, that can be easily calculated as a function of $N$. This apparent threshold however is not an intrinsic quantity and it is extremely small for systems with large enough $N[359,360]$. Moreover, this threshold is smaller than that of a random graph with the same size.

In Refs. [361,362] the authors have studied the best immunization strategies on scale-free networks, showing that networks with scale-free topology do not acquire global immunity even in the presence of unrealistically high density of randomly immunized nodes. Conversely, a targeted immunization based on the node connectivity can restore a finite epidemic threshold and potentially eradicate a virus. This result gives also important indications on the best public health strategies to adopt in order to control and eradicate sexually transmitted diseases (as the HIV [363]). The real problem here is that sexual promiscuous individuals are not always easy to identify. Cohen et al. have suggested a method to circumvent this problem based on the vaccination of random acquaintances of random chosen individuals [364]. This strategy is based on the fact that the probability of reaching a particular node by following a randomly chosen edge is proportional to the nodes degree (see Eq. (3.1)), requires no global knowledge of the node degrees, and is proven to be more effective than random vaccination. Further elaboration on local strategies for vaccination can be found in Ref. [365].

Other complex topologies have been considered. Networks with a high clustering coefficient, as those constructed by the structured scale-free model (see Section 2.3.5), show a finite epidemic threshold even for a diverging second moment of the degree distribution [366]. In this case, the presence of a finite epidemic threshold was motivated by the high clustering, which prevents the spreading of the infection. However, later on it was argued that the argument was somehow misled because structured scale-free networks do not possess the small-world property [173]. The basic assumption of the dynamical mean field approach is that all vertices within a given degree class can be considered statistically equivalent, therefore the results are not applicable to networks in which a distance or time ordering is present.

Another interesting contribution to the topic comes from Volchenkov et al., who introduced a network model having scale-free properties and showing persistent infections at any spreading rate $\lambda>0$ and for any $\gamma>1$ [367]. Such alternative flexible model is inspired by the principle of evolutionary selection of common large-scale structure in biological networks. The results were also analytically proved by means of a more sophisticated mean field approximation, which incorporates connectivity matrices between nodes having $k$ and $k^{\prime}$ degrees [367].

Finally, epidemic spreading in weighted networks has only recently started to be addressed [223,224].

\subsubsection{Correlated networks}

The lack of an epidemic threshold in assortative networks for the SIR model has been verified numerically in Ref. [368]. The authors introduced a method that allows to study the spreading of diseases in correlated graphs without implicitly generating the networks, which is the first problem to overcome when dealing with artificial networks with correlations. In particular, it was found that the qualitative behavior is the same as for uncorrelated networks, although there are some important quantitative differences. While the likelihood of an epidemic outbreak is not modified when taking into account positive correlations, the epidemic incidence is smaller than in networks with no correlations. 
In large social networks this may lead to a difference of $15 \%$ to $20 \%$ of infected people for moderate values of the spreading rate. Besides, the diseases are longest lived in assortative networks.

The SIS model on top of graphs with degree correlations has been considered in Refs. [24,369]. The authors have obtained an epidemic threshold $\lambda_{\mathrm{c}}=1 / \Lambda_{\max }^{\prime}$, where $\Lambda_{\max }^{\prime}$ is the largest eigenvalue of the matrix $C_{k k^{\prime}}^{\prime}=k P\left(k^{\prime} \mid k\right)$. The approach followed is quite similar to the one presented in Section 3.1.3 for site percolation with the remark that $C_{k k^{\prime}}^{\prime}$ is different from $C_{k k^{\prime}}$ defined in Eq. (3.17) [280]. In fact, if $y_{k}$ is an eigenvector of $C_{k k^{\prime}}^{\prime}$ corresponding to the eigenvalue $\Lambda^{\prime}$, then $y_{k} / k$ is an eigenvector of $C_{k k^{\prime}}^{\prime \prime}=k^{\prime} P\left(k^{\prime} \mid k\right)$ corresponding to the same eigenvalue. This last matrix is that of Eq. (3.17), if one replaces $k^{\prime}$ by $k^{\prime}-1$. However, this subtle difference makes the SIS and site percolation different. By computing the largest eigenvalue of $C_{k k^{\prime}}^{\prime \prime}$ for the case of a disassortative graph (as the one defined in Eq. (3.26)), and taking the limit $\left\langle k^{2}\right\rangle \gg 1$ one gets [280]:

$$
\Lambda_{\max }^{\prime} \approx \frac{\sum_{k}\left(1-g_{k}\right) k^{2} P(k)}{\sum_{k^{\prime}}\left(1-g_{k^{\prime}}\right) k^{\prime} P\left(k^{\prime}\right)},
$$

where $g_{k}$ is again a decreasing function of $k$. In this case, independently of the form of $g_{k}$, the divergence of the second moment of the degree distribution implies the divergence of $\Lambda_{\max }^{\prime}$. Moreover, the same conclusion is obtained if $g_{k}$ is an increasing function of $k$. This in contrast to the conclusions drawn in Section 3.1.3 for site percolation in correlated networks, where it was shown that the divergence of the second moment of the distribution is not a sufficient condition for a network to be robust under random failures. This essential difference between the SIS model and site percolation on correlated networks is rooted in the existence of an additional dimension in the SIS model, given by the time evolution of the density of infected sites. The same result can be obtained by directly analyzing the SIS model [369].

\subsubsection{Fluctuating diseases}

Non-equilibrium diseases are those in which outbreaks fluctuate in time. Examples of real epidemics showing this kind of behavior include measles, smallpox, chickenpox, syphilis and dengue. Approaches to fluctuating outbreaks and stochastic epidemics have been proposed within the recent literature (see for instance [370]), pointing out that outbreaks stem from stochastic perturbations in epidemic or population parameters. However, models where topological and dynamical complexity coexist in an epidemic spreading scenario have not been studied in details so far. This is due in part to the inherent complex patterns of interconnections between individuals, but also to the intrinsic nonlinearity of the problem.

Some exceptions are the works in Refs. [371,372]. In particular, Vannucchi and Boccaletti have studied the dynamics of an epidemiological disease spreading on top of a complex network of individuals [371]. The main contribution consists of introducing an excitable dynamics in the local behavior of the nodes. Specifically, each site $(i, j)$ of a 2D lattice is associated with two dynamical variables, an activator variable $u_{i, j}$ and an inhibitor variable $v_{i, j}$. The local dynamics at site $(i, j)$ is given by the standard Fitz-Hugh-Nagumo (FHN) equations:

$$
\begin{aligned}
& \partial u_{i, j} / \partial t=-u_{i, j}\left(a-u_{i, j}\right)\left(1-u_{i, j}\right)+v_{i, j}+C_{i, j}, \\
& \partial v_{i, j} / \partial t=e u_{i, j}
\end{aligned}
$$

where $a, e$ are two parameters of the model, while the topological complexity is contained in the network coupling term $C_{i, j}$.

The dynamics of the FHN model is well known: the point here is that the dynamical regimes of the model can be related to epidemic states. In this way, when the system is lying on the stationary or quiescent state, a proper perturbation activates the dynamics. Thus, such a state is associated with the susceptible class in epidemiological models. The second phase can be distinguished in the totally refractory state. In this state the system is practically insensitive to perturbations of any size. This state is then associated with the refractory period of individuals during the evolution of their disease where they cannot be further infected by the same disease. Finally, the recovery dynamics toward the stationary state can be though of as a relative refractory state. Note that, in this latter state, another outbreak can be induced for perturbations larger than the ones necessary in the stationary state, nicely modelling the fact that an individual who is recovering from a recent disease is somehow more robust against the same infection. 
The authors reported that different dynamics states are possible depending on whether or not the topology of the underlying network is a $2 D$ regular (local) network or a $2 D$ small-world network. Moreover, the chaotic regime is characterized by a spatial evolution of the epidemics wherein macroscopic domains of infected and susceptible individuals coexist and interact.

\subsection{Rumor spreading}

As seen before, the inclusion of complex topologies in standard epidemic models radically changes the results previously established for random and regular graphs. This might be bad news for epidemiologists, and those fighting real and computer viruses. On the other hand, in a number of important technological and commercial applications, rather than preventing an outbreak, it is desirable to spread the "epidemic" as fast and as efficiently as possible.

Important examples of such applications are epidemic (or rumor-based) protocols for data dissemination and resource discovery on the Internet [99,373-375], and marketing campaigns using rumor-like strategies (viral marketing). The above applications, and their dynamics, have passed almost unnoticed [376,377] to the physics community working on complex networks despite the fact that they have been extensively studied by computer scientists and sociologists [375,378]. The problem here consists of designing an epidemic (or rumor-mongering) algorithm in such a way that the dissemination of data or information from any node of a network reaches the largest as possible number of remaining nodes. The main difference with respect to epidemic models is that one is free to design the rules of the dynamics in order to reach the desired result, instead of having to model an existing process. Furthermore, in a number of applications, such as peer-to-peer file sharing systems [99] built on top of the Internet and grid computing [379], the connectivity distribution of the node can also be changed in order to maximize the performance of such protocols.

The standard rumor model is the so-called DK model, proposed several decades ago by Daley and Kendal [380]. Recently, several authors [376,377,381,382] have explored this model and slight variations of it on top of complex topologies. The basic DK rumor model is defined as follows. Each of the $N$ elements of the network can be in one of three possible states. Following the original terminology [378], these three classes correspond to ignorant (denoted by $\mathrm{I})$, spreader $(\mathrm{S})$ and stifler $(\mathrm{R})$ nodes. Ignorants are those individuals who have not heard the rumor and hence they are susceptible to be informed. The second class comprises active individuals that are spreading the rumor. Finally, stiflers are those who know the rumor but that are no longer spreading it.

In epidemiological models, the time evolution of the different classes is only determined by the dynamics of the individuals and does not depend on the state of their neighbors, except in the creation term, i.e., the interaction infected-susceptible. On the other hand, the rumor dynamics is driven by direct contacts between individuals of different classes. As we will see, this difference in the annihilation term is at the root of a quite different qualitative picture. The dynamics of the model, at each time step, is ruled by the following transitions:

$$
\begin{aligned}
& I(i)+S(j) \stackrel{\lambda}{\longrightarrow} S(i)+S(j), \\
& S(i)+S(j) \stackrel{\alpha}{\longrightarrow} R(i)+S(j), \\
& S(i)+R(j) \stackrel{\alpha}{\longrightarrow} R(i)+R(j),
\end{aligned}
$$

where $i$ and $j$ are two neighbors. The spreading process evolves by contacts between spreaders and ignorants. When an ignorant meets a spreader, it turns itself into a new spreader at a rate $\lambda$. The decay of spreading may be due to a process of "forgetting", or because spreaders learn that the rumor has lost its "news value". In the equations above we have assumed this latter hypothesis as the most plausible so that spreaders become stiflers with probability $\alpha$ if they are in contact with another spreader or a stifler. As one is free to design the rumor in such a way that the fraction of the population which ultimately learns the rumor is the maximum possible, it is also assumed that contacts of the type spreader-spreader are directed, that is, only the contacting individual loses the interest in propagating the rumor further.

In the homogeneous mixing hypothesis, the DK model can be described in terms of the densities of ignorants, spreaders, and stiflers, $i(t), s(t)$, and $r(t)$, respectively, as a function of time. The mean-field rate equation [376,382] 
for the evolution of the three densities satisfy the following set of coupled differential equations:

$$
\begin{aligned}
\frac{\mathrm{d} i(t)}{\mathrm{d} t} & =-\lambda\langle k\rangle i(t) s(t), \\
\frac{\mathrm{d} s(t)}{\mathrm{d} t} & =\lambda\langle k\rangle i(t) s(t)-\alpha\langle k\rangle s(t)[s(t)+r(t)], \\
\frac{\mathrm{d} r(t)}{\mathrm{d} t} & =\alpha\langle k\rangle s(t)[s(t)+r(t)],
\end{aligned}
$$

with the initial conditions $i(0)=(N-1) / N, s(0)=1 / N$ and $r(0)=0$. Besides, we have the normalization condition $i(t)+s(t)+r(t)=1$. The first equation states that the density of spreaders increases at a rate proportional to the spreading rate $\lambda$, to the average number of contacts of each individual $\langle k\rangle$, and to the densities of ignorant and spreader individuals, $i(t)$ and $s(t)$, respectively. On the other hand, spreaders decay into the stifler class at a rate $\alpha\langle k\rangle$ times the density of spreaders and of non-ignorant individuals $1-i(t)=s(t)+r(t)$.

The system of differential equations (6.2)-(4.19) can be analytically solved in the infinite time limit when $s(\infty)=0$. Using the normalization condition, recalling that $f=\int_{0}^{\infty} s(t) \mathrm{d} t=\lim _{t \rightarrow \infty} r(t)=r_{\infty}$, and introducing the new variable $\beta=1+\lambda / \alpha$ we get the transcendental equation:

$$
r_{\infty}=1-\mathrm{e}^{-\beta r_{\infty}}
$$

Eq. (4.20) always admits the trivial solution $r_{\infty}=0$, but at the same time it also has another physically relevant solution for all values of the parameters $\lambda$ and $\alpha$. This can be easily appreciated since the condition:

$$
\left.\frac{\mathrm{d}}{\mathrm{d} r_{\infty}}\left(1-\mathrm{e}^{-\beta r_{\infty}}\right)\right|_{r_{\infty}=0}>1
$$

reduces to $\lambda / \alpha>0$. Thus, the behavior of the system under the dynamical rules defined above is strikingly different from that of the equivalent epidemic model (that would correspond to the assumption of a decaying spreading rate independent of $s-r$ and $s-s$ interactions), because the fraction $f$ of the population who learns the rumor when the process dies is always above 0.8. That is, there is no "rumor threshold", contrarily to the case of epidemic spreading. The difference does not come from any difference in the growth mechanism of $s(t)$ — the two are actually the same-, but from the disparate rules for the decay of the spreading process.

On the other hand, this result also points out that a mathematical model for the spreading of rumors can be constructed in many different ways, being the annihilation algorithm the most relevant for practical implementations [381].

For more complex topologies, we have to rely necessarily on numerical simulations. Initially, $\psi(0)=(N-1) / N$, $\phi(0)=1 / N$, and $r(0)=0$, i.e., we start from a single spreader who is willing to spread the update through the network. At every time step, each of the $\phi N$ spreaders randomly contacts up to its full number of neighbors, provided that they remain in the $\phi$ class. The dynamical rules of the model are applied in parallel. Other kind of local rules can also be implemented. Finally, several quantities of interest are obtained in the same way as done in epidemic spreading, namely the final density $r(\infty)$ of nodes that have got the rumor when the process dies out, and the temporal evolution of the different classes.

However, the study of rumor processes by means of Monte Carlo simulations is a lengthy and cumbersome task, especially if we are interested in exploring many variants in order to design an efficient rumor propagation. Therefore, the development of easier to handle numerical techniques is a highly useful issue. One of these methods was recently proposed in [368], where a numerical technique to deal with the mean-field rate equations appearing in epidemic-like models was introduced. It solves the differential equations by calculating the passage probabilities for the different transitions. The main advantage is that one does not have to rely on MC simulations and thus memory requirement for large system sizes is not a limitation. Besides, we do not have to generate any network. Instead, a sequence of integers distributed according to the desired connectivity distribution $P(k)$ is produced. The numerical procedure consists of the following steps. At each time step until the end of the rumor spreading process, the following tasks are performed:

1. Identify from the mean-field rate equations the transition probabilities from one state into the following one, that is, from the $i$ class to the $s$ class, $W_{i \rightarrow s}$, and finally to the $r$ class, $W_{s \rightarrow r}$. 
2. Calculate the mean time interval $\tau$ for one transition to occur. This is determined as the inverse of the sum of all the transition probabilities; $\tau=1 /\left(W_{i \rightarrow s}+W_{s \rightarrow r}\right)$.

3. Stochastically decide what transition will actually take place. This is done by deciding that the probabilities for both transitions are given by $\prod_{i \rightarrow s}=W_{i \rightarrow s} \tau$ and $\prod_{s \rightarrow r}=W_{s \rightarrow r} \tau$, respectively, materializing the choice by generating a random number between 0 and 1 .

This numerical recipe does not depend on the topological features of the network on top of which the rumor dynamics is taking place. Indeed, all the topological information, including correlations, enters in the computation of the transition probabilities. To be specific, let us derive the transition probabilities for two different networks, an homogeneous one and a random scale-free network. For the homogeneous case we have that the transition probabilities are the same for all the elements within a given class $(i, s$ or $r$ ), irrespective of their actual connectivities. Then, from Eqs. (6.2)-(4.19) one gets

$$
\begin{aligned}
& W_{i \rightarrow s}(t)=N \lambda\langle k\rangle i(t) s(t), \\
& W_{s \rightarrow r}(t)=N \alpha\langle k\rangle s(t)[s(t)+r(t)],
\end{aligned}
$$

for the transitions from the ignorant to the spreader class and from the spreader to the stifler class, respectively.

The heterogeneity of the connectivity distribution inherent to SF networks makes it necessary to take into account that nodes could not only be in three different states, but also they belong to different connectivity classes $k$. Denoting by $i_{k}(t), s_{k}(t)$, and $r_{k}(t)$ the densities of ignorants, spreaders and stiflers with connectivity $k$, with $i_{k}(t)+s_{k}(t)+r_{k}(t)=1$, we have that the rate equations now read as

$$
\begin{aligned}
\frac{\mathrm{d} i_{k}(t)}{\mathrm{d} t} & =-\lambda k i_{k}(t) \sum_{k^{\prime}} \frac{k^{\prime} P\left(k^{\prime}\right) s_{k^{\prime}}(t)}{\langle k\rangle}, \\
\frac{\mathrm{d} s_{k}(t)}{\mathrm{d} t} & =\lambda k i_{k}(t) \sum_{k^{\prime}} \frac{k^{\prime} P\left(k^{\prime}\right) s_{k^{\prime}}(t)}{\langle k\rangle}-\alpha k s_{k}(t) \sum_{k^{\prime}} \frac{k^{\prime} P\left(k^{\prime}\right)\left[s_{k^{\prime}}(t)+r_{k^{\prime}}(t)\right]}{\langle k\rangle}, \\
\frac{\mathrm{d} r_{k}(t)}{\mathrm{d} t} & =\alpha k s_{k}(t) \sum_{k^{\prime}} \frac{k^{\prime} P\left(k^{\prime}\right)\left[s_{k^{\prime}}(t)+r_{k^{\prime}}(t)\right]}{\langle k\rangle},
\end{aligned}
$$

where $P(k)$ is the connectivity distribution of the nodes and $\sum_{k^{\prime}} k^{\prime} P\left(k^{\prime}\right) s_{k^{\prime}}(t) /\langle k\rangle$ is the probability that any given node points to a spreader. We start from a randomly selected spreader and all the remaining nodes in the ignorant class. The summation in Eq. (4.25) stands for the probability that a node points to a spreader or a stifler. The respective transition probabilities (which depend on $k$ as well) are:

$$
\begin{aligned}
& W_{i \rightarrow s}(t, k)=\lambda k N P(k) i_{k}(t) \sum_{k^{\prime}} \frac{k^{\prime} P\left(k^{\prime}\right) s_{k^{\prime}}(t)}{\langle k\rangle}, \\
& W_{s \rightarrow r}(t, k)=\alpha k N P(k) s_{k}(t) \sum_{k^{\prime}} \frac{k^{\prime} P\left(k^{\prime}\right)\left[s_{k^{\prime}}(t)+r_{k^{\prime}}(t)\right]}{\langle k\rangle},
\end{aligned}
$$

where all the topological information is contained. Finally for the mean time interval we have at each time step

$$
\tau=\frac{1}{W_{i \rightarrow s}(t)+W_{s \rightarrow r}(t)},
$$

with $W_{i \rightarrow s}(t)=\sum_{k} W_{i \rightarrow s}(t, k), W_{s \rightarrow r}(t)=\sum_{k} W_{s \rightarrow r}(t, k)$ and $t=\sum_{j}^{i-1} \tau_{j}$. At this point, the identification of what transition takes place and which connectivity class is affected proceeds as defined in step 3 above.

The numerical exploration of rumor spreading models has been performed $[381,382]$ in the context of communication networks, where epidemic-like information dissemination is thought to be efficient and scalable to larger system sizes. Moreover, several variants for the annihilation terms allow for different outcomes. This in fact means that, contrary to epidemic modelling, rumor processes can be tailored depending on the specific application. In this respect, there are still many issues to be addressed such as the influence of correlations, and dynamical rewiring. But we already know that even for a very poorly designed rumor, the final fraction of stiflers is finite at variance with epidemiological models. 
Future works must optimize the information dissemination in order to optimize the trade-off between the fraction of stiflers and the resources invested in the spreading process.

\section{Synchronization and collective dynamics}

The emergence of collective and synchronized dynamics in large networks of coupled units has been investigated since the beginning of the nineties in different contexts and in a variety of fields, ranging from biology and ecology [371,383,384], to semiconductor lasers [385-388], to electronic circuits [389,390].

This chapter aims at reviewing some different techniques that have been proposed for assessing the propensity for synchronization of a given networked system. We will describe the main applications, especially in the view of selecting the optimal topology in the coupling configuration that provides enhancement of the synchronization features. This approach, indeed, has defined a new framework for the understanding of several relevant situations, where networks of dynamical systems experience mutations in their architecture as a function of time, or they evolve themselves as dynamical entities, as we shall see in Section 7.3. The chapter ends with a review of the other collective dynamics that have been observed and studied in complex networks.

\subsection{Introduction to synchronization}

Synchronization is a process wherein many systems (either equivalent or non-equivalent) adjust a given property of their motion due to a suitable coupling configuration, or to an external forcing. The origin of such word comes from a greek root ( $\sigma \grave{v} \gamma \quad \chi \rho o ́ v o \varsigma$ which means "to share the common time").

Historically, synchronization phenomena have been actively investigated since the earlier days of physics. Already in the 17th century, indeed, Christian Huygens discovered that two pendulum clocks hanging at the same beam were able to perfectly synchronize their phase oscillations [391]. Other early found examples of synchronous motion include the synchronized lightning of fireflies, and the properties of adjacent organ pipes, which, in some cases, can almost reduce one another to silence, or speak in absolute unison.

Initially, the attention was mainly devoted to synchronization of periodic systems, while recently the search for synchronization has moved to chaotic systems [392]. When chaotic elements are coupled, many different synchronization phenomena can take place, from complete or identical synchronization [393-395], to phase [396,397] and lag synchronization [398], generalized synchronization [399,400], intermittent lag synchronization [398,401], imperfect phase synchronization [402], and almost synchronization [403].

Complete synchronization (or identical synchronization) is the simplest form of synchronization, and consists in a perfect hooking of the trajectories of identical chaotic systems in the course of the time. Generalized synchronization, instead, considers different systems and associates the output of one system to a given function of the output of another system [399,400]. Coupled non-identical oscillators can also reach the phase synchronization regime, wherein a locking of the phases is produced, without a substantial associated correlation in the amplitudes [396]. Lag synchronization implies the asymptotic boundedness of the difference between the output of one system at time $t$ and the output of another shifted in time by a lag time $\tau_{\text {lag }}$ [398]. This latter phenomenon can also occur intermittently, giving rise to the intermittent lag synchronization, where the coupled systems are most of the time verifying the condition for lag synchronization, but persistent bursts of local non-synchronous behavior may intermittently affect their dynamics $[398,401]$. Analogously, imperfect phase synchronization is the regime where intermittent phase slips take place within a phase synchronization process [402]. Finally, almost synchronization is meant as the asymptotic boundedness of the difference between a subset of the variables of one system and the corresponding subset of variables of another system [403].

The natural continuation of these pioneering works was to investigate synchronization phenomena in spatially extended or infinite-dimensional systems [404-409], to test synchronization in experiments or natural systems [410-421], to study the mechanisms leading to de-synchronization [422,423], and to define unifying formal approaches that could encompass within the same framework the different synchronization phenomena [424]. A full account of the different synchronization states studied so far for chaotic systems and space-extended fields can be found in Ref. [392]. 
While in principle all those states can be observed in complex networks, historically the study of synchronization here started by dealing with oscillators whose topology of interactions showed either the scale free or the small world properties [425-430], and later it has mainly concentrated on complete synchronization phenomena of identical non linear systems, since they allowed for an analytical approach.

In this latter context, a very relevant problem is to assess conditions for the stability of the synchronous behavior for a generic network topology, and for a generic coupling configuration.

\subsection{The Master stability function approach}

We start with discussing the so called Master Stability Function approach. This method, originally introduced for arrays of coupled oscillators [431,432], has been later extended to the case of a complex networks of dynamical systems coupled with arbitrary topologies [433-437].

Let's consider a generic network of $N$ coupled dynamical units, each one of them giving rise to the evolution of a $m$-dimensional vector field $\mathbf{x}_{i}$ ruled by a local set of ordinary differential equations $\dot{\mathbf{x}}_{i}=\mathbf{F}_{i}\left(\mathbf{x}_{i}\right)$ (in fact, the technique applies to networks of time-discrete maps as well, but for the sake of clarity we will focus in the following on time continuous systems). The equation of motion reads:

$$
\dot{\mathbf{x}}_{i}=\mathbf{F}_{i}\left(\mathbf{x}_{i}\right)-\sigma \sum_{j=1}^{N} \mathscr{C}_{i j} \mathbf{H}\left(\mathbf{x}_{j}\right), \quad i=1, \ldots, N
$$

Here, $\mathbf{H}(\mathbf{x}): \mathbb{R}^{m} \rightarrow \mathbb{R}^{m}$ is a vectorial output function, $\sigma$ is the coupling strength, and $\mathscr{C}_{i j} \in \mathbb{R}$ are the elements of a zero row-sum $\left(\sum_{j} \mathscr{C}_{i j}=0 \forall i\right) N \times N$ symmetric connectivity matrix $\mathscr{C}$ with strictly positive diagonal terms $\left(\mathscr{C}_{i i}>0 \forall i\right)$, that specifies the strength and topology of the underlying connection wiring. As we will see in the following sections, the coupling matrix $\mathscr{C}$ is suitably related with the classical matrices defining the topology of the network (introduced in Sections. 2.1.6 and 2.4.1), such as the adjacency matrix $\mathscr{A}$, or the weights matrix $\mathscr{W}$ for weighted networks, or the Laplacian matrix $\Lambda$.

In order to proceed with the analytic treatment, we will make the explicit assumption that the network is made of identical systems, i.e. the evolution function $\mathbf{F}_{i}$ in Eq. (5.1) is the same for all network nodes $\left(\mathbf{F}_{i}\left(\mathbf{x}_{i}\right) \equiv \mathbf{F}\left(\mathbf{x}_{i}\right), \forall i\right)$. This assumption is crucial to ensure the existence of an invariant set $\mathbf{x}_{i}(t)=\mathbf{x}_{s}(t), \forall i$, representing the complete synchronization manifold $\mathscr{S}$, whose stability will be the object of the study. The synchronization manifold $\mathscr{S}$ is an invariant set, due to the zero row-sum condition of the coupling matrix $\mathscr{C}$ and due to the fact that the coupling function $\mathbf{H}(\mathbf{x})$ is the same for all network nodes. These two properties warrant that the coupling term vanishes exactly on $\mathscr{S}$, and therefore stability of the synchronous state reduces to take care of the system's dynamical properties along all directions in phase space that are transverse to the synchronization manifold.

For the time being, we will concentrate on the case of symmetric (thus diagonalizable) matrices $\mathscr{C}$. Let $\lambda_{i}\left[\mathbf{v}_{i}\right]$ be the set of real eigenvalues (of associated orthonormal eigenvectors), such that $\mathscr{C} \mathbf{v}_{i}=\lambda_{i} \mathbf{v}_{i}$ and $\mathbf{v}_{j}^{\mathrm{T}} \cdot \mathbf{v}_{i}=\delta_{i j}$.

The zero-row condition ensures that: (i) the spectrum is entirely semi-positive, i.e. $\lambda_{i} \geqslant 0 \forall i$; (ii) $\lambda_{1} \equiv 0$ with associated eigenvector $\mathbf{v}_{1}= \pm 1 / \sqrt{N}\{1,1, \ldots, 1\}^{\mathrm{T}}$ that entirely defines the synchronization manifold $\mathscr{S}$; and (iii) all the other eigenvalues $\lambda_{i}(i=2, \ldots, N)$ have associated eigenvectors $\mathbf{v}_{i}$ spanning all the other directions of the $m \times N$-dimensional phase space transverse to $\mathscr{S}$.

A necessary condition for stability of the synchronization manifold [392] is that the set of $(N-1) * m$ Lyapunov exponents that corresponds to phase space directions transverse to the $m$-dimensional hyperplane $\mathbf{x}_{1}=\mathbf{x}_{2}=\cdots=\mathbf{x}_{N}=\mathbf{x}_{s}$ be entirely made of negative values. Despite being a universal stability standard, it is important to warn that this condition lacks to be sufficient. Lyapunov exponents are asymptotic averages, and, as so, they account for global stability properties. However, they do not warrant that there are not unstable invariant sets in $\mathscr{S}$ itself [438], or areas of the attractor that are locally unstable [439], that can possibly produce bubbling or bursting of the dynamics away from the synchronous state (when, e.g., noise is acting). In the following, we will describe the master stability function approach in the context of Lyapunov exponents. Of course, one can start the discussion from more refined stability criteria [392], and refocus the theory for the specific chosen stability condition.

Let $\delta \mathbf{x}_{i}(t)=\mathbf{x}_{i}(t)-\mathbf{x}_{s}(t)=\left(\delta x_{i, 1}(t), \ldots, \delta x_{i, m}(t)\right)$ be the deviation of the $i$ th vector state from the synchronization manifold, and consider the $m \times N$ column vectors $\mathbf{X}=\left(\mathbf{x}_{1}, \mathbf{x}_{2}, \ldots, \mathbf{x}_{N}\right)^{\mathrm{T}}$ and $\delta \mathbf{X}=\left(\delta \mathbf{x}_{1}, \ldots, \delta \mathbf{x}_{N}\right)^{\mathrm{T}}$. 


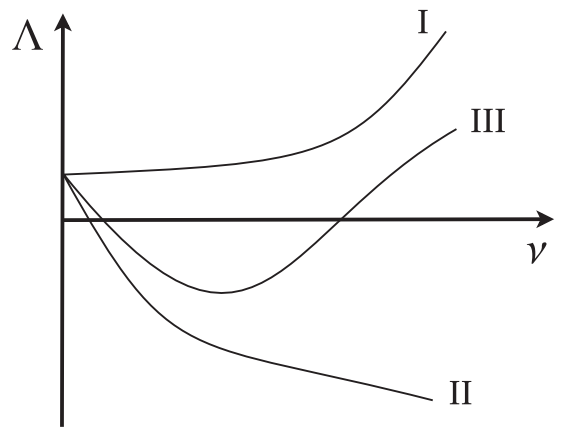

Fig. 5.1. Possible classes of master stability function for networked chaotic systems. In all cases $\Lambda(v=0)>0$ is the maximum Lyapunov exponent of the single uncoupled system. The case I (II) corresponds to a monotonically increasing (decreasing) master stability function. Case III admits a finite range of negative values for $\Lambda(v)$.

Then, one has

$$
\delta \dot{\mathbf{X}}=\left[\rrbracket_{N} \otimes \mathbf{J F}\left(\mathbf{x}_{S}\right)-\sigma \mathscr{C} \otimes \mathbf{J H}\left(\mathbf{x}_{S}\right)\right] \delta \mathbf{X},
$$

where $\otimes$ stands for the direct product between matrices, and $\mathrm{J}$ denotes the Jacobian operator.

The arbitrary state $\delta \mathbf{X}$ can be written as $\delta \mathbf{X}=\sum_{i=1}^{N} \mathbf{v}_{i} \otimes \zeta_{i}(t)\left[\zeta_{i}(t)=\left(\zeta_{1, i}, \ldots, \zeta_{m, i}\right)\right]$.

By applying $\mathbf{v}_{j}^{\mathrm{T}}$ to the left side of each term in Eq. (5.2), one finally obtains a set of $N$ variational equations for the coefficients $\zeta_{i}(t)$ that read

$$
\frac{\mathrm{d} \zeta_{j}}{\mathrm{~d} t}=\mathbf{K}_{j} \zeta_{j}
$$

where $j=1, \ldots, N$ and $\mathbf{K}_{j}=\left[\mathbf{J F}\left(\mathbf{x}_{s}\right)-\sigma \lambda_{j} \mathbf{J H}\left(\mathbf{x}_{s}\right)\right]$ is the evolution kernel.

The key point is to notice that each equation in (5.3) corresponds to a set of $m$ conditional Lyapunov exponents (the kernels $\mathbf{K}_{j}$ are calculated on $\mathscr{S}$ ) along the eigenmode corresponding to the specific eigenvalue $\lambda_{j}$. Now, $\mathscr{C}$ 's spectrum contains always the eigenvalue $\lambda_{1}=0$, whose corresponding eigenmode lies entirely within the synchronization manifold (in analogy with the properties of eigenvalues and eigenvectors of the Laplacian matrix already discussed in Section 2.1.6). The corresponding $m$ conditional Lyapunov exponents equal those of the single uncoupled system $\dot{\mathbf{x}}=\mathbf{F}(\mathbf{x})$, therefore no conditions on them will be imposed in all the remaining study.

For the sake of clarity, we will now distinguish the case of a symmetric coupling (a symmetric matrix $\mathscr{C}$ having a real spectrum) from the case of an asymmetric coupling configuration (for which, when diagonalization is allowed, the spectrum can contain also pairs of complex conjugate eigenvalues). If $\mathscr{C}$ is symmetric, all its eigenvalues are real, and they can be ordered by size as $0=\lambda_{1} \leqslant \lambda_{2} \leqslant \cdots \leqslant \lambda_{N}$. Replacing $\sigma \lambda_{i}$ by $v$ in Eq. (5.3), one obtain a parametric $m$-dimensional equation:

$$
\dot{\zeta}=\mathbf{K}_{v} \zeta=\left[\mathbf{J F}\left(\mathbf{x}_{s}\right)-v \mathbf{J H}\left(\mathbf{x}_{s}\right)\right] \zeta,
$$

from which one can extract the set of $m$ conditional Lyapunov exponents at each value of the parameter $v$. The parametrical behavior of the largest of such exponents $\Lambda(v)$ is called Master Stability Function. From what said above, the value of $\Lambda(v=0)$ will be either zero or larger than zero depending on whether $\dot{\mathbf{x}}=\mathbf{F}(\mathbf{x})$ supports a periodic or chaotic dynamics.

For $v>0$, three possible behaviors of $\Lambda(v)$ can be produced in the vicinity of the origin, defining three possible classes for the choice of the local function $\mathbf{F}(\mathbf{x})$ and of the coupling function $\mathbf{H}(\mathbf{x}):(\mathrm{I}) \Lambda(v)$ is a monotonically increasing function, (II) $\Lambda(v)$ is a monotonically decreasing function that intercepts the abscissa at some $v_{\mathrm{c}} \geqslant 0$, and (III) $\Lambda(v)$ is a $\mathrm{V}$-shaped function admitting negative values in some range $0 \leqslant v_{1}<v_{2}$. The three classes of master stability function are sketched in Fig. 5.1.

It is easy to understand that both cases (I) and (II) of Fig. 5.1 correspond to rather trivial situations. Indeed, case (I) is tantamount to say that one never stabilizes synchronization in the network for that choice of $\mathbf{F}(\mathbf{x})$ and $\mathbf{H}(\mathbf{x})$ (for all $\sigma$ values and for all possible eigenvalues' distributions, the product $\sigma \lambda_{i}$ always leads to a positive maximum 
Lyapunov exponent, and therefore the synchronization manifold $\mathscr{S}$ is always transversally unstable). The very opposite situation arises for functions $\mathbf{F}(\mathbf{x})$ and $\mathbf{H}(\mathbf{x})$ giving Master Stability curves as the one of the case II in Fig. 5.1. There, the network admits always synchronization for a large enough coupling strength, regardless on the topology of the coupling configuration (given any eigenvalue distributions it is indeed sufficient to select $\sigma>v_{\mathrm{c}} / \lambda_{2}$ (where $v_{\mathrm{c}}$ is the intersection point of the master stability function with the $v$ axis) to warrant that all transverse directions to $\mathscr{S}$ have associated negative Lyapunov exponents). In this latter case, once fixed $\dot{\mathbf{x}}=\mathbf{F}(\mathbf{x})$ and $\mathbf{H}(\mathbf{x})$ (which fix the value of $v_{\mathrm{c}}$ ) the effect of the connection topology is only to rescale (by means of $\lambda_{2}$ ) the threshold for the appearance of a synchronous state.

A non-trivial and interesting situation is case (III), which by the way corresponds to a very large class of functions $\mathbf{F}(\mathbf{x})$ and $\mathbf{H}(\mathbf{x})$ [433]. Here, $\Lambda(v)$ is negative in a finite parameter interval $\left(v_{1}, v_{2}\right)$ (with $v_{1}=0$ when $\mathbf{F}(\mathbf{x})$ supports a periodic motion). The stability condition is then satisfied for some $\sigma$ when $\lambda_{N} / \lambda_{2}<v_{2} / \nu_{1}$. The network capability to give rise to a synchronized dynamics is fully accounted for by the ratio $\lambda_{N} / \lambda_{2}$ between the largest and the second smallest eigenvalue in the spectrum of the coupling matrix: the more packed the eigenvalues of $\mathscr{C}$ are, the higher is the chance of having all Lyapunov exponents into the stability range for some $\sigma$ [433].

The situation is a bit more complicated when the coupling matrix $\mathscr{C}$ is asymmetric and diagonalizable. In such a case, the spectrum of $\mathscr{C}$ is contained in the complex plane $\left(\lambda_{1}=0 ; \lambda_{l}=\lambda_{l}^{r}+i \lambda_{l}^{i}, l=2, \ldots, N\right)$, and one has to study the parametric equation (5.4) for complex values of the parameter $v=v^{r}+i v^{i}$. An ordering of $\mathscr{C}$ 's eigenvalues can be done for increasing real parts. Gerschgorin's circle theorem $[440,441]$ asserts that $\mathscr{C}$ 's spectrum in the complex plane is fully contained within the union of circles $\left(\Gamma_{i}\right)$ having as centers the diagonal elements of $\mathscr{C}\left(d_{i}\right)$, and as radii the sums of the absolute values of the other elements in the corresponding rows $\left(\left\{\lambda_{l}\right\} \subset \cup_{i} \Gamma_{i}\left[d_{i}, \sum_{j \neq i}\left|\mathscr{C}_{i j}\right|\right]\right)$. Notice that if the coupling matrix is made of real entries, in all cases the circles have centers lying on the real axis.

If one further supposes the diagonal elements of $\mathscr{C}$ to be conveniently normalized to 1 in all possible cases, and that all non-zero off diagonal entries of $\mathscr{C}$ are negative, then relevant mathematical and physical consequences arise.

Physically, this normalization process prevents the coupling term from being arbitrarily large (or arbitrarily small) for all possible network topologies and sizes, thus making it a meaningful realization of what happens in several real world situations (such as neuronal networks) where the local influence of the environment on the dynamics does not scale with the number of connections.

Mathematically, since $\mathscr{C}$ is a zero row-sum matrix (and $d_{i}=\sum_{j \neq i}\left|\mathscr{C}_{i j}\right|$ follows from the extra assumption that all non-zero off diagonal elements are negative), this warrants in all cases and for all network sizes that $\mathscr{C}$ 's spectrum is fully contained within the unit circle centered at 1 on the real axis $\left(\left|\lambda_{l}-1\right| \leqslant 1, \forall l\right)$, giving the following inequalities: (i) $0<\lambda_{2}^{r} \leqslant \cdots \leqslant \lambda_{N}^{r} \leqslant 2$, and (ii) $\left|\lambda_{l}^{i}\right| \leqslant 1, \forall l$. This latter property is essential to provide a consistent and unique mathematical framework within which one can formally assess the relative merit of one topology against another for optimizing the propensity for synchronization of a network, regardless on the specific properties of the local dynamics.

By calling $\mathscr{R}$ the bounded region in the complex plane where the master stability function $\Lambda(v)$ provides a negative Lyapunov exponent, the stability condition for the synchronous state is that the set $\left\{\sigma \lambda_{l}, l=2, \ldots, N\right\}$ be entirely contained in $\mathscr{R}$ for a given $\sigma$. This is best accomplished for connection topologies that simultaneously make the ratio $\lambda_{N}^{r} / \lambda_{2}^{r}$ and $\mathscr{M} \equiv \max _{l} \geqslant 2\left\{\left|\lambda_{l}^{i}\right|\right\}$ as small as possible.

Master stability function arguments are currently used as a challenging framework for the study of synchronized behaviors in complex networks, especially for understanding the interplay between complexity in the overall topology and local dynamical properties of the coupled units.

At the same time, more sophisticated approaches have been developed to assess conditions for synchronization in a network of coupled units. An example is the recent connection graph stability method [437], that combines Lyapunov function approaches with graph theory, leading to the determination of a rigorous bound for the minimum coupling strength needed for yielding global synchronization. This approach allows also for describing some cases, where the coupling matrix $\mathscr{C}$ is time-dependent, i.e. $\mathscr{C}=\mathscr{C}(t)$.

Furthermore, recent studies have approached the relationships between symmetry group theory and stability of the synchronized (or patterned) solutions in networks with complex topologies [442], revealing that the symmetry groups in the architecture of complex networks can determine constraints for the appearance or stability of a given network solution.

Many relevant situations, as e.g. pulse-coupled networks of bursting neurons, correspond to inherently nonlinearly coupled systems. Therefore, the attention has also started to concentrate on nonlinearly coupled units, with the same aim 
of relating the topology of the network to the synchronization properties. For instance, when such kinds of networks are very homogeneous in the degree distribution (when all nodes have the same degree $k$ ), a recent study [443] has shown that the onset and stability of the synchronous state only depends on the number of signals each neuron is receiving, regardless on the details of the network topology. Even though such a statement strongly depends on the assumption that the degree distribution function is a delta function (and therefore it does not apply to the class of networks that are the object of the present Report), the fact that synchronization in ensembles of neurons (each one of them receiving $k$ inputs) can be ensured by means of a single condition is a relevant result.

In the following section, we will show how the application of master stability function arguments allows to prove that weighted networks provide in general enhancement of synchronization, and we will discuss some examples of weighting procedures motivated by concrete situations encountered in real networks.

\subsection{Network propensity for synchronization}

A basic assumption characterizing most of the early works on synchronization in complex networks is that the local units are symmetrically coupled with uniform undirected coupling strengths (unweighted links). As we have already discussed in Section 2.4, this simplification does not retain in general the full information on the structure of real networks. There are, indeed, paradigmatic cases where a weighting in the connections has relevant consequences in determining the network's dynamics. In ecology, for instance, the non-uniform weight in prey-predator interactions may play a crucial role in determining the food web dynamics [188,189]. In transportation networks, critical quantities that affect the global dynamics are the traffic load of a road, or the number of passengers in subways lines or airports $[30,194]$. Again, the natural differences of neurons and their dendritic connections may result in distinct capabilities of transmission and information processing in neural networks [444,445].

A great interest has born on showing how asymmetric weighted coupling configurations may enhance synchronization of complex networks. This was also motivated by the fact that asymmetry in the coupling was shown to play a fundamental role in connection with synchronization of coupled spatially extended fields [446,447].

\subsubsection{Synchronization in weighted networks: coupling matrices with real spectra}

The initial step in this direction has been recently made in Ref. [448], that considered a coupled network like the one in Eq.(5.1) where the coupling factor in the right-hand side was taken to be

$$
\frac{\sigma}{k_{i}^{\beta}} \sum_{j=1}^{N} \Lambda_{i j} \mathbf{H}\left(\mathbf{x}_{j}\right),
$$

( $\Lambda_{i j}$ being the elements of the Laplacian matrix, defined in Section 2.1.6), i.e. the connections between nodes are weighted with powers of the node degrees. In particular, Ref. [448] shows that an optimal condition $\beta=1$ for synchronization is found for a large class of complex networks.

Fig. 5.2 reports the average over 50 realizations of different networks with $N=1024$ of the quantity $R \equiv \lambda_{N} / \lambda_{2}$ as a function of the weighting parameter $\beta$, and shows that in all cases the optimal condition for synchronization (the minimum of the curve $R(\beta))$ is obtained for $\beta=1$.

By comparing Eq. (5.5) with Eq.(5.1), it is easy to realize that the condition $\beta=1$ corresponds to a situation where the input strength of the coupling is equal for all nodes (the diagonal elements of the coupling matrix $\mathscr{C}$ are normalized to 1 ), while, for $\beta \neq 1$, the diagonal elements of $\mathscr{C}$ are not normalized to 1 , and can be in general size dependent.

The relevant results of Ref. [448] is the tremendous improvement on the propensity of synchronization, obtained with a weighting procedure that retains information on the local features of the network (the node degree). Further analysis on directed and weighted networks with criteria based on the overall cost of the coupling has been carried out by the same authors in Ref. [449].

The problem of how a weighting procedure can enhance the propensity for synchronization has been studied also in the framework of properly normalized coupling schemes making use of the information contained in the overall topology [450], showing that this can give rise to further improvements in the synchronization properties. In the following we will summarize this latter approach. The idea is to scale the coupling strength between two nodes to the load of the edge connecting them, as defined in Section 2.1.2. 


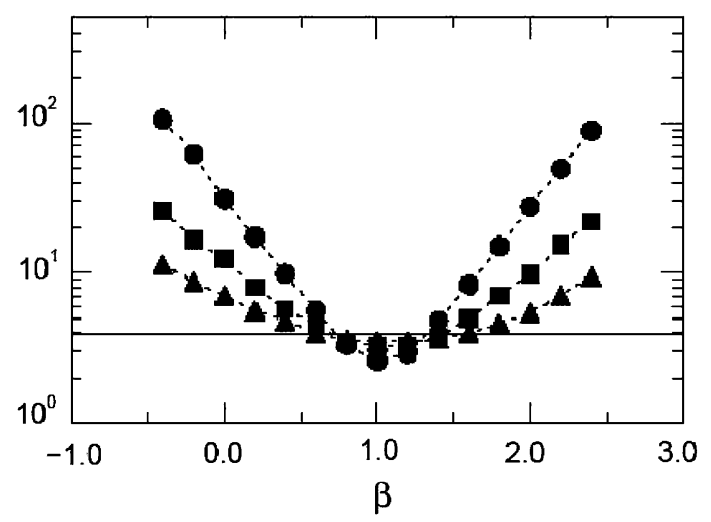

Fig. 5.2. Reprinted figure with permission from Ref. [448]. (C) 2005 by the European Physical Society. Eigenratio $R \equiv \lambda_{N} / \lambda_{2}$ vs. the weighting parameter $\beta$ in Eq. (5.5). The figure reports data coming from random networks having a scale free distribution with $\gamma=3$ (circles), $\gamma=5$ (squares), $\gamma=7$ (upper triangles), and $\gamma=\infty$ (solid line).

The load $\ell_{i j}$ of the link connecting nodes $i$ and $j$ quantifies the traffic of shortest paths that are making use of that link [42], this way reflecting a measure of the network structure at a global scale. Precisely, for each pair of nodes $i^{\prime}, j^{\prime}$ in the network, one counts the number $n\left(i^{\prime}, j^{\prime}\right)$ of shortest paths connecting them. For each one of such shortest paths, one then adds $1 / n$ to the load of each link forming it. This way, the load distribution retains full information on the network structure of pathways at a global level, since the value of each load $\ell_{i j}$ can be strongly influenced also by pairs of nodes that may be very far away from either nodes $i$ and $j$. Furthermore, it is essential to highlight that this weighting procedure is totally different from relying on the information on the node degrees, insofar as nodes with low degrees in the networks may be connected through links with very high loads, whereas nodes with high degrees may have links with very poor loads.

The equation considered in Ref. [450] reads

$$
\dot{\mathbf{x}}_{i}=\mathbf{F}\left(\mathbf{x}_{i}\right)-\frac{\sigma}{\sum_{j \in \mathcal{N}_{i}} \ell_{i j}^{\alpha}} \sum_{j \in \mathscr{N}_{i}} \ell_{i j}^{\alpha}\left[\mathbf{H}\left(\mathbf{x}_{j}\right)-\mathbf{H}\left(\mathbf{x}_{i}\right)\right]
$$

where $\alpha$ is a real tunable parameter, and $\mathscr{N}_{i}$ is the set of neighbors to the $i$ th node.

First, the coupling matrix $\mathscr{C}$ [obtained by comparing Eq. (5.6) with Eq. (5.1)] has now diagonal elements that are always normalized to 1 . Second, although $\mathscr{C}$ is asymmetric for all $\alpha$, it can be written as a product $\mathscr{C}=\mathscr{B} \mathscr{L}$, where $\mathscr{L}$ is a zero row-sum matrix with off-diagonal entries $\mathscr{L}_{i j}=-\ell_{i j}^{\alpha}$, and $\mathscr{B}=\operatorname{diag}\left\{1 /{ }_{j} \ell_{1 j}^{\alpha}, \ldots, 1 /{ }_{j} \ell_{N j}^{\alpha}\right\}$.

From matrix identities [451], $\mathscr{C}$ 's spectrum is the same as that obtained from the matrix $\mathscr{B}^{1 / 2} \mathscr{L}^{1 / 2}$, and therefore is real with non-negative values. Moreover, because $\mathscr{C}$ has a zero row-sum, the smallest eigenvalue $\lambda_{1}$ is zero, while $\lambda_{2}>0$ for connected networks, and $\lambda_{i} \leqslant 2 \forall i[134,451]$.

Other important properties of such weighting procedure concern with the various limits the coupling term can assume when changing $\alpha$. The limit $\alpha=0$ yields the best synchronizability condition of Ref. [448], being $\sum_{j \in \mathscr{N}_{i}} \ell_{i j}^{(\alpha=0)}=k_{i}$. The limit $\alpha=+\infty(\alpha=-\infty)$ induces a uni-directed $N$-link tree structure in the wiring, such that only the link with maximum (minimum) load is selected as the incoming link for each node. The resulting network will be either connected or disconnected. In the connected (disconnected) case, the ratio $\lambda_{N} / \lambda_{2}$ will be equal exactly to $2(+\infty)$, thus yielding a very strong (very weak) condition for synchronization.

By varying $\alpha$ in Eq. (5.6), Ref. [450] monitored the ratio $\lambda_{N} / \lambda_{2}$ for $\mathscr{C}$, as a suitable indicator of the propensity for synchronization for a class of scale-free networks with different degree distributions, and for random networks. Scalefree networks were obtained by the procedure introduced in Refs. $[3,146]$, and discussed in Section 2.3.5. Precisely, starting from $m+1$ all to all connected nodes, at each time step a new node is added with $m$ links, that point to old nodes with probability $p_{i}=\left(k_{i}+B\right) /\left({ }_{j}\left(k_{j}+B\right)\right)$. While all details of this model are discussed in Section 2.3.5, here we recall some of its properties that play an important role in the context of determining the propensity for synchronization of the network. 

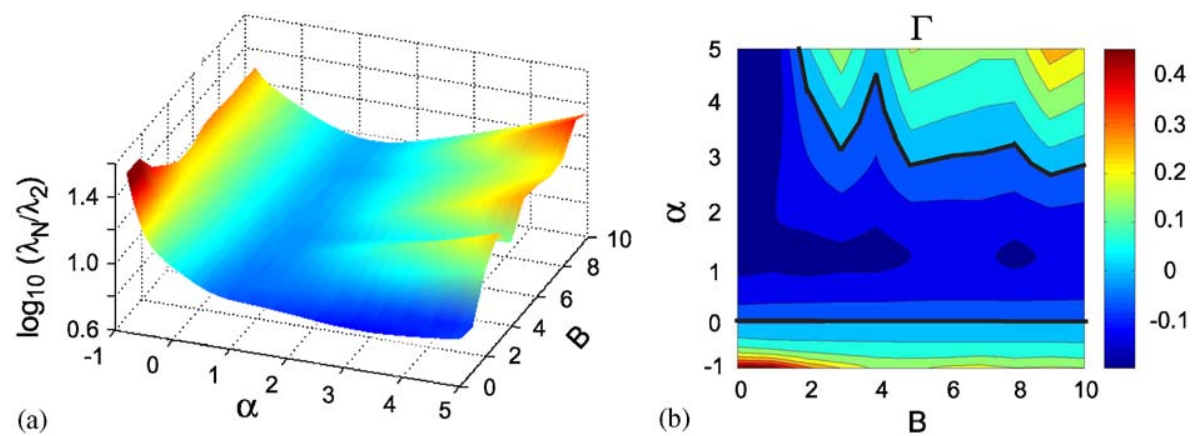

Fig. 5.3. Reprinted figure with permission from Ref. [450]. (C) 2005 by the American Physical Society. (a) $\lambda_{N} / \lambda_{2}$ (in logarithmic scale) for SF networks vs. the parameter space $(\alpha, B)$. (b) $\Gamma$ (see text for definition) vs. $(\alpha, B)$. In all cases $m=2$, and the reported values refer to an average over 10 realizations of networks with $N=1000$ nodes. The domain with $\Gamma<0$ is outlined by the black contours drawn on the figure.

$B$ is a tunable real parameter, representing the initial attractiveness of each node. The $\gamma$ exponent of the power law scaling in the degree distribution $\left(p(k) \sim k^{-\gamma(B, m)}\right)$ is selected to be $\gamma(B, m)=3+B / m$ in the thermodynamic $(N \rightarrow \infty)$ limit. The average degree is by construction $\langle k\rangle=2 m$ (thus independent on $B$ ), but the heterogeneity of the degree distribution can be strongly modified by $B$.

The class of networks with high homogeneous degree distribution is represented by random networks obtained via the rewiring procedure proposed in Ref. [28], and does correspond to fix $p=1$ in the model of Watts and Strogatz described in Section 2.3.3.

Fig. 5.3 (a) shows the logarithm of $\lambda_{N} / \lambda_{2}$ in the parameter space $(\alpha, B)$ for scale free networks. It is important to notice that the surface of $\lambda_{N} / \lambda_{2}$ has a pronounced minimum at $0<\tilde{\alpha} \simeq 1$ for all values of $B$ above a given $B_{\mathrm{c}}>0$. Because here $\alpha=0$ recovers the optimal condition when only the information on node degrees is used (the condition $\beta=1$ in Ref. [448]), this indicates that a weighting procedure based on the link loads always enhances the propensity for synchronization. This is quantified in Fig. $5.3(\mathrm{~b})$, where the quantity $\Gamma=\log \left(\lambda_{N} / \lambda_{2}\right)-\left[\log \left(\lambda_{N} / \lambda_{2}\right)\right]_{\mid} \alpha=0$ is shown in the parameter space. The large region of negative values of $\Gamma$ corresponds to topological structures $(B)$ and weighting configurations $(\alpha)$ providing better synchronization propensities than a weighting process based on the node degrees.

This represents a remarkable result, insofar as it indicates that conveying the global complex structure of shortest paths into a weighting procedure gives in that range a better criterion for synchronization than solely relying on the local information around a node. When increasing $\alpha$, the region of enhancement is limited for $B>B_{\mathrm{c}}$ [as indicated by the upper black line in Fig. 5.3 (b)]. Increasing $\alpha$ above $\tilde{\alpha}$, indeed, introduces two effects: the first is that it makes it more likely that a unidirected tree structure appears in the network, and the second is that it increases the chance of disconnecting the network. These two effects are competing to determine synchronous behavior, insofar as the former increases the likelihood of synchronization only if the network remains connected. Therefore, a second critical value $\alpha_{\mathrm{c}}(B)>\tilde{\alpha}$ can be expected such that the disconnection mechanism dominates the tree structure induction if $B>B_{\mathrm{c}}$.

While Fig. 5.3 focus on the case $m=2$, increasing $m$ diminishes the value of $\tilde{\alpha}$ (thus gradually reducing the enhancement of global vs. local weighting procedure), and it increases the likelihood of disconnecting the network in the limit $\alpha \rightarrow \infty$, thus inducing $B_{\mathrm{c}} \rightarrow 0$. Instead, $m=1$ always ensures that the network remains connected at $\alpha \rightarrow \infty$, regardless of $B$.

The situation with random networks is illustrated in Fig. 5.4: Fig. 5.4(a) reports the behavior of $\log \left(\lambda_{N} / \lambda_{2}\right)$ vs. $\alpha$, indicating that here also the weighting procedure based on link loads enhances the propensity for synchronization of the network (the minimum of the curve is always positioned at $0<\tilde{\alpha} \simeq 1$ ); while Fig. 5.4(b) reports the comparative quantity $\Gamma_{\mathrm{c}}=\left[\log \left(\lambda_{N} / \lambda_{2}\right)\right]_{\text {SCALE FREE }}-\left[\log \left(\lambda_{N} / \lambda_{2}\right)\right]_{\text {RANDOM }}$ in the parameter space $(\alpha, B)$, indicating that for all $\alpha>0$ weighted scale free configurations provide better topologies for inducing a synchronized behavior than random networks.

\subsubsection{Synchronization in weighted networks: coupling matrices with complex spectra}

Both the approaches developed in Refs. [448,450] delt with situations wherein the coupling matrix $\mathscr{C}$ had a real spectrum of eigenvalues. Motivated by what happens in social networks $[452,453]$ (where interactions between individuals 

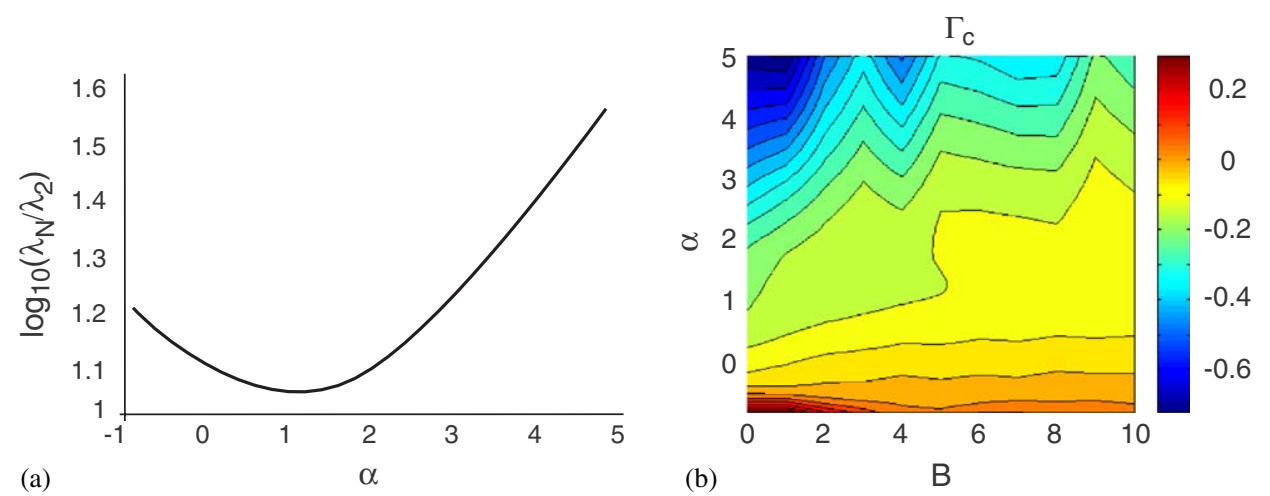

Fig. 5.4. Reprinted figure with permission from Ref. [450]. (C) 2005 by the American Physical Society. (a) $\lambda_{N} / \lambda_{2}$ (in logarithmic scale) for random graphs vs. $\alpha$. (b) $\Gamma_{\mathrm{c}}$ (see text for definition) vs. the parameter space $(\alpha, B)$. Random networks have $\langle k\rangle=2 m=4$, identical to that of scale free networks. In the domain where $\Gamma_{\mathrm{c}}<0$ scale free networks synchronize better than random networks. Same conditions as in the caption of Fig. 5.3 .

are not symmetric, and depend upon several social factors, such as age, social class or influence, personal leadership or charisma), Ref. [454] analyzed networks of asymmetrically coupled dynamical units, where asymmetry is explicitly related to an age order among different nodes. In practice, the idea is that the direction of an edge can be determined by an age ordering between the connected nodes. For instance, in growing networks, such age ordering will be naturally related to the appearance order of the node during the growing process.

This is reflected by a zero row-sum coupling matrix $\mathscr{C}_{i j}$ in Eq. (5.1) with off diagonal entries:

$$
\mathscr{C}_{i j}=-\mathscr{A}_{i j} \frac{\Theta_{i j}}{\sum_{j \in \mathscr{N}_{i}} \Theta_{i j}},
$$

where $\mathscr{A}$ is the adjacency matrix, and $\Theta_{i j}=(1-\theta) / 2\left(\Theta_{i j}=(1+\theta) / 2\right)$ for $i>j(i<j)$. Following the notations of Section $2.1, \mathscr{N}_{i}$ is the set of $k_{i}$ neighbors of the $i$ th node.

The parameter $-1<\theta<1$ governs the coupling asymmetry in the network. Precisely, $\theta=0$ yields the optimal synchronization condition of Ref. [448], being $\sum_{j \in \mathscr{N}_{i}} 1=k_{i}$, while the limit $\theta \rightarrow-1(\theta \rightarrow+1)$ gives a unidirectional coupling configuration wherein the older (younger) nodes drive the younger (older) ones.

Though being asymmetric, for $\theta=0$ the matrix $\mathscr{C}$ has a real spectrum of eigenvalues, and the results are the same as those obtained in Ref. [448] for $\beta=1$. Conversely, for a generic $\theta \neq 0$, the coupling matrix has a spectrum contained in the complex plane $\left(\lambda_{1}=0 ; \lambda_{l}=\lambda_{l}^{r}+j \lambda_{l}^{i}, l=2, \ldots, N\right)$. Furthermore, by construction, the diagonal elements of $\mathscr{C}$ are normalized to 1 in all possible cases. The physical and mathematical consequences of these stipulations are discussed in Section 5.2.

As discussed above, if $\mathscr{R}$ is the bounded region in the complex plane where the master stability function provides negative Lyapunov exponents, the stability condition for the synchronous state is that the set $\left\{\sigma \lambda_{l}, l=2, \ldots, N\right\}$ be entirely contained in $\mathscr{R}$ for a given $\sigma$, which is best realized when both the ratio $\lambda_{N}^{r} / \lambda_{2}^{r}$ and $\mathscr{M} \equiv \max _{l}\left\{\left|\lambda_{l}^{i}\right|\right\}$ are simultaneously made as small as possible.

With this in mind, Ref. [454] analyzed the effects of heterogeneity in the node degree distribution, by comparing the propensity for synchronization of the class of scale free networks introduced in the previous section with that of a highly homogeneous Erdös-Rényi random network [115] (see Section 2.3.1), having connection probability $P=2 m /(N-1)$ (giving the same average degree $\langle k\rangle=2 \mathrm{~m}$ ), and an arbitrary initial age ordering.

Fig. 5.5a reports $\lambda_{N}^{r}$ and $\lambda_{2}^{r}$ vs. $\theta$ for scale free networks with $m=5$ and $B=0$ (solid line) and for the selected random graph (dashed line). These calculations were performed by averaging over 24 different realizations of networks with 500 nodes. It is possible to notice that, for the random network, the curve $\lambda_{N}^{r}(\theta)\left[\lambda_{2}^{r}(\theta)\right]$ displays a minimum [a maximum] for $\theta=0$, showing that asymmetry here deteriorates the network propensity for synchronization, if compared with the optimal condition of Ref. [448] based on the information on the node degree. At variance, for scale free, the difference between $\lambda_{N}^{r}$ and $\lambda_{2}^{r}$ continuously shrinks, as $\theta$ decreases. Fig. $5.5 \mathrm{~b}$ reports the behavior of the eigenratio $\lambda_{N}^{r} / \lambda_{2}^{r}$, making it clear that while the best synchronizability condition for random networks is $\theta=0$, scale free networks show 


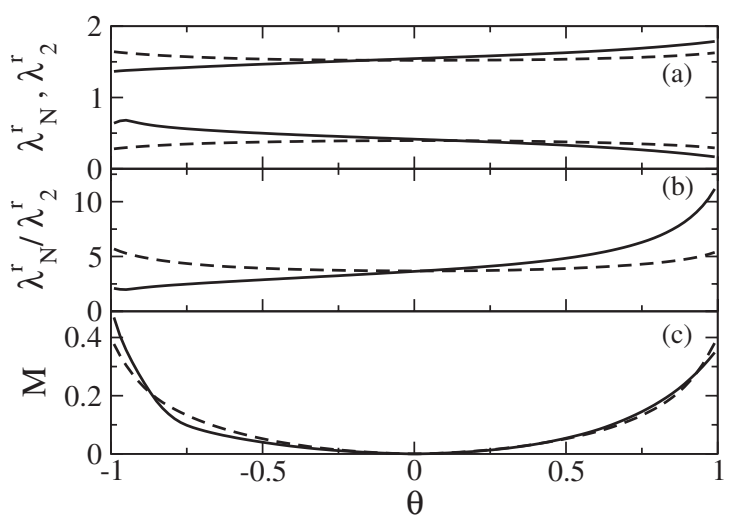

Fig. 5.5. Reprinted with permission from Ref. [454]. (C) 2005 by the American Physical Society. $\lambda_{N}^{r}$ and $\lambda_{2}^{r}$ (a), $\lambda_{N}^{r} / \lambda_{2}^{r}$ (b), and $\mathscr{M}$ (c) vs. $\theta$ for scale free ( $m=5$ and $B=0$, solid lines) and random networks (dashed lines).
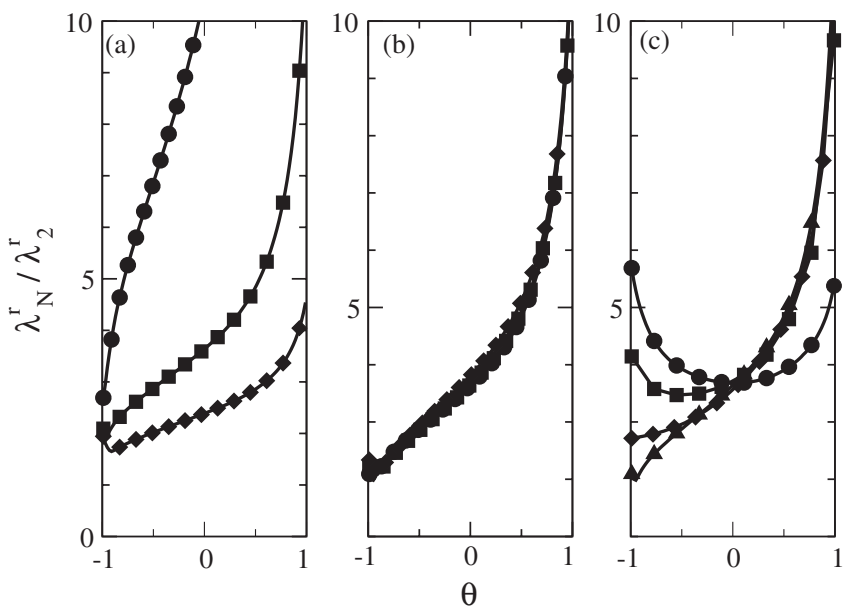

Fig. 5.6. Reprinted figure with permission from Ref. [454]. (C) 2005 by the American Physical Society. $\lambda_{N}^{r} / \lambda_{2}^{r}$ vs. $\theta$ for (a) scale free networks with $B=0$ and $m=2$ (circles), 5 (squares), 10 (diamonds); (b) scale free networks with $m=5$ and $B=0$ (circles), 5 (squares), and 10 (diamonds); (c) Erdös-Rényi random network with arbitrary age order (circles), Erdös-Rényi random network with age depending on degree (squares), scale free networks with $m=5$ and $B=0$ without connection between nodes 1 and 5 (diamonds), and scale free networks with $m=5$ and $B=0$ (triangles).

a better (worse) propensity for synchronization for $\theta \rightarrow-1(\theta \rightarrow 1)$. The imaginary part of the spectra is illustrated in Fig. 5.5c, that reports $M$ vs. $\theta$, and that indicates that only very small differences exist between the scale free and the random network configurations in the whole range of the asymmetry parameter. This fact highlights that the contribution to network synchronizability of the imaginary part of the spectra does not depend significantly on the specific network structure.

Ref. [454] also investigated the propensity for synchronization in scale free networks at different $m$ and $B$ values. In Fig. 5.6a, the propensity for synchronization is compared for different $m$ at $B=0$. As $m$ increases, so does the average connectivity, and synchronizability is naturally enhanced. An important point is that the monotonically decreasing behavior of $\lambda_{N}^{r} / \lambda_{2}^{r}$ with $\theta$ persists for all $m$, indicating that synchronization is always enhanced in growing scale free networks when $\theta$ becomes smaller. In Fig. 5.6(b), the propensity for synchronization is compared at $m=5$ and for various values of $B$, altering the exponent of the degree distribution. For all $B$ values, the enhancement for negative $\theta$ holds.

This leads us to discuss a very important point: which are the essential topological ingredients enhancing synchronization in weighted networks? The answer is that only the simultaneous action of many ingredients provide the best conditions for synchronization. 
The first ingredient is that the weighting must induce a dominant interaction from hub to non-hub nodes. This can be easily understood by a simple example: the case of a star network consisting of a single large hub (the center of the star) and several non-hub nodes connected to the hub. When the dominant coupling direction is from the non-hub nodes to the hub node, synchronization is impossible because the hub receives a set of independent inputs from the different non-hub nodes. In the reverse case (when the center drives the periphery of the star) synchronization can be easily achieved. The very same mechanism occurs in the case of the age order. Indeed, for positive (negative) $\theta$ values, the dominant coupling direction is from younger (older) to older (younger) nodes. Now, in growing scale free networks the minimal degree of a node is by construction $m$ and older nodes are more likely to display larger degrees than younger ones, so that a negative $\theta$ here induces a dominant coupling direction from hubs to non-hub nodes.

A second fundamental ingredient is that the network contains a structure of connected hubs influencing the other nodes. This is in general accounted for by a proper normalization in the off diagonal elements of $\mathscr{C}$, assuring that hubs receive an input from a connected node scaling with the inverse of their degree, and therefore the structure of hubs is connected always with the rest of the network in a way that is independent on the network size. For age ordered growing scale free networks, the non-zero off diagonal elements of the coupling matrix are given by:

$$
\mathscr{C}_{i j}=\frac{1 \mp \theta}{k_{i}-\theta\left[k_{i}-2 \min (i-1, m)\right]},
$$

where $\mp$ stands for $i>j$ and $i<j$, respectively.

The validity of what discussed above can be made evident by performing measurements of the propensity for synchronization over a series of ad hoc modified networks. The results are summarized in Fig. 5.6c. First, one can reorder the node age in the Erdös-Rényi random network according to each node degree. The resulting $\lambda_{N}^{r} / \lambda_{2}^{r}(\theta)$ (curve with squares) shows now a minimum for an asymmetric configuration $(\theta \approx-0.5)$, in contrast to the case with arbitrary aging (curve with circles). This confirms the need of a dominant interaction from hubs to non-hubs for improving synchronization, also for highly homogeneous networks. As for the second ingredient, starting from a scale free network with $m=5$ and $B=0$ (curve with triangles), one can artificially disconnect the initially existing link between the two hubs with highest degree in the network (the first and fifth nodes in the realization referred to by Fig. 5.6c). The result is shown in the curve with diamonds, where one sees that such a tiny perturbation (the difference in the two networks is limited to only a link) is already sufficient to substantially weaken the network propensity for synchronization. The situation remains however better than the two random network cases, indicating that the structure of growing aged network inherently enhances synchronization.

The examples of Refs. $[448,450,454]$ have undoubtedly shown that weighted networks is the most promising framework for the study of how the architectural properties of the connection wiring influence the efficiency and robustness of the networked system in giving rise to a synchronized behavior. It has to be expected, therefore, that much effort will be stimulated in the forthcoming years to investigate ad hoc weighted configurations, for assessing more deeply the underlying mechanisms for the formation of collective dynamics in all those circumstances where the weighting features can be directly extracted from available data.

At the end of this section it has to be mentioned that recently some works have dealt with the case of time varying connection matrices $\mathscr{C}$. In particular, the limit of blinking networks $[455,456]$ has been recently considered, where the wiring of connections is rapidly (i.e. with a characteristic time scale much shorter than that of the networked system's dynamics) switching among different configurations. In these conditions, it has been found that synchronous motion can be established for sufficiently rapid switching times even in the case in which each visited wiring configuration (if taken as fixed) would prevent synchronization.

\subsection{Synchronization of coupled oscillators}

After having discussed the general stability properties of synchronization states in complex networks, we review the most significant obtained results in the study of synchronization of different networked systems. We begin with considering networks of coupled oscillators. This investigation, indeed, started soon after the first network models were proposed, particularly with respect to limit cycle oscillators following the Kuramoto dynamics, that allow for both analytical and fast numerical simulations approaches.

The first works on conditions and effects of synchronization in complex networks were reported by Watts [5] and Barahona and Pecora [433]. Watts studied numerically the Kuramoto model on top of WS small-world networks, while 
Barahora and Pecora addressed analytically the conditions for complete synchronization of chaotic systems on different kinds of graphs, including WS small-world networks. In what follows, we concentrate on summarizing the case of scale-free networks.

There are several relevant situations, for instance in biology, where it is useful to consider the nodes of a given network as oscillatory systems. Examples are ensembles of coupled and pulse-coupled oscillators with and without time delay, widely used because of their relevance to natural systems such as chirping crickets and flashing fireflies, among others [457,458]. For such a reason, let us consider a generic graph where each node $i(i=1, \ldots, N)$ is a planar rotor characterized by an angular phase, $\theta_{i}$, and a natural or intrinsic frequency $\omega_{i}$. Two oscillators interact if they are connected by an edge of the underlying graph. The individual dynamics of the $i$ th node is described by:

$$
\dot{\theta}_{i}=\omega_{i}+\sigma \sum_{j \in \mathcal{N}_{i}} \sin \left(\theta_{j}-\theta_{i}\right),
$$

where $\mathscr{N}_{i}$ is the set of neighbors of $i$, and $\sigma$ is the coupling strength, identical for all edges. The set of natural frequencies and the initial values of $\theta_{i}$ are in general randomly chosen from a given distribution $[459,460]$.

The original Kuramoto model corresponds to the simplest case of globally coupled (complete graph) equally weighted oscillators, where the coupling strength is taken to be $\sigma=\varepsilon / N$ in order to warrant the smoothness of the model behavior also in the thermodynamic limit $N \rightarrow \infty[459,460]$. In this case, the onset of synchronization occurs at a critical value of the coupling strength $\varepsilon_{\mathrm{c}}=2 / \pi g\left(\omega_{0}\right)$, where $g(\omega)$ is the distribution from which the natural frequencies are drawn, and $\omega_{0}$ represents the mean frequency of the ensemble. The transition to synchronization is a second-order phase transition characterized by the order parameter

$$
r(t)=\left|\frac{1}{N} \sum_{j=1}^{N} \mathrm{e}^{\mathrm{i} \theta_{j}(t)}\right| .
$$

When the two limits $N \rightarrow \infty$ and $t \rightarrow \infty$ are considered (and for $\varepsilon \geqslant \varepsilon_{\mathrm{c}}$ ), the order parameter $r$ behaves as $r \sim\left(\varepsilon-\varepsilon_{\mathrm{c}}\right)^{\beta}$, with $\beta=1 / 2$.

The mechanism through which synchronization emerges in the system is as follows. As the coupling takes very small values, the strength of the interactions is not enough to break the incoherence produced by the individual dynamics of each oscillator. When a critical value $\sigma_{\mathrm{c}}$ is overcome, some elements lock their relative phases and a cluster of synchronized nodes comes up. When the coupling is further increased, the population of oscillators is split into a partially synchronized state made up of oscillators locked in phase (that adds to $r$ ), and a group of nodes whose natural frequencies are too spread as to be part of the coherent pack. Eventually, after further increasing in $\sigma$, more and more nodes get entrained around the mean phase, and the system settles in a completely synchronous state (where $r \approx 1$ ). The globally coupled Kuramoto model has been extensively studied during the last several years, and a detailed and recent review on this subject can be found in Ref. [461].

With the growing interest in complex networks, several groups have moved the attention to investigate the synchronization phenomena of the Kuramoto model in complex wirings. Refs. [462,463] have studied numerically the conditions for the onset of synchronization in random scale-free networks. Specifically, they studied the Kuramoto model on top of Barabási-Albert networks and on top of small structures (motifs) that were relevant in different biological and social networks, with the aim of inspecting the critical point associated to the onset of synchronization, i.e., when small groups of synchronized oscillators first appear in the system.

They reported that the onset of synchronization for BA networks occurs at a small, though non-zero, value of the coupling strength, with a critical exponent around 0.5. They found that the critical point does not depend on the size of the system $N$, in sharp contrast with the all-to-all coupling configuration. Moreover, as it was discovered later on, the choice of the order parameter seems to be a crucial point when analyzing the conditions for the existence of the transition threshold. In Refs. [462,463], Eq. (5.10) was used for the definition of the order parameter.

Soon afterwards, several other authors [464-466] have investigated the same problem from a theoretical perspective, as well as with numerical simulations. The results do not fully clarify whether or not the critical point exists. The main difficulty comes from the fact that there is no a unique consensus about the set of differential equations describing the system dynamics, and what the order parameter should be. Lee reported the lack of a critical point in power law graphs when $2<\gamma<3$, which is recovered when the second moment of the distribution converges (that is for $\gamma>3$ ). No estimates can be done for $\gamma=3$ since there the relevant parameters of the system diverge. 
The same qualitative behavior was reported in Refs. [465,466], where different analytical approaches were introduced. The mean field theory $[464,465]$ predicts that the critical point is determined by the all-to-all Kuramoto value, $\sigma_{0}$, rescaled by the ratio between the first two moments of the degree distribution, $\sigma_{m f}=\sigma_{0}\langle k\rangle /\left\langle k^{2}\right\rangle$.

Restrepo et al. [466] extended the results of Refs. [464,465] by generalizing the mean-field approach. Their results, supported by numerical simulations, show that the coupling strength at which the transition occurs is determined by the largest eigenvalue of the adjacency matrix. Moreover, they also point out that the mean-field approximation fails to predict the critical coupling strengths when the heterogeneity of the network increases (as, e.g., when $\gamma$ gets closer or below to 3), because of the effect of the few nodes with a high degree (hubs) that are not properly taken into account in the mean field theory. At the same time, their time-averaged-theory seems to reproduce better the numerical estimates.

It is worth noticing that Refs. [462,463] have used the classical order parameter of Eq. (5.10), while the analysis of Refs. [464-466] made use of a rescaled parameter, given by

$$
r(t)=\left|\frac{1}{\sum_{l} k_{l}} \sum_{j=1}^{N} k_{l} \mathrm{e}^{\mathrm{i} \theta_{j}(t)}\right| .
$$

This difference may be at the root of the apparent contradiction in the results for $\gamma$ close to 3. In principle, there are reasons for the use of either one of the two parameters. Eq. (5.10) does not assign weights to the different connectivity classes, while Eq. (5.11) incorporates in the definition of the order parameter the connectivity of each node, which at first glance seems to be reasonable. However, this later assumption may have the effect of partially destroying the inherent heterogeneity of the underlying network by somehow homogenizing the contribution of each node to the synchronous state. Clearly, this issue needs to be addressed in more details in future works.

The other point of debate is whether or not one should weight the coupling strength entering Eq. (6.1), following the spirit of the previous section. For instance, Lee introduced a coupling strength rescaled by $\langle k\rangle$, i.e., $\sigma /\langle k\rangle$. While this is a constant factor for a given system size, it does not capture the main topological feature of scale-free networks: the fluctuations of the connectivity distribution. Thus one may ask whether dividing by $\langle k\rangle$ produces the same effects as normalizing by any other constant.

Finally, we briefly refers to clustering and modular synchronization of oscillator networks. It is believed that, as the route to complete synchronization is made up of groups of synchronized oscillators that grow and coalesce, one would be able to detect structural properties of the underlying network by just a fine tuning in the coupling strength. Oh et al. [467] studied the Kuramoto model on two different types of modular complex networks, finding that the synchronization transition crucially depends on the type of inter-modular connections. MacGraw and Menzinger [468], also studied the effects of clustering. Moreno et al. [462] have shown that the relaxation time $\langle\tau\rangle$ for synchronization of hubs is shorter than that of less connected nodes. In particular, for BA networks $\langle\tau\rangle \sim k^{-1}$. Hence, the more connected a node is, the more stable it is. Such power-law behavior points to an interesting result, namely, it is easier for an element with high $k$ to get locked in phase with its neighbors than for a node linked to just a few others. Furthermore, the destabilization of a hub does not destroy the synchrony of the group it belongs to. On the contrary, the group formed by the hub's neighbors recruits it again. All these results indicate that it may be possible to use synchronization phenomena to unravel highly clustered structures embedded within the wiring of a complex network.

\subsection{Synchronization of chaotic dynamics}

At the beginning of this chapter we have largely discussed the master stability approach for the assessment of the synchronizability of a given network. Here, we review several other approaches that have been used to investigate synchronization in complex networks.

In pioneering works, the value of the coupling strength necessary to synchronize a network of arbitrarily coupled identical systems was studied [469-471]. This value was found to be ruled by the structure of the coupling matrix and by the coupling strength necessary to synchronize two elements of the network. The so-called Wu-Chua conjecture states that the coupling threshold for synchronization in a given array of $n$ linearly coupled oscillators is

$$
\sigma_{t h}=\frac{2 \sigma_{2}^{\prime}}{\left|\lambda_{2}\right|}
$$


where $\sigma_{2}$ is the synchronization threshold for synchronization of two oscillators, and $\lambda_{2}$ is the smallest non-null eigenvalue of the Laplacian matrix already discussed in Section 2.1.6.

This conjecture, on its turn, implies that a network of diffusively coupled identical oscillators can always be synchronized for sufficiently high coupling strengths [469-471]. Results from the analysis of networks formed by coupled Lorenz or Chua's oscillators confirmed the close relationship between the value of $\lambda_{2}$ and the global synchronization of the network.

We must notice that this approach is valid under the assumption that when the transversal mode associated to the eigenvalue $\lambda_{2}$ is stable, all the others transversal modes are also stable. As largely discussed in the previous Section 5.2, this assumption is valid only for those oscillators whose evolution function gives a Master Stability Function of class II in Fig. 5.1, whereas many relevant cases exist where the coupled oscillators display a de-synchronization bifurcation when the coupling strength is increased (as, for instance, the $x$-coupled Rössler oscillators) [472-474].

The propensity for synchronization (generally called synchronizability) produced by complex topologies has been largely studied in recent works [425,433,475-481]. Although some of these studies present slight differences in the assessment of the propensity for synchronization, all of them agree with the main results of Section 5.3 that the ability of a given network to synchronize is strongly ruled by the structure of connections.

In particular, some studies suggested that small-world wirings always lead to enhance synchronization as compared with regular topologies [426,427,433,475,478]. Initially, this enhancement was attributed to the decreasing of the smaller average network distance between nodes. In fact, the statement that synchronization can be enhanced by the addition of any amount of random shortcuts is not always true [431,473]. The addition of arbitrary shortcuts to a locally regular coupled array may, under certain circumstances, result in a destabilization of the synchronized state [482]. Moreover, synchronization can be affected by other quantities such as the degree distribution, characteristic path length, and betweenness centrality [425,433,481], and it was manifested that synchrony can be even deteriorated when increasing the heterogeneity in the connectivity distribution at the same average network distance [425].

A recent numerical study of a SW network of Rössler oscillators revealed that phase synchronization may arise in the small-world regime. Phase locking was found to depend on the coupling strength, the array size, or the distribution of the modes (natural frequencies) of each oscillator, as well as on the amount of random shortcuts in the connections [483].

The small-world wiring seems therefore to yield an enhancement of synchronization (both in phase and amplitude), but nevertheless the relationship between the emergence of the SW regime (based on topological properties) and the arising of collective behaviors remains unclear. An example is the fact that the synchronization threshold does not coincide (neither in its value nor in its scaling properties) with that for the onset of the SW behavior [433].

Synchronization of chaotic flows [476,484] or phase synchronization of chaotic maps [485] on top of scale-free networks was also demonstrated. Furthermore, networks and arrays of coupled maps were studied in the case in which the connections between units involve a time delay, to investigate the synchronization properties as a function of the underlying graph topology [486], or when the time delays among units are randomly selected [487].

We have already discussed in Chapter 3 the fact that complex wirings may display an intrinsical robustness again structural damage [275]. In scale free networks, for instance, the clustered organization of nodes as well as the connectivity patterns between such clusters, render the network structure tolerant to random removal of nodes but very vulnerable to targeted attacks [275]. This vulnerability not only affects static processes, but it is also directly related to the robustness of any collective dynamics emerging in such networks. Numerical simulations, for instance, have revealed that synchronization processes remain almost unchanged in scale free networks when a 5\% of the total of nodes are randomly removed, while the network splits into isolated sub-networks (thus destroying synchronization) if only $1 \%$ of target (highly connected) nodes are removed [478,484].

Finally, we briefly discuss the studies on the desynchronization mechanisms for networks. As far as coupled chaotic systems are considered, there exist many different scenarios by which synchronization is lost [392]. For instance, a counterintuitive desynchronization scenario is characterized by a destabilization of the synchronized state produced by an increase of the coupling strength [431,472]. Known as short-wavelength bifurcation, this phenomenon is often observed in diffusively coupled arrays of chaotic oscillators, and is characterized by an excitation of the shortest spatial wavelength with an increasing of the coupling, which induces the desynchronization. A direct consequence of this phenomenon is the existence of an upper limit in the number of chaotic oscillators that can be synchronized, upon which the synchronized state of a given network becomes unstable $[472,473]$. 
In a recent related work, another desynchronization mechanism has also been studied in networks with arbitrary topology. Known as bubbling, this phenomenon arises when the synchronized state of non-identical systems is sporadically interrupted, yielding bursts of desynchronized motions [438]. The spatial patterns produced by these bursts were found to be ruled not only by the local dynamics of each node, but also by the structure of the coupling matrix [474].

\subsection{Other collective behaviors in networks of ordinary differential equations}

We finally review the other collective behaviors that have been studied in networks of ordinary differential equations (NODEs). NODEs of different kinds have, indeed, been largely used to model collective dynamics as diverse as those observed in Josephson junction arrays, chemical reactions, ecological systems, and networks of both physical (lasers, electronic circuits) and biological (neurons, cardiac cells) systems [488]. Furthermore NODEs models have been used to describe non-trivial collective phenomena as self-organization, travelling waves, defect propagation, synchronization and spatio-temporal chaos [392].

In the study of interacting oscillators, three types of coupling schemes are currently considered: global coupling where each unit interacts with all the others, local coupling where an element interacts with its neighbors (defined by a given metric), and non-local or intermediate couplings. In an ensemble of globally coupled oscillators, the structure of connections is not dependent on the spatial distance between the oscillators. In such networks, each individual unit is influenced by the global dynamics through an interaction of a mean-field type. In networks of both limit-cycle and chaotic oscillators with slightly different oscillation modes, a phase transition associated to a collective and coherent behavior was produced by an increasing of the coupling strength [459,489-491].

However, a global coupling scheme may present some disadvantages when the units are embedded in a physical array such that the space dimension is relevant for the coupling structure. In some real population of oscillators as neural networks or power supply networks, long range connections (necessary to the all-to-all interactions) may imply a high cost in terms of energy (see Section 2.5 for the details of the topology constraints that may arise in geographically embedded networks).

In an opposite approach, several studies considered oscillators embedded in $D$-dimensional lattices, where each unit interacts only with its nearest neighbors. However, this approach presents severe theoretical difficulties for its analysis $[460,492,493]$. Although different collective regimes can be observed (global or partial synchronization, anti-phase synchronization, phase clustering, coherence resonance) in large ensembles of coupled chaotic or periodic elements, cooperative dynamics are strongly dependent on the size and dimension of the lattices, as well as on the distribution of the eigenfrequencies of each oscillator [494-501].

Inspired from some biological systems, where the cell-to-cell interaction is mediated by rapidly diffusive chemical transmitters, some non-local coupling schemes have been proposed to take into account the natural decay of the information content with the distance. In both theoretical and experimental studies, the dependence of the spatial correlation on the range of non-local coupling was found to decay with a power law [502-505]. Other long-range interaction schemes include the power-law couplings, in which the interaction strength decays with the lattice distance following a power law $r^{-\alpha}$ where $r$ is the distance between the oscillators and $\alpha \geqslant 0$ defines the range of the coupling: the limit $\alpha \rightarrow 0$ corresponds to the nearest neighbor coupling, whereas an uniform coupling is obtained for $\alpha=0$. In large networks of limit-cycle or chaotic oscillators, power law coupling schemes were found to yield collective behaviors which also depend on the range of coupling (given by the parameter $\alpha$ ) and the distribution of the modes (natural frequencies) of each unit [506-509].

In real physical systems consisting of interacting units, a certain degree of randomness may exist not only in the intrinsic properties of each unit, but also in the connections between them. Several studies have shown that the former natural disorder (mismatch of parameters) may strongly affect the collective behaviors in both global or local coupling networks. Hence, the emergence of new features in the cooperative dynamics may be expected for disordered connections.

In statistical mechanics, random interactions in magnetic systems are known to produce ordered phase regimes. Known as spin glasses, these ordered states are characterized by a vanishing overall magnetization. Interestingly, the properties of these states and the corresponding phase transitions are very different from the phases observed in regular magnets [510]. 
Most of the studies about NODEs with random interactions considered coupled phase oscillators of the kind [459]:

$$
\dot{\phi}_{i}(t)=\omega_{i}-\sigma \sum_{j=1}^{N} \mathscr{C}_{i j} \sin \left[\phi_{j}-\phi_{i}\right], \quad i=1, \ldots, N,
$$

where $\phi_{i}$ denotes the phase of the $i$ th oscillator, and $\omega_{i}$ its natural frequency.

In a pioneering work [511], phase locking in an lattice array was studied for different topologies: regular nearest neighbors coupling, random-local coupling (where the weights of connections $\mathscr{C}_{i j}$ were randomly drawn from a Gaussian function related to the distance to its nearest neighbors), and long-range sparse connections (where the probability of connecting a node to a given number of neighbors was ruled by a Gaussian function centered on the current node, which yielded an asymmetric matrix $\mathscr{C}_{i j}$ whose entries took values of 1 with a given probability $p$ and zero otherwise). For the same overall coupling strength, long-range interactions were found to produce a faster and more robust synchronization than the local coupling topologies (regular or random).

In a related approach, a fractal connectivity was proposed [512]. In such topology, two sites were connected with a probability dependent on the internode distance as $p_{i j}=r_{i j}^{\alpha}$, where the distance between nodes $i$ and $j$ is denoted as $r_{i j}$. This coupling scheme depends on the parameter $\alpha$ which defines the extent of the interaction neighborhood as well as the strength of coupling. Although this approach was applied to coupled chaotic maps, an enhancement of synchronization was also found by using long range interactions [512].

\section{Applications}

In this chapter we discuss a series of applications to real networks. These include both issues concerning the structure of the networks and their dynamics. We shall review some structural aspects of social networks and consider two kind of dynamics involving social networks: opinion formation and game models. We shall then discuss the statistical properties of the Internet and of the World Wide Web. Finally we shall focus of complex networks of interest to biology and medicine, such as networks describing the interactions between cell components, and neural networks.

\subsection{Social networks}

A social network is a set of actors (individuals or social groups) and relationships of different kinds (friendship, kinship, status, sexual, business or political) among them $[18,19]$. The quantitative analysis of social interactions goes back to the early 1920s [513]. The study of the school child's choice of companions reported by Wellman in 1926 [514] and the Mayo's study of social interactions between factory workers in the 1930s [19] are some examples of pioneering works in social network analysis. The cross-interaction of researchers form diverse disciplines as sociology, applied anthropology, social psychology and statistics has raised over the years a solid scientific discipline with its own textbooks [18,19], and specialized journals as Social Networks published by Elsevier.

As discussed in Chapter 2, many of the fundamental concepts (such as the small-world property) and tools currently used by the physicists in the analysis of complex networks have their origins in sociometry. It is the case, e.g., of the clustering index [515], or of the different measures of node centrality proposed in sociometry to quantify the social importance of a given individual in a network [18,516,517]. Centralities based on degree or on betweenness are some examples of such indices [38,39,517-520]. Some current problems in network analysis, as the characterization of a node by its relations, have also been raised in sociometric studies: several methods have been developed to quantify similarities between actors, based exclusively on the topology [521,522] (see Section 7.1.2). Concepts as the role or the equivalence of individuals were developed to locate actors placed similarly in a social network with respect to their set of relations [18]. Yet other problems such as searchability in networks, discussed in Section 7.2, has been started by sociological experiments [90,523], and measures such as the integration and the radiality have been proposed to quantify the degree an individual is connected and reachable within a given network [524].

\subsubsection{Structure}

Graph theory has been largely used to describe different social systems as friendship, affiliation and collaboration networks. 
The statistical analysis of real friendship networks has revealed the important interaction between friendships and adolescence delinquency [525]. The quantification of social interactions has even suggested an underlying network organization of conspiracy [526] or of terrorist cells [527,528]. Within the framework of complex networks there have been some attempts to characterize the social interactions in animals (association, aggression, submission, grooming) [529-531]; the networked memberships of football players, musicians, and movie actors [532-535]; or the interactions of fictional characters, such as the personages of Victor Hugo's Les Misérables, Tolstoy's Anna Karenina or Shakespeare's plays [51,536].

The fast development of communication systems, such as the telephone or the Internet, has generated new forms of social contacts [537,538]. Network theory has provided not only a statistical characterization of these virtual contacts, but also a better knowledge on the process forming such interactions. Some examples are the studies on phone and e-mail address books and reply networks [539-541], on phone calls [542], and on e-mail exchanges or other communications on the Internet $[84,543,544]$.

Scientific coauthorships are an interesting example of collaboration networks that has provided a better understanding of the social mechanisms ruling collaborations among scientists [545]. Coauthorship networks are constructed by connecting two scientists (the nodes) if they have coauthored at least a manuscript. Networks constructed from databases of different disciplines, as Medline or the Stanford Public Information Retrieval (SPIRES), have been found to display some non-trivial properties as small-world behavior or highly skewed degree distributions [40,52,184]. Related networks are the so called citation networks: scientific articles are associated to nodes and a direct link from A to B indicates that the article B is cited by A. Since the early works of Lotka in 1926 [546] and Shockley in 1957 [547], the statistics of citations has been an object of great interest. In a later approach, a non-trivial power law was observed in both the in- and out-degree distribution of a citation network [141]. Since then, the statistical properties of the distribution of citations have been discussed in different manuscripts [548-550], and recently the power law behavior of the in-degree distribution suggested the introduction of a new indicator (the index $h$ ) quantifying the impact of a scientist on the modern scientific community [551,552].

As a final example we mention a bioproduct of social interactions such as human language. Human languages have been studied by using graphs in which syllables or words are the nodes, whereas synonymous or syntaxes constitute the links. Even the elements of a narrative have been considered as a network [553]. Different studies have reported a non-random structure, characterized by small-world behavior, scaling in the degree distribution and disassortative mixing, in different semantic webs in languages as Portuguese, English, Czech German and Rumanian [554-560].

The paper by Rapoport [561] is one of the first works revealing the importance of the degree distribution in the propagation of an information. More recently, an abundant literature on affiliation networks has shown that the structure of group memberships plays a major role in the spread of attitudes or opinions, which may affect, for instance, political orientations or collective actions [183,562-564].

An example is the study reported in Ref. [562] in which the key political and economical role played by the well known Medici family is attributed to its central position in the network of the elite families during the Renaissance state in Florence (Italy) illustrated in Fig. 6.1.

Similarly, the networked structure created by the board memberships has been found to strongly rule the spread of ideas and attitudes in the corporate world, which may have an impact on influences, alliances and investment strategies [565-568].

The study of transmission of infectious agents (by sexual contact, needling sharing for the drug users, etc.) in a population is another example in which the structure of the network has provided important information for the modelling of realistic dynamics (see Section 4.1). Results from real data have confirmed the key role of the topology of social contacts in the spread dynamics of different pathogens as hepatitis B virus (HBV), human immunodeficiency viruses (HIV), bacterial pneumonia, syphilis, chlamydia and gonorrhea. Fig. 6.2 illustrates the links between the three primary ethnic groups in a drug users network. In some cases (namely for HBV and HIV), the analysis of network connections has led to develop prevention or control actions by detecting high-risk sites [569-574].

Various studies have reported power-law distributions in the number of sexual contacts of real networks $[356,357,575,576]$. These results suggest that preferential attachment models can be an appealing approach to explain the underlying structure of sexual contacts. Nevertheless, some studies have also pointed out the statistical pitfalls of such models in explaining some of the real observations [577-579]. 


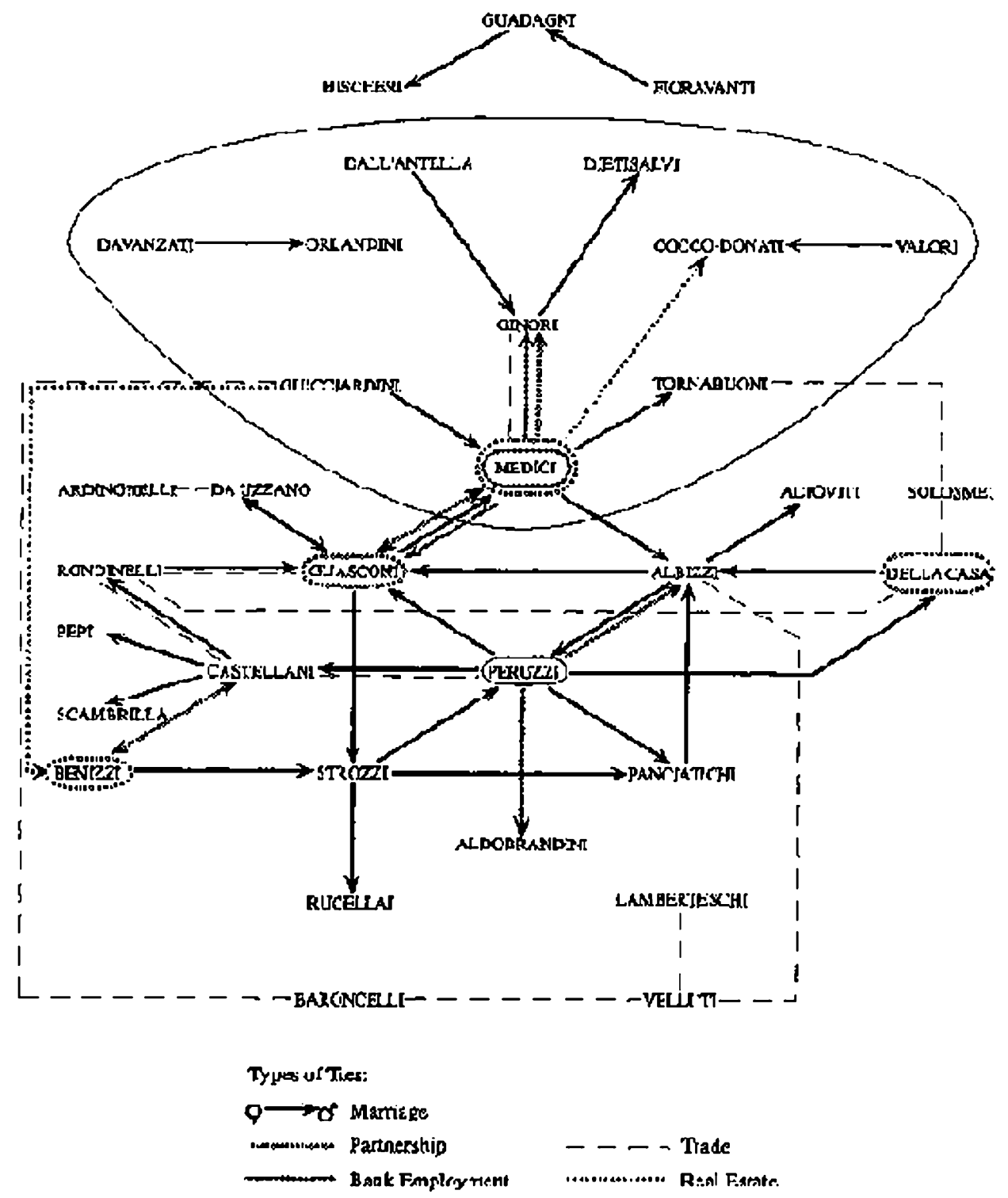

Fig. 6.1. Political and friendship structure between the elite florentine families during the Renaissance state in Florence.

\subsubsection{Dynamics I: opinion formation}

The application of Ising models and other tools of computational or statistical physics to social systems has a long tradition since the work by Majorana [580]. Here we shall discuss some of the models of opinion formation, such as those by Sznajd (S) [581], Deffuant et al. (D) [582], and Krause and Hegselmann (KH) [583], that have been recently studied in the context of complex networks. Other models, as the voter model [584-587], Galam's majority rule and the Axelrod multicultural model [588] will not be discussed.

The general idea behind opinion dynamics models is that individuals (or agents) have opinions that can change under the influence of other individuals. The interaction dynamics of the three models is governed by very simple deterministic rules [589-591]. In the $S$ model, the opinion $o_{i}$ of individual $i$ (with $i=1, \ldots, N$ ) is a binary variable assuming the value +1 or -1 . At each time step, two randomly selected neighboring agents transfer their opinion to their neighbors if and only if they share the same opinion [581]. In the $D$ model, opinions are real numbers $\left(0 \leqslant o_{i} \leqslant 1\right)$. At each time step, two randomly selected neighboring agents $i$ and $j$ check their opinions $o_{i}$ and $o_{j}$ to see if an exchange of opinion 


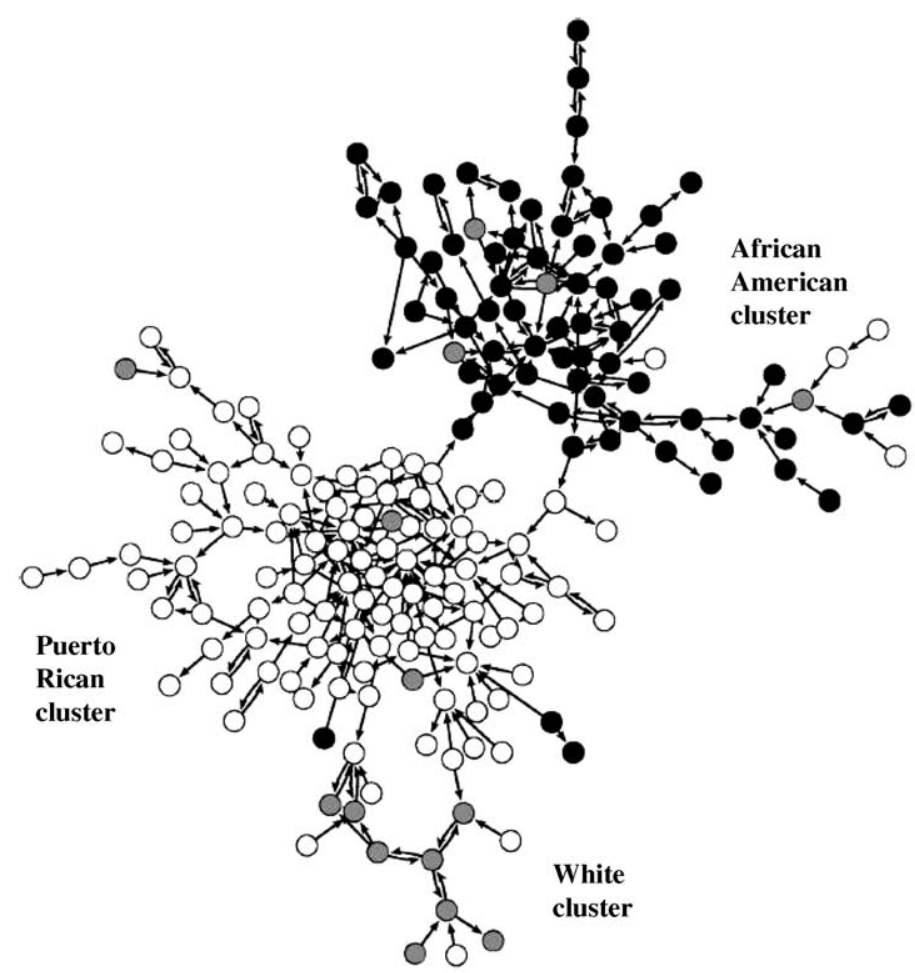

HRS study marco-network largest connected component by ethnicity. Total $n=193: \bullet$,African Americans $(\mathrm{n}=70)$; O, Puerto Ticans $(n=109)$; O, Whites/other $(n=14)$.

Fig. 6.2. From Ref. [570]. Structure and shape of the largest connected component of a drug users network. The nodes are clustered into the three primary ethnic groups considered in the analysis of Ref. [570].

is possible. If the two opinions differ by more than a fixed threshold parameter $\epsilon(0<\epsilon<1)$, called the confidence bound, both opinions remain unchanged. If, instead, $\left|o_{i}-o_{j}\right|<\epsilon$, then each opinion moves into the direction of the other by an amount $\mu \cdot\left|o_{i}-o_{j}\right|$, with $\mu$ being a second tunable parameter $(0<\mu \leqslant 1 / 2)$. The value $\mu=1 / 2$ corresponds to the case in which the two opinions take their average $\left(o_{i}-o_{j}\right) / 2$ [582]. In the KH model, opinions are also real numbers $\left(0 \leqslant o_{i} \leqslant 1\right)$. At each time step, an individual $i$ is chosen at random and changes its opinion into the arithmetic average of the opinions of all the neighboring agents that are within a confidence bound $\epsilon$ [583].

To see if and how a consensus emerges out of initially different opinions, the three models are started with a random initial distribution of opinions. The dynamics is followed until the system reaches an equilibrium state characterized by the existence of one or several opinion groups, according to the value of the control parameters of the models. For instance, the basic $\mathrm{S}$ model with random sequential updating always leads to a consensus on a regular lattice of any dimension $D$ (and even if more than two opinions are allowed). In particular, for any $D \neq 1$, one observes a phase transition as a function of the initial concentration $p$ of up spins. If $p=0.5$ then at the end of the dynamics half of the samples will have $o_{i}=+1 \forall i$ and the remaining half will have $o_{i}=-1 \forall i$. For $p<1 / 2$ all samples end up with $o_{i}=-1 \forall i$, while for $p>1 / 2$ they all end up in the other attractive fixed point $o_{i}=+1 \forall i$ [589]. In the $\mathrm{D}$ model, instead, all opinions converge to a single central one for $\epsilon>1 / 2$, while for $\epsilon<1 / 2$ different opinions survive, with a number of surviving opinions that varies as $1 / \epsilon$, as also supported by analytical arguments [592]. In the KH model, as $\epsilon$ departs from 0 , there is the formation of many groups, each one constituted by a few individuals sharing the same opinion. As $\epsilon$ becomes larger, the groups acquire more and more members, while their number is reduced. The coalescence of different groups takes place in a very similar fashion as the synchronization phenomenon of coupled oscillators described in Section 5.4 [593]. Eventually, for a critical value $\epsilon=\epsilon_{\mathfrak{c}}$, all the individuals reach a complete consensus. There are two possible values for the threshold depending on the average number of neighbors $\langle k\rangle$. If $\langle k\rangle$ grows with the number of agents in the population there is consensus for $\epsilon>\sim 0.2$. If $\langle k\rangle$ remains finite when the population diverges, the consensus threshold is $1 / 2$ as in the D model [591,594]. 
Only very recently the three consensus models have been implemented on top of complex networks, so that just a few and not conclusive results are currently available. However, the results already reported point to interesting dynamics of opinion formation.

Yu-Song et al. [595] have analyzed the transition from the state with no consensus to the state with complete consensus in the S model on small-world and scale free networks. Interestingly, they claim that the larger is the clustering coefficient, the easier the development of the consensus takes place. Notice also that if the $S$ dynamics is simultaneous to the growth of a BA scale-free network, complete consensus is no longer possible [596]. The greatest success of the $S$ model is the possibility to reproduce numerically some of the empirical laws observed in the results of political elections, such as a distribution of the number of candidates receiving $v$ votes each, that roughly varies as $1 / v$ (with systematic downward deviations for small and large $v$ ) [591]. For instance, the distribution of votes in the Brazilian elections was well reproduced by a version of the $\mathrm{S}$ model with many different opinions, instead of only 2 , on a BA scale-free network [597] and on a pseudo-fractal model [598].

Stauffer and Meyer-Ortmanns have studied the D model on a BA network [599]. They have found complete consensus for large $\epsilon$, and a number of opinions varying as $1 / \epsilon$ for small $\epsilon$, as for the D model on a complete graph. On the other hand, the number $O$ of different surviving opinions (if no complete consensus is achieved) is an extensive quantity, i.e., it varies proportionally to the number $N$ of agents, while it is intensive (independent of $N$ for large $N$ ) when everybody is connected to everybody [592]. The D model on a directed BA network exhibits the same properties as in the undirected case [599]. Moreover, the results do not change if the opinion dynamics is simultaneous to the growth of the BA network [600].

Concerning the KH model on complex topologies, Fortunato [601] has studied the model on scale-free networks in the context of damage spreading with the aim of understanding how opinion changes under targeted perturbations. He showed that in BA networks, there is a transition at a low value of the confidence bound parameter, whereas there also exists a critical $\epsilon$ value above which the initial perturbation reaches all other individuals in the system.

Finally, the role of well-connected leaders in the model on BA networks was studied in Ref. [602].

In conclusion, the role of complex topologies in opinion dynamics is still to be better investigated. The first steps already taken, and above mentioned, point to a new promising direction in the modelling of consensus formation.

\subsubsection{Dynamics II: strategic games}

A strategic game is a model of interacting decision-makers. More precisely a game consists of the following ingredients [603,604]: (i) at least two decision-makers (also known as players or agents) are involved in the game. These players: (ii) follow the rules of the game, (iii) have their own strategies, and (iv) play in a way to maximize their gain. Depending on whether or not the strategies are the same for each player, different game models can be defined. Furthermore, the games can have extra complications if the strategies are allowed to change in time. Game theory has been widely used to model society, economic systems, or biological evolution [605-607]. As for the models of opinion dynamics, a natural question that arose recently was what kind of new behaviors the interplay between complex topologies and game models would eventually produce.

One of the most well known strategic games is the Prisoners's Dilemma (PD) [608]. In one of its versions [609], the game is defined in the following way. Two players $A$ and $B$ meet repeatedly. At every iteration, each player has two options: to cooperate or to defect. If both players cooperate (defect), each of them receives one (zero) point. If a player cooperates and the other defects, the cooperator scores zero points while the defector scores $b$ points, with $b>1$. The spatial $P D$ consists of $N$ players on a network, usually taken as a regular lattice [609]. Each player plays an iterated PD game against each of its neighbors. The gain of a player is the sum of the gains from the encounters with its neighbors. If a neighboring opponent scores higher than the player, then in the next move the player follows the strategy of that opponent (this is the so called "tit-for-tat" strategy that is a successful strategy in the two-player PD [610]) with probability $1-p$, and, in order to model occasional irrational moves, the opposite strategy with probability $p$.

The spatial PD game has been considered on complex networks [611-616]. In particular, Kim et al. [612] have studied numerically the model on a 2D small-world network, considering the case in which there is an influential node with a finite density of directed random links to the other nodes in the network. They have shown that the degree of cooperation does not remain at a steady state level, rather it displays a punctuated equilibrium-type behavior manifested by the existence of sudden breakdowns of cooperation. The statistics for the frequency and duration of this instabilities has been also investigated. 


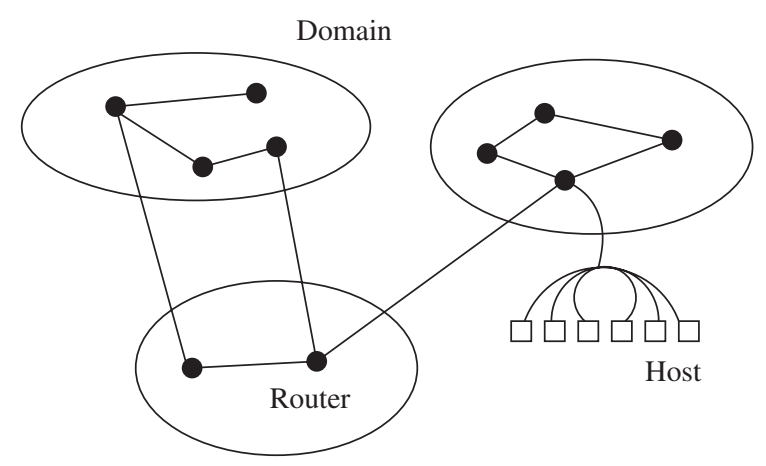

Fig. 6.3. Schematic diagram of the Internet. The large circles denote the domains, the small filled circles are the routers, and each unfilled square corresponds to a router host.

Later on, Holme et al. [614] have studied the spatial PD on the topology of real social systems. In this case, the time evolution of the cooperator density has an even more complex behavior than that of Ref. [612], despite the lack of directed links. The authors then argued that the crucial structural ingredients causing the observed behavior are the inhomogeneous degree distribution and the fact that the connections within vertices of highest degree are rather sparse. This indicates that the network structure can be as important as the game rules in order to maintain cooperative regimes. This finding has been recently supported by Lieberman et al. [616], who showed that the outcome of evolutionary games can entirely depend on the structure of the underlying graph. Given that game models have many fascinating applications, and that many real systems show complex topologies, it is expected that our understanding of cooperative behavior will deepen in the near future.

\subsection{The Internet and the World Wide Web}

In 1999, Faloutsos et al. [617] reported the first study on how the Internet map looks like. The Internet is a network in which the nodes represent the hosts and the links stand for physical connections among the hosts (see Fig. 6.3). Usually the Internet is described at two different scales: at the level of autonomous systems, or at the level of the routers. The Internet is formed, indeed, by many domains of different administrative control, such as network service providers or organizational units, commonly known as autonomous systems (AS). On its turn, each AS is divided into many subnetworks and the devices responsible for receiving and forwarding data packets, through both the subnetworks and among different AS, are called routers.

The main differences in the two descriptions from a technical point of view are the constraints associated to routing protocols at work. All routers are subjected to administrative routing policies and make decisions according to their routing protocols and the information they have. There are two different classes of special purpose routing protocols: the intra-domain routing protocol and the inter-domain routing protocol. The former is used to pass information within the same AS, while the latter serves for exchanging topological, path information and conditions on the network between peer routers in different AS. It is worth noticing at this point that, when mapping the Internet, one does not distinguish between private peering among border routers and the exchange of information through public exchange points, that is, one considers the ASs connected to each other, regardless on the details of the exchange points. As a consequence, one refers to routers without distinguishing between external routers and those forming internal subnetworks within As's. On the other hand, there are several topological differences between the AS representation of the Internet and the map emerging from routers connections, especially in the local properties such as degree-degree correlations (see Section 2.2).

Another communication network that has been recently characterized thoroughly is the World Wide Web (WWW), the network formed by hyperlinks between different Web pages. With more than $10^{8}$ known nodes, the WWW is the largest network ever mapped and, in contrast to the Internet, it is a directed network: each node represents a Web page which has a number of incoming links and also several outgoings links pointing to other Web pages.

Due to the spread of Internet connections among end-users, other kinds of technological networks have lately appeared. They are, in general, built up on top of existing networks like the Internet. This is the case of peer-to-peer 
Table 6.1

Basic properties of the Internet at the AS level for the year 1997, 1998, and 1999

\begin{tabular}{llll}
\hline Year & 1997 & 1998 & 1999 \\
\hline$N$ & 3112 & 3834 & 5287 \\
$K$ & 5450 & 6990 & 10,100 \\
$\langle k\rangle$ & 3.5 & 3.6 & 3.8 \\
$C$ & 0.18 & 0.21 & 0.24 \\
$\langle L\rangle$ & 3.8 & 3.8 & 3.7 \\
$\langle b\rangle / N$ & 2.4 & 2.3 & 2.2 \\
\hline
\end{tabular}

The reported quantities are: number of nodes $N$, number of edges $K$, average degree $\langle k\rangle$, clustering coefficient $C$, characteristic path length $\langle L\rangle$ and average betweenness $\langle b\rangle$. Data from Ref. [618].

networks, like Gnutella, that are becoming popular as a way to connect thousands of computers that allow sharing of files (for instance, music or video) between users over local and distant areas [99,379]. The efficient exploitation of these networks is in fact one of the most important technical issues, as they generally imply the use of large bandwidths. On the other hand, they are good candidates for modern marketing techniques such as viral marketing. Due to their intrinsic dynamical nature, such networks are more difficult to study than the Internet and the WWW.

The Internet and the WWW are interesting networks because of their large sizes that allow for a reliable statistical analysis of their topological properties. On the other hand, such networks are self-organized entities, combining diverse rules like human needs and environmental capabilities without a precise central design. For instance in the WWW, the contents of a single page and its hyperlinks are fully determined by the owner of the Web page, who collects similar information together, or connects to other personal Web pages according to social or collaboration relationships, etc. Notwithstanding the existence of diverse local rules, such networks exhibit some common properties, such as the small-world property and scale-free degree distributions. In addition, the Internet and the WWW are valuable objects because modern society increasingly depends on large communication networks. The need for information spreading pervades our lives and its efficient handling and delivery is becoming one of the most important practical problems. To this purpose, a suite of protocols for the dissemination of information from a given source to thousands of users has been developed in the last several years [8]. However, both the physical network and the numbers of users are growing continuously. The scalability of current protocols as well as their performance for larger system sizes and heavier loads are critical issues to be addressed in order to warrant the networks' functioning. Pushed by this practical need, developing realistic network models is crucial to test new protocols and predict traffic. From the other side, understanding the basic mechanisms of the formation of such networks is a crucial issue for designing infrastructures or protocols that optimize the cost and maximize the efficiency.

\subsubsection{Structure of the Internet}

Faloutsos et al. have analyzed the Internet AS maps collected from 1997 to 1998 by the National Laboratory for Applied Network Research (NLANR) [617]. Later on, other studies on the same data, together with those obtained at the router level [25,618], have further supported the results of Ref. [617]. The basic measures obtained are summarized in Table 6.1.

Fig. 6.4a shows the distribution of shortest path lengths $L$, while in Fig. 6.4b the normalized number of hops $M(L) / N$, where $M(L)$ is the number of nodes within a distance less than or equal to $L$ from any given vertex, is reported as a function of $L$. Notice that the two quantities are related since $M(L) / N=\sum_{L^{\prime}=0}^{L} P\left(L^{\prime}\right)$. The distribution probability $P(L)$ is characterized by a sharp peak around its average value $\langle L\rangle \approx 4$ and its shape remains essentially unchanged from the AS97 to the AS99 maps. Hence, shortest path lengths do not show strong fluctuations. This is confirmed by the hop plot in which $M(L)$ practically reaches its maximum value equal to $N$ at $\langle L\rangle \approx 5$. However, the plot does not allow to draw a definitive conclusion whether the behavior of $M(L)$ as a function of $L$ is a power-law or exponential [617,618] (see Section 2.5.1).

Concerning the degree, Table 6.1 indicates that the average degree is time independent, even if the number of nodes is more than doubled in the three-year period considered. The same result is confirmed by the plot of the degree distributions, shown in Fig. 2.4 of Section 2.2. As already discussed in Section 2.2, the degree-degree correlations are different depending on whether one is considering the Internet at the AS level or at the router level. 

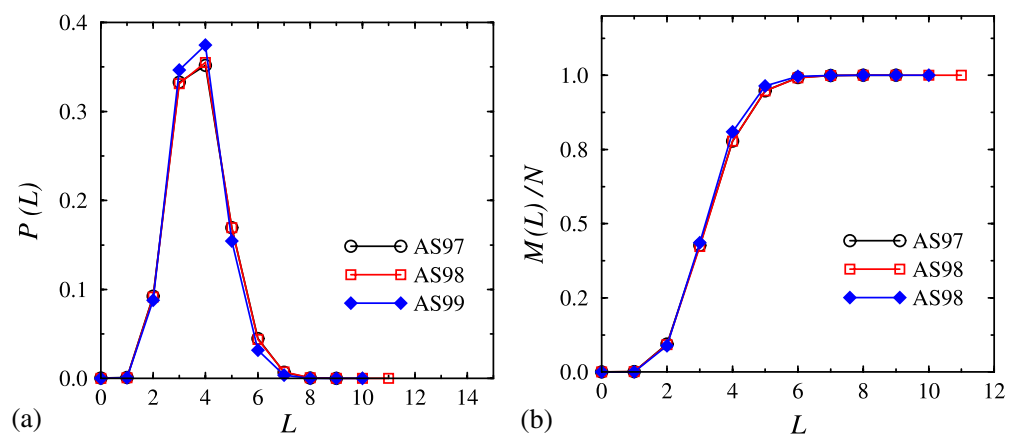

Fig. 6.4. Path length distribution $P(L)$ (a) and normalized hop plots (b) vs. $L$ for the Internet network at the AS level, for 1997 (AS97, circles), 1998 (AS98, squares), and 1999 (AS99, diamonds). Data collected by the National Laboratory for Applied Network Research (NLANR). Reprinted figure with permission from Ref. [618]. (C) 2002 by the American Physical Society.
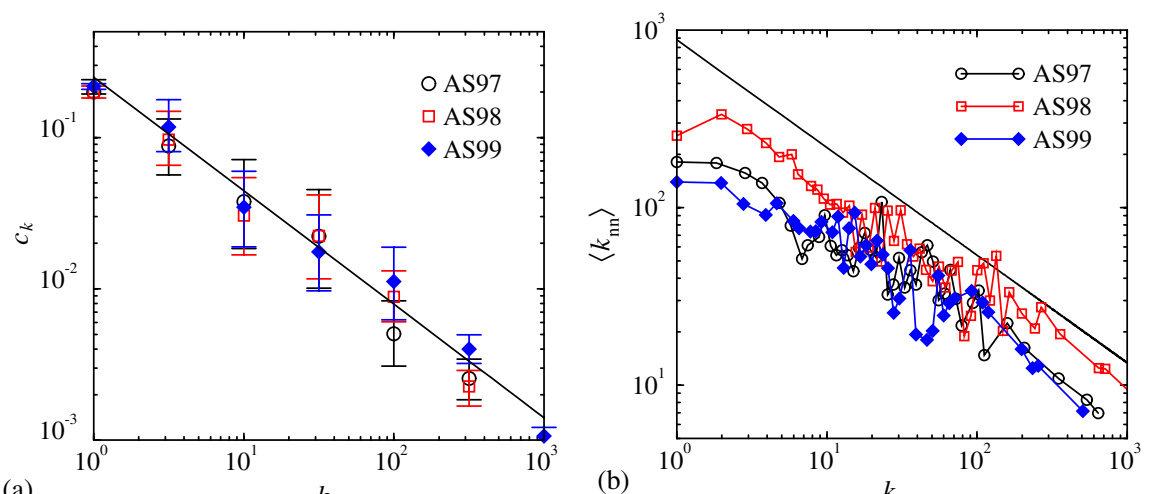

(a)

(b)
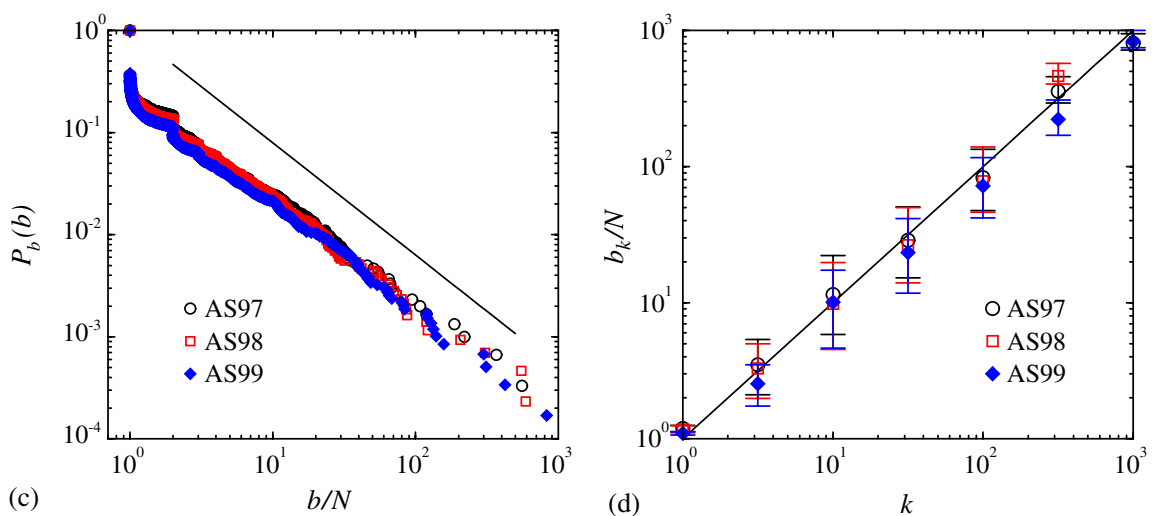

Fig. 6.5. Clustering coefficient $C_{k}$ as a function of degree $k$ (a), average degree of the nearest neighbors of a node with degree $k$ vs. $k$ (b), integrated betweenness probability distribution (c), and normalized betweenness as a function of the degree (d), for the 1997, 1998, and 1999 AS Internet networks. Reprinted figure with permission from Ref. [618]. (C) 2002 by the American Physical Society.

In particular, an explanation of the disassortative correlation observed at the AS level (see Fig. 6.5b) was proposed by Maslov et al. [619,620], who observed that the anti-correlation between the degrees of adjacent vertices has its origin in the restriction that two vertices cannot have more than one edge connecting them. Park and Newman analytically confirmed that this mechanism is indeed responsible for inducing the correlation properties of the Internet $[26,621]$. 
Table 6.2

Basic properties of the WWW

\begin{tabular}{|c|c|c|c|c|c|c|c|c|}
\hline Network & $N$ & $\langle k\rangle$ & $\gamma$ & $\gamma_{\text {in }}$ & $\gamma_{\text {out }}$ & $C$ & $L$ & Ref. \\
\hline WWW(Web page) & 325,729 & 4.51 & & 2.1 & 2.45 & & 11.2 & {$[623]$} \\
\hline WWW(Web page) & $4 \times 10^{7}$ & 7 & & 2.1 & 2.38 & & & [627] \\
\hline WWW(Web page) & $2 \times 10^{8}$ & 7.5 & & 2.1 & 2.72 & & 16 & [101] \\
\hline WWW(site) & 153,217 & 35.21 & & & & 0.11 & 3.1 & {$[628]$} \\
\hline WWW(site) & $26 \times 10^{4}$ & & 1.94 & & & & & [629] \\
\hline
\end{tabular}

The reported quantities are: number of nodes $N$, average degree $\langle k\rangle$, exponent of the degree distribution $\gamma$ (or $\gamma_{\text {in }}$ and $\gamma_{\text {out }}$ in all cases in which the network is directed), clustering coefficient $C$, and characteristic path length $L$.

As shown in Table 6.1, the Internet has a relatively high clustering coefficient, about 100 times larger than that of a ER random graph with the same size. Interestingly, the clustering properties increase with time. Moreover, as shown in Fig. 6.5a, the clustering coefficient is a power-law decaying function of the degree, $C_{k} \sim k^{-\omega}$, with an exponent $\omega=0.75 \pm 0.03$ [618]. This result has been interpreted as due to the modular structure, through which many small AS in a country might be interconnected forming clusters, while a few others will connect to sparse global areas.

Finally, the betweenness distributions follow a power-law $P(b) \sim b^{-\delta}$ with $\delta=2.1 \pm 0.2$, as indicated by the cumulative distributions reported in Fig. 6.5c. Moreover, as shown in Fig. 6.5d, the betweenness $b_{k}$ of nodes with connectivity $k$ seems to scale linearly with the degree, $b_{k} \sim k^{1}$ [618].

All the previous quantities can also be measured at the router level. While in most of the cases the results are in agreement with those found at the AS level, in general, the scalings are not so well-shaped. In particular, the clustering coefficient shows independence on the degree [165].

More detailed information on the structure of the Internet can be found in Ref. [8].

\subsubsection{Structure of the World Wide Web}

As mentioned before, the WWW can be studied at the web-page level, where a node corresponds to a web-page and the hyper-links are mapped into directed links between nodes. In this case, the degree of a node is made of incoming and outgoing connections. Another possible resolution is the site level, where a node corresponds to a site having a collection of Web pages, and two nodes are connected by undirected edges when there exist hyper-links between Web pages in the corresponding sites.

The computer science community already knew about the existence of power-law distributions in several measurable quantities, such as web requests [622] and the number of pages in a site [151]. Albert et al. were the first to find powerlaws in the degree distributions of Web pages in the domain *.nd.edu [623]. Because of the directed nature of the edges, the in- and the out-degree distributions are to be considered separately. The graph with about $3 \times 10^{5}$ Web pages in the domain *.nd.edu showed power-law decays $P_{\text {in }}(k) \sim k^{-\gamma_{\text {in }}}$ and $P_{\text {out }} \sim k^{-\gamma_{\text {out }}}$ with $\gamma_{\text {in }}=2.1$ and $\gamma_{\text {out }}=2.45$. Table 6.2 summarizes some of the basic properties of a series of networks describing the WWW, both at the Web page and at the site level. In general, the out-degree distributions appear to have a bending from a pure power-law behavior. This bending could be the signature of an exponential cut-off whose origin can be explained by the different nature of the in- and out-degree evolution. In principle there is no limit to the number of incoming hyper-links, that is determined only by the popularity of the Web page itself. On the contrary, the out-degree is determined by the number of hyper-links present in the page, which are controlled by the owner of the Web page. For evident reasons, it will be very unlikely to find an excessively large number of hyper-links in a given page. This represents a sort of finite capacity for the formation of outgoing hyper-links that might naturally lead to a finite cut-off in the out-degree distribution [83].

One noticeable fact concerning the exponents is that $\gamma_{\text {in }}$ is the same despite the different size of the three Web page networks considered, while $\gamma_{\text {out }}$ shows large deviations. Kahng et al. have argued that in multiplicative stochastic models, $\gamma_{\text {in }} \sim 2.0$ can be universally constant, while $\gamma_{\text {out }}$ shows fluctuating values [624]. Similar results were observed by an independent analysis [625].

Interestingly, the data about the power-law degree behavior can be refined by looking at thematic groups of Web pages. The WWW can be naturally decomposed in unified groups of pages, identified by the category of the page contents. Pennock et al. have analyzed the in-degree distributions for the following four categories of Web pages: 
Table 6.3

In-degree distribution exponent in Web pages of homogeneous category

\begin{tabular}{lllll}
\hline & Computer Science & Universities & Companies & Newspapers \\
\hline$\gamma_{\text {in }}$ & 2.66 & 2.63 & 2.05 & 2.05 \\
\hline
\end{tabular}

Such values should be compared with $\gamma_{\text {in }}=2.1$, obtained for the whole network. Data from Ref. [626].

computer science, universities, companies and newspapers [626]. The power-law fits give quite variable exponents, ranging from $\gamma_{\text {in }} \simeq 2.1$ to 2.6 , as shown in Table 6.3. Such a variation in the measured exponents could signal slightly different dynamics depending on the subject category.

\subsubsection{Dynamics}

Some important aspects on the dynamics of the Internet, as routing strategies and congestion effects, have already been treated in Section 3.2.2. In the WWW, differently from the Internet, each node contains information.

Retrieving a specific information from the WWW can be quite a difficult task without web search engines. The functions of a web search engine is to retrieve information on the majority of Web pages and to categorize them accordingly to the contained information. For the data mining in WWW, it is necessary to estimate the accuracy and valueness of such information. Interestingly, in the commercial search engine Google such estimation is performed by the so called "page rank" measurements, that are based on the topology of neighboring Web pages. Recently, the topological properties of the WWW have been studied from the viewpoint of data mining [630,631]. Menczer has investigated the connection between the linkage and the content of Web pages [632]. He has shown that the relationship between a text-induced distance metric and a link-based neighborhood probability distribution displays a phase transition between a regime where the linkage is not determined by the content and another where the linkage decays accordingly to a power law.

We will come back to some general ideas on searching processes in Section 7.2. Here we focus instead on a different aspect of the dynamics that concerns the fluctuation of fluxes over the networks. Argollo de Menezes and Barabási have measured the flux of information from daily traffic on a Internet router, and the number of visits to specific web sites, revealing that the characteristic coupling between average flux $\left\langle f_{i}\right\rangle$ and dispersion $\sigma_{i}$ of individual nodes obeys the universal law $\sigma \sim\langle f\rangle^{\alpha}$ [324,633]. The exponent $\alpha$ assumes two different values: $\alpha_{\text {in }}=\frac{1}{2}$ for the Internet, and $\alpha_{\mathrm{ex}}=1$ for the WWW. It is worth noticing that these results are independent of the specific network topologies. The existence of the two different universality classes can be explained by the competition between the system's internal collective dynamics and the changes in the external environment. In particular, the authors have modeled the traffic flow by a random diffusion of walkers and the diffusive dynamics by a directed flow process between randomly selected pairs of nodes. Numerical simulations have shown that, in the absence of an external stochastic influence, one gets $\alpha_{\text {in }}$, whereas, when external fluctuations become dominant, the dynamics produces $\alpha_{\mathrm{ex}}$. A direct consequence of such an observation is that, in the case of the Internet, the fluctuations solely come from the local dynamics, whereas the WWW is strongly influenced by the external environment.

\subsection{Metabolic, protein, and genetic networks}

In 1999, Hartwell and collaborators published an influential paper discussing the new challenges of modern biology [634]. The authors pointed out that an issue of utmost importance is to develop a general framework in which biological functions could be understood as part of a complex modular organization of molecules or cell's constituents. In other words, modern biology should explain not only the functioning of individual cellular components, but also how these components are interconnected through a complex web of interactions leading to the function of a living cell. It is then natural to ask what these biological networks at the cell organization level look like and how their structure couples to the dynamics.

Cells are life's fundamental units of structure and function. A cell is composed of thousand of molecules with inhomogeneous structure. All the activities of a living cell are executed following the master plan stored in its DNA. It was then expected that, once the complete instructions encoded in DNA would have been interpreted, one could map a gene (the basic information unit in the DNA) into a specific activity or function, with all the consequent potential 
applications such as targeted drug development [635]. On the contrary, although today the complete knowledge on the genes of several organisms is available, yet the relationship between blueprints in DNA and functional activities of the cell is not fully understood. For instance, the p53 gene and protein (having function to control cell's life and death) are known as tumor-suppressor, since it was found that the $\mathrm{p} 53$ protein does not function correctly in most human cancers. However, despite the many studies performed on p53 gene and protein, yet the way on how effectively suppressing the growth of cancer cells is missing at a genetic level. Recently, it has been proposed that the understanding of such cancer cell growth mechanism would be gathered not only from the study of the p53 gene and protein, but taking into account the whole network interacting with them [636]. That is, the function of the gene should be analyzed through a network in which the gene participates. Similarly to p53 network case, several other observations prove that some functional activities of the cell emerge from interactions between different cell's components through complex webs. Moreover, it is expected that the large-scale network approach may lead to new insights on various longstanding questions on life, such as robustness to external perturbations, adaptation to external circumstances, and even hidden underlying design principles of evolution.

Before discussing the properties of cellular networks, we present a brief introduction to cellular systems, mainly on the interactions between cellular elements without considering the spatial structure. More detailed accounts on structural and functional information can be found in Refs. [637,638].

The essential energy and substrates for the activity of a cell are obtained by breaking down complex molecules into small ones (for example, the series of biochemical reactions which convert glucose into simpler molecules). Conversely, most functions of a cell are performed directly or indirectly by building complex molecules from simple ones. The biochemical reactions involving transformation of matter and energy are classified as metabolism, and the participating substrates are called metabolites. Metabolic reactions might be controlled and balanced, and most of them have excessive energy barriers to occur in natural environments (without the action of some catalysts).

Metabolic reactions are catalyzed and regulated by enzymes. Most enzymes are proteins, which are biological polymers (chain of simple units, called monomers) constructed from 20 different kinds of amino acids. The function of a protein is determined by a three-dimensional folding structure attributed by the sequence of amino acids. Proteins have modular units called domains, which have compactly folded globular structures. Domains are often connected with open lengths of polypeptide chains. Furthermore, individual proteins often serve as subunits for the formation of larger molecules, called protein assemblies or protein complexes. Proteins are synthesized by ribosomes. The sequence of amino acids for a specific protein is determined exactly by the information encoded in the matching gene.

A gene is a discrete unit of hereditary information, consisting of a nucleotide sequence in the DNA. Hundreds or thousands of genes are arranged along the DNA's length. The ribosomes synthesize a specific protein, guided by the sequence of nucleic acid in the mRNA transcripted from a gene. The transcription of a gene to the mRNA is performed by RNA polymerases. In some cases, transcription by RNA polymerases can be initiated together with a transcription factor or by activator, and it can be suppressed by some repressors in various ways. Transcription factors, activators and repressors are proteins or protein complexes. Therefore, the synthesis of some proteins can be activated or inhibited by other proteins. As a result, transcriptions and protein synthesis are also regulated by the interaction among genes and proteins. Fig. 6.6 illustrates these schematic relationships.

On the other hand, a cell responds to external signals through reception, signal transduction, and response. For instance, a signaling molecule attaches to the matching protein (reception), the signal propagates along a pathway (transduction) by a series of interactions between proteins, and eventually specific transcription procedures are activated. The transcription factor activates a transcription of a specific gene (response), and the resulting mRNA synthesizes a protein by the ribosomes, which have the function of responding to the signaling molecule. Therefore, cell's reaction and functioning have close relations with the protein interactions and gene transcriptions. The simplest cases of such response are schematically illustrated in Fig. 6.7.

\subsubsection{Structure}

Publicly available database reporting integrated metabolic pathways, such as

KEGG (http://www.genome.ad.jp/kegg), WIT (wit.mcs.anl.gov/wit) [639], EcoCyc (biocyc.org/ecocyc) [640], have allowed for a study of the structure of metabolic networks.

Jeong et al. have considered the metabolic reactions of 43 different organisms, representing the three domains of life, and have constructed directed graphs whose nodes are the metabolites and edges represent biochemical 


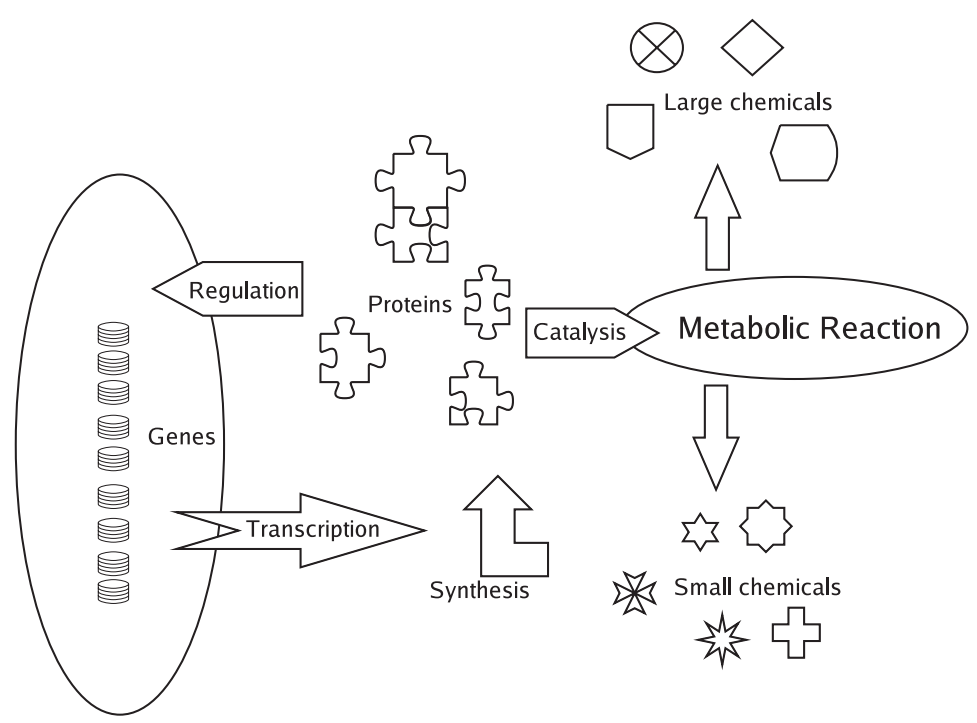

Fig. 6.6. Schematic diagram of interactions between the different cell's components.

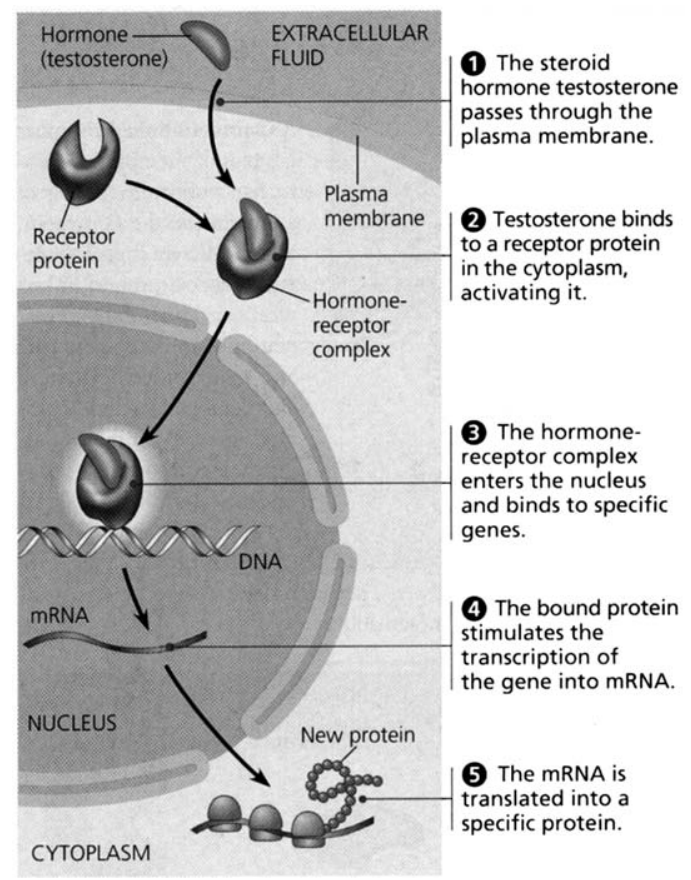

Fig. 6.7. An example of cell's responding activity to an external signal. Pictures taken from [637].

reactions [85]. A node receives an incoming edge when the corresponding metabolite is produced, and receives an outgoing edge when the metabolite is educed. Enzymes are not included in the graph. For all investigated organisms, the resulting graphs exhibit scale-free properties for both incoming and outgoing degree distributions. Fig. 6.8a shows the average degree distribution over the 43 considered species. The measured diameters of such metabolic networks are almost the same for different organisms, regardless of the number of metabolites found in the given species, as depicted in Fig. 6.8b, and exhibit small-world properties. 

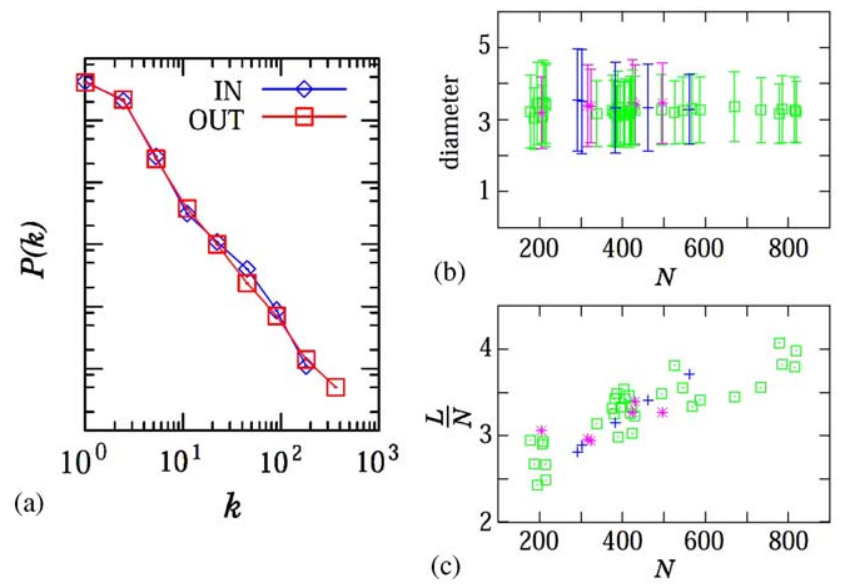

Fig. 6.8. (a) In- and out-degree distributions averaged over a set of 43 different organisms. (b) The diameter of the metabolic network as a function of the number $N$ of metabolites in the E. Coli. (c) The average number of outgoing edges vs. the number of nodes.

Scale-free degree distributions, small characteristic path lengths, and high clustering coefficients were found universally, irrespective of metabolic pathway databases and of the used methods to construct graphs from biochemical reactions. For example, instead of assuming virtual intermediate complexes, Wagner and Fell built up two networks (the metabolite and the reaction networks) from the metabolic pathways of $E$. coli [204]. The metabolite network consists of nodes representing metabolites and bidirectional links between educt and product of a metabolic reaction. On the other hand, the reaction network is the network where the nodes correspond to metabolic reactions and two nodes are linked when the two reactions share a metabolite. In metabolite networks, scale-free properties are detected, while the reaction network does not show power-law degree distributions. Small-world properties and relatively high clustering are found in both networks. Other studies with different ways of obtaining graphs show almost identical results [641-644].

Metabolism is known to have well defined modules having individual function such as catabolism, nucleic biosynthesis, lipidic biosynthesis, etc. The modular structure of the metabolic network was first studied by Ravasz et al. in Ref. [103]. A first evidence is that the clustering coefficients show independence on the network size and are larger than those pertinent to the BA-model. Moreover, the clustering coefficient averaged over nodes of the same degree decays as $C(k) \propto k^{-1}$. These properties of the clustering coefficient are similar to those of hierarchical network models, and indicate that the metabolic network cannot be partitioned into several definite modules, but there exists hierarchical structures such that several submodules combine into larger modules. To validate this argument for the metabolic network of $E$. Coli, hierarchical tree structures were extracted considering a metabolite or a group of metabolites as a submodule. The tree structure was obtained based on topological overlaps, because in a module the elements will form highly clustered structures and will share a large portion of neighbors, whereas elements belonging to different groups do not. It has been shown that the hierarchical structure resulting from the graph topology matched well to the biologically identified modules [645,646].

Another viewpoint on modular structures is the so called "bow-tie" structure of metabolic networks [642]. There, it is shown that the metabolites in a metabolic network are far from being fully connected. A connectivity structure consisting of four major subsets of metabolites and reactions (namely, a fully connected sub-network, a substrate subset, a product subset and an isolated subset) were found in metabolic networks of 65 fully sequenced organisms [642]. The largest fully connected part of a metabolic network, called "the giant strong component", represents the core of the network and has the feature of scale-free networks. The average path length of the whole network is primarily determined by that of the giant strong component. One of intriguing recent results on modular structure is that scalefree properties come from the carrier metabolites, which are densely connected to various functional modules, whereas other metabolites, most of which are tightly connected to inside modules, show almost exponential distributions in their degrees [644].

Another class of well-studied cellular networks is that of protein-protein and protein-gene interaction networks. Interactions among proteins have a crucial role in several functional activities, such as signal transduction. According 

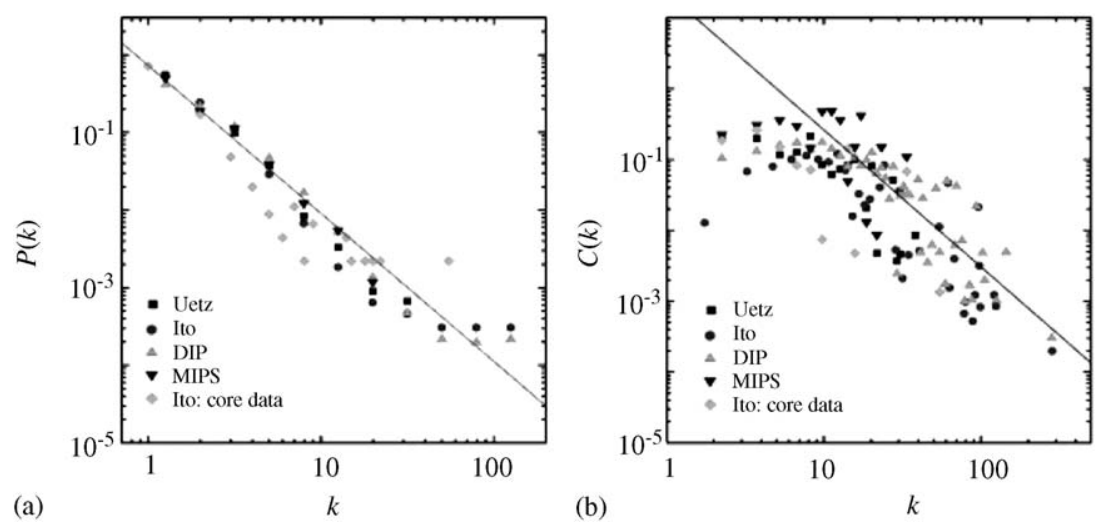

Fig. 6.9. (a) Degree distribution of networks from different data sets (specified in the legend). (b) Clustering coefficient as a function of the degree for the same data sets. In both plots, the straight lines have slope -2. Reprinted figures with permission from Ref. [647]. C) 2004 by Wiley.

to the demand of understanding the protein interaction map, several high-throughput experiments have been performed. They provide evidence of a partial interaction map between proteins (for review see [648-651] and references therein). In the graph representation, a node corresponds to a protein and two proteins are linked when they physically interact. The east two-hybrid screen method has been applied for revealing protein-protein interactions by Uetz et al. (http://portal.curagen.com) [652] and by Ito et al. [653]. Hand cured databases are available, such as MIPS (http://mips.gsf.de) [654] and DIP (http://dip.doe-mbi.ucla.edu) [655]. Similarly to metabolic networks, scale-free properties (shown in Fig. 6.9a), high-clustering and small-world properties with hierarchical modularity (shown in Fig. 6.9b) and robustness against random attacks were observed in protein-protein interaction networks $[86,647,656,657]$. It should be noticed that the databases used in the analysis show very small overlap, while the individual networks obtained from each database show a very similar structure. In particular, Yook et al. argued that the biological functional organization and the spatial cellular organization are correlated significantly with the topology of the network, by comparing the connectivity structure with that of randomized networks.

Protein crystallography reveals that the fundamental unit of the protein structure is the domain. From the functional point of view, the protein domain level structure can also be analyzed. Publicly available databases are the Prosite database (http://expasy.proteome.org.au/prosite/), the Pfam database (http://www.sanger.ac.uk/Software/ Pfam/index.shtml), the ProDom database (http://www.toulouse.inra.fr/prodom.html), the InterPro database (http://www.ebi.ac.uk/interpro), and the SCOP database (http://scop.mrc-lmb.cam.ac.uk/scop). The protein domain networks are formed by considering each domain as a node, and linking two nodes with an undirected edge, if the two domains appear together in a same protein. Wuchty et al. have highlighted the scale-free, small-world, and high clustering properties of domain networks [658]. Other studies have given evidence that the network of domains and evolutionary families have scale-free properties $[659,660]$.

Another experimental method to estimate activity of genes relies on micro-arrays, that provide measurements on how much a gene's expression appears at a given sampling for all possible genes. Therefore, the resulting data contain the expression level for various sampling conditions, and for various genes. Networks constructed from gene expression data are currently under exploration [107,260]. In particular, Agrawal studied networks from gene expression of cancer data. Individual gene expression level at different samples are regarded as a coordinate, and expressions of a gene map to a vector in $D$ dimension, when $D$ samples exist. The distances between genes are measured by the Euclidean norm between the two corresponding vectors. The networks are constructed by connecting vertices that belongs to each others' list of $K$ nearest neighbors in distance. On minimizing the order parameter with respect to $K$, the degree distribution of the network shows a power-law behavior in the tails with an exponent 1 . The eigenvalue spectrum of the adjacency matrix shows similarity to that of scale-free networks. Stuart et al. have further shown that co-expressed gene networks of humans, flies, worms, and yeast have scale-free properties [107].

Maslov and Sneppen [205] have studied the features of protein interaction and gene-regulation networks of the yeast. From measured correlations in the connectivity, it was observed that the network is disassortative, that is, links between highly connected proteins are systematically suppressed, whereas those between highly connected and low-connected 
pairs of proteins are favored. The correlations in connectivity was measured by comparison with "null-model" networks, which are constructed by randomly reshuffling links, while keeping the same connectivity of all nodes.

Up to now, we have presented evidences supporting that scale-free distributions, small-world, and high clustering seem to be universal properties of cellular networks. Then, what do these properties mean in biological system? And what basic principles in biology give rise to such universal features?

One of the basic properties of scale-free networks is the existence of hub nodes. As shown in Chapter 3, hub nodes have a topologically crucial role against random errors, and (as one can see in technological networks such as the Internet and WWW) hubs are also important from a functional aspect. For the metabolite network, the key metabolites can be estimated by the graph topology, and the resulting key metabolites are well known to be the essential metabolites in biochemical literature [85,204]. Indeed, some of the metabolic hubs point to the remnants of the RNA world, such as the coenzyme A, NAD and GTP, as well as they are elements of some of the most ancient metabolic pathways, such as glycolysis and the tricarboxylic acid cycle. Protein interaction networks possess a high degree of tolerance against the removal of randomly selected nodes, while attacks on highly connected proteins can be lethal in the network's topology [86]. As for biological aspects, it is argued that the essentiality of reactions in a node is not correlated to the node connectivity [661].

These observations on hub nodes naturally lead to the concept that the evolution procedure may be related to the topological structure of cellular networks. The scale-freeness property of a network might indicate growing and preferential attachment as evolutionary principles that led to the formation of the network as we know it nowadays. Biological evolution is the combined procedure of random genetic duplications and natural selections. The growing procedure can be understood as the appearance of duplicated genes, so that the new protein obtained by such a duplication interacts preferentially with the existing proteins, when the new protein has almost identical connections with respect to the old one. Following these concepts, several theoretical and numerical studies show that the resulting networks exhibit properties similar to those of cellular networks [175-177,662-667]. These models have been validated in real data by Eisenberg and Levanon [668]. Furthermore, by a close investigation on the protein interaction map of $S$. Cerevisiae, it was shown that the older a protein is, the better it is connected, and the number of interactions a protein gains during its evolution is proportional to its connectivity.

Short path lengths between cellular components are considered to be related to fast responses and adaptation under changes of circumstances [204]. For instance, metabolic networks need to react to perturbations (such as changes in enzyme or metabolite concentrations). Since metabolic networks are highly connected, each component in the network may be affected by such perturbations, and thus the network as a whole must adapt to the changed conditions by assuming a different metabolic state. Any response to a perturbation and transition to a new metabolic state requires that information about the perturbation has to be spread within the network. Therefore, the small-world property provides a fast and efficient way of spreading the perturbation along the network, this way allowing a metabolism to react rapidly to a given change in the external conditions.

The modular structure of cellular networks is currently widely studied $[634,669,670]$. A cell consists of spatially or chemically isolated functional modules, highly integrated by cellular components, and carrying discrete functions. A functional module is a discrete entity, whose function is separable from those of other modules. This separation depends on chemical isolation, which can originate from spatial localization or from chemical specificity. Modules can be insulated from (or connected to) each other. Insulation allows the cell to carry out many diverse reactions without cross talk that would harm the cell, whereas connectivity allows one function to influence another one. Moreover, modular structures have also consequences on the easiness for reconfiguring or adapting to new conditions, and on the robustness to component tolerances. Several studies have attempted to identifying such modules from the topology of the network [646,671-676], or to combining the topology with the integrated functional genomics data [657,677-681]. One relevant result on modular structure of cellular networks is the assortativeness shown in [205,644]. Suppressing a link between highly connected proteins and favoring links between highly connected and low-connected pairs of proteins decreases the likelihood of cross talks between different functional modules of the cell, and increases the overall robustness of the network against localized effects of deleterious perturbations. This feature (combined with scale-free and small-world property) is considered nowadays a key property to explain biological robustness [105].

Finally, we mention that there exist also several studies on motif structures in cellular networks $[108,682,683]$. In particular, transcription networks were found to be built of a small set of network motifs. Thus they are much simpler in structure (have fewer types of subgraphs) than might be thought. Each network motif appears to carry out a specific dynamical function in the network, as it has been experimentally and theoretically demonstrated [887]. For example, 
feed-forward loops act as sign-sensitive filter elements, pulse generators and response accelerators. Single input modules act as temporal pattern generators [887], etc. This raises the hope that network motifs can serve as elementary circuit elements for understanding the network dynamics [888]. Csete and Doyle argued that the scale-free and the bow-tie structure come from an effective trade-off among efficiency, robustness and evolvability, with predictable fragilities that can be used to understand disease pathogenesis [684].

\subsubsection{Dynamics}

During the last several years a wealth of experimental data, obtained with technological advances such as cDNA microarrays, have allowed the dynamical characterization of several biological processes both on a genome-wide and on a multi-gene scales and with fine time resolution. From a theoretical side, compelling models on the dynamics governing metabolic and genetic processes are hard to build as these biological phenomena are highly nonlinear and with many degrees of freedom. However, scientist have certainly advanced towards a comprehensive global understanding of, for instance, gene regulation through genetic engineering that require a thorough understanding of the general principles that can guide the design process. It is impossible here to provide an exhaustive review of the subject. However, we think that it is important to provide at least some ideas about the research lines that relate the structure and the function of biological systems.

Concepts such as operon, regulator gene and transcriptional repression were first introduced in the literature by Jacob and Monod [685]. Their model has served as the basis for more elaborated models as different regulatory mechanisms have been discovered [670]. Recent theoretical studies capitalize on these kind of models in order to elucidate what are the system constituents, their properties and how they interact in order to give rise to the collective behavior of the system. The final goal is to understand the relationship between structure and function as determined by the biological environment. In this sense, different gene circuit designs should be compared to determine which of them confers selective advantage in an ecological context and thus one should be able to advance what the functional consequences of different designs are. This is usually done by exploring the parameter space and looking for performance criteria such as the ability of a system to return to a steady state after a perturbation (called stability) or its responsiveness, that can be measured as the recovery time of the system after an environmental change (a change in an independent variable).

The results obtained for elementary gene circuits certainly provide answers to intriguing questions about how gene circuits could be organized, but at the same time pose new ones. With the recent advances in the characterization of the structure of gene networks, it is clear that genome-wide approaches will allow to discover new higher-order patterns. Therefore, more efforts in modeling the dynamics of increasingly complex gene circuits are expected in the near future. Some steps in this direction have been given.

Recently, Gómez-Gardeñes et al. [686] have analyzed a model with activatory and inhibitory interactions that generically describes biological processes (as metabolic reactions and gene expression) and that can be used to explore the conditions for the existence of stable fixed points and periodic states in heterogeneous networks. Specifically, the activity of the nodes is described by the vector $\mathbf{G}(t)=\left\{g_{1}(t), g_{2}(t), \ldots, g_{N}(t)\right\}$, where $g_{i}(i=1, \ldots, N)$ accounts for the activity level of each individual node $i$ in a network made up of $N$ elements. The time evolution of $\mathbf{G}(t)$ is described by the following set of first-order differential equations:

$$
\frac{\mathrm{d} \mathbf{G}(t)}{\mathrm{d} t}=-\mathbf{G}(t)+\mathbf{F}(\mathbf{G}(t))
$$

where $\mathbf{F}(\mathbf{G}(t))$ is some nonlinear term where the interactions between the network's elements are taken into account. In particular, a continuous Michaelis-Menten description has been implemented:

$$
F_{i}(\mathbf{G}(t))=\delta \frac{\Phi\left[h \sum_{j=1}^{k_{i}} W_{i j} g_{j}(t)\right]}{1+\Phi\left[h \sum_{j=1}^{k_{i}} W_{i j} g_{j}(t)\right]},
$$

where $W_{i j}$ is the interaction matrix that contains both the topological structure and the type of interactions. Additionally, $\delta>0$ and $h>0$ are constants, $k_{i}$ is the connectivity of node $i$, and $\Phi(z)$ is a function defined as $\Phi(z)=z$ if $z \geqslant 0$ and zero otherwise.

The above dynamics can be regarded as a generalization of some simplified and coarse-grained genetic models, referred to as random Boolean networks [687]. Random Boolean networks are based on Boolean rules and have been 
extensively used to study networks at various levels of biological organization, providing useful insights later supported by experiments [687,688]. Eq. (6.1) incorporates the experimental observation of a continuous range of activity levels. While linear models have been successful for the reconstruction of the interaction networks from experimental data, nonlinear models like Eq. (6.1) are expected to be more appropriate for a quantitative description of the dynamics. The dynamics (6.1) of a two-agent (dimer) model has been considered in Ref. [689], in the context of virus-cell interactions in bacteria and general gene regulatory activity models, where a rich repertoire of behaviors, like multistability (multiple attractors in phase space) was reported. As shown in [686], when the above dynamics is implemented in a large directed scale-free network, new features arise. The results indicate that complex dynamical patterns can exist in the form of periodic and chaotic orbits revealing interesting properties at both local and global levels. Moreover, the dynamics on top of the substrate networks yields topologically complex substructures (islands or clusters) whose structural characteristics are different to those of the original underlying network. These sub-networks of fluctuating activity are further divided in regions according to whether or not a local perturbation propagates throughout the system, indicating additional relationships between the structure and dynamics of networks of activatory-inhibitory elements.

In this context, each vertex of the network would represent a regulatory gene and the links would describe their interactions. In other words, two nodes at the ends of a link are considered to be transcriptional units which include a regulatory gene. One of these end-nodes can be thought of as being the source of an interaction (the output of a transcriptional unit). The second node represents the target binding site and at the same time the input of a second transcriptional unit. By studying simplified models as the one introduced above (the intrinsic complexity of the problem does not allow for a complete and detailed description of real gene dynamics), one can infer the region of the parameter space that better describes gene networks. Based on this hypothesis we would either guess dynamical interaction rules or provide hints for the experimental validation of the topology of real networks.

As for metabolic networks, the system of differential equations, Eqs. (6.1)-(6.2), represents one of the most basic biochemical reactions, where substrates and enzymes are involved in a reaction that produces a given product. In this context, there are several important issues as how fast the equilibrium is reached, how the concentration of substrates and enzymes compare, etc. Besides, it is known that in a large number of situations, some of the enzymes involved show periodic increments in their activity during division, and these reflect periodic changes in the rate of enzyme synthesis. This is achieved by regulatory mechanisms that necessarily require some kind of feedback control as that emerging in studies like [686]. Obviously, there is a vast literature on this kind of process, but the point here is that the real topological features of the underlying metabolic network should be incorporated in the current dynamical models.

Another approach that deserves to be mentioned is the study of dynamical networks of small genetic feed-back circuits. From recent research on biological systems (specifically on genetics) it appears evident that such dynamical feed-back networks are essential for understanding genetics expressions (for instance how densities of certain proteins vary in time), and they are necessary for modelling cell regulations and cell death under DNA damages. In particular, Refs. [690-692] have studied a series of dynamical aspects occurring in such networks by means of sets of differential equations.

In summary, while the complex network structures among cellular components are intensively discussed, the understanding of the temporal activity of a cell is still at an early stage. Some studies on the temporal activities of gene expression exist [693-695], and we believe that, based on the gathered knowledge on the topological features of these networks, the dynamical activities of cellular components will be an essential bench mark for future studies.

\subsection{Brain networks}

Neural assemblies (i.e. local networks of neurons transiently linked by selective interactions) are considered to be largely distributed and linked to form a Web-like structure of the brain [696]. The resulting network, extremely sparse, is capable to coordinate and integrate distributed brain activities in a unified neural process. The interplay between neuron organizations and neural functions was pointed out in the early work of Ramon y' Cajal (for a review see [697,698]). The most important point that is addressed is whether the structure of the neural wiring is related to brain functions.

Despite the huge number of neurons and their interconnections in human brain cortex $\left(\sim 10^{11}\right.$ neurons and $\sim 10^{14}$ synapses), the brain organization is ruled by optimizing principles of resource allocation and constraint minimization [699]. In the course of the evolution of the brain, the number of neurons has considerably increased, whereas their connections have become less direct [700]. Connectivity of cortical areas would lead to minimize costs of interconnection 
between neural sites, thus yielding an efficient communication [701-705]. Evidence exists that human cerebral cortex is an ensemble of clusters of densely and reciprocally coupled cortical areas that are globally interconnected to form a large-scale cortical circuit [696,706]. However, as the intrinsic properties of each neuron vary over time (driven by the ongoing interactions with the environment and with other neural assemblies), neuron dynamics changes continuously. This plasticity renders neurons able to continuously change connections, or establish new ones according to the computational and communication needs. Interactions between neural elements become therefore highly dynamic [707]. This dynamic interaction between distant cortical regions would underly the emergence of coherent global dynamics. Evidence has lead to the hypothesis that a functional interaction (time-dependent), and not only a simultaneous activation of neural populations, is a basic mechanism for the so called large-scale integration problem $[696,706,708,709]$. By means of this mechanism, differentiated local populations of synchronized neurons (assemblies) are functionally integrated at multiples spatial and temporal scales as a unified neural process $[696,708,710]$.

\subsubsection{Structure}

Most of the recent works aim to quantify the role of connectivity in the computational and communication abilities of neural networks. However, the very same notion of connectivity is controversial since neural networks are observed at different spatial scales, ranging from individual neurons linked by synaptic connections, to relatively large brain areas interconnected by pathways. Furthermore, data used in brain connectivity studies differ from one another in the spatial and temporal resolution, and it is controversial whether a given data set reflects individual neuron activities, neural assemblies dynamics, or other macroscopic (electrical, magnetic or hemodynamic/metabolic) brain activities [711-713]. The literature usually refers to three different types of connectivity: neuroanatomical, functional and effective [714-716].

The neuroanatomical connectivity is meant as the description of the physical connections between two brain sites [714]. This connectivity is difficult to be formally defined because the connections of neurons can be modulated by the brain plasticity [707]. Furthermore, variability in the anatomical connections has been observed to depend on the experimental techniques [717]. For a complete description of neural connections, full information on, e.g. the receptor subtype at synapses, or the ratio of inhibitory/excitatory interneurons would be necessary. We are thus far from a complete anatomical description of neural networks.

The functional connectivity is often defined as the temporal correlation between spatially distant neurophysiological events [718]. At a microscopic level, the functional interactions of neurons are related to a temporal coherence (measured by cross-correlation tests) of their spike trains activities [719]. In contrast, functional interactions at a macroscopic level have been evaluated using brain data from electroencephalographic (EEG) and magnetoencephalographic (MEG) diagrams, local field potentials (LFP), functional magnetic resonance imaging (fMRI) or positron emission tomography (PET) recordings [696,706,718,720,721]. Major problems for a unified definition of functional connectivity are the different spatial and temporal resolution scales of the recorded dynamics and the fact that, due to the volume conduction in brain tissue, activities recorded at one site reflect the dynamics of the whole surrounding neighborhood of neurons.

The effective connectivity, a more abstract notion, is generally defined as the influence that a neural system may exert over another, either in a direct or an indirect way [718]. However, this type of connectivity does not reflect unequivocally the anatomical connections, because a different arrangement of neural sites and their connections may lead to the same overall behavior. A major problem of both functional and effective connectivity is the fact that they depend on the model used to quantify the interactions, or on the experimental setup (trial to trial variability, subject-subject variability, etc.) [714-716].

Most complete information of neuroanatomical connectivity is now available for large-scale networks at the level of segregated brain regions for some mammalian species [722,723]. Detailed information has been collected on the connectivity of the hippocampal system of the rat [724], of the cortico-cortical system in the macaque monkey [725,726] and in the cat [727,728], and of the cortico-thalamic system in the cat [729].

The case of the Caenorhabditis elegans constitutes an excellent experimental system for understanding the relationships between structure and function in an entire nervous system. The full potential of such system comes from the integration of both anatomical and electrophysiological data. The C. elegans has a compact nervous system of 302 identified neurons, whose connectivity has been fully mapped [730]. Furthermore, electrophysiological recordings have provided new clues to the understanding of the electrical properties of its sensory neurons [731].

In a pioneering work [725], a small matrix connection of primate visual cortex (32 brain areas and 305 connections) was analyzed by means of a hierarchical manual sorting. The aim was to classify connections as ascending, descending, 


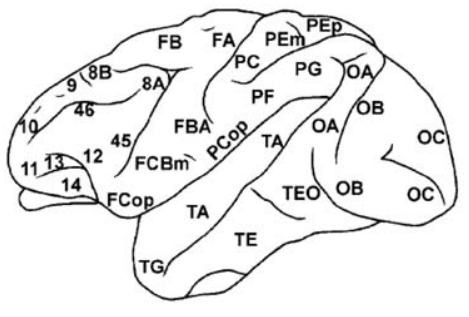

(A)

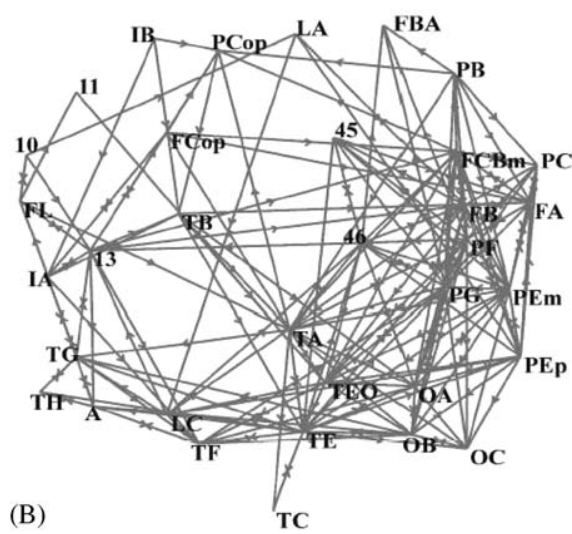

(B)

Fig. 6.10. Reprinted from Ref. [735]: (C) 2000 The Royal Society of London. (A) lateral representation of macaque cortex where the labels denote the approximative position of areas. (B) Example of a metric representation of the connections in the cortical network as obtained by NMDS.

or lateral, according to their patterns of origin and termination in the cortical layers. This hierarchical analysis required detailed a priori anatomical information about the direction of the patterns of projection. Such directions were used to place brain areas above, below or at the same level in a hierarchical graph. The result is the popular scheme represented in Ref. [736]. This hierarchical graph model was then re-studied by a computational method based on evolutionary algorithms [732]. The objective was to find an optimal hierarchy subjected to anatomical constraints. By an algorithm similar to the simulated annealing [267], several realizations of hierarchy models were evaluated, modified and selected following a cost function related to the anatomical constraints. The result of these approaches was a comprehensive representation of how the visual cortical areas are organized. However, an exact model of hierarchy has not yet been determined [732].

A comprehensive representation of several brain connectivity data sets was obtained by optimization methods [727,729,733-735]. By means of a non-parametric multidimensional scaling (NMDS), a graph was derived from the connectivity matrix, such that the distance between connected structures is minimized, whereas the distance between structures with no reported connections is maximized [521]. The distance between brain areas is generally an inverse measure for the degree of similarity of their connectivity patterns. The result is therefore a graph where nodes with similar patterns of connection are located together (as a cluster) whereas nodes with different connectivity patterns are located far apart. An example of the graph obtained by the NMDS method is depicted in Fig. 6.10. The organization into clusters has been evaluated by means of a non-parametric clustering [724,737] or a hierarchical clustering approach [735]. Although a careful statistical analysis is required in interpreting results from the NMDS of small data sets [738], the topology obtained suggested a clustering organization of cortical brain areas which is consistent with neurophysiological studies [724,727,729,733-735].

A comparison of hierarchical model and optimization method yielded very related organizations of areas in the cortical hierarchies [728]. By statistical comparisons, a simple connection rule (the strength of connections between brain areas is directly related to the number of common neighbors) was found to be a plausible mechanisms involved in the development of cortical connectivity [728,733]. Recently it has been suggested that the use of weighted connections in the construction of models of visual cortex may provide new insights into the functional organization of cortical circuits [739].

Network partitioning methods and different connectivity indices (computed directly from the adjacency matrix) were used to find functional clusters of brain areas. Briefly, the partitioning method operates in a graph by finding the optimal configuration which minimizes an intra-class inertia [740]. The placement of nodes in the space is such as the distance between two of them reflects the difference of their local connectivities. Results suggested a functional role played by some brain areas during the neural organization in vision [741].

The analysis of neural connectivity matrices has revealed that both the nervous system of the C. elegans [28], and various cortical networks [735,742-744] as well as in vitro neuronal networks [745], exhibit the small-world property. The values of the standard measures (characteristic path length and clustering coefficient) for some of the databases are reported in Table 6.4. Despite the existence of significative variations from one study to another, the 
Table 6.4

Number of nodes $N$, characteristic path length $L$, clustering coefficient $C$, and global efficiency $E_{\text {glob }}$, for some neural networks

\begin{tabular}{lrrll}
\hline Data set & $N$ & $L$ & $C$ & $E_{\text {glob }}$ \\
\hline C. elegans & 282 & 2.65 & 0.28 & 0.46 \\
In vitro neuronal network & 240 & 17.58 & 0.113 & Unknown \\
Macaque visual cortex & 32 & 1.69 & 0.59 & Unknown \\
Macaque cortex & 73 & 2.18 & 0.49 & 0.52 \\
Cat cortex & 55 & 1.79 & 0.60 & 0.69 \\
\hline
\end{tabular}

Values taken from Refs. [28,30,737,742,745].

small-world behavior was statistically assessed by a comparison to the values obtained for random graphs of the same sizes. Moreover, in different cortical connectivity matrices, significant correlations were observed between the values of the path length or the clustering coefficient and the number of neighbors of each individual brain region [743]. Other topological indices as the global efficiency [30], the fraction of links with a reciprocal connection, or the relative abundance of cycles of a given length within the network, were also computed to support the idea of a small-world wiring in cortical networks [191,742]. It is worth to notice that in most of the cases the networks are considered as unweighted. However, the description of neural system may be improved by using weighted networks [30,31]. More information on the connection strength is available for some connectivity databases, as that of the $C$. elegans and cat cortex $[730,729,737]$. The interpretation of recent studies within the framework of the small-world behavior has suggested the hypothesis of minimization of connections between brain sites [701-705]. Indeed, some physical models support this optimal wiring principle which minimize the axonal length and the energy requirement of neurons [746-748].

Together with the small-world property, some cortical networks display a multicluster organization which can be related to a scale-free behavior [737]. Moreover, some preliminary studies suggest that the changes observed in the cortical network structure as a consequence of the removal of some nodes are very similar to what is observed in scale-free networks [749]. However, one of the major problem to statistically assess a scale-free behavior in neural connectivities, is the small size of the available databases.

As mentioned above, the functional specialization of local cortical areas are strongly determined by their extrinsic and intrinsic connections [750]. For example, the local organization of connections seems to play an important role in the organization of visual system in the macaque monkey [725,732]. Further, it has been predicted that the variability of the hierarchical model of the visual system can be explained by the fact that connections between such cortical areas never form strong and directed cycles [751].

Using a non-parametric clustering approach, the analysis of the connectivity of cat's visual system suggested that each local connectivity pattern has a unique characteristics [752]. Recently, the set of local connections of cortical areas was proposed to be unique and thus to constitute a connectional fingerprint [750]. Statistical multivariate analysis including the multidimensional scaling and a hierarchical clustering approach [727,729,733-735,753] revealed that local connectivity patterns of each cortical areas are well organized. Further, it has been suggested the existence of families of cortical areas displaying common connectional fingerprints [750].

To study the relationships between local connections and more distributed topological properties of cortical networks, different local connectivity indices have been computed [754]. The so-called connection density, efferent and afferent connections ratio, and the symmetry of connections aimed to quantify the local connectivity of a directed graph. Such measures are related not only to the density of connection per node but to the direction of the links. Using a correlation between the different topological indices and a clustering approach, cortical areas with similar local connectivity patterns have been identified [754]. Such local connectivity patterns are then related to functional contributions of each brain area to the whole cortical network [750,754].

Recently, some families of local patterns in neural connections have been identified by motifs analysis (see Section 2.1.4) $[63,65,66,755]$. Surprisingly, the local organization of neural links significatively displays some motifs as feedforward loops or bi-parallel connections [63,755]. Within the cortical connectivity, a distinction has been made between functional and structural motifs [755]. Structural motifs refer to neural elements connected by anatomical connections pathways, whereas functional motifs consider the possible combinations of nodes and links within the structural motifs. 
Results suggest that cortical networks present a relatively low diversity of motifs, as compared with equivalent random networks, whereas the occurrence and diversity of functional motifs seems to be maximized [755]. Although the interplay between the topology and function of brain motifs remains unknown, their existence in neural networks of different species suggests local organizations with common modes of information processing [445,755].

As a consequence of their clustered topology, the vulnerability of neural networks (the lost of efficiency in the information processing for instance) could therefore be influenced by the inter-cluster connections. The effect of modifications in the connectivity (related to brain lesions) in the propagation of the brain activity was studied by means of a simple model of neural dynamics [702]. As expected, the response of the network to node removals was found to be strongly related to the target node's connectivity. Instead, the variability of the response was higher in brain areas with few connections than in those with a widespread connectivity [756]. Despite the small size of databases, topological changes induced by node and link removals were also studied in neural networks [749,757]. Using local connectivity indices leads to detect vulnerable pathways, i.e. those pathways whose removal induces important damages (quantified here as an increase in the characteristic path length). Such sensitive links were found to be those which connect nodes with low degrees [757].

In the quantification of the brain connections structure, most studies considered only the anatomical connectivity. Only few works have attempted to assess a complex topology from the temporal correlation between brain activities recorded by EEG, MEG or fMRI techniques. The functional connectivity patterns of MEG recordings from healthy patients were found to display a small-world behavior dependant of the frequency band observed [758]. Connectivity patterns were obtained by means of a nonlinear correlation index computed between time series filtered at different frequencies. If the correlation between two sites was higher than a given threshold, a connection was considered as present. Topological indices (characteristic path length and clustering coefficient) were then computed with these unweighted matrices reflecting the presence or absence of a connection. Recently, a similar analysis was performed on fMRI time series [759,760]. An unweighted graph was obtained from the linear correlation matrix by deleting all connections below a certain arbitrary threshold. The connectivity patterns were then used to detect functional clusters related to a stimulus during a task of finger tapping [759]. By means of a similar approach, the analysis of connectivity patterns in fMRI has recently suggested a scale free behavior in brain functional networks [760]. We must notice that correlation-based connectivity patterns lead to undirected graphs. It is therefore expected that the effective connectivity, which describes the causal relationship between neural events, would reveal new topological properties of neural networks. Using functional correlations to assess brain connectivity patterns may present some drawbacks. Firstly, results may depend on the correlation method used. Neural oscillators are essentially complex nonlinear systems, hence a nonlinear character of their interactions is expected [696]. Appropriate statistical methods are therefore necessary to deal with the possible nonlinearities embedded in the time series. Second, as functional interactions are strongly time-dependent, the correlation methods need to capture the fluctuations in the interactions [720]. A natural question concerns the true brain connectivity reflected in the fMRI signals. Two regions clearly connected by the white fiber tracts, for example, will display high functional connectivity, but the inverse is not true [761]. It is worth noticing that the functional connectivity may be strongly biased by partial volume effects such that two spatially adjacent sites display a positive biased cross-correlation. Moreover, functional correlations between some brain areas are very sensitive to slow frequencies $(<0.1 \mathrm{~Hz})$, or to physiological noise sources as the respiratory and cardiac activities [762]. Therefore, in order to assess a significant complex topology in brain connectivity, a careful statistical study would be necessary.

One of the major problems in the statistical analysis of brain connectivity is the small size of the available databases. The use of large surrogate networks is therefore necessary to assess significant relationships between topology and functionality of neural systems. Nevertheless, artificial small-world or scale-free networks are commonly generated by mechanisms [2-4] which are inappropriate descriptors of neural wiring development. A main drawback in some approaches is that they do not take into account the spatial distance as a physical limit for the wiring procedure, and therefore, the correct framework to approach this issue would be that of spatial networks, largely discussed in Section 2.5. The degree of connectivity of pyramidal cells in the rat's neocortex for instance, is tightly linked to the spatial distance to the surrounding cells. Namely, the probability of excitatory and inhibitory connections between pyramidal cells and interneurons decreased only slightly with distance [763]. Recently, synaptic connection strengths were also found to be spatially correlated, i.e. strong connections are more spatially clustered than weak ones forming a skeleton of strong connections [764]. To deal with this problem, a spatial-growth algorithm based on a spatial constraint has been proposed [247,765]. Such a procedure leads to generate surrogate networks with similar topological properties 
as those observed in cortical connectivity patterns. In a different approach, the information related to the strength connections in the cat cortex was transformed into a probabilistic generating function, which was then used to create surrogate connectivity matrices with different wiring schemes (local, long-range or a mixture of both) [743]. As the spatial-growth algorithm, this probabilistic procedure leads to generate graphs which are topologically similar to the cortical networks, but with an arbitrary size.

\subsubsection{Dynamics}

The dynamical interactions between different neural assemblies allows to integrate different sources of information into a coherent brain task [696,706,708,709]. Experimental studies have shown that the firing rate of spontaneously active single neurons in cortical areas strongly depends on the cortical networks in which they are embedded [766]. Brain dynamics would be thus characterized by being simultaneously differentiated in local groups and integrated into a coherent behavior [696,706,708,710,767].

To study the interplay between brain functions and neural connectivity, a measure called complexity was introduced to quantify to what extent functional specialized (local) groups are integrated in a coherent overall (global) dynamics $[710,767,768]$. Differentiation (also called segregation) measures the relative independence of local assemblies, whereas integration refers to the deviation from the independence in large groups of neural elements. Using statistical tools from the information theory (estimators of single and conditional entropies), it has been shown that networks with high complexity are characterized by a coexistence of a high degree of local interactions which are themselves integrated in a coherent dynamics [191,710,742,767-769]. For the sake of simplicity, Gaussian dynamics were used in these studies to mimic spontaneous neural activity. Such linear stochastic process were then used to compute the complexity index on different cortical connectivity matrices. Weights were associated to different links such that all connections were excitatory and such that the variance of signals was kept at a given value [742,767]. Results suggest that cortical networks tend to maximize the interplay between local interactions and a global integration, which is reflected in high complexity values [191,742]. The hypothesis of an optimal organization type local-global in cortical networks was supported by the fact that the complexity values were significantly higher than those obtained in equivalent random networks. Another related measure, called functional cluster index, was applied to real neurophysiological data to identify a functional integration of distributed and organized neural activities [770]. Complexity analysis of different neurophysiological data (PET, fMRI and EEG) supports the hypothesis that some brain states are characterized by an organized emergence of different and sparse local neural dynamics [770,771].

Synchronization through local and global interactions has been proved to be an ubiquitous phenomenon underlying different neural processes in both normal and anomalous brain functions [709]. The study of synchronization has largely been carried out by using neural network models with global [772,773] or local [774,775] couplings. However, real neural networks have been found to display a more sparse connectivity, and the mechanisms of interaction of distinct neural assemblies are strongly related to the degree of randomness in the topology of connections. Computational simulations of hippocampal activity for instance, have shown that for random connectivity patterns, there exist a critical threshold in the degree of connectivity, which rules the overall synchrony. Such minimum number of needed synaptic connections per neuron was indeed found to be independent of the network size [776]. Similarly, the study of networks of integrated and fire oscillators, revealed that random topologies do not display a synchronous behavior if the corresponding graph has a low clustering coefficient [777]. However, if the connectivity per node is increased, random networks may display a coherent and synchronous dynamics. Random connectivity, or random distribution of connection strengths, have thus provided an interesting framework to study different brain dynamics in large neural networks [778-781].

Several studies have suggested that the topology of connections in real neural networks presents a combination of topological properties such that it can be captured neither by regular connectivity models as lattices, nor by random connections. Using a neural network model based on cellular automates, it has been shown that real cortical topologies lead to a propagation of the activity better than in networks with a regular (nearest neighbors) topology, or with equivalent random wirings [702]. Using networks of Hodgkin-Huxley (HH) neurons [782], fast but not coherent oscillatory responses were obtained by means of a random connectivity. On the other hand, regular topologies displayed coherent oscillations but with lower time scales, that can be an obstacle in the information processing of neurons. These behaviors contrast with the small-world topology, which was found to produce both fast and coherent oscillatory responses [426].

Using a network of FitzHugh-Nagumo (FN) neurons [783,784], the frequency of the coherent oscillations was found to be highly modulated by the network topology [785]. In contrast with the HH model, the propagation speed in the 
FN network is not dependent of the wiring structure. The network topology has been found to play a crucial role in other neural collective phenomena as stochastic resonance [786] or coherence resonance [787]. For a certain coupling strength and noise level, an improvement to the stimulus response coherence may be obtained by an increase in the randomness of the network connectivity and a small heterogeneity of the neuron parameters [430,788-790].

In the small-world connectivity, the addition of a few number of shortcuts leads remote neurons to communicate such that local clusters of synchronized activity can be integrated in a global and coherent dynamics [791-793]. However, several works have shown that a large heterogeneity in the number of connections per node may produce a decrease in the synchronization $[777,792,794]$.

Cooperations between local and global interactions was shown to be relevant in cooperative phenomena as feature binding where spatially distant stimuli can produce a synchronous response of different neural assemblies [709,795]. In contrast with regular local connectivity patterns (which are not able to display a global synchronous behavior in response to local stimuli), for certain degree of randomness in the wiring such stimuli may produce a highly coherent pattern of activity [796]. Within the framework of learning of artificial neural networks, it has recently been shown that a small-world connectivity may yield a learning error and learning time lower than those obtained with random or regular connectivity patterns [797].

Another phenomenon that has been largely studied is the formation of associative memories. The aim of an associative memory system is to store a set of patterns in such a way that when a new pattern is presented, the system responds with a stable state corresponding to the most similar pattern stored [798]. Although real brain networks have a sparser connectivity than traditional fully connected neural models, they also display an organization of local modules or clusters. Different studies have shown that sparse connectivities may modify the capacity of storage and retrieval of patterns in different models of associative memory [799-801]. On the other hand, memory systems based on intermodular connections were found to allow a good retrieval, more resilient to local damage [802].

In order to combine both properties, interconnected modularity and some degree of sparse random connectivity, some associative memory models (based on Hopfield models and Hebbian learning rules [798]) have introduced a small-world topology in the architecture of the networks. The performance of such networks exhibits a phase transition at a certain degree of randomness which implies that a fraction of shortcuts may enhance the efficiency of the network for the storage and retrieval of patterns while keeping the same wiring costs [803-807].

The effect of the degree distribution in the performance of associative models has been studied by means of Hopfield networks with scale-free topologies [804,806-809]. Although the performance of memory systems with a scale-free connectivity is better than that with equivalent random connections, the existence of highly connected nodes makes the recognition of patterns to be very partial (limited to the hubs) [804]. Indeed, the performance of the network is sensitive to both the local organization of connections (related to the clustering coefficient) and the heterogeneity of the distribution. The best performances in terms of storage and retrieval were obtained for networks with low clustering coefficient [807], or in networks with a uniform degree distribution [806,808,809].

Some brain diseases, as epilepsy, are the result of an abnormal and, some times, abrupt synchronization of a large number of neural populations. This synchronization is an essential feature of the generation and propagation of epileptic activity. However, despite considerable progress in understanding the physiological processes underlying epileptic dynamics, the network mechanisms involved in the generation, maintenance and propagation of the epileptic disorders are still unknown. To study the role of connectivity in epileptic dynamics, different neural models were implemented with a small-world connectivity. These realistic simulations were based on networks of excitatory neurons of the hippocampal circuits. Results showed that a diversity of neural dynamics as spontaneous, bursts, or seizure-like activities can be obtained by changing the coupling (synapse) strength, the connectivity degree and/or the degree of randomness in the connections [810]. The network can display a bursting regime for instance, if the synaptic efficiency is increased, as well as the connectivity degree. However, increasing the heterogeneity in the degree distribution produces a decrease in the synchronization. In the brain cortex there exist two basic neuron types: excitatory neurons and inhibitory interneurons. Although different configurations of inhibitory and excitatory neurons can give rise to coherent oscillatory dynamics, the simplest one is formed by a homogeneous network of inhibitory interneurons [444,811-813]. Interneurons connectivity is known to be highly local but there exists a small number of long-range interneurons which allow to connect distinct cortical regions. Such synaptic shortcuts have recently been related to a small-world behavior [444]. The relevance of this property has been studied in recent works which considered networks of inhibitory neurons with a small-world topology $[444,814]$. Results confirmed the idea that a small degree of randomness in the wiring of a local connectivity may highly improve the synchronization of neural dynamics. Fig. 6.11 illustrate this finding. In a related work, the 


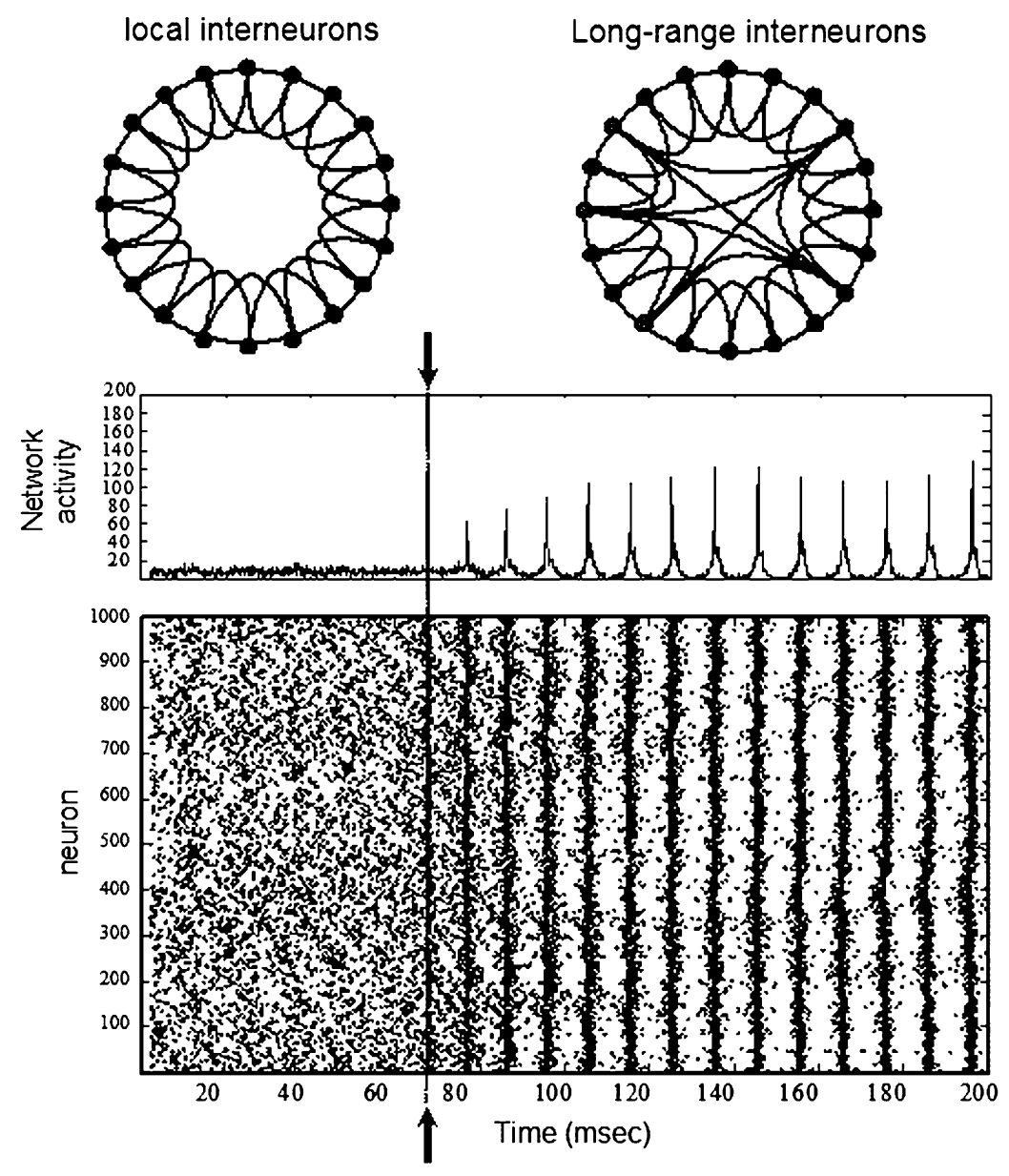

Fig. 6.11. Enhancement of synchronization in a network of inhibitory interneurons by the addition of a small number of long-range interneurons. Upper panel: example of connectivity schemes. Middle panel: spike raster of 1000 neurons. Bottom panel: average population firing rate. The red arrow indicates the instant when the shortcuts are added. Figure taken from Ref. [814]. Courtesy of M. Le van Quyen.

analysis of cortical neural cultures has lead to study the interplay between connectivity and frequency of synchronized neural discharges. Instead of using the physical information, a correlation between the neural activities was used to estimate the degree distribution of cultured networks. Results suggested that the bursting frequency of synchronized firing was strongly related to the node degree [815].

\subsubsection{Open questions}

Even if the study of brain connectivity has already provided some clues to understanding some brain functions, several aspects remain still unclear:

- The small-world behavior observed in cortical connections might be the result of a wiring optimization procedure under spatial constraints, obtained in the course of evolution of the brain circuits. However, the observed connectivity might be also the result of an optimization for the information processing. New realistic models of neural connectivity, including both anatomical (spatial constraints) and functional (exchange and integration of information) costs should be developed.

- Larger data sets, currently unavailable, would be required to assess whether neuroanatomical networks are scale-free. Moreover, the standard mechanisms to generate scale-free networks are inappropriate for neural wiring development.

- The presence of motifs is a relevant feature in the structure of cortical networks. However, the role of motifs in brain dynamics remain unclear. Future computational simulations might provide some clues for this issue [816]. 
- In most of the literature only symmetric and unweighted connection matrices have been considered. However it is reasonable that weighted and/or asymmetric couplings might play a role in neural dynamics [817].

- Most of connectivity schemes used in neural modelling do not take into account the spatial distance in the wiring procedure. In large neural networks, spatially distant connections (shortcuts) imply a higher energetic cost [701-705]. More realistic models including spatial constraints should be therefore considered in the future [247,765].

- Some current works on neural dynamics consider only electrical couplings between the elements of the networks. However, neurons can communicate among them through chemical or electrical synapses. Moreover, both mechanisms were found to play complementary roles in the coherence of rhythms displayed by the whole network [818]. Similarly, the interaction of inhibitory and excitatory neurons may give rise to different properties in the collective activities [813].

- In most of the cases a time-independent connectivity is considered. Conversely, brain connectivity is often modulated by changes in the properties of neurons or through synaptic plasticity [707,773]. Long-term memory is, for instance, currently related to changes in the strengths of connections between cortical neurons. Experimental evidence suggests that learning could induce changes in the wiring scheme [819]. Together to weight changes, the wiring plasticity could provide some boost in storage capacity. Computational models are therefore required to study how neural dynamics can be affected by a time-varying connectivity.

- Synchronization has been found to be enhanced by a small-world wiring structure. This finding has suggested a plausible paradigm to explain the emergence of some neural phenomena as epileptic seizures, characterized by a sudden and strong synchronization. Unfortunately, there is presently no conceptual or computational works to understand the role of connectivity in the control of these synchronized states [820]. In any case, a reliable approach to neural mechanisms of brain diseases will be possible only through the mixing of theoretical and experimental studies [814].

\section{Other topics}

In this final chapter we consider three topics that have recently attracted a large interest in the scientific community. We first discuss the problem of partitioning large graphs into their community structures, i.e. tightly connected subgraphs. This is basically a structural problem. We then review the recent advancements in finding reliable and fast ways for navigation and searching in a complex network. The chapter ends with a discussion of adaptive and dynamical wirings.

\subsection{Algorithms for finding community structures}

Community structures are a typical feature of social networks, where some of the individuals can be part of a tightly connected group or of a closed social elite, others can be completely isolated, while some others may act as bridges between groups. The differences in the way the individuals are embedded in the structure of groups within the network have important consequences on the behavior they are likely to practice. In fact, social subgroups often have their own norms, orientations and subcultures, sometimes running counter to the official culture, and are the most important source of a person's identity. Social analysts were the first to formalize the idea of communities, to devise mathematical measures of the number and cohesion of communities, and to develop methods to identify the subgroups of individuals within a network [18,19]. Community structures are also an important property of other complex networks. For instance, tightly connected groups of nodes in the World Wide Web often correspond to pages on common topics, while communities in cellular [646] and genetic networks [821] are related to functional modules $[62,822]$. Consequently, finding the communities within a network is a powerful tool for understanding the structure and the functioning of the network, and its growth mechanisms. In Section 2.1.5 we have discussed several different definitions of community. Here we focus instead on the methods to find communities. The general aim of such methods is to find meaningful divisions into groups by investigating the structural properties of the whole graph. The two major aspects concern with algorithmic issues and with the way to measure how good the obtained division is.

We first discuss two traditional approaches, namely spectral partitioning [823-828] and hierarchical clustering $[18,19]$, that work quit reliably for specific types of problems, but perform poorly in more general cases [829]. We 
then review some methods proposed in the physics community in the last few years. The general discussion will be for undirected, non-valued graphs, although most of the methods presented can be easily generalized.

\subsubsection{Spectral graph partitioning}

Graph partitioning is a classical problem in computer science and consists in finding the division of the vertex set $V$ into two nearly equally sized vertex subsets $V_{1}^{*}$ and $V_{2}^{*}$, with the minimum number of edges running between the two subsets; formally $V_{1}^{*}$ and $V_{2}^{*}$ must be such that $\operatorname{cut}\left(V_{1}^{*}, V_{2}^{*}\right)=\min _{V_{1}, V_{2}} \operatorname{cut}\left(V_{1}, V_{2}\right)$, where $\operatorname{cut}\left(V_{1}, V_{2}\right)=\sum_{i \in V_{1}, j \in V_{2}} a_{i j}$. Graph partitioning finds many practical applications such as load balancing in parallel computation, circuit partitioning and telephone network design, and is known to be a NP-complete problem [830].

Many efficient heuristic methods have been proposed over the years to cope with this intrinsic difficulty, in particular those based on spectral methods. Spectral graph partitioning was introduced in the early 1970s [823-825] and popularized in 1990 [826]. Spectral partitioning generally gives better global solutions than other heuristic methods, as the Kernighan-Lin [831] and the Fiduccia-Mattheyses [832] algorithms, that search in the local vicinity of given initial partitionings and have a tendency to get stuck in local minima. The early methods were based on the partitioning properties of the eigenvectors of the adjacency matrix $\mathscr{A}$ [828]. More direct methods are based on the combinatorial Laplacian matrix $\Lambda$ and the Normal matrix $\mathscr{N}$, defined in Section 2.1.6. Here we focus mainly on the spectrum of $\Lambda$, although similar results can be obtained from the normal matrix [828].

The Laplacian spectrum has many attractive properties $[833,834]$ that make it very useful for partitioning large sparse arrays. As discussed in Section 2.1.6, $\Lambda$ has the lowest eigenvalue $\lambda_{1}=0$, with eigenvector $\mathbf{v}_{1}=(1,1, \ldots, 1)$. When the graph consists of $c$ components, i.e. it can be perfectly separated into $c$ non-overlapping communities, the multiplicity of the null eigenvalue is equal to the number of components $c$ [825]. On the other hand, when the graph has an apparent, though not perfect, community structure with $c$ communities, there will be only one eigenvalue equal to 0 , $c-1$ eigenvalues slightly larger than 0 , and the remaining eigenvalues lying a gap away from 0 . Fiedler first suggested in 1975 that the eigenvector $\mathbf{v}_{2}$ associated to the second smallest eigenvalue $\lambda_{2}$ (the so-called Fiedler eigenvector) could be used to find an approximate solution to the graph partitioning problem, based on the signs of the components of $\mathbf{v}_{2}$. The first subgraph is made by vertices with a positive component, the second by vertices with a negative component [826,835], with values of $\lambda_{2}$ closer to 0 corresponding to better splits. This is the spectral bisection method that works quite well and is fast if the graph admits a good splitting into two communities. In fact, while in general it takes a time $\mathcal{O}\left(N^{3}\right)$ to find the eigenvectors of a $N \times N$ matrix, the calculation of only a few eigenvectors is less computationally demanding. The current method of choice for finding a few eigenvectors of very large sparse matrices is the Lanczos method [828,836]: in particular the calculation of the second eigenvector can be accomplished in a time that goes as $K /\left(\lambda_{3}-\lambda_{2}\right)$ [829]. This means that the method is linear in $N$, although in practice the method can be rather slow when $\lambda_{3} \sim \lambda_{2}$.

Following Ref. [829] we show how the method works on the karate club network of Zachary [837], a test case in which the community structures are known. The network represents the friendship relationships between the members of a karate club and was obtained from data collected by an anthropologist, Wayne Zachary, over a 2-year period of observations. The graph consists of 34 nodes and 78 edges and is depicted in Fig. 7.1. During the period of study, due to contrasts between a teacher (labelled as node 1) and the administrator of the club (node 34), the club was observed to split into two smaller groups of size 16 and 18. In Fig. 7.1 the members of the two groups are respectively indicated by circles and squares. The presence of a certain polarization into two different groups is already evident from a visual inspection of the graph. The two groups obtained by the spectral bisection method are shown in grey and white colors in Fig. 7.1a. The division is almost perfect with only node 3 classified in the wrong community. For a recent application of the spectral bisection method to the Internet and to electric power grids see Rosato et al. [77,838]. The bisection method works extremely well when the most natural partition is really that into two communities, as in the case of the karate club. If this is not the case, the division into a larger number of communities can be obtained by iterative bisection, although the disadvantage is that repeated bisections are not guaranteed to reach the best partition. Moreover, there are no indications on when the bisection process should terminate, unless one has some prior information on the number of expected communities.

\subsubsection{Hierarchical clustering}

A class of algorithms that work much better when there is no prior knowledge on the number of communities is the hierarchical clustering analysis used in social networks analysis $[18,19]$. The term clustering has here a different 


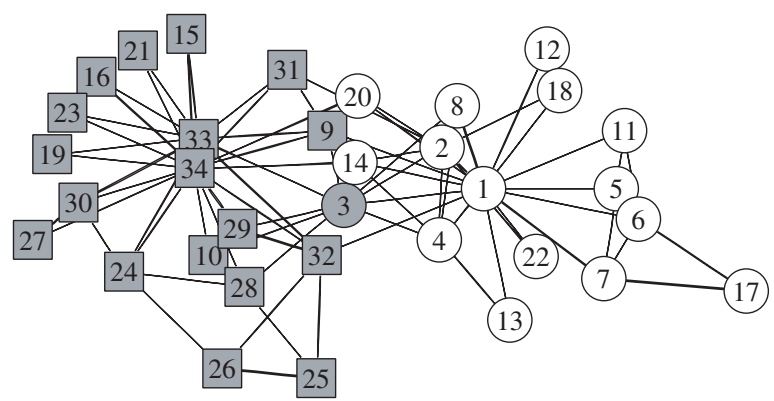

(a)

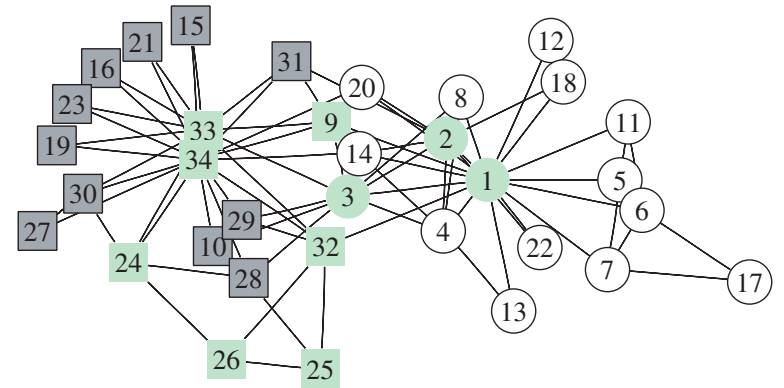

(b)

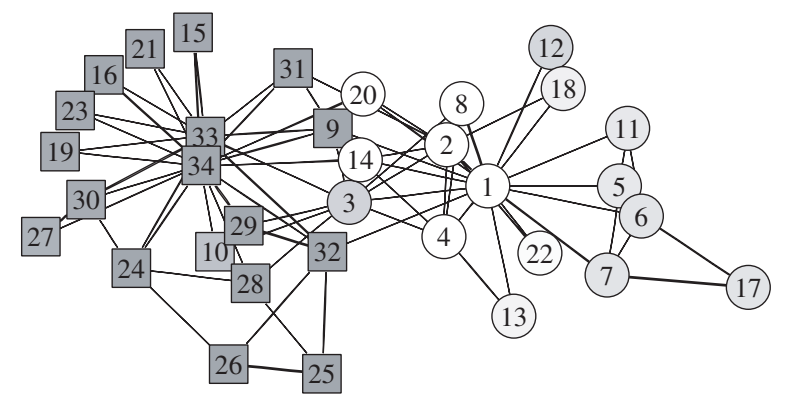

(c)

Fig. 7.1. Finding community structures in the karate club network of Zachary. The numbered vertices of the network represent the members of the club, while the edges represent friendships, as determined by the observation of the interactions [837]. The two groups into which the club split during the course of the study are indicated by the squares and circles, while the dark grey and white show the divisions of the network found by (a) the spectral bisection algorithm of Section 7.1.1, (b) the hierarchical clustering method of Section 7.1.2 and (c) the Monte Carlo sampled version of the algorithm of Girvan and Newman proposed by Tyler et al. and discussed in Section 7.1.4. In (b) the lightly shaded vertices are those not assigned by the algorithm to either of the two principal communities. In (c) shades intermediate between the dark grey and white indicate ambiguously assigned vertices that fall in one community or the other, or neither, on different runs of the algorithm. Reprinted figure with permission from [829]. (C) 2004 by the European Physical Society.

meaning from that of Section 2.1.3. In fact, the general aim of cluster analysis is to organizing $N$ objects into groups (clusters), such that objects within each cluster are more closely related to one another than objects assigned to different clusters. Of course, the central concept in cluster analysis is the notion of distance or similarity/dissimilarity between the individual objects being clustered (see, Ref. [839] as an example of how to define a distance in the case in which the objects to be clustered are time series). In the case we want to find the community structures in a graph, the objects to be clustered are the nodes of the graph and the similarity between two nodes is extracted from the network structure. This can be done in different ways as we will discuss momentarily.

In hierarchical clustering, the data are not partitioned into a particular cluster in a single step. Rather, a series of partitions takes place, which may run from a single cluster containing all objects to $N$ clusters each containing a single object. Hierarchical clustering is subdivided into agglomerative methods, which proceed by series of fusions of the $N$ objects into groups, and divisive methods, which separate $N$ objects successively into finer groupings. Agglomerative techniques are more commonly used. Given a set of $N$ items to be clustered, and an $N \times N$ distance matrix $\mathscr{D}$ (or similarity matrix $\mathscr{R}$ ), the basic process of agglomerative hierarchical clustering, as defined by Johnson in 1967 [840], is the following [841]:

1. Start by assigning each item to a cluster, so that to have $N$ clusters, each containing just one item. Let the distances (similarities) between the clusters be the same as the distances (similarities) between the items they contain.

2. Find the closest (most similar) pair of clusters and merge them into a single cluster.

3. Compute distances (similarities) between the new cluster and each of the old clusters.

4. Repeat steps 2 and 3 until all items are clustered into a single cluster of size N. 


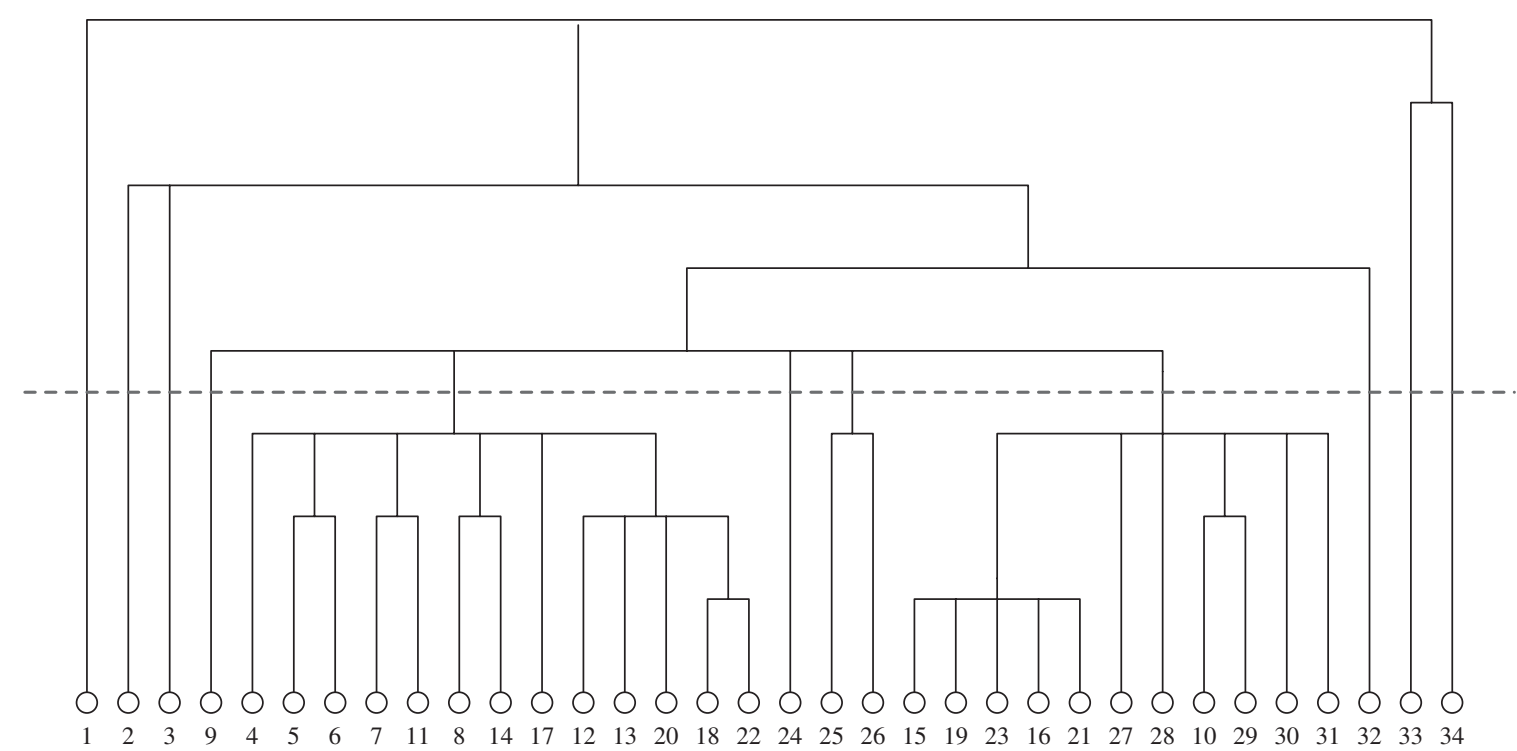

Fig. 7.2. The hierarchical tree (dendrogram) depicting the results (from bottom to top) of a single linkage agglomerative hierarchical clustering of the karate club network based on Euclidean distance defined in Eq. (7.1). A cross-section of the tree at any level will give the communities at that level. The cross-section indicated by the dotted line corresponds to the community division shown in Fig. 7.1b. The vertical height of the branching points in the tree is indicative only of the order in which the joins between vertices take place. Note that the heights of some joins coincide, indicating that the vertices joined at that level have identical similarities. Reprinted figure with permission from [829]. (C) 2004 by the European Physical Society.

The resulting hierarchical structure to clusters can be represented by dendrograms, or hierarchical trees, as the one reported in Fig. 7.2, showing the clusters produced at each step of the agglomeration (or subdivision). Step 3 can be done in different ways, which is what distinguishes between different methods as the single-linkage, the complete-linkage and the average-linkage. In the single-linkage clustering, the distance between two clusters is equal to the shortest distance from any member of one cluster to any member of the other cluster. If the data consist of similarities, the similarity between two clusters is equal to the greatest similarity from any member of one cluster to any member of the other cluster. In the complete-linkage, the distance between two clusters is defined as the longest distance from any member of one cluster to any member of the other cluster. In average-link clustering, the distance is equal to the average distance from any member of one cluster to any member of the other cluster.

When the objects to be clustered are the nodes of a graph, the similarity is defined in terms of structural equivalence. Structural equivalence is a key concept in social networks introduced and defined by Lorrain and White in 1971 [842]. Basically, two nodes of the graph are structural equivalent if they have the same pattern of relationships with all the other nodes: for instance two individuals in a friendship network are structurally equivalent if they have the same friends. There are different ways to measure structural similarity, and such measures are used as a basis for identifying sets of nodes that are structural similar to one another and distinct from nodes in other sets. Structurally equivalent nodes have identical entries in their corresponding rows (columns) of the adjacency matrix. The use of Euclidean distance as a measure of structural equivalence was first proposed by Burt in Ref. [843]. For nodes $i$ and $j$ this is the distance between rows (or columns) $i$ and $j$ of the adjacency matrix [18]:

$$
D_{i j}=\sqrt{\sum_{k=1, k \neq i, j}^{N}\left(a_{i k}-a_{j k}\right)^{2}} .
$$

If nodes $i$ and $j$ are precisely structurally equivalent, then the entries in their respective rows of $\mathscr{A}$ will be identical and thus $D_{i j}=0$. Euclidean distance has the properties of a distance metric: (1) $D_{i j} \geqslant 0 \forall i$, j; (2) $D_{i i}=0 \forall i$; (3) $D_{i j}=D_{j i} \forall i, j$. Euclidean distances are computed between all pairs of nodes in the graph and are the entries of the matrix $\mathscr{D}$ to be used in the hierarchical clustering algorithms. 
An alternative measure of structural equivalence is based on the correlation between rows (or columns) of the adjacency matrix. The Pearson correlation coefficients are defined as

$$
R_{i j}=\frac{\sum_{k=1}^{N}\left(a_{i k}-\bar{a}_{i .}\right)\left(a_{j k}-\bar{a}_{j .}\right)}{\sqrt{\sum_{k=1}^{N}\left(a_{i k}-\bar{a}_{i .}\right)^{2}} \sqrt{\sum_{k=1}^{N}\left(a_{j k}-\bar{a}_{j}\right)^{2}}},
$$

where by $\bar{a}_{i}$. we denote the mean of the values in row $i$. The coefficients $R_{i j}$ range from -1 to 1 (if two nodes are perfectly structurally equivalent, then the correlation between their respective rows in A will be equal to 1), and are used to construct a similarity matrix $\mathscr{R}$. For an extension of Eqs. (7.1) and (7.2) to valued and directed graphs, and also to multiple relations, see Ref. [18].

As an example of how the method works in practice, in Fig. 7.2 we show the dendrogram resulting from the application to the karate club network of Section 7.1.1. Following Ref. [829], we consider a single linkage agglomerative hierarchical clustering based on Euclidean distance. It can be shown that the overall run-time of the algorithm scales as $\mathcal{O}\left(N^{2} \log N\right)$ on a sparse graph [829]. Although hierarchical clustering methods do not require any prior knowledge of the size and number of groups, they do not tell which is the best division of the network, i.e. at which level the tree should be considered. The horizontal dotted line reported in Fig. 7.2 has been chosen as the one corresponding to a division most closely to the known division of the club. The resulting community division is shown in Fig. 7.1b. The method classifies correctly a substantial number of nodes. It does not however assign some relevant nodes as 1, 33, and 34 to any major group. Such a problem, which is typical of hierarchical clustering, makes this method unsatisfactory for the analysis of many large real networks.

\subsubsection{The algorithm by Girvan and Newman}

The lack of a method which works reliably for general cases has led, in the last few years, to the introduction of a series of new methods, initiated by the seminal work by Girvan and Newman [673].

Girvan and Newman have proposed an iterative divisive method based in finding and removing progressively the edges with the largest betweenness, until the network breaks up into components [51,673]. Since the edges that lie between communities are expected to be those with the highest betweenness, by removing them recursively a good separation of the network into its communities can be found. The general form of the Girvan Newman (GN) algorithm is the following:

1. Calculate the betweenness score for each of the edges.

2. Remove the edge with the highest score.

3. Perform an analysis of the network's components.

4. Go back to point 1 until all the edges are removed and the system breaks up into $\mathrm{N}$ non-connected nodes.

The recalculation of the edge betweenness every time after an edge has been removed appears to be an important aspect of the algorithm [51]. The outcome of the algorithm is a dendrogram similar to the one shown in Fig. 7.2, with the difference that now each branching corresponds to a splitting event, and the algorithm proceeds from top (one component of $N$ nodes) to bottom ( $N$ single nodes) of the figure.

The performance of the algorithm has been tested on artificial random modular networks, i.e. computer generated random graphs constructed in such a way to have a well defined community structure. All graphs have the same number of nodes, 128, and the same number of edges, 1024. The nodes are divided into four groups and the edges are labelled according to whether they connect members of the same group or not. The mixing between the classes is introduced by tuning the average number of edges connecting nodes belonging to different classes. From a generic vertex of the graph we have on average $z_{\text {in }}$ edges which join it to other vertices of its group and $z_{\text {out }}$ edges connecting it to vertices of the other groups. The two numbers are not independent, since $z_{\text {in }}+z_{\text {out }}=16$. The final aim of the test is to consider how many vertices are correctly classified by the algorithm as a function of $z_{\text {out }}$. As an example of how the algorithm works on a graph of this type we show in Fig. 7.3 the dendrogram obtained. For illustration purposes a smaller network, with 64 nodes and 8 edges per node, has been considered. Here, $z_{\text {in }}=6$ and $z_{\text {out }}=8-z_{\text {in }}=2$, i.e. the network has a strong community structure. The algorithm produces a hierarchy of subdivisions of the network: from a single component to $N$ isolated nodes, going from left to right in the figure. To know which of the divisions is the best one for a given network, i.e. where to cut the hierarchical tree, one can use the modularity $Q$, a quantity introduced in Ref. [51] and defined in 


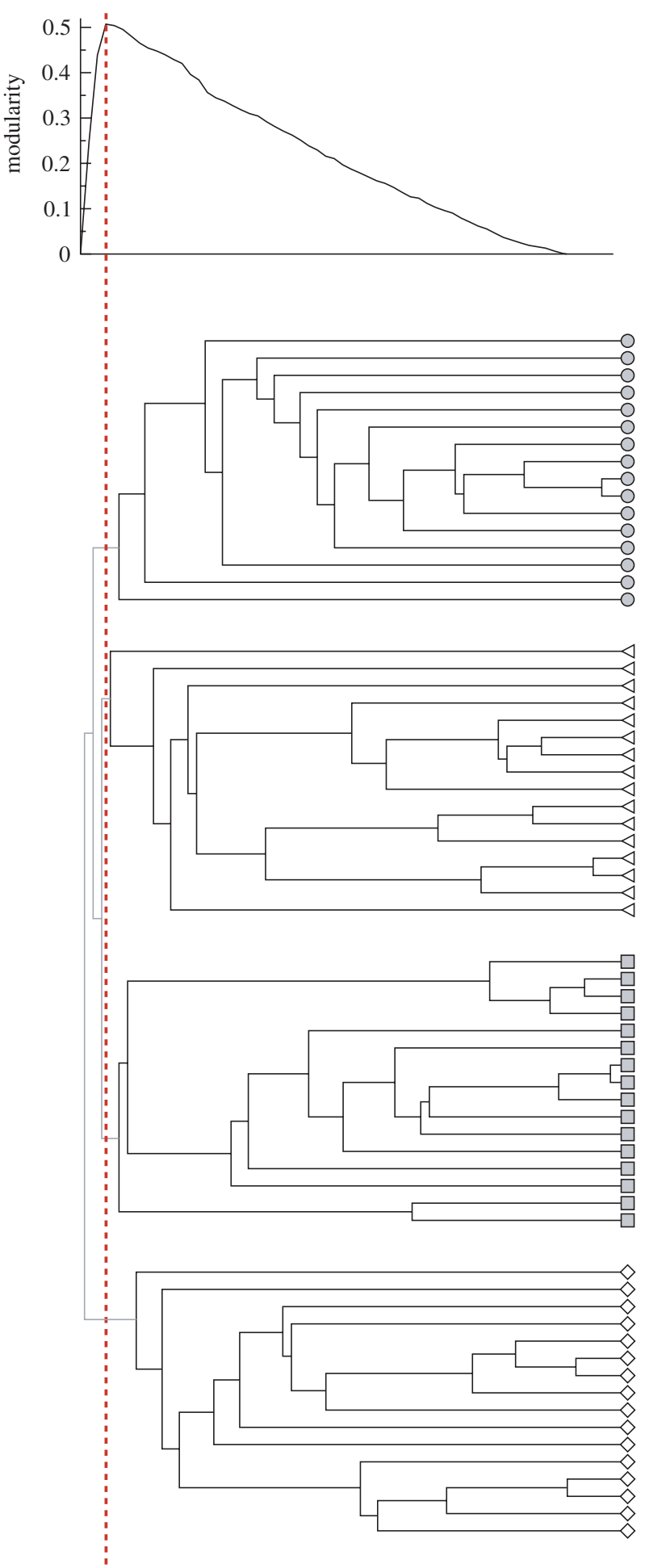

Fig. 7.3. Modularity $Q$ (top panel) and dendrogram (bottom panel) obtained by the application of the GN algorithm to a computer generated modular random graph with 64 vertices and 256 edges. The random graph has been generated, as described in the text, by dividing the nodes into 4 groups of 16 nodes each (respectively empty circles, full circles, triangles and squares) and by considering $z_{\text {in }}=6, z_{\text {out }}=2$. The peak in the modularity (dotted line) corresponds to a perfect identification of the 4 communities. 


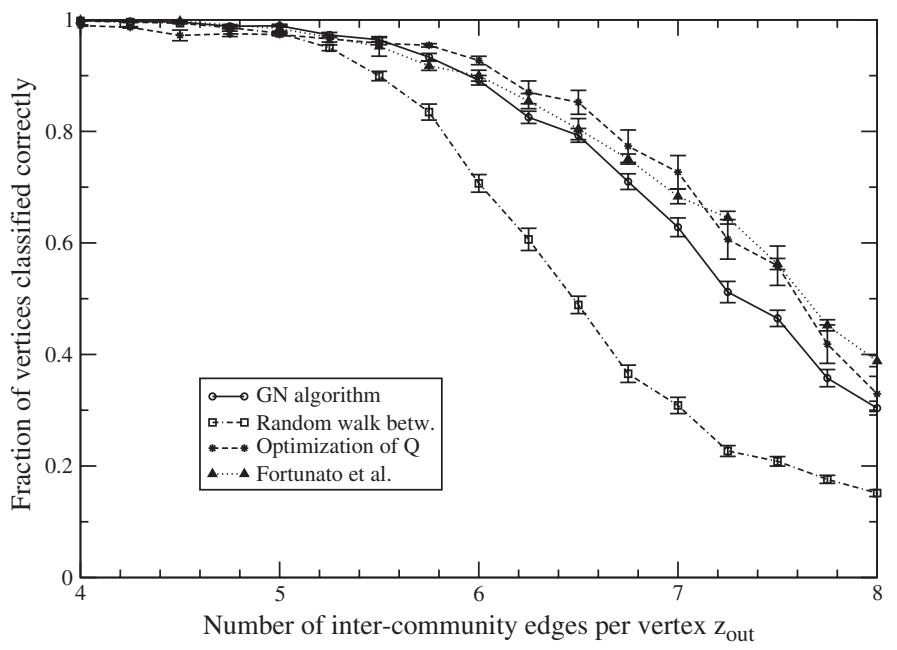

Fig. 7.4. Average fraction of correctly identified vertices as a function of $z_{\text {out }}$. Each point represents an average over 100-500 computer generated modular random graphs with 128 vertices and 1024 edges. The results of the GN algorithm are compared with those obtained by using the random betweenness, the optimization of $Q$, and the Fortunato et al. algorithm (See Section 7.1.4).

the following way. Let us suppose that we want to test the goodness of a subdivision of $G$ in $n$ given communities. We expect that a good split is obtained if most of the edges fall inside the communities, with comparatively few edges joining the communities to each other. Then one introduces a $n \times n$ symmetric matrix $E$ whose element $e_{i j}$ is the fraction of all edges in the network that link vertices in community $i$ to vertices in community $j$. The trace of this matrix $\operatorname{Tr} E=\sum_{i} e_{i i}$ is the fraction of edges in the network that connect vertices in the same community, while the row (or column) sums $a_{i}=\sum_{j} e_{i j}$ give the fraction of edges that connect to vertices in community $i$. If the network is such that the probability to have an edge between two sites is the same regardless of their eventual belonging to the same community, one would have $e_{i j}=a_{i} a_{j}$. Finally the modularity is defined as

$$
Q=\sum_{i}\left(e_{i i}-a_{i}^{2}\right)=\operatorname{Tr} E-\left\|E^{2}\right\|,
$$

where $\left\|E^{2}\right\|$ indicates the sum of the elements of the matrix $E^{2}$. The modularity measures the degree of correlation between the probability of having an edge joining two sites and the fact that the sites belong to the same community. Values approaching $Q=1$, which is the maximum, indicate a strong community structure; conversely, $Q=0$ for a random graph with no community structure. Local peaks in the modularity during the progress of the algorithm indicate particularly good divisions of the graph. The modularity $Q$ corresponding to the groups determined after each split is reported in top panel of Fig. 7.3. In the case of a random modular graph with $z_{\text {in }}=6$ and $z_{\text {out }}=2$ the modularity exhibits a single peak, that exactly corresponds to the splitting of the network into the four groups. This means that the algorithm succeeds in identifying the four classes. The height of the peak is around 0.5 , which is a typical value for highly clustered networks. The performance of the GN algorithm can be tested by considering many different realizations of the same graph with a given value of $z_{\text {out }}$ and checking how many vertices are correctly classified in each case at the maximum value of $Q$. The average fraction of nodes which end up in their natural group as a function of $z_{\text {out }}$, for modular random graphs with 128 nodes and 1024 edges, is reported in Fig. 7.4. The performance of the algorithm is excellent with more than $90 \%$ elements being classified correctly up to $z_{\text {out }}=6$. The results of the GN on the karate club are illustrated in the dendrogram of Fig. 7.5. The modularity exhibits two relative maxima (dotted lines) corresponding to divisions into two and five communities respectively. The first maximum, with a relatively high value of $Q$, indicates that there is a strong natural division of the network into two communities, although the split into five communities gets a higher modularity score. Moreover the two groups obtained match closely the two factions observed experimentally: only one node, node 3 , is misclassified.

The GN method has been employed by different authors in several studies. The algorithm has produced a series of very good results in a variety of networks [51,673], including networks of e-mail messages [844], human [533,845,846] 


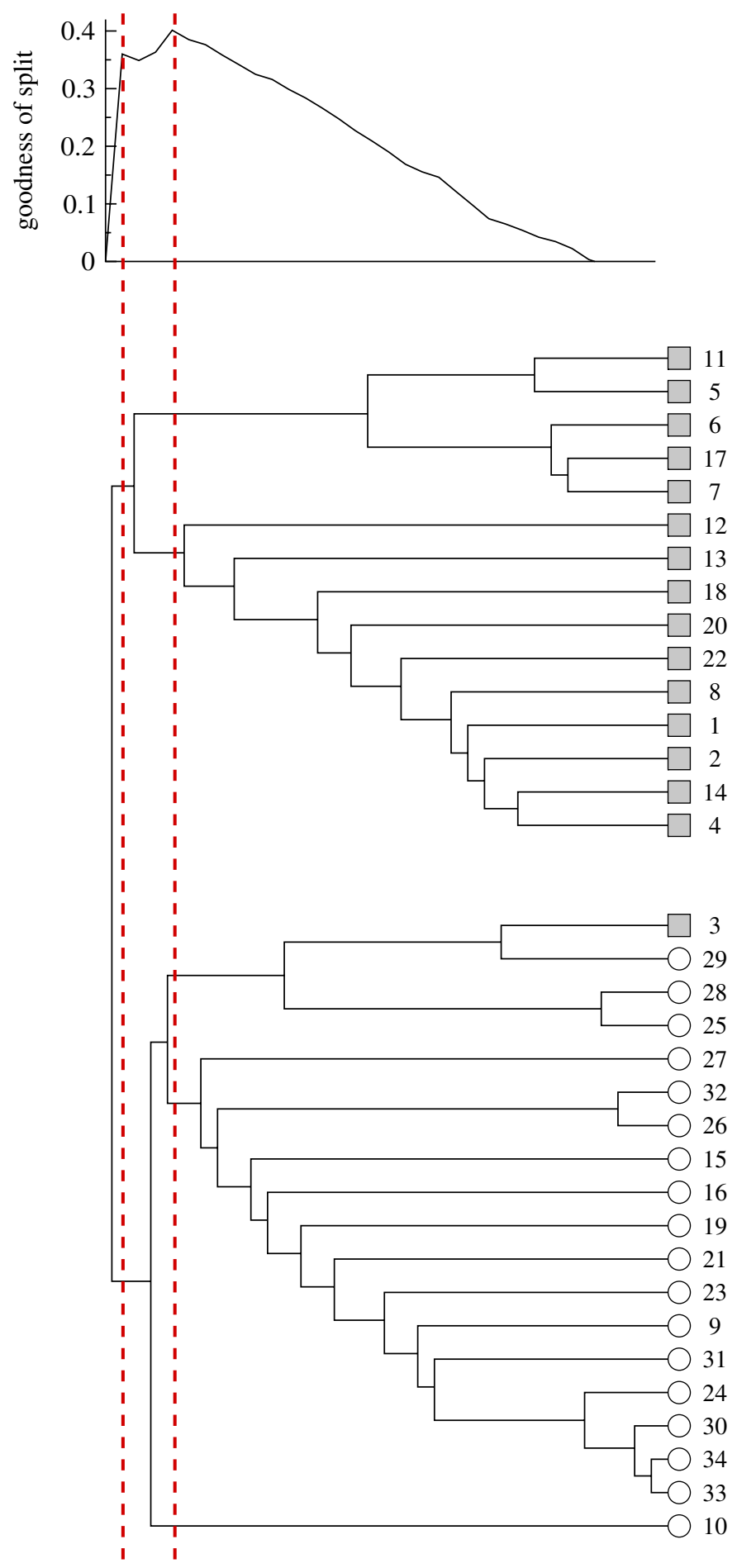

Fig. 7.5. Modularity $Q$ (top panel) and dendrogram (bottom panel) obtained by the application of the GN algorithm to the karate club network The modularity has two maxima corresponding respectively to (i) a split into two communities, which matches closely the real split of the club (only node 3 is incorrectly classified), and (ii) a split into five communities. Reprinted figure with permission from Ref. [51]. (C) 2004 by the American Physical Society. 
and animal [847] social networks, networks of collaborations between scientists [51] and musicians [532], metabolic networks [646,848] and gene networks [849]. For a straightforward extension of the algorithm to weighted networks, based on the mapping of a graph with integer weights into a multigraph with a number of links between two nodes equal to the weight of the link, see Ref. [850]. The only problem with the GN method, is that its computational demand is rather high. Evaluating the betweenness for all edges requires a time of the order of $K N$ [40,41]. Consequently, the GN algorithm runs in $\mathcal{O}\left(K^{2} N\right)$ time on an arbitrary graph with $K$ edges and $N$ vertices, or $\mathcal{O}\left(N^{3}\right)$ time on a sparse graph. This restricts the applications to networks of at most a few thousands of vertices with current hardware facilities.

\subsubsection{Variations and extensions of the GN algorithm}

Following the work of Ref. [673], a series of algorithms based on the idea of iteratively removing edges with high centrality score have been proposed. Such methods use different measures of edge centrality, as the random-walk betweenness, the current-flow betweenness [51], and the information centrality [851]. Random-walk betweenness considers random walks connecting all couples of nodes instead of the shortest paths [51]. Random walks have also been used in other algorithms for finding communities, either to quantify the similarities-dissimilarities between nearestneighboring nodes [852-854] (see Section 7.1.7), or to drive the collective exploration of the graph in agent-based approaches [855]. The current-flow betweenness is defined by considering an electric circuit associated to the graph, and by using Kirchoff's laws. Despite the different definitions, it has been proven that the current-flow betweenness coincides with the random-walk betweenness [51]. Algorithms based on random walk and current-flow betweenness run to completion in a time $\mathcal{O}\left(N^{4}\right)$.

In Ref. [851], Fortunato et al. have proposed an algorithm to identify directly the edges that, once removed, mostly disrupt the network's ability in exchanging information among the nodes. As a measure of centrality this algorithm adopts the information centrality $C^{I}$ [37], that is a quantity based on the concept of efficient propagation of information over the network $[30,31]$. The information centrality has revealed an interesting quantity to characterize the centrality of the nodes of a network $[34,37,47,528]$, and it gives results different from those based on the betweenness centrality. The information centrality $C_{k}^{I}$ of the edge $k$ is defined as the relative drop in the network efficiency caused by the removal of the edge from $G$ :

$$
C_{k}^{I}=\frac{\Delta E}{E}=\frac{E(G)-E\left(G_{k}^{\prime}\right)}{E(G)}, \quad k=1, \ldots, K .
$$

Here $G_{k}^{\prime}$ is the graph with $N$ nodes and $K-1$ edges obtained by removing the edge $k$ from $G$, and $E(G)$ is the efficiency of the graph $G$ [30,31]. The results of this algorithm for the modular random graphs are shown in Fig. 7.4. For $6 \leqslant z_{\text {out }} \leqslant 8$, where the communities are very mixed and hardly detectable, the algorithm performs slightly better than the GN algorithm and the modularity-based algorithm of Section 7.1.5. The algorithm runs to completion in time $\mathcal{O}\left(K^{3} N\right)$, or time $\mathcal{O}\left(N^{4}\right)$ on a sparse graph, and for its high computational time it can be used only for graphs with a few hundred of nodes.

While being of pedagogical interest, the variations discussed above make greater computational demands and seem to give no much better results than the original GN algorithm based on shortest-path betweenness. In Ref. [844] Tyler et al. have introduced a stochastic element into the GN algorithm that allows a node to belong to different communities and improves the speed of the calculation at the cost of a reduction in accuracy. The algorithm is based on a Monte Carlo estimate of edge betweenness that tends to the true betweenness as the size of the sample becomes large: instead of considering the contributions to edge betweenness coming from all possible paths, only a random sample of them is chosen. Tyler et al. have shown that good results can be obtained with reasonably small sample sizes, which could potentially offer substantial speed improvements over the original algorithm. Moreover, by repeating the calculation many times with different random numbers, vertices whose community assignment is ambiguous, are sometimes put in one community and sometimes in another, and one can make an estimate of how strongly each node belongs to each community. In Fig. 7.1c we showed, following Ref. [829], how the method works on the karate club network. The figure is obtained by running the algorithm 20 times and then averaging the results. One community, the one on the left in the figure, is unambiguously determined. This is not true for the second community. There are some peripheral nodes (in light grey) whose community assignment is less strong. Such nodes, as for instance node 3 , fall in one community or the other, or neither, on different runs of the algorithm. Another modification of the GN algorithm, that allows nodes 
to be placed in several communities and provides a quantitative estimate of how strongly each node belongs to each community, can be found in Ref. [821].

In Ref. [71], Radicchi et al. have proposed a variation to the GN algorithm based on the counts of short cycles. The idea is very simple and interesting: the edge betweenness is a quantity whose value depends on the properties of the whole graph; an algorithm much faster than the GN can then be obtained by using local (instead of global) quantities for the measurements of the edge centrality. Radicchi et al. have proposed to consider the edge-clustering coefficient, defined, in analogy with the usual node clustering coefficient, as the number of triangles to which a given edge belongs, divided by the number of possible triangles that can be built given the degrees of the nodes. More formally, the clustering coefficient of the edge connecting node $i$ to node $j$ is given by:

$$
C_{i, j}^{(3)}=\frac{z_{i, j}^{(3)}+1}{\min \left[\left(k_{i}-1\right),\left(k_{j}-1\right)\right]},
$$

where $z_{i, j}^{(3)}$ is the number of triangles built on that edge and $\min \left[\left(k_{i}-1\right),\left(k_{j}-1\right)\right]$ is the maximal possible number of them. The constant +1 is included at the numerator to avoid problems that can arise when the number of triangles is equal to zero, and guarantees that nodes with only one connection are not considered as isolated communities by the algorithm, since for their unique edge $C_{i, j}^{(3)}$ is infinite. The algorithm works exactly as the GN, with the only difference that now, at every step, the removed edges are those with the smallest value of $C_{i, j}$. In fact, edges connecting nodes in different groups belong to few triangles (or no triangles at all) and tend to have small values of $C_{i, j}^{(3)}$. The computational time is $\mathcal{O}\left(K^{2}\right)$ on an arbitrary graph, or $\mathcal{O}\left(N^{2}\right)$ on a sparse graph. Higher order clustering coefficients $C_{i, j}^{(g)}$, with $g>3$, can be defined in a similar way as in formula (7.5), by considering the number of cyclic structures of order $g$ the edge $(i, j)$ belongs to, divided by the number of possible cyclic structures of order $g$ that can be built given the degrees of the nodes. By considering increasing values of the order $g$, one can smoothly interpolate between a local and a non-local algorithm.

\subsubsection{Fast methods based on the modularity}

In order to deal with large networks, for which some of the previous algorithms turn out to be not viable, Newman has developed in Ref. [856] a fast method directly based on the optimization of the modularity of formula (7.3). Since the exhaustive search of all possible divisions would take a time exponential in $N$, the method is a "greedy" optimization based on the iterative agglomeration of small communities. Starting with $N$ communities, each containing a single node, the communities are repeatedly joined together in pairs, by choosing at each step the join that results in the greatest increase (or smallest decrease) in $Q$. The joining of a pair of communities between which there are no edges can never result in a change in $Q$. Consequently, only pairs between which there are edges need to be considered, of which at any time there will at most $K$. The change in $Q$ upon joining two communities $i$ and $j$ is given by $\Delta Q=e_{i j}+e_{j i}-2 a_{i} a_{j}=2\left(e_{i j}-a_{i} a_{j}\right)$. Following a join, some of the matrix elements $e_{i j}$ must be updated by adding together the rows and columns corresponding to the joined communities, which takes a time $\mathcal{O}(N)$. Thus, each step of the algorithm takes a time $\mathcal{O}(K+N)$, and the entire dendrogram can be constructed in time $\mathcal{O}((K+N) N)$, or $\mathcal{O}\left(N^{2}\right)$ on a sparse graph, allowing to analyze networks of size up to $N=50000$ in less than 1 hour of CPU time on a personal computer [856]. The method appears to work well both in test cases and in real-world situations and can be trivially generalized to weighted networks [856].

In [857] Clauset et al. have proposed a new algorithm that performs the same greedy optimization as the algorithm of [856], and therefore gives identical results for the communities found. However, by exploiting some shortcuts in the optimization problem and using more sophisticated data structures, the algorithm runs far more quickly, in time $\mathcal{O}(K D \log N)$ where $D$ is the depth of the "dendrogram" describing the network's community structure. For sparse networks and networks that have a hierarchical structure with communities at many scales (i.e, $D \sim \log N)$ the algorithm has essentially linear running time, $\mathcal{O}\left(N \log ^{2} N\right)$. This is not merely a technical advance but has substantial practical implications, as it allows to study networks with million of nodes in reasonable run times.

\subsubsection{Other methods based on spectral analysis}

The study of the eigenvector associated with the second smallest eigenvalue of $\Lambda$, discussed in Section 7.1.1, is of practical use only when a clear partition into two parts exists, which is rarely the case. A possible approach is the 


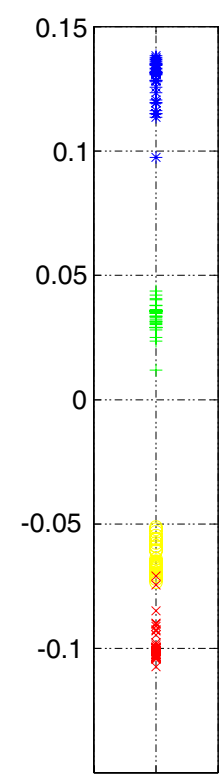

(a)

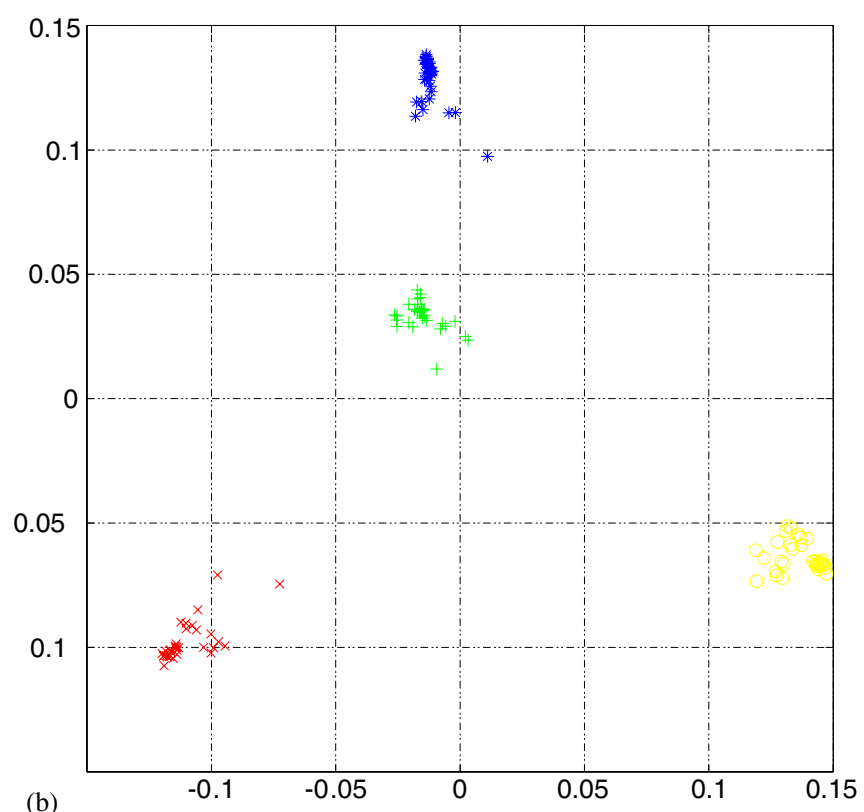

Fig. 7.6. (a) Components of the first non-trivial eigenvector $\mathbf{v}_{2}$ for a computer-generated network with four communities (see main text). Two communities are clearly identified while the other two overlap. (b) All communities can be clearly identified when the components of $\mathbf{v}_{3}$ are plotted versus those of $\mathbf{v}_{2}$. Figure taken from Ref. [859].

recursive spectral bisection: use Fiedler eigenvector to divide the graph into two subgraphs, find the Fiedler eigenvector of each sub-graph, and continue recursively until the desired number or size of sub-graphs is reached. In most common occurrences, however, the number of nodes is large and the separation between the different communities is rather smooth so that the communities cannot be simply detected by looking iteratively at the first non-trivial eigenvector. A better possibility is that of combining information from the first few non-trivial eigenvectors of $\Lambda$ (or $\mathscr{N}$ ). This idea has been explored recently by Capocci et al. [858] and by Donetti et al. [859]. As an example, in Fig. 7.6a, we show the components of the first non-trivial eigenvector of $\Lambda, \mathbf{v}_{2}$, for a computer-generated graph including four communities, each composed by 32 nodes (created as in Section 7.1.3). As expected, the nodes can be split into two groups according to the sign of the respective components. Moreover, we notice that the components of $\mathbf{v}_{2}$ assume similar values on node belonging to the same community, so that, in principle, it is somehow possible to find all the four communities from a more accurate inspection of the spectrum. The group structure in the figure is clear, even if the two communities at the bottom are very near to each other and some nodes could be misclassified. In cases with a number of inter-group connections larger than here the communities become more entangled, and the prospective of extracting clear-cut subdivisions using this type of one-dimensional plot worsens. This difficulty can be circumvented by taking into account more eigenvectors and enlarging the projection-space. This is illustrated in Fig. 7.6b, where the nodes of the same graph are plotted using the components on the first two non-trivial eigenvectors as coordinates. Simple eye-inspection shows that all communities are distinctly separated now. Actually, by using three eigenvectors some further improvement in inter-community separation can be obtained.

The method proposed by Donetti et al. generalizes this idea. Each vertex in the graph is represented by a point in a $D$-dimensional space in which the coordinates are given by its projections on the first $D$ non-trivial eigenvectors. Then, a quantitative measure of similarity between two nodes of the graph is extracted either from the Euclidean or from the angular distance between the respective points in the $D$-dimensional space. The angular distance is defined as the angle between the vectors joining the origin of the $D$-dimensional space with the two points under consideration, and in the study cases reported in Ref. [859] gives better results than the Euclidean one.

Communities are extracted by standard agglomerative hierarchical clustering methods based on such distance matrix. The modularity $Q$ is used as a way to estimate the quality of a given splitting. The division giving the maximum value of the modularity $Q$ is taken as the output of the algorithm for a fixed $D$. Then, also $D$ is allowed to vary, from 1 to 
some arbitrary value, and the best $D$ is selected as that giving splitting with the maximum modularity. Although the maximum number of eigenvectors to be computed in order to find the one generating the maximum modularity is not known a priori, the author have shown that in most of the practical cases $Q$ reaches a rapid convergence to the optimal value as a function of $D$ [859].

In an alternative approach, Capocci et al. [858] proposed to extract the community structure from the correlations between the same components in different eigenvectors. In fact, components corresponding to nodes belonging to the same communities are still strongly correlated taking, in each eigenvector, similar values among themselves. Thus, a natural way to identify communities in an automatic manner, is by measuring the correlation:

$$
R_{i j}=\frac{\left\langle v_{i} v_{j}\right\rangle-\left\langle v_{i}\right\rangle\left\langle v_{j}\right\rangle}{\left[\left(\left\langle v_{i}^{2}\right\rangle-\left\langle v_{i}\right\rangle^{2}\right)\left(\left\langle v_{j}^{2}\right\rangle-\left\langle v_{j}\right\rangle^{2}\right)\right]^{\frac{1}{2}}},
$$

where the average is over the first $D$ non-trivial eigenvectors. The quantity $R_{i j}$ measures the community closeness between node $i$ and $j$. Though the performance may be improved by averaging over more and more eigenvectors, with increasing computational effort, Capocci et al. have found that indeed a small number of eigenvectors $D$ suffices to identify the community to which nodes belong, even in large networks [858]. The calculation of eigenvectors is the slowest part of the two previous algorithms. The running time strongly depends on $D$, the number of eigenvectors one needs to compute by the Lanczos method [828,836]. A roughly estimate gives $\mathcal{O}(D N)$.

A different spectral method, based on the slowest decaying modes of a diffusion process taking place on the network, can be found in Ref. [619].

\subsubsection{Other algorithms}

Various other algorithms, based on the most different ideas can be found in the literature. For instance, Wu and Huberman have proposed a method based on the notion of voltage drops across the network [849]. The graph is transformed into an electric circuit by associating a unit resistance to each of its edges. Two nodes of the graph are given a fixed potential difference. The method does not make use of current flows to define edge betweenness as in Ref. [51] (see Section 7.1.4). Instead, communities are defined from the resulting voltage values of each node. The method is based on an approximate iterative algorithm that allows to solve the Kirchoff's equations for node voltages in time $\mathcal{O}(N+K)$, i.e. in linear time (exact methods involve inversion of the Laplacian matrix and take a time $\mathcal{O}\left(N^{3}\right)$ in the worst case $[51,849])$. The method works quite well as a graph bisection, and can be extended to graphs with more than two communities, although with all the drawbacks typical of bisection methods.

Reichardt and Bornholdt have proposed in Ref. [860] a method based on a q-state Potts model associated to the graph. In such a case, communities are found to coincide with the domains of equal spin value in the minima of a modified Potts spin-glass Hamiltonian. An interesting feature of this method is that no prior knowledge of the number of communities is required. Moreover, by comparing global and local minima of the Hamiltonian it is possible to detect overlapping communities and to quantify the association of nodes to multiple communities.

Zhou have proposed a hierarchical clustering algorithm (see Section 7.1.2) based on random walks [852-854]. First of all a distance $d_{i j}$ from vertex $i$ to vertex $j$ is defined as the average number of steps needed for a Brownian particle to move from vertex $i$ to vertex $j$ [852]. This is done by considering that, at each vertex (say $k$ ), the Brownian particle will jump in the next step to a nearest-neighboring vertex (say $l$ ) with probability $P_{k l}=a_{k l} / \sum_{m=1}^{N} a_{k m}$. The distance matrix thus defined is asymmetric and can be calculated by solving $N$ linear-algebraic equations [852]. Taking any vertex $i$ as the origin of the network, then the set $\left\{d_{i 1}, \ldots, d_{i, i-1}, d_{i, i+1}, \ldots, d_{i N}\right\}$ measures how far all the other vertices are located from the origin. Therefore, it is actually a perspective of the whole network with vertex $i$ being the viewpoint. Consequently, a dissimilarity index $D_{i j}$ between two nearest-neighbors vertices $i$ and $j$ can be defined as:

$$
D_{i j}=\frac{\sqrt{\sum_{k \neq i, j}^{N}\left[d_{i k}-d_{j k}\right]^{2}}}{(N-2)} .
$$

If two nearest-neighboring vertices $i$ and $j$ belong to the same community, then the average distance $d_{i k}$ from $i$ to any another vertex $k(k \neq i, j)$ will be quite similar to the average distance $d_{j k}$ from $j$ to $k$. Thus, $D_{i j}$ will be small if $i$ and $j$ belong to the same community and large if they belong to different communities. A hierarchical clustering algorithm (see Section 7.1.2) is then worked out on the dissimilarity matrix $\mathscr{D}$, and each of the resulting communities is characterized by an upper and a lower dissimilarity threshold [853]. 
(a)
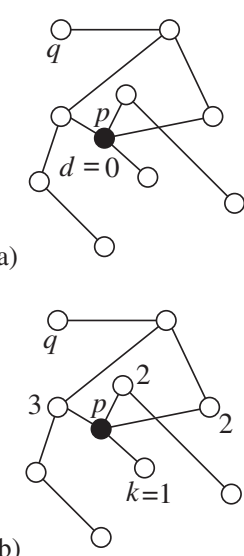
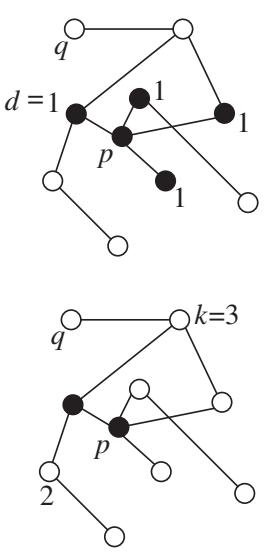
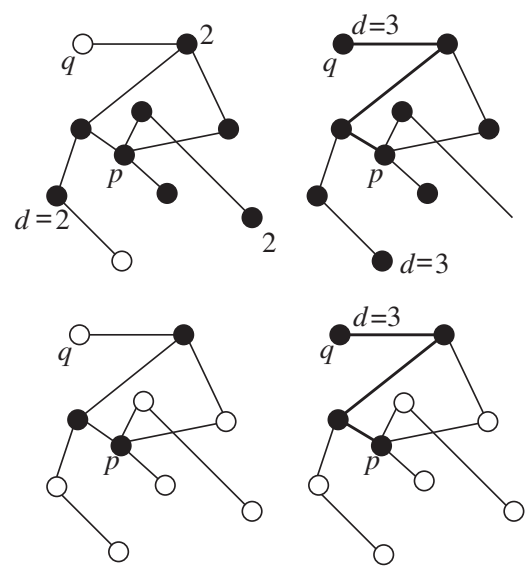

Fig. 7.7. Schematic diagram of (a) the breadth-first search algorithm and (b) the maximum degree strategy for searching from node $p$ to node $q$. In all cases $d$ denotes the step number of the algorithm. Reprinted figure with permission from Ref. [861]. C) 2002 by the American Physical Society.

\subsection{Navigation and searching}

Milgram's pioneering experiment and related works discussed in Section 2.2.1 elucidate two interesting aspects of social networks [89,90]: (1) relatively short paths exist in large size networks; (2) individuals are able to find short paths even in the absence of a global knowledge of the network. While the former feature is recovered in the various models described in Section 2.3, the latter property is not yet clearly understood. The ability to navigate and search for shortest paths in a network without knowledge on its whole topological structure is a relevant issue, not only in social systems, but also in optimization of information finding from the World Wide Web, traffic way-finding in a city, transport of information packets on the Internet, or diffusion of signaling molecules in a biological cell.

In this section, we review some of the studies on the subject. Two main approaches have been followed: one is based on finding the best strategy for constructing a path to a target node from an initial node by using local or geometric information; the second aims at assessing the most efficient network structure for path searching, once the way of finding a path is fixed.

\subsubsection{Searching with local information}

We start by discussing the breadth-first search algorithm [40], also known as burning algorithm [862], that is one of the most used methods to find the shortest path between a source $p$ and a target vertex $q$ in a network [20-22]. The method is based on the following iterative algorithm. At the initial step $(d=0)$, an agent is put in the source vertex $p$. The agent inspects the neighborhood $\mathscr{N}_{p}$ of the source vertex. If the target vertex $q$ is not among those neighbors, then at $d=1$ agents are spread to all those elements of $\mathscr{N}_{p}$ that have not been reached by any other agent in the past times. In the successive iterations $(d>1)$, each new agent repeats the same procedure of verifying and spreading, up to the moment in which the target vertex is attained by a given agent. By back-tracing the route of that agent, one is then able to reconstruct a path from the source to the target. This procedure is depicted in Fig. 7.7a. The method allows for the exact individuation of the shortest path, but it requires the presence of multi-agents and, in general, it needs $O(N)$ computation times to construct the path.

We now consider strategies where only one agent is used to construct paths between two vertices. Noh and Rieger have investigated random walks on complex networks and derived an exact expression for the mean first passage time (MFPT) between two nodes [863]. This allows to introduce a random walk centrality $C$ for each node. Among the set of all possible random walk motions between two nodes, the walk to the node corresponding to a larger value of $C$ is faster than the others, and, in a given time interval, nodes with larger $C$ values are visited more frequently by initially uniformly distributed random walkers. Analytical and numerical results show that the information does not distribute uniformly in heterogeneous networks, rather it is centralized in a specific set of nodes possessing large values of $C$, as indicated in Fig. 7.8. Fig. 7.8 also shows that, for a BA scale-free network, the degree of a node is directly related to its centrality. As a consequence, if a target node has a small degree, the probability to reach that target by a random walk will be very small. 


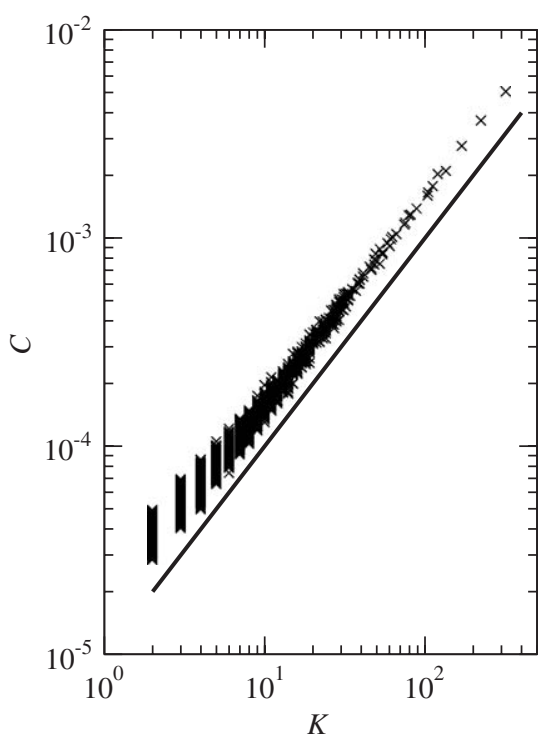

Fig. 7.8. Centrality coefficient $C$ vs. node degree $K$ for BA scale-free network, in a double logarithmic plot. The straight line is a guide for the eyes. Reprinted figure with permission from Ref. [863]. (C) 2002 by the American Physical Society.

In order to design efficient methods for searching a specific file in peer-to-peer networks, Adamic et al. have proposed the so-called maximum degree strategy, that is based on the assumption that a vertex has information on its neighbors' degree [6,864]. The procedure is schematically sketched in Fig. 7.7b. The agent always moves to the neighbor having highest degree. If the highest-degree vertex has been already visited by the agent in the past, then the second highest is chosen, and so forth. In this way the agent rapidly reaches the high-degree vertices. Since in a highly heterogeneous network, as the Internet, the highest-degree nodes are connected to a significant fraction of all nodes in the network, the agent need only a few steps to find a node that is a neighbor of the target $q$. An analytical estimate of the search cost $s$, defined as the number of steps until approximately the whole network is revealed, can be obtained for networks with a power-law degree distribution. In particular, in the scale-free model by Aiello et al. [132], discussed in Section 2.3.4, the maximum degree $k_{\max }$ (the cutoff in the power-law degree distribution) defined by $\mathrm{NP}\left(k_{\max }\right)=1$, i.e. as the degree above which there is on average less than one node in the graph, scales as $N^{1 / \gamma}$, with $\gamma$ being the exponent of the power law. For such a model, when $2<\gamma<3$, one obtains $s \sim N^{2-4 / \gamma}$ for the maximum degree strategy. This is smaller than the search cost $s \sim N^{3-6 / \gamma}$ expected for an agent following a random walk [864]. The maximum degree searching algorithm has been tested numerically on real networks and on graphs created by the configuration model [864] and the BA model [861]. The measured searching times are in reasonable agreement with the analytical predictions above mentioned. Kim et al. have compared the average path length of BA scale-free networks as actually measured by different searching strategies such as random walk, maximum degree search, and the preferential choice strategy, i.e. a strategy in which the node with the larger degree has the higher probability to be chosen [861]. Although the standard average path length $L$ is usually defined as in formula 2.5 in terms of the shortest path lengths, in practice finding the shortest path between two nodes requires a global information, which is often not available. It is then useful to define a generalized average path length $L_{s}$ which depends on the lengths of the actual paths found by the searching strategy adopted. The maximum degree strategy produces a generalized average path length that scales with $\log N$ (in analogy with what happens with the breadth-first search method), while $L_{S}$ obtained by the random walk strategy and preferential strategy, scale as a power of the network size.

In the WS model the position of the nodes has an intrinsic ring geometry independently on the network topology. Moura et al. have shown that searching methods exploiting geometric information are successful in constructing short path lengths in such networks [865]. In particular, they have considered a searching strategy in which the next node is selected in the set of neighboring nodes as the one having the closest distance $d$ to the target. The distance $d$ is taken to be the geometric distance along the ring between the two nodes. The cost of such searching procedure includes the looking up time $L_{s}^{\mathrm{LUT}}$, that is the time needed to inspect the neighborhood of the present node, and the search time $L_{s}$ (the average number of steps to reach a desired node). Notice that $L_{s} \geqslant L$, where $L$ is the average shortest path length. 


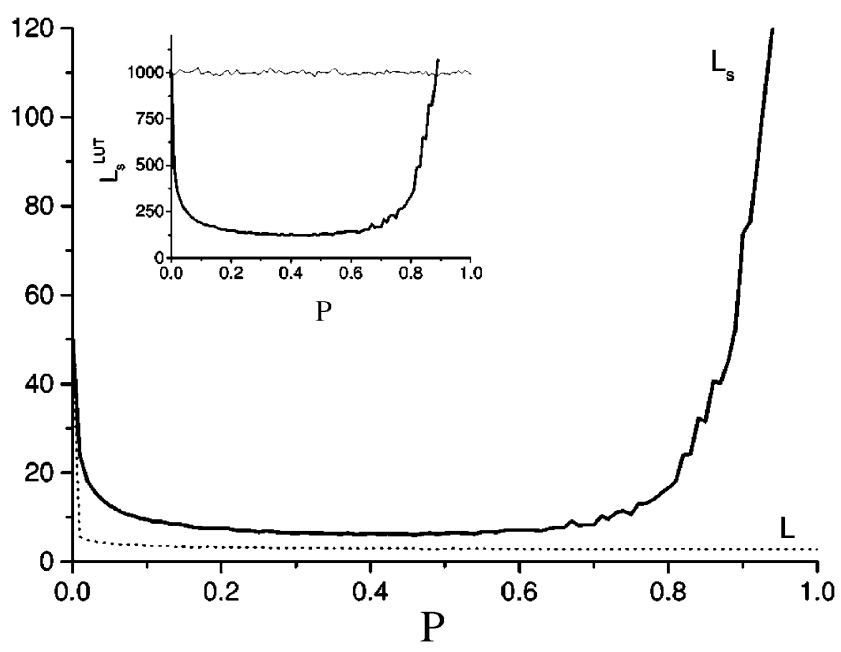

Fig. 7.9. Search time $L_{S}$ (solid line), and average shortest path length $L$ (dashed line), as a function of the rewiring probability $p$ for a WS small-world network with $N=2000$ nodes and $K=10000$ links. The inset shows $L_{S}^{\text {LUT }}$ for the search method adopted in Ref. [865] (thick line) and for the breadth-first search method (thin line). Reprinted figure with permission from Ref. [865]. (C) 2003 by the American Physical Society.

Fig. 7.9 shows the cost of the searching procedure as a function of the rewiring probability $p$. For regular networks ( $p=0$ ), due to the absence of long-range links, both the shortest path $L$ and $L_{s}$ are large. In the limit $p=1$, the distance $d$ is no longer useful to reach the target node, and the search will jump from node to node in a random way, taking a long time to find the target, like a random walk in ER random graphs. Similarly to $L, L_{s}$ reaches the minimum in the intermediate region corresponding to the small-world behavior. The same happens for the looking up time $L_{s}^{\mathrm{LUT}}$, that at each step of the searching is proportional to the number of neighbors of the node currently visited. In particular, it is possible to show with analytical arguments that in the small-world regime $L_{s}$ scales as $N^{1 / D(D+1)}$, where $N$ is the number of nodes and $D$ is the dimension of the underlying lattice. This means that the average search time increases very slowly with the network size, in comparison with regular and random networks, and also with respect to a strategy based on random walk.

\subsubsection{Network navigability}

In the previous section, we have discussed efficient searching methods that do not require knowledge on global topology. We now consider the complementary question of finding the network structure that optimizes a specific search algorithm. Kleinberg was the first to show that efficient navigability is a fundamental property of only some small-world structures [235,236]. He considered a variant to the WS small-world model in which shortcuts are added between pairs of sites on a $2 D$-lattice with a probability $r^{-\alpha}$, where $r$ is the distance between the sites and $\alpha$ is a tunable parameter. Then, he adopted a greedy searching algorithm in which the agents spread to those nodes that are as close as possible to the target (with respect to a lattice distance metrics). Kleinberg proved the existence of a lower bound for the delivery time $s$, defined as the mean number of steps needed to find a randomly chosen target on the network. For $\alpha \neq 2$ the delivery time satisfies $s \geqslant c N^{\beta}$, where $\beta=(2-\alpha) / 3$ for $0 \leqslant \alpha<2$ and $\beta=(\alpha-2) /(\alpha-1)$ for $\alpha>2$, and where $c$ is independent of $N$. Thus the best performance of the greedy algorithm is when $\alpha$ is close to 2 . In particular, when $\alpha=2, s$ is bounded by a function proportional to $(\log N)^{2}$. The results generalize to networks embedded in $D$-dimensional lattices, with the optimal performance of the greedy searching occurring at $\alpha=D$. Recently, Zhu and Huang studied the phenomenon in WS small-world networks with ring symmetry [866].

In a searching experiment on a heterogeneous network, one can imagine that the hubs contribute a sort of "funneling effect" [40], that is for most of the source nodes the bulk of the paths between them and other nodes in the network go through just one or two of their neighbors (those with the highest degree). However, in the electronic replica of the Milgram's experiment carried on by Dodds et al. [92], it was shown that there is only a little dependence of the navigation properties on the hubs. Instead, "social identities" were suggested to play a key role. Social identities are sets of characteristics attributed to individuals such as their occupation or their geographical location. Individuals navigating a social network, in fact, look for common features between their neighbors and the target. Watts et al. proposed an 
alternative to Kleinberg's model in which the individuals are hierarchically grouped into categories, according to their social identities [867]. These categories are grouped into super-categories, and so forth, thus creating a tree-like hierarchy of organization that defines a social distance between any two people, given by the height of the lowest level in the tree at which the two are connected. A network is then constructed by connecting two individuals with a probability which is larger for shorter social distances, and it is assumed to decay off exponentially with longer social distances. The greedy algorithm for communicating a message to a target person specifies that, at each time step, the message passes to that neighbor of the current holder who has the shortest social distance to the target. Like in the case of Kleinberg, the ability to search and find specific targets was found to depend not only on the short network distances, but also on the existence of links constructed with probabilities that decay exponentially with social distances.

Rosvall et al. have proposed to characterize the ease or difficulty of navigation in different networks, in terms of the information needed to locate specific addresses [272]. Suppose that an agent on node $p$ wants to locate through the shortest path (or if there are several, through the degenerate shortest paths) a node $q$ somewhere else in a connected network. When starting at node $p$, the agent has to find the right exit link, in the direction towards target $q$. The information value in knowing one particular exit channel is $\log _{2} k_{p}$, where $k_{p}$ is the degree of node $p$. At the subsequent node $j$ along the shortest path to the target, the number of questions to ask to find the right exit link is reduced to $\log _{2}\left(k_{j}-1\right)$, since the incoming link is known. This means that the total information value of knowing any one of the degenerate paths between $p$ and $q$ is given by

$$
S(p \rightarrow q)=-\log _{2}\left(\sum_{\{\operatorname{path}(p, q)\}} \frac{1}{k_{p}} \prod_{j \in \operatorname{path}(p, q)} \frac{1}{k_{j}-1}\right)
$$

where the sum runs over the set $\{\operatorname{path}(p, q)\}$ of degenerate shortest paths between $p$ and $q$. The authors have investigated the search information $S$ for a number of networks, finding that one needs more information to orient in real than in randomized networks (with the same degree distribution of the real networks) [272]. Moreover, the difference $\Delta S(l)=S(l)-S_{\text {rand }}(l)$ between the average search information for nodes separated by $l$ links in the real networks, $S(l)$, and the same quantity in the randomized counterparts, $S_{\text {rand }}(l)$, is positive and increasing for large distances $l$. This indicates that essentially all the contribution to the excess of $S$ with respect to $S_{\text {rand }}$ comes from large distances. For some real networks, as, for example the Internet at the autonomous system level, $\Delta S(l)$ is even negative at distances shorter than a certain horizon $l_{\text {horizon, }}$ which implies that these real networks are organized to optimize the search at these short distances. Thus, local communication is favored, whereas communication for $l>l_{\text {horizon }}$ is disfavored. A similar behavior is also found in some modelling topologies, as for instance in structured modular networks. Models of hierarchical networks, on the other hand, do worse than random networks on all scales [868].

Guimerà et al. have studied the optimal network structure related with the problem of avoiding congestion effects that arise when parallel searches are performed [333]. For a single search problem the optimal network is clearly a highly polarized starlike structure. This structure is indeed very simple and efficient in terms of searchability, since the average number of steps to find a given node is always bounded, independently of the size of the system. However, the polarized starlike structure becomes inefficient when many search processes coexist in the network, due to the limited capacity of the central node. In Ref. [333] the case was made of a node that can receive an infinite number of incoming packets (and store the packets in a queue), but it is limited on the number of packets that can be sent within a given time interval. Studies on congestion models of network traffic [331] have highlighted that, for low values of packets creation rate $\rho$ the system reaches a steady state in which the total number of floating packets in the network $N(t)$ fluctuates around a finite value. As $\rho$ increases, the system undergoes a continuous phase transition to a congested phase in which $N(t) \propto t$, that is, packets accumulate in the network (for more details see Section 3.2.2). In Ref. [333] it is analytically shown that, for low values of $\rho$, the average load of the network $\langle N(t)\rangle$ is proportional to $\rho N \bar{d}$, where $\bar{d}$ is the average search cost(number of steps) of a packet, while when $\rho$ approaches $\rho_{\mathrm{c}},\langle N(t)\rangle$ is inversely proportional to $1-\rho B^{*} /(N-1)$, where $B^{*}$ is the maximum of the effective betweenness $B_{j}$, which is given by the total number of packets passing through the node $j$ when all possible pathways are considered for all possible pairs of vertices. Therefore, in order to minimize the cost of the whole network in the case of small $\rho$, the network structure should possess small path lengths (as e.g. a starlike structure). Conversely, in the case of higher $\rho$, the betweenness should be minimized. This means that only two classes of networks can be considered as optimal: starlike configurations when the number of parallel searches is small, or homogeneous configurations when the number of parallel searches is large. 


\subsection{Adaptive and dynamical wirings}

Most of the results that have been described so far refer to cases where the wiring topology is static, i.e. it is fixed, or grown, once forever, and the dynamical processes are pertinent to the interactions among elements induced by such static connection schemes. Another possibility is to consider the networks themselves as dynamical entities. This means that the topology is allowed to evolve and adapt in time, driven by some external action, or by the action of the internal elements, or following specific predetermined evolving rules. This step forward is clearly motivated by the need of suitably modelling specific cases, such as genetic regulatory networks [687], ecosystems [869], financial markets [310,870], mutations and evolutions in social and biological phenomena [871], as well as to properly describe a series of technologically relevant problems emerging, e.g., in mobile and wireless connected units.

An early example of a case where the dynamics itself shapes the topology of a graph is the model of interacting species introduced in Ref. [872]. Here, the authors consider two types (fast and slow) of dynamical variables. Precisely, the fast variables are introduced to model the dynamics of the species' populations, while the slow variables are meant to describe the links of a directed graph, that represents the catalytic interactions among the different species. A relevant result of Ref. [872] is that the graph evolves via mutations of the least fit species. This phenomenon gives rise eventually to an autocatalytic set, that is able to trigger a cascade of exponentially increasing connectivities until spanning the whole graph and yielding a highly non-random network.

Another early example of evolutionary topology is that considered in Ref. [873]. Here the goal was to understand the threshold network structure with asymmetric connections that was encountered in diluted asymmetric spin glasses [874], as well as in diluted asymmetric neural networks [875,876]. To that purpose, the topology of an asymmetrically connected threshold network is evolved via a set of local rewiring rules, through which some nodes gain new links in time, while some others lose part of their edges. The interesting point is that very simple rules are yet able to leads to a critical value for the average network connectivity in the thermodynamic limit, and to generate self-organization in the network. As illustrative examples, Ref. [873] shows how these rules can be applied to neural networks and to regulatory networks of the genome.

Ref. [877] considered an adaptive Boolean network where agents evolve in time their binary state following the so called minority game [878], and operating in a dynamical environment. While the phenomenon of adaptation in similar networks was reported before in Ref. [879], here the authors consider the case of a Kauffman random Boolean network [687]. The different agents adapt their dynamics by choosing among different Boolean functions, and switching from one function to another throughout the game, depending on their actual capacity to predict the winning group. In this context, a noticeable result is that agents making use of local information of the net topology are more efficient in such dynamical environment than agents making use of the global information, thus suggesting that this can explain what occurs in multi-agent systems for the processes of allocation of distributed resources.

Stochastically evolving networks (where new nodes and new links between not yet connected nodes are added at random times) have been proposed in Ref. [880]. Here, both random recursive trees [881], and stochastic models like the ones introduced in Ref. [882] were considered, and the study concerned the derivation of a series of exact results for moments, correlations and degree distributions. In particular, in some of these models, Ref. [880] recovers asymptotic power-law distributions for many properties of the network, thus formulating a dynamical approach for the modelling of scale-free networks.

Recently, an interesting research has been carried out, motivated by the need of properly describing the virus spreading processes in the Internet through e-mail. Many computer viruses, indeed, use a determined mechanism to spread: they first arrive to a recipient (usually as attached files to some e-mail message), and, if activated, they send replicas of themselves to many other users. A possible strategy for the activated virus is to make use of the recipient's e-mail address book and spread itself to the list of users whose e-mail addresses are contained in that address book. In order to reproduce the essential dynamical features of such a mechanism, Ref. [543] investigates a specific model of evolving e-mail networks. Here, the nodes are users' e-mail address books, and the edges are the records of e-mail addresses in such books. Similar approaches with static wirings were introduced also in Refs. [84,539], where, by using real data from server log files or address books of large computer systems, specific models for e-mail networks were designed that displayed scale-free properties. The peculiarity of the approach introduced in Ref. [543] is that, as time goes on, new links are generated and old links removed in the net within specific time intervals. This reflects the process through which the different users reorganize periodically their e-mail address books (e.g. by deleting or adding some e-mail addresses of other users). Furthermore, the balance between generation and deletion processes is taken to depend on 
suitable variable parameters, and therefore it can be modified and controlled in the numerical trials. In particular, Ref. [543] distinguishes between two cases of e-mail contacts: the equivalent e-mail contact (the number of e-mail messages sent by each user is independent on the user's degree), and the degree-related e-mail contact (the number of e-mail messages sent by a single user is related to the size of its address book). In both cases it is found that the essential properties of the network (such as the average number of links, the average path length, or the clustering coefficient) strongly depend on the parameters of the model. In particular, in the case of an equivalent e-mail contact, small-sized evolving networks exhibit a decreasing average path length in time, which (if associated to a high clustering coefficient) recovers the essential properties of a small-world shaped network.

Another recent research [883] modelled evolving cooperation. This phenomenon, commonly observed in many social, economic and biological situations, is represented by means of a set of interacting elements. The interactions follow a spatial game, and the wiring of connections evolves adapting itself to the outcome of the game (e.g., for facilitating the formation of hierarchical structures sustaining collective cooperative states). When a local neighbor selection is taken as the rule for the wiring dynamics, the resulting network exhibits the small-world properties and shows a shaped hierarchical structure of highly connected nodes playing a crucial role for the stability of the cooperation dynamics. In particular, the case is considered of external perturbations acting on the states of such hierarchical structure, and it is shown that global avalanches can be triggered having the result of completely reshaping the entire network structure.

Several self-organization mechanisms in the evolution of social collaboration networks (such as movie actor collaboration, scientific collaboration, etc.) have been studied in Ref. [453]. Here, a model of growing network is dynamically constructed by combining the preferential attachment rule with an imposed bipartite structure. In particular, it is shown that the empirical observations on clustering and degree-degree correlations in real collaboration networks can be accounted for by introducing an aging of collaborators, responsible for the assortative degree-degree correlations.

Wiring evolution processes have been studied also in models of weighted complex networks. An example is the study of Ref. [884], that considered weighted scale-free networks incorporating a fit-gets-richer scheme, and where furthermore the topology of weights in the network evolves as time goes on. Similarly, weighted evolving networks that couple the establishment of new edges and nodes with the weights' dynamical evolution are studied in Ref. [214], showing that a non-trivial evolution of node's properties and scale-free behavior for weight, strength and degree distributions is determined by this kind of models.

Another class of dynamical networks that deserves to be discussed is that modelling the coordination and grouping of animals during their motion, and the swarming processes that are observed ubiquitously in nature. These studies were triggered by a simple, yet elegant, approach introduced by Vicsek et al. [885]. In this approach, a model is introduced of walkers in a two-dimensional plane, with the aim of describing the appearance of a self-ordered motion in the absence of a centralized control, or without a leader walker. The walkers are massless and dimensionless particles, initially distributed randomly in the plane, each one of them having a velocity vector that is fixed in its modulus, but random in its orientation in the plane. Furthermore, each walker is assigned an interaction radius $r$ much smaller than the extension $L$ of the system, and periodic boundary conditions are fixed in both directions of the plane. The evolving rules of the network are rather simple. At each time step, each walker interacts will all the other walkers whose physical distances from it are smaller than $r$. The walker maintains unchanged the modulus of its velocity, and assumes a direction for the velocity vector that is the average direction of its neighboring walkers plus an added noisy term chosen with uniform probability within a given interval $[-\eta / 2, \eta / 2]$. As a result of this procedure, the evolution of the whole system is mapped into an evolving network, where new links are added and old links removed continuously, depending on the relative positions of walkers in the plane. The two relevant parameters of the models are $\eta$ and the density of walkers in the plane $\rho$. When both $\eta$ and $\rho$ are small, the walkers tend to form groups that are coherently moving in random directions. Conversely, for high densities and noises, the walkers move randomly, though showing some form of correlation. Finally, for high densities and small noises, the motion of walkers becomes ordered on the same scale of the system size, and all walkers move in the same spontaneously chosen direction.

The results of the Vicsek et al. model are nowadays stimulating a flurry of researches trying to understand what are the minimal ingredients, e.g., for describing the onset of collective motion (swarming) in moving animals without a leader, or to design schemes for robots coordination in the absence of a centralized control. In particular, a recent study [886] by Grégoire and Chaté, has extended the original Vicsek et al. model by introducing a further cohesive term in the rules for the evolution of the walkers. The relevant result, which promises to shed light onto the mechanisms at the basis of the swarming, is that, in the presence of cohesion, the onset of collective motion is determined by a complex 
interplay among several factors, including the density of walkers, and the shape of the grouped walkers, giving rise to a fascinating dynamics.

Given the practical implications of wiring evolution processes in real networks, the challenging study of adaptive and dynamical topologies is nowadays rapidly growing, and this subject promises to attract much interest of the scientific community in the forthcoming years.

\section{Acknowledgements}

At the end of a such work, we would like to acknowledge all scientists with whom we had (and have) interactions on this topic. Their sharing of very stimulating and fruitful discussions (or unpublished results on the subjects treated in this report) is largely responsible for our effort to provide a new account of this rapidly growing field of research.

In this spirit, the authors would like to thank R. Albert, A. Amann, V. Anishchenko, A. Arenas, A. Barabási, M. Baranger, E. Barreto, B. Behringer, I. Belykh, V. Belykh, E. Ben Jacob, S. Bologna, J. Bragard, G. Caldarelli, T. Carroll, R. Criado, P. Crucitti, P. De Los Rios, A. Diaz-Guilera, M. Ding, P. Echenique, L.M. Floria, L. Fortuna, S. Fortunato, M. Frasca, J. García-Ojalvo, A. Giansanti, B. Gluckman, J. Gómez-Gardeñes, C. Grebogi, S.K. Han, M. Hasler, H. Jeong, B. Kahng, H. Kantz, B.J. Kim, C.-M. Kim, J. Kurths, K. Josić, Y.-C. Lai, M. Le van Quyen, I. Leyva, R. López Ruiz, H. Mancini, R.N. Mantegna, M. Marchiori, M. Marsili, I. Mariño, C. Masoller, D. Maza, M. Menzinger, M. Nekovee, M.E.J. Newman, M. Ogorzalek, E. Ott, A.F. Pacheco, U. Parlitz, R. Pastor-Satorras, Y.-J. Park, L.M. Pecora, L. Pezard, A. Pikovsky, A. Pluchino, A. Politi, S. Porta, I. Procaccia, A. Rapisarda, A. Robledo, V. Rosato, M.G. Rosenblum, M. Rosvall, R. Roy, G. Russo, S. Schiff, I. Sendiña Nadal, K. Showalter, P. So, R.V. Solé, B. Spagnolo, H.E. Stanley, S.H. Strogatz, B. Tadic̀, C. Tsallis, S. Valverde, A. Vázquez, M. Vázquez-Prada, A. Vespignani, T. Vicsek, J. Yorke, M. Zaks, and C. Zhou.

We furthermore would like to thank gratefully the help of the anonymous Referees, whose very tactful and constructive reports have allowed us to greatly improve the present Manuscript.

Work partly supported by the MIUR-FIRB project n. RBNE01CW3M-001, the INFN project FB11, the Spanish DGICYT Project FIS2004-05073-C04-01, and a DGA (Spain) project. Y.M. is supported by MEC through the Ramón y Cajal Program.

\section{Note added in proof}

After the completion of the Report we learned about recent works that are important contributions to the subjects reviewed here. In the following, these additional References are listed, together with the number of the Section they refer to.

\section{Section1.1:}

U. Brandes, T. Erlebach (Eds.), Network Analysis: Methodological Foundations, Springer, Berlin, 2005.

Section 2.1:

K.I. Goh, G. Salvi, B. Kahng, D. Kim, preprint cond-mat/0508332.

Section 2.3.1:

I. Derényi, G. Palla, T. Vicsek, Phys. Rev. Lett. 94 (2005) 160202.

Section 2.4:

G. Bianconi, Europhys. Lett. 71 (2005) 1029.

Section 3.1:

M. Boguñá, M.A. Serrano, Phys. Rev. E 72 (2005) 016106.

Section 5.3:

L. Donetti, P.I. Hurtado, M.A. Muñoz, Phys. Rev. Lett. 95 (2005) 188701.

Section 6.1:

R. Guimerá, B. Uzzi, J. Spiro, L.A.N. Amaral, Science 308 (2005) 697. 
Section 6.3:

R. Albert, J. Cell Sci. 118 (2005) 4947; M.C. Palumbo, A. Colosimo, A. Giuliani, L. Farina, FEBS Letters 579 (2005) 4642; M. Chaves, R. Albert, E.D. Sontag, preprint q-bio.MN/0501037.

Section 7.1:

R. Guimerá, L.A.N. Amaral, Nature 433 (2005) 895; G. Palla, I. Derényi, I. Farkas, T. Vicsek, Nature 435 (2005) 814; J. Duch, A. Arenas, Phys. Rev. E 72 (2005) 027104; L. Danon, A. Diaz-Guilera, J. Duch, A. Arenas, J. Stat. Mech. P09008 (2005).

\section{References}

[1] S.H. Strogatz, Nature 410 (2001) 268.

[2] R. Albert, A.-L. Barabási, Rev. Mod. Phys. 74 (2002) 47.

[3] S.N. Dorogovtesev, J.F.F. Mendes, Adv. Phys. 51 (2002) 1079.

[4] M.E.J. Newman, SIAM Rev. 45 (2003) 167.

[5] D.J. Watts, Small Worlds: The Dynamics of Networks between Order and Randomness, Princeton University Press, Princeton, NJ, 1999.

[6] S. Bornholdt, H.G. Schuster (Eds.), Handbook of Graphs and Networks: From the Genome to the Internet, Wiley-VCH, Germany, 2003.

[7] S.N. Dorogovtesev, J.F.F. Mendes, Evolution of Networks, Oxford University Press, Oxford, 2003.

[8] R. Pastor-Satorras, A. Vespignani, Evolution and Structure of the Internet: A Statistical Physics Approach, Cambridge University Press, Cambridge, 2004.

[9] R. Pastor-Satorras, M. Rubi, A. Diaz-Guilera (Eds.), Statistical Mechanics of Complex Networks, Springer, Berlin, 2003.

[10] E. Ben-Naim, H. Frauenfelder, Z. Toroczkai (Eds.), Complex Networks, Springer, Berlin, 2004.

[11] M. Buchanan, Nexus: Small Worlds and the Groundbreaking Science of Networks, Norton, New York, 2002.

[12] A.-L. Barabási, Linked: The New Science of Networks, Perseus, Cambridge, 2002.

[13] D.J. Watts, Six Degrees: The Science of a Connected Age, Norton, New York, 2003.

[14] B. Bollobás, Random Graphs, Academic Press, London, 1985.

[15] B. Bollobás, Modern Graph Theory, Graduate Texts in Mathematics, Springer, New York, 1998.

[16] D.B. West, Introduction to Graph Theory, Prentice-Hall, Englewood Cliffs, NJ, 1995.

[17] F. Harary, Graph Theory, Perseus, Cambridge, MA, 1995.

[18] S. Wasserman, K. Faust, Social Networks Analysis, Cambridge University Press, Cambridge, 1994.

[19] J. Scott, Social Network Analysis: A Handbook, 2nd ed., Sage Publications, London, 2000.

[20] T.H. Cormen, C.E. Leiserson, R.L. Rivest, C. Stein, Introduction to Algorithms, MIT University Press, Cambridge, 2001.

[21] R. Sedgewick, Algorithms in C + +. Part 5: Graph Algorithms, Addison-Wesley, Boston MA, 1988.

[22] R.K. Ahuja, T.L. Magnati, J.B. Orlin, Network Flows: Theory, Algorithms, and Applications, Prentice-Hall, Englewood Cliffs, NJ, 1993.

[23] M. Boguñá, R. Pastor-Satorras, Phys. Rev. E 66 (2002) 047104.

[24] M. Boguñá, R. Pastor-Satorras, A. Vespignani, Lect. Notes Phys. 625 (2003) 127.

[25] R. Pastor-Satorras, A. Vázquez, A. Vespignani, Phys. Rev. Lett. 87 (2001) 258701.

[26] M.E.J. Newman, Phys. Rev. Lett. 89 (2002) 208701.

[27] M.E.J. Newman, Phys. Rev. E 67 (2003) 026126.

[28] D.J. Watts, S.H. Strogatz, Nature 393 (1998) 440.

[29] M. Marchiori, V. Latora, Physica A 285 (2000) 539.

[30] V. Latora, M. Marchiori, Phys. Rev. Lett. 87 (2001) 198701.

[31] V. Latora, M. Marchiori, Eur. Phys. J. B 32 (2003) 249.

[32] R. Criado, A. García del Amo, B. Hernández-Bermejo, M. Romance, J. Comput. Appl. Math., 2005, in press.

[33] J. Buhl, J. Gautrais, R.V. Solé, P. Kuntz, S. Valverde, J.L. Deneubourg, G. Theraulaz, Eur. Phys. J. B 42 (2004) 123.

[34] V. Latora, M. Marchiori, Phys. Rev. E 71 (2005) 015103(R).

[35] R. Criado, J. Flores, B. Hernández-Bermejo, J. Pello, M. Romance, J. Math. Model. Algorithms 4 (2005) 307.

[36] I. Vragovi`c, E. Louis, A. Di`az-Guilera, Phys. Rev. E 71 (2005) 036122.

[37] V. Latora, M. Marchiori, preprint cond-mat/0402050.

[38] C.L. Freeman, Sociometry 40 (1977) 35.

[39] L.C. Freeman, Social Networks 1 (1979) 215.

[40] M.E.J. Newman, Phys. Rev. E 64 (2001) 016132.

[41] U. Brandes, J. Math. Soc. 25 (2001) 163.

[42] K.I. Goh, B. Kahng, D. Kim, Phys. Rev. Lett. 87 (2001) 278701.

[43] K.I. Goh, E.S. Oh, H. Jeong, B. Kahng, D. Kim, Proc. Natl. Acad. Sci. USA 99 (2002) 125832.

[44] M. Barthélemy, Phys. Rev. Lett. 91 (2003) 189803.

[45] K.I. Goh, C.M. Ghim, B. Kahng, D. Kim, Phys. Rev. Lett. 91 (2003) 189804.

[46] M. Barthélemy, Eur. Phys. J. B 38 (2004) 163.

[47] P. Crucitti, V. Latora, S. Porta, preprint physics/0504163. 
[48] K.-I. Goh, E. Oh, B. Kahng, D. Kim, Phys. Rev. E 67 (2003) 017101.

[49] R. Guimerà, S. Mossa, A. Turtschi, L.A.N. Amaral, Proc. Natl. Acad. Sci. USA 102 (2005) 7794;

R. Guimerà, L.A.N. Amaral, Eur. Phys. J. B 38 (2004) 381.

[50] P. Crucitti, V. Latora, M. Marchiori, Physica A 338 (2004) 92.

[51] M.E.J. Newman, M. Girvan, Phys. Rev. E 69 (2004) 026113.

[52] M.E.J. Newman, Phys. Rev. E 64 (2001) 016131.

[53] A. Barrat, M. Weigt, Eur. Phys. J. B 13 (2000) 547.

[54] N. Alon, R. Yuster, U. Zwick, Algorithmica 17 (1997) 209.

[55] B. Jiang, C. Claramunt, Environ. Plann. B 31 (2004) 151.

[56] A. Fronczak, J.A. Holyst, M. Jedynak, J. Sienkiewicz, Physica A 316 (2002) 688.

[57] G. Caldarelli, R. Pastor-Satorras, A. Vespignani, Eur. Phys. J. B 38 (2004) 183.

[58] G. Bianconi, A. Capocci, Phys. Rev. Lett. 90 (2003) 078701.

[59] M.E.J. Newman, Social Networks 25 (2003) 83.

[60] S.N. Soffer, A. Vázquez, Phys. Rev. E 71 (2005) 057101.

[61] S. Wasserman, J. Math. Sociol. 5 (1977) 61.

[62] S. Shen-Orr, R. Milo, S. Mangan, U. Alon, Nature Gen. 31 (2002) 64.

[63] R. Milo, S. Shen-Orr, S. Itzkovitz, N. Kashan, D. Chklovskii, U. Alon, Science 298 (2002) 824.

[64] S. Mangan, U. Alon, Proc. Natl. Acad. Sci. USA 100 (2003) 11980.

[65] R. Milo, S. Itzkovitz, N. Kashtan, R. Levitt, S. Shen-Orr, I. Ayzenshtat, M. Sheffer, U. Alon, Science 303 (2004) 1538.

[66] N. Kashtan, S. Itzkovitz, R. Milo, U. Alon, Bioinformatics 20 (2004) 1746.

[67] R.D. Alba, J. Math. Sociol. 3 (1973) 113.

[68] G.W. Flake, S.R. Lawrence, C.L. Giles, F.M. Coetzee, IEEE Comput. 35 (2002) 66.

[69] S.B. Seidman, Social Networks 5 (1983) 97.

[70] S.P. Borgatti, M.G. Everett, P.R. Shirey. Social Networks 12 (1990) 337.

[71] F. Radicchi, C. Castellano, F. Cecconi, V. Loreto, D. Parisi, Proc. Natl. Acad. Sci. USA 101 (2004) 2658.

[72] I. Farkas, I. Derényi, A.-L. Barabási, T. Vicsek, Phys. Rev E 64 (2001) 26704.

[73] K.-I. Goh, B. Kahng, D. Kim, Phys. Rev. E 64 (2001) 051903.

[74] A.J. Seary, W.D. Richards, in: Dynamic Social Network Modeling and Analysis, National Academy Press, Washington, DC, pp. $209-228$.

[75] N. Biggs, Algebraic Graph Theory, Cambridge University Press, Cambridge, 1993.

[76] D. Vukadinovic, P. Huang, T. Erlebach T. ETH Zurich, TIK Report No. 118, July 2001.

[77] V. Rosato, F. Tiriticco, Europhys. Lett. 66 (2004) 471.

[78] M. Mehta, Random Matrices, Academic Press, New York, 1995.

[79] Y. Bar-Yam, Dynamics of Complex Systems, Addison-Wesley, Reading, MA, 1997.

[80] A. Vázquez, R. Pastor-Satorras, A. Vespignani, Lect. Notes Phys. 650 (2004) 425.

[81] R. de Castro, J.W. Grossman, Math. Intell. 21 (1999) 51.

[82] J.W. Grossman, P.D.F. Ion, Congr. Numer. 108 (1995) 129.

[83] L.A.N. Amaral, A. Scala, M. Barthélemy, H.E. Stanley, Proc. Natl. Acad. Sci. (USA) 97 (2000) 11149.

[84] H. Ebel, L.-I. Mielsch, S. Bornholdt, Phys. Rev. E 66 (2002) 035103.

[85] H. Jeong, B. Tombor, R. Albert, Z.N. Oltvai, A.-L. Barabási, Nature 407 (2000) 651.

[86] H. Jeong, S.P. Mason, A.-L. Barabási, Z.N. Oltvai, Nature 411 (2001) 41.

[87] R.V. Solé, J.M. Montoya, Proc. R. Soc. London B 268 (2001) 2039.

[88] J. Camacho, R. Guimerá, L.A.N. Amaral, Phys. Rev. Lett. 88 (2002) 228102.

[89] S. Milgram, Psychol. Today 1 (1967) 60.

[90] J. Travers, S. Milgram, Sociometry 32 (1969) 425.

[91] C. Korte, S. Milgram, J. Personal. Social Psychol. 15 (1970) 101.

[92] P.S. Dodds, R. Muhamad, D.J. Watts, Science 301 (2003) 827.

[93] A.-L. Barabási, R. Albert, Science 286 (1999) 509.

[94] H.E. Stanley, Introduction to Phase Transitions and Critical Phenomena, Oxford University Press, New York, 1971.

[95] K.J. Falconer, Fractal Geometry: Mathematical Foundations and Applications, Wiley, 1990.

[96] C. Song, S. Havlin, H.A. Makse, Nature 433 (2005) 392.

[97] The national laboratory for applied network research (NLANR), National Science Foundation, http://moat.nlanr.net/

[98] Mapping the internet within the scan project, Information Sciences Institute, http://www.isi.edu/div7/scan/

[99] A. Oram (Ed.), Peer-to-Peer: Harnessing the Power of Disruptive Technologies, O’Reilly \& Associates, Inc., Sebastopol, CA, 2001.

[100] A. Vázquez, Phys. Rev. E 67 (2003) 056104.

[101] A. Broder, R. Kumar, F. Maghoul, P. Raghavan, S. Rajagopalan, R. Stata, A. Tomkins, J. Wiener, Computer Networks 33 (2000) 309.

[102] P. Echenique, J. Gómez-Gardeñes, Y. Moreno, A. Vázquez, Phys. Rev. E 71 (2005) 035102(R).

[103] E. Ravasz, A.L. Somera, D.A. Mongru, Z.N. Oltvai, A.-L. Barabási, Science 297 (2002) 1551.

[104] J.-C. Rain, et al., Nature 409 (2001) 211.

[105] U. Alon, M. Surette, N. Barkai, S. Leiber, Nature 397 (1999) 168.

[106] A. Eldar, et al., Nature 419 (2002) 304.

[107] J.M. Stuart, et al., Science 302 (2003) 249.

[108] S. Wuchty, Z.N. Oltvai, A.-L. Barabási, Nat. Genet. 35 (2003) 176. 
[109] A. Vázquez, et al., Nat. Biotech. 21 (2003) 697.

[110] J.M. Montoya, R.V. Solé, J. Theoret. Biol. 214 (2002) 405.

[111] R.J. Williams, E.L. Berlow, J.A. Dunne, A.-L. Barabási, Proc. Natl. Acad. Sci. USA 99 (2002) 12913.

[112] J.A. Dunne, R.J. Williams, N.D. Martinez, Proc. Natl. Acad. Sci. USA 99 (2002) 12917.

[113] D. Garlaschelli, G. Caldarelli, L. Pietronero, Nature 423 (2003) 165.

[114] A. Rapoport, Bull. Math. Biophys. 19 (1957) 257.

[115] P. Erdös, A. Rényi, Publ. Math. Debrecen 6 (1959) 290.

[116] Z. Burda, J. Jurkiewicz, A. Krzywicki, Physica A 344 (2004) 56.

[117] P. Erdös, A. Rényi, Publ. Math. Inst. Hung. Acad. Sci. 5 (1960) 17.

[118] J. Park, M.E.J. Newman, Phys. Rev. E 70 (2004) 066117.

[119] B. Bollobás, Discrete Math. 33 (1981) 1.

[120] F. Chung, L. Lu, Adv. Appl. Math. 26 (2001) 257.

[121] E.A. Bender, E.R. Canfield, J. Combin. Theory A 24 (1978) 296.

[122] M. Molloy, B. Reed, Random Struct. Algorithm 6 (1995) 161.

[123] M. Molloy, B. Reed, Combin. Probab. Comput. 7 (1998) 295.

[124] M.E.J. Newman, S.H. Strogatz, D.J. Watts, Phys. Rev. E 64 (2001) 26118.

[125] F. Chung, L. Lu, Proc. Natl. Acad. Sci. USA 99 (2002) 15879.

[126] J. Davidsen, H. Ebel, S. Bornholdt, Phys. Rev. Lett. 88 (2002) 128701.

[127] R. Monasson, Eur. Phys. J. B 12 (1999) 555.

[128] M.E.J. Newman, D.J. Watts, Phys. Lett. A 263 (1999) 341.

[129] M. Barthélemy, L.A.N. Amaral, Phys. Rev. Lett. 82 (1999) 3180.

[130] M. Argollo de Menezes, C.F. Moukarzel, T.J.P. Penna, Europhys. Lett. 50 (2000) 574.

[131] M.E.J. Newman, C. Moore, D.J. Watts, Phys. Rev. Lett. 84 (2000) 3201.

[132] W. Aiello, F. Chung, L. Lu, Proceedings of the 32nd Annual ACM Symposium on Theory of Computing, Association of Computing Machinery, New York, 2000, p. 171.

[133] R. Cohen, S. Havlin, Phys. Rev. Lett. 90 (2003) 58701.

[134] F. Chung, L. Lu, V. Vu, Proc. Natl. Acad. Sci. USA 100 (2003) 6313.

[135] G. Caldarelli, A. Capocci, P. De Los Rios, M.A. Muñoz, Phys. Rev. Lett. 89 (2002) 258702.

[136] N. Masuda, H. Miwa, N. Konno, Phys. Rev. E 70 (2004) 36124.

[137] Z. Toroczkai, K.E. Bassler, Nature 428 (2004) 716.

[138] Z. Toroczkai, B. Kozma, K.E. Bassler, N.W. Hengartner, G. Korniss, preprint cond-mat/0408262.

[139] L.da F. Costa, preprint cond-mat/0410230.

[140] D.J.de S. Price, J. Amer. Soc. Inform. Sci. 27 (1976) 292.

[141] D.J.de S. Price, Science 149 (1965) 510.

[142] H.A. Simon, Biometrika 42 (1955) 425.

[143] S. Bornholdt, H. Ebel, Phys. Rev. E 64 (2001) 035104.

[144] A.-L. Barabási, R. Albert, H. Jeong, Physica A 272 (1999) 173.

[145] P.L. Krapivsky, S. Redner, F. Leyvraz, Phys. Rev. Lett. 85 (2000) 4629.

[146] S.N. Dorogovtsev, J.F.F. Mendes, A.N. Samukhin, Phys. Rev. Lett. 85 (2000) 4633.

[147] P.L. Krapivsky, S. Redner, Phys. Rev. E 63 (2001) 66123.

[148] R. Xulvi-Brunet, I.M. Sokolov, Phys. Rev. E 70 (2004) 066102.

[149] B. Bollobás, O. Riordan, Preprint, Department of Mathematical Sciences, University of Memphis, 2002.

[150] S.N. Dorogovtsev, J.F.F. Mendes, Phys. Rev. E 63 (2001) 025101.

[151] B.A. Huberman, L.A. Adamic, Nature 401 (1999) 131.

[152] K.-I. Goh, B. Kahng, D. Kim, Phys. Rev. Lett. 88 (2002) 108701.

[153] J. Gómez-Gardeñes, Y. Moreno, Phys. Rev. E 69 (2004) 37103.

[154] R. Albert, A.-L. Barabási, Phys. Rev. Lett. 85 (2000) 5234.

[155] S.N. Dorogovtsev, J.F.F. Mendes, Europhys. Lett. 52 (2000) 33.

[156] B. Tadić, Physica A 293 (2001) 273.

[157] B. Tadić, Physica A 314 (2002) 278.

[158] P.L. Krapivsky, G.J. Rodgers, S. Redner, Phys. Rev. Lett. 86 (2001) 5401.

[159] D.S. Callaway, J.E. Hopcroft, J.M. Kleinberg, M.E.J. Newman, S.H. Strogatz, Phys. Rev. E 64 (2001) 041902.

[160] S.N. Dorogovtsev, J.F.F. Mendes, A.N. Samukhin, Phys. Rev. E 64 (2001) 066110.

[161] P.I. Krapivsky, S. Redner, Comput. Networks 39 (2002) 261.

[162] G.J. Rodgers, K. Darby-Dowman, Eur. Phys. J. B 23 (2001) 267.

[163] G. Bianconi, A.-L. Barabási, Europhys. Lett. 54 (2001) 436.

[164] G. Bianconi, A.-L. Barabási, Phys. Rev. Lett. 86 (2001) 5632.

[165] E. Ravasz, A.-L. Barabási, Phys. Rev. E 67 (2003) 026112.

[166] A.-L. Barabási, E. Ravasz, T. Vicsek, Physica A 299 (2001) 559.

[167] S.N. Dorogovtsev, A.V. Goltsev, J.F.F. Mendes, Phys. Rev. E 65 (2002) 066122.

[168] S. Jung, S. Kim, B. Kahng, Phys. Rev. E 65 (2002) 056101.

[169] P. Holme, B.J. Kim, Phys. Rev. E 65 (2002) 26107. 
[170] G. Szabó, M. Alava, J. Kertész, Phys. Rev. E 67 (2003) 056102.

[171] E.M. Jin, M. Girvan, M.E.J. Newman, Phys. Rev. E 64 (2001) 46132.

[172] K. Klemm, V.M. Eguíluz, Phys. Rev. E 65 (2002) 36123.

[173] A. Vázquez, M. Boguñá, Y. Moreno, R. Pastor-Satorras, A. Vespignani, Phys. Rev. E 67 (2003) 46111.

[174] K. Klemm, V.M. Eguíluz, Phys. Rev. E 65 (2002) 57102.

[175] R.V. Solé, R. Pastor-Satorras, E. Smith, T.B. Kepler, Adv. Complex Systems 5 (2002) 43.

[176] J. Kim, P.L. Krapivsky, B. Kahng, S. Redner, Phys. Rev. E 66 (2002) 55101.

[177] A. Vázquez, A. Flammini, A. Maritan, A. Vespignani, Complexus 1 (2003) 38.

[178] J. Berg, M. Lässig, A. Wagner, BMC Evol. Biol. 4 (2004) 51.

[179] G.P. Karev, Y.I. Wolf, A.Y. Rzhetsky, F.S. Berezovskaya, E.V. Koonin, BMC Evol. Biol. 2 (2002) 18.

[180] J. Kor, Phys. Soc. 46 (2005) 565.

[181] R. Ferrer, I. Cancho, R.V. Solé, Lect. Notes Phys. 625 (2003) 114.

[182] S. Valverde, R. Ferrer i Cancho, R.V. Solé, Europhys. Lett. 60 (2002) 512.

[183] M. Granovetter, American J. Sociol. 78 (1973) 1360.

[184] M.E.J. Newman, Proc. Natl. Acad. Sci. USA 98 (2001) 404.

[185] A.-L. Barabási, H. Jeong, R. Ravasz, Z. Neda, T. Vicsek, A. Schubert, Physica A 311 (2002) 590.

[186] P. Csermely, Trends Biochem. Sci. 29 (2004) 331.

[187] G.A. Polis, Nature 395 (1998) 744.

[188] K. McCann, A. Hastings, G.R. Huxel, Nature 395 (1998) 794.

[189] E.L. Berlow, Nature 398 (1999) 330.

[190] A.E. Krause, K.A. Frank, D.M. Mason, R.E. Ulanowicz, W.W. Taylor, Nature 426 (2003) 282.

[191] O. Sporns, G. Tononi, G.M. Edelman, Neural Networks 13 (2002) 909.

[192] O. Sporns, Complexity 8 (2003) 56.

[193] W. Li, X. Cai, Phys. Rev. E 69 (2004) 046106.

[194] A. Barrat, M. Barthélemy, R. Pastor-Satorras, A. Vespignani, Proc. Natl. Acad. Sci. USA 101 (2004) 3747.

[195] Bo Hu, Xin-Yu Jiang, Jun-Feng Ding, Yan-Bo Xie, B.-H. Wang, preprint cond-mat/0408125.

[196] M. Barthélemy, A. Barrat, R. Pastor-Satorras, A. Vespignani, Physica A 346 (2005) 34.

[197] S.H. Yook, H. Jeong, A.-L. Barabási, Y. Tu, Phys. Rev. Lett. 86 (2001) 5835.

[198] J.P. Onnela, A. Chakraborti, K. Kaski, J. Kertész, A. Kanto, Phys. Rev. E 68 (2003) 056110.

[199] E. Almaas, B. Kovacs, T. Viscek, Z.N. Oltvai, A.-L. Barabási, Nature 427 (2004) 839.

[200] B. Derrida, H. Flyvbjerg, J. Phys. A 20 (1987) 5273.

[201] M. Barthélemy, B. Gondran, E. Guichard, Physica A 319 (2003) 633.

[202] J. Noh, H. Rieger, Phys. Rev. E 66 (2002) 066127.

[203] J.P. Onnela, J. Jari Saramäki, J. Kertész, K. Kaski, Phys. Rev. E 71 (2005) 065103.

[204] S.A. Wagner, D.A. Fell, Proc. R. Soc. London B 268 (2001) 1803.

[205] S. Maslov, K. Sneppen, Science 296 (2002) 910.

[206] P.J. Macdonald, E. Almaas, A.-L. Barabási, Europhys. Lett. 72 (2005) 308.

[207] P. Tieri, S. Valensin, V. Latora, G.C. Castellani, M. Marchiori, D. Remondini, C. Franceschi, Bioinformatics 21 (2005) 1639.

[208] http://xxx.lanl.gov/archive/cond-mat.

[209] S. Zhou, R.J. Mondragon, e-print cs.NI0303028 (2003).

[210] S.N. Dorogovtsev, J.F.F. Mendes, preprint cond-mat/0408343.

[211] E. Almaas, P.L. Krapivsky, S. Redner, Phys. Rev. E 71 (2005) 036124.

[212] D. Zheng, S. Trimper, B. Zheng, P.M. Hui, Phys. Rev. E 67 (2003) 040102.

[213] T. Antal, P.L. Krapivsky, Phys. Rev. E 71 (2005) 026103.

[214] A. Barrat, M. Barthélemy, A. Vespignani, Phys. Rev. Lett. 92 (2004) 228701.

[215] A. Barrat, M. Barthélemy, A. Vespignani, Phys. Rev. E 70 (2004) 066149.

[216] A. Barrat, M. Barthélemy, A. Vespignani, Lect. Notes Comput. Sci. 56 (2004) 3243.

[217] R.V.R. Pandya, preprint cond-mat/0406644 (2004).

[218] S.N. Dorogovtsev, J.F.F. Mendes, A.N. Samukhin, Phys.Rev. E 63 (2001) 062101.

[219] C. Li, G. Chen, Physica A 33 (2004) 288.

[220] C. Fu, et al., preprint cond-mat/0408155.

[221] M. Baiesi, M. Paczuski, Int. J. Mod. Phys. B 18 (2004) 3095.

[222] D. Garlaschelli, S. Battiston, M. Castri, V.D.P. Servedio, G. Caldarelli, Physica A 350 (2005) 491.

[223] G. Yan, T. Zhou, J. Wang, Z. Fu, B. Wang, Chinese Phys. Lett. 22 (2005) 510.

[224] L. Hufnagel, D. Brockmann, T. Geisel, Proc. Natl. Acad. Sci. USA 101 (2004) 15124.

[225] R. Albert, I. Albert, G.L. Nakarado, Phys. Rev. E 69 (2004) 025103(R).

[226] F. Pitts, The Profess. Geograph. 17 (1965) 15.

[227] D.A. Smith, M. Timberlake, Urban Stud. 32 (1995) 287.

[228] V. Latora, M. Marchiori, Physica A 314 (2002) 109.

[229] M. Batty, P. Longley, Fractal Cities, Academic Press, New York, 1994.

[230] S.-H. Yook, H. Jeong, A.-L. Barabási, Proc. Natl. Acad. Sci. USA 99 (2002) 13382.

[231] Govindan, H. Tangmunarunkit, Proc. IEEE INFOCOM '00, Tel Aviv, March 26, 2000, pp. 1371. 
[232] A.K.M. Stoneham, Environ. Plann. A 9 (1977) 185.

[233] G. Csanyi, B. Szendroi, Phys. Rev. E 70 (2004) 016122.

[234] S.P. Gorman, R. Kulkarni, cond-mat/0310426.

[235] J.M. Kleinberg, Nature 406 (2000) 845.

[236] J.M. Kleinberg, Proceedings of the 32nd Annual ACM Symposium on Theory of Computing, Association of Computing Machinery, New York, 2000, p. 163.

[237] S. Jespersen, A. Blumen, Phys. Rev. E 62 (2000) 6270.

[238] P. Sen, B.K. Chakrabarti, J. Phys. A 34 (2001) 7749.

[239] C.F. Moukarzel, M. Argollo de Menezes, Phys. Rev. E 65 (2002) 056709.

[240] P. Sen, K. Banerjee, T. Biswas, Phys. Rev. E 66 (2002) 037102.

[241] T. Petermannn, P. De Los Rios, cond-mat/0501420.

[242] R. Xulvi-Brunet, I.M. Sokolov, Phys. Rev. E 66 (2002) 026118.

[243] S.S. Manna, P. Sen, Phys. Rev. E 66 (2002) 066114.

[244] D.J.B. Soares, C. Tsallis, A.M. Mariz, L.R. da Silva, Europhys. Lett. 70 (2005) 70.

[245] M. Barthélemy, Europhys. Lett. 63 (2003) 915.

[246] J. Jost, M.P. Joy, Phys. Rev. E 66 (2002) 036126.

[247] M. Kaiser, C.C. Higeltag, Phys. Rev. E 69 (2004) 036103.

[248] A.F. Rozenfeld, R. Cohen, D. ben-Avraham, S. Havlin, Phys. Rev. Lett. 89 (2002) 218701.

[249] C.P. Warren, L.M. Sander, I.M. Sokolov, Phys. Rev. E 66 (2002) 056105.

[250] K. Yang, L. Huang, L. Yang, Phys. Rev. E 70 (2004) 015102(R).

[251] R. Pastor-Satorras, A. Vespignani, Phys. Rev. Lett. 86 (2001) 3200.

[252] R. Pastor-Satorras, A. Vespignani. Phys. Rev. E 63 (2001) 066117.

[253] C. Herrmann, M. Barthélemy, P. Provero, Phys. Rev. E 68 (2003) 026128.

[254] J. Dall, M. Christensen, Phys. Rev. E 66 (2002) 016121.

[255] G. Nemeth, G. Vattay, Phys. Rev. E 67 (2003) 036110.

[256] I.J. Farkas, H. Jeong, T. Vicsek, A.-L. Barabási, Z.N. Oltvai, Physica A 318 (2003) 601.

[257] P. Provero, e-print cond-mat/0207345.

[258] K. Rho, H. Jeong, B. Kahng, Physica A, in press.

[259] J. Rougemont, P. Hingamp, BMC Bioinformatics 4 (2003) 15.

[260] H. Agrawal, Phys. Rev. Lett. 89 (2002) 268702.

[261] S. Mossa, M. Barthélemy, H.E. Stanley, L.A.N. Amaral, Phys. Rev. Lett. 88 (2002) 138701.

[262] B. Waxman, Routing of multipoint connections, IEEE J. Selec. Areas Commun. 6 (1988) 1617.

[263] J.M. Carlson, J. Doyle, Phys. Rev. E 60 (1999) 1412.

[264] A. Fabrikant, E. Koutsoupias, C.H. Papadimitriou, in: P. Widmayer, et al. (Eds.), Heuristically Optimized Trade-Offs: A New Paradigm for Power Laws in the Internet ICALP 2002, Lecture Notes in Computer Science, vol. 2380, Springer, Berlin, Heidelberg, 2002 , p. 110122.

[265] M.T. Gastner, M.E.J. Newman, cond-mat/0407680.

[266] M.E.J. Newman, D.J. Watts, Phys. Rev. E 60 (1999) 7332.

[267] S. Kirkpatrick, C.D. Gelatt, M.P. Vecchi, Science 220 (1983) 671.

[268] M.T. Gastner, M.E.J. Newman, cond-mat/0409702.

[269] S. Porta, P. Crucitti, V. Latora, preprint physics/0506009.

[270] B. Hillier, J. Hanson, The Social Logic of Space, Cambridge University Press, Cambridge, UK, 1984.

[271] B. Hillier, Space is the Machine: A Configurational Theory of Architecture, Cambridge University Press, Cambridge, UK, 1996.

[272] M. Rosvall, A. Trusina, P. Minnhagen, K. Sneppen, Phys. Rev. Lett. 94 (2005) 028701.

[273] M. Batty, Environ. Plann. B 28 (2001) 637.

[274] S. Porta, P. Crucitti, V. Latora, preprint cond-mat/0411241.

[275] R. Albert, H. Jeong, A.-L. Barabási, Nature 406 (2000) 378.

[276] D.S. Callaway, M.E.J. Newman, S.H. Strogatz, D.J. Watts, Phys. Rev. Lett. 85 (2000) 5468.

[277] R. Cohen, K. Erez, D. ben Avraham, S. Havlin, Phys. Rev. Lett. 85 (2000) 4626.

[278] R. Cohen, K. Erez, D. ben Avraham, S. Havlin, Phys. Rev. Lett. 86 (2001) 3682.

[279] R. Cohen, D. ben Avraham, S. Havlin, Phys. Rev. E 66 (2002) 36113.

[280] A. Vázquez, Y. Moreno, Phys. Rev. E 67 (2003) 015101(R).

[281] D. Stauffer, A. Aharony, Introduction to Percolation Theory, Taylor \& Francis, London, 1991.

[282] P. Crucitti, V. Latora, M. Marchiori, A. Rapisarda, Physica A 320 (2003) 622.

[283] P. Crucitti, V. Latora, M. Marchiori, A. Rapisarda, Physica A 340 (2004) 388.

[284] J.-H. Kim, K.-I. Goh, B. Kahng, D. Kim, Phys. Rev. Lett. 91 (2003) 058701.

[285] A.E. Motter, T. Nishikawa, Y. Lai, Phys. Rev. E 66 (2002) 065103.

[286] P. Holme, B.J. Kim, Phys. Rev. E 65 (2002) 066109.

[287] P. Holme, Phys. Rev. E 66 (2002) 036119.

[288] A. Vázquez, M. Weigt, Phys. Rev. E 67 (2003) 027101(R).

[289] M. Leone, A. Vázquez, A. Vespignani, R. Zecchina, Eur. Phys. J. B 28 (2002) 191.

[290] N. Sator, Phys. Rep. 376 (2003) 1.

[291] B.A. Carreras, D.E. Newman, I. Dolrou, A.B. Poole, in: Proceedings of Hawaii International Conference on System Sciences, January 4-7, 2000, Maui, Hawaii. 
[292] M.L. Sachtjen, B.A. Carreras, V.E. Lynch, Phys. Rev. E 61 (2000) 4877.

[293] J. Glanz, R. Perez-Pena, 90 Seconds That Left Tens of Millions of People in the Dark, New York Times, August 26, 2003.

[294] Y. Moreno, J.B. Gomez, A.F. Pacheco, Europhys. Lett. 58 (2002) 630.

[295] H.J. Herrman, S. Roux (Eds.), Statistical Models for the Fracture of Disordered Media, North-Holland, Amsterdam, 1990.

[296] Y. Moreno, J.B. Gómez, A.F. Pacheco, Phys. Rev. Lett. 85 (2000) 2865.

[297] Y. Moreno, R. Pastor-Satorras, A. Vázquez, A. Vespignani, Europhys. Lett. 62 (2003) 292.

[298] A.E. Motter, Y. Lai, Phys. Rev. E 66 (2002) 065102(R).

[299] A.E. Motter, Phys. Rev. Lett. 93 (2004) 098701.

[300] P. Crucitti, V. Latora, M. Marchiori, Phys. Rev. E 69 (2004) 045104(R).

[301] R. Kinney, P. Crucitti, R. Albert, V. Latora, Eur. Phys. J. B 46 (2005) 101.

[302] T.J. Overbay, Am. Scientist 88 (2000) 220.

[303] Electricity Technology Roadmap, 1999 Summary and Synthesis, by the Electric Power Research Institute, http://www.epri.com/ corporate/discover_epri/roadmap/

[304] A.-L. Barabási, The New York Times, August 16, 2003.

[305] S.H. Strogatz, The New York Times, August 25, 2003.

[306] B.A. Carreras, V.E. Lynch, I. Dobson, D.E. Newman, Chaos 12 (2002) 985.

[307] A.J. Wood, B.F. Wollenberg, Power Generation, Operation and Control, Wiley, New York, 1984.

[308] X.F. Wang, J. Xu, Phys. Rev. E 70 (2004) 056113.

[309] K. Kaneko, Coupled Map Lattices, World Scientific, Singapore, 1992.

[310] R.N. Mantegna, H.E. Stanley, An Introduction to Econophysics: Correlations and Complexity in Finance, Cambridge University Press, Cambridge, 2000.

[311] D.J. Watts, Proc. Natl. Acad. Sci. USA 99 (2002) 5766.

[312] Y. Moreno, A. Vázquez, Europhys. Lett. 57 (2002) 765.

[313] P. Bak, K. Sneppen, Phys. Rev. Lett. 71 (1993) 4083.

[314] H. Jensen, Self-Organized Criticality, Cambridge University Press, New York, 1998.

[315] L. de Arcangelis, H.J. Herrmann, Physica A 308 (2002) 545.

[316] K.I. Goh, D.S. Lee, B. Kahng, D. Kim, Phys. Rev. Lett. 91 (2003) 148701.

[317] F. Caruso, V. Latora, A. Rapisarda, B. Tadić, preprint cond-mat/0507643.

[318] T.C. Schelling, J. Conflict Resol. 17 (1973) 381.

[319] V. Jacobson, Comput. Comm. Rev. 18 (1988) 314.

[320] R. Guimerà, A. Arenas, A. Díaz-Guilera, F. Giralt, Phys. Rev. E 66 (2002) 026704.

[321] T. Ohira, R. Sawatari, Phys. Rev. E 58 (1998) 193.

[322] R.V. Solé, S. Valverde, Physica A 289 (2001) 595.

[323] S. Valverde, R.V. Solé, Physica A 312 (2002) 636.

[324] M. Argollo de Menezes, A.-L. Barabási, Phys. Rev. Lett. 92 (2004) 028701.

[325] S. Valverde, R.V. Solé, Eur. Phys. J. B 38 (2004) 245.

[326] B. Tadić, G.J. Rodgers, Adv. Complex Systems 5 (2002) 445.

[327] B. Tadić, S. Thurner, Physica A 332 (2004) 566.

[328] B. Tadić, S. Thurner, G.J. Rodgers, Phys. Rev. E 69 (2004) 036102.

[329] M. Takayasu, H. Takayasu, T. Sato, Physica A 233 (1996) 824.

[330] B. Tadić, S. Thurner, Physica A 346 (2005) 183.

[331] A. Arenas, A. Di`az-Guilera, R. Guimerá, Phys. Rev. Lett. 86 (2001) 3196.

[332] R. Guimerà, A. Arenas, A. Díaz-Guilera, Physica A 299 (2001) 247.

[333] R. Guimerà, A. Díaz-Guilera, F. Vega-Redondo, A. Cabrales, A. Arenas, Phys. Rev. Lett. 89 (2002) 248701.

[334] P. Holme, Adv. Complex Systems 6 (2003) 163.

[335] P. Echenique, J. Gómez-Gardeñes, Y. Moreno, Phys. Rev. E 70 (2004) 056105.

[336] P. Echenique, J. Gómez-Gardeñes, Y. Moreno, Europhys. Lett. 71 (2005) 325.

[337] M. Woolf, et al., Phys. Rev. E 66 (2002) 046106.

[338] B.K. Singh, N. Gupte, Phys. Rev. E 68 (2003) 066121.

[339] B.K. Singh, N. Gupte, Phys. Rev. E 71 (2005) 055103(R).

[340] A.T. Lawniczak, K.P. Maxie, A. Gerisch, Lect. Notes Comput. Sci. 3305 (2004) 325.

[341] J. von Neumann, Theory of Self-Reproducing Automata, University of Illinois Press, Urbana, 1966.

[342] S. Wolfram, Cellular Automata and Complexity, Addison-Wesley, Reading, MA, 1994.

[343] N.T.J. Bailey, The Mathematical Theory of Infectious Diseases and Its Applications, Hafner Press, New York, 1975.

[344] R.M. Anderson, R.M. May, Infectious Diseases in Humans, Oxford University Press, Oxford, 1992.

[345] J.D. Murray, Mathematical Biology, Springer, Berlin, 1993.

[346] O. Diekmann, J. Heesterbeek, Mathematical Epidemiology of Infectious Diseases: Model Building, Analysis and Interpretation, Wiley, New York, 2000.

[347] H.W. Hethcote, SIAM Rev. 42 (2000) 599.

[348] P. Grassberger, Math. Biosci. 63 (1983) 157.

[349] H.J. Herrmann, Phys. Rep. 3 (1986) 153.

[350] J. Marro, R. Dickman, Nonequilibrium Phase Transitions in Lattice Models, Cambridge University Press, Cambridge, 1999. 
[351] A.L. Lloyd, R.M. May, Science 292 (2001) 1316.

[352] R.M. May, A.L. Lloyd, Phys. Rev. E 64 (2001) 66112.

[353] R.M. May, R.M. Anderson, Phil. Trans. R. Soc. Lond. B 321 (1988) 565.

[354] L.M. Sander, C.P. Warren, I.M. Sokolov, C. Simon, J. Koopman, Math. Biosci. 180 (2002) 293.

[355] M.E.J. Newman, Phys. Rev. E 66 (2002) 016128.

[356] F. Liljeros, C.R. Edling, L.A.N. Amaral, H.E. Stanley, Y. Aberg, Nature 411 (2001) 907.

[357] A. Schneeberger, et al., Sexual. Transmitt. Dis. 31 (2004) 380.

[358] Y. Moreno, R. Pastor-Satorras, A. Vespignani, Eur. Phys. J. B 26 (2002) 521.

[359] R. Pastor-Satorras, A. Vespignani, Phys. Rev. E 65 (2002) 035108(R).

[360] D.-U. Hwang, S. Boccaletti, Y. Moreno, R. Lopez-Ruiz, Math. Biosci. Eng. 2 (2005) 317.

[361] R. Pastor-Satorras, A. Vespignani, Phys. Rev. E 65 (2002) 036104.

[362] Z. Dezso, A.-L. Barabási, Phys. Rev. E 65 (2002) 055103R.

[363] P. Piot, et al., Nature 410 (2001) 968.

[364] R. Cohen, S. Havlin, D. ben-Avraham, Phys. Rev. Lett. 91 (2003) 247901.

[365] P. Holme, Europhys. Lett. 68 (2004) 908.

[366] V.M. Eguíluz, K. Klemm, Phys. Rev. Lett. 89 (2002) 108701.

[367] D. Volchenkov, L. Volchenkova, P. Blanchard, Phys. Rev. E 66 (2002) 46137.

[368] Y. Moreno, J.B. Gómez, A.F. Pacheco, Phys. Rev. E 68 (2003) 35103.

[369] M. Boguñá, R. Pastor-Satorras, A. Vespignani, Phys. Rev. Lett. 90 (2003) 28701.

[370] I. Schwartz, L. Billings, E. Bollt, Phys. Rev. E 70 (2004) 046220.

[371] F.S. Vannucchi, S. Boccaletti, Math. Biosci. Eng. 1 (2004) 49.

[372] J. Verdasca, et al., J. Theoret. Biol. 233 (2005) 553.

[373] W. Vogels, R. van Renesse, K. Birman, The power of epidemics: robust communication for large-scale distributed systems, in: the Proceedings of HotNets-I, Princeton, NJ, 2002.

[374] A.-M. Kermarrec, A. Ganesh, L. Massoulie, IEEE Trans. Parall. Distr. Syst. 14 (2003) 248.

[375] A.J. Demers, D.H. Greene, C. Hauser, W. Irish, J. Larson, Epidemic Algorithms for Replicated Database Maintenance, in: Proceedings of the Sixth Annual ACM Symposium on Principles of Distributed Computing, Vancouver, Canada, 1987.

[376] D.H. Zanette, Phys. Rev. E 64 (2001) 050901(R).

[377] Z. Liu, Y.-C. Lai, N. Ye, Phys. Rev. E 67 (2003) 031911.

[378] D.J. Daley, J. Gani, Epidemic Modelling, Cambridge University Press, Cambridge, UK, 2000.

[379] I. Foster, C. Kesselman (Eds.), The Grid: Blueprint for a Future Computing Infrastructure, Morgan Kaufman, San Francisco, 1999.

[380] D.J. Daley, D.G. Kendall, Nature 204 (1964) 1118.

[381] Y. Moreno, M. Nekovee, A. Vespignani, Phys. Rev. E 69 (2004) 055101(R).

[382] Y. Moreno, M. Nekovee, A.F. Pacheco, Phys. Rev. E 69 (2004) 066130.

[383] D. Hansel, H. Sompolinsky, Phys. Rev. Lett. 68 (1992) 718.

[384] F. Pasemann, Physica D 128 (1999) 236.

[385] H.G. Winful, L. Rahman, Phys. Rev. Lett. 65 (1990) 1575.

[386] R. Li, T. Erneux, Opt. Commun. 99 (1993) 196.

[387] R. Li, T. Erneux, Phys. Rev. A 49 (1994) 1301.

[388] K. Otsuka, R. Kawai, S. Hwong, J. Ko, J. Chern, Phys. Rev. Lett. 84 (2000) 3049.

[389] S. Jankowski, A. Londei, C. Mazur, A. Lozowski, Int. J. Electron. 79 (1995) 823.

[390] G. Filatrella, B. Straughn, P. Barbara, J. Appl. Phys. 90 (2001) 5675.

[391] C. Hugenii, Horoloquium Oscilatorium, Apud F. Muguet, Parisiis, 1673.

[392] S. Boccaletti, J. Kurths, D.L. Valladares, G. Osipov, C.S. Zhou, Phys. Rep. 366 (2002) 1.

[393] H. Fujisaka, T. Yamada, Prog. Theoret. Phys. 69 (1983) 32.

[394] V.S. Afraimovich, N.N. Verichev, M.I. Rabinovich, Radiophys. Quantum Electron. 29 (1986) 795.

[395] L.M. Pecora, T.L. Carroll, Phys. Rev. Lett. 64 (1990) 821.

[396] M.G. Rosenblum, A.S. Pikovsky, J. Kurths, Phys. Rev. Lett. 76 (1996) 1804.

[397] E.R. Rosa, E. Ott, M.H. Hess, Phys. Rev. Lett. 80 (1998) 1642.

[398] M.G. Rosenblum, A.S. Pikovsky, J. Kurths, Phys. Rev. Lett. 78 (1997) 4193.

[399] N.F. Rulkov, M.M. Sushchik, L.S. Tsimring, H.D.I. Abarbanel, Phys. Rev. E 51 (1995) 980.

[400] L. Kocarev, U. Parlitz, Phys. Rev. Lett. 76 (1996) 1816.

[401] S. Boccaletti, D.L. Valladares, Phys. Rev. E 62 (2000) 7497.

[402] M.A. Zaks, E.-H. Park, M.G. Rosenblum, J. Kurths, Phys. Rev. Lett. 82 (1999) 4228.

[403] R. Femat, G. Solis-Perales, Phys. Lett. A 262 (1999) 50.

[404] D.H. Zanette, Phys. Rev. E 55 (1997) 5315.

[405] P. Parmananda, Phys. Rev. E 56 (1997) 1595.

[406] A. Amengual, E. Hernández-García, R. Montagne, M. San Miguel, Phys. Rev. Lett. 78 (1997) 4379.

[407] S. Boccaletti, J. Bragard, F.T. Arecchi, H.L. Mancini, Phys. Rev. Lett. 83 (1999) 536.

[408] H. Chaté, A. Pikovsky, O. Rudzick, Physica D 131 (1999) 17.

[409] S. Boccaletti, D.L. Valladares, J. Kurths, D. Maza, H. Mancini, Phys. Rev. E 61 (2000) 3712.

[410] C. Schafer, M.G. Rosenblum, J. Kurths, H.H. Abel, Nature 392 (1998) 239. 
[411] P. Tass, M.G. Rosenblum, M.G. Weule, J. Kurths, A. Pikovsky, J. Volkmann, A. Schnitzler, H.J. Freund, Phys. Rev. Lett. 81 (1998) 3291.

[412] G.D. Van Wiggeren, R. Roy, Science 279 (1998) 1198.

[413] A. Neiman, X. Pei, D. Russell, W. Wojtenek, L. Wilkens, F. Moss, H.A. Braun, M.T. Huber, K. Voigt, Phys. Rev. Lett. 82 (1999) 660.

[414] D. Maza, A. Vallone, H. Mancini, S. Boccaletti, Phys. Rev. Lett. 85 (2000) 5567.

[415] G.M. Hall, S. Bahar, D.J. Gauthier, Phys. Rev. Lett. 82 (1999) 2995.

[416] A.R. Yehia, D. Jeandupreux, F. Alonso, M.R. Guevara, Chaos 9 (1999) 916.

[417] J.-W. Shuai, D.M. Durand, Phys. Lett. A 264 (1999) 289.

[418] C.M. Ticos, E. Rosa Jr., W.B. Pardo, J.A. Walkenstein, M. Monti, Phys. Rev. Lett. 85 (2000) 2929.

[419] E. Allaria, F.T. Arecchi, A. Di Garbo, R. Meucci, Phys. Rev. Lett. 86 (2001) 791.

[420] B. Blasius, A. Huppert, L. Stone, Nature 399 (1999) 354.

[421] D.J. DeShazer, R. Breban, E. Ott, R. Roy, Phys. Rev. Lett. 87 (2001) 044101.

[422] E. Barreto, P. So, B.J. Gluckmann, S.J. Schiff, Phys. Rev. Lett. 84 (2000) 1689.

[423] E. Barreto, P. So, Phys. Rev. Lett. 85 (2000) 2490.

[424] S. Boccaletti, L.M. Pecora, A. Pelaez, Phys. Rev. E 63 (2001) 066219.

[425] T. Nishikawa, A.E. Motter, Y.-C. Lai, F.C. Hoppensteadt, Phys. Rev. Lett. 91 (2003) 014101.

[426] L.F. Lago-Fernández, R. Huerta, F. Corbacho, J.A. Sigüenza, Phys. Rev. Lett. 84 (2000) 2758.

[427] P.M. Gade, C.-K. Hu, Phys. Rev. E 62 (2000) 6409.

[428] J. Jost, M.P. Joy, Phys. Rev. E 65 (2002) 016201.

[429] H. Hong, M.Y. Choi, B.J. Kim, Phys. Rev. E 65 (2002) 026139.

[430] O. Kwon, H.-T. Moon, Phys. Lett. A 298 (2002) 319.

[431] L.M. Pecora, T.L. Carroll, Phys. Rev. Lett. 80 (1998) 2109.

[432] K.S. Fink, G. Johnson, T.L. Carroll, L.M. Pecora, Phys. Rev. E 61 (2000) 5080.

[433] M. Barahona, L.M. Pecora, Phys. Rev. Lett. 89 (2002) 054101.

[434] Y. Chen, G. Rangarajan, M. Ding, Phys. Rev. E 67 (2003) 026209.

[435] G. Hu, J. Yang, W. Liu, Phys. Rev. E 58 (1998) 4440.

[436] M. Zhan, G. Hu, J. Yang, Phys. Rev. E 62 (2000) 2963.

[437] V.N. Belykh, I.V. Belykh, M. Hasler, Physica D 195 (2004) 159.

[438] P. Ashwin, J. Buescu, I. Stewart, Phys. Lett. A 193 (1994) 126.

[439] D.J. Gauthier, J.C. Bienfang, Phys. Rev. Lett. 77 (1996) 1751.

[440] S.A. Gerschgorin, Izv. Akad. Nauk. SSSR, Ser. Mat. 7 (1931) 749.

[441] H.E. Bell, Amer. Math. (Monthly) 72 (1965) 292.

[442] K. Josic, Communication at the 2005 SIAM Conference on Dynamical Systems.

[443] I. Belykh, et al., Phys. Rev. Lett. 94 (2005) 188101.

[444] G. Buzsáki, C. Geisler, D.A. Henze, X.J. Wang, Trends Neurosci. 27 (2004) 186.

[445] O. Sporns, D.R. Chialvo, M. Kaiser, C.C. Hilgetag, Trends Cogr. Sci. 8 (2004) 418.

[446] J. Bragard, S. Boccaletti, H. Mancini, Phys. Rev. Lett. 91 (2003) 064103.

[447] J. Bragard, S. Boccaletti, C. Mendoza, H.G.E. Hentschel, H. Mancini, Phys. Rev. E 70 (2004) 036219.

[448] A.E. Motter, C.S. Zhou, J. Kurths, Europhys. Lett. 69 (2005) 334.

[449] A.E. Motter, C. Zhou, J. Kurths, Phys. Rev. E 71 (2005) 016116.

[450] M. Chavez, D.-U. Hwang, A. Amann, H.G.E. Hentschel, S. Boccaletti, Phys. Rev. Lett. 94 (2005) 218701.

[451] D.M. Cvetkovic, M. Doob, H. Sachs, Spectra of Graphs: Theory and Applications, Johann Ambrosius Barth Verlag, Heidelberg, 1995.

[452] S.N. Dorogovtsev, J.F.F. Mendes, Phys. Rev. E 62 (2000) 1842.

[453] J.J. Ramasco, S.N. Dorogovtsev, R. Pastor-Satorras, Phys. Rev. E 70 (2004) 036106.

[454] D.-U. Hwang, M. Chavez, A. Amann, S. Boccaletti, Phys. Rev. Lett. 94 (2005) 138701.

[455] I.V. Belykh, V.N. Belykh, M. Hasler, Physica D 195 (2004) 188.

[456] D.J. Stilwell, E.M. Bollt, D.G. Roberson, Sufficient Conditions for Fast Switching Synchronization in Time Varying Network Topologies arXiv:nlin.CD/0502055 (2005).

[457] M.K.S. Yeung, S.H. Strogatz, Phys. Rev. Lett. 82 (1999) 648.

[458] M. Timme, F. Wolf, T. Geisel, Phys. Rev. Lett. 89 (2002) 258701.

[459] Y. Kuramoto, Chemical Oscillations, Waves, and Turbulence, Springer, Berlin, 1984.

[460] S.H. Strogatz, Physica D 143 (2000) 1.

[461] J.A. Acebrón, L.L. Bonilla, C.J. Pérez Vicente, F. Ritort, R. Spigler, Rev. Mod. Phys. 77 (2005) 137.

[462] Y. Moreno, A.F. Pacheco, Europhys. Lett. 68 (2004) 603.

[463] Y. Moreno, M. Vázquez-Prada, A.F. Pacheco, Physica A 343 (2004) 279.

[464] D.-S. Lee, Phys. Rev. E 72 (2005) 026208.

[465] T. Ichinomiya, Phys. Rev. E 70 (2004) 026116.

[466] J.G. Restrepo, E. Ott, B.R. Hunt, Phys. Rev. E 71 (2005) 036151.

[467] E. Oh, K. Rho, H. Hong, B. Kahng, Phys. Rev. E 72 (2005) 047101.

[468] P.N. MacGraw, M. Menzinger, Phys. Rev. E 72 (2005) 015101.

[469] C.W. Wu, L.O. Chua, IEEE Trans. Circ. Systems-I 42 (1995) 430.

[470] C.W. Wu, L.O. Chua, IEEE Trans. Circ. Systems-I 42 (1995) 494.

[471] C.W. Wu, L.O. Chua, IEEE Trans. Circ. Systems-I 43 (1996) 161. 
[472] J.F. Heagy, L.M. Pecora, T.L. Carroll, Phys. Rev. Lett. 74 (1995) 4185.

[473] L.M. Pecora, Phys. Rev. E 58 (1998) 347.

[474] J.G. Restrepo, E. Ott, B.R. Hunt, Phys. Rev. E 69 (2004) 066215.

[475] X.F. Wang, G. Chen, Int. J. Bifurcat. Chaos 12 (2002) 187.

[476] X. Li, G. Chen, IEEE Trans. Circ. Systems I 50 (2003) 1381.

[477] C.W. Wu, IEEE Trans. Circ. Systems I 50 (2003) 294.

[478] J. Lü, X. Yu, G. Chen, D. Chen, IEEE Trans. Circ. Systems I 51 (2004) 787.

[479] J. Lü, X. Yu, G. Chen, Physica A 334 (2004) 281.

[480] W. Lu, T. Chen, Physica D 198 (2004) 148.

[481] H. Hong, B.J. Kim, M.Y. Choi, H. Park, Phys. Rev. E 69 (2004) 067105.

[482] Y. Jiang, M. Lozada-Cassou, A. Vinet, Phys. Rev. E 68 (2003) 065201.

[483] C. Li, G. Chen, Physica A 341 (2004) 73.

[484] X.F. Wang, G. Chen, IEEE Trans. Circ. Systems I 49 (2002) 54.

[485] S. Jalan, R.E. Amritkar, Phys. Rev. Lett. 90 (2003) 014101.

[486] F.M. Atay, J. Jost, A. Wende, Phys. Rev. Lett. 92 (2004) 144101.

[487] C. Masoller, A.C. Martí, Phys. Rev. Lett. 94 (2005) 134102.

[488] L. Glass, Nature 410 (2001) 277.

[489] A. Winfree, J. Theor. Biol. 16 (1967) 15.

[490] A.S. Pikovsky, M.G. Rosenblum, J. Kurths, Europhys. Lett. 34 (1996) 165.

[491] I.Z. Kiss, Y. Zhai, J.L. Hudson, Science 296 (2002) 1676.

[492] S.H. Strogatz, R.E. Mirollo, Physica D 31 (1988) 143.

[493] P.C. Matthews, R.E. Mirollo, S.H. Strogatz, Physica D 52 (1991) 293.

[494] H. Sakaguchi, S. Shinomoto, Y. Kuramoto, Prog. Theoret. Phys. 77 (1987) 1005.

[495] R.R. Klevecz, J. Bolen, O. Durán, Int. J. Bifurcat. Chaos 2 (1992) 941.

[496] G.V. Osipov, A.S. Pikovsky, M.G. Rosemblum, J. Kurths, Phys. Rev. E 55 (1997) 2353.

[497] Z. Zheng, G. Hu, B. Hu, Phys. Rev. Lett. 81 (1998) 5318.

[498] V.N. Belykh, I.V. Belykh, M. Hassler, Phys. Rev. E 62 (2000) 6332.

[499] Y. Zhang, G. Hu, H.A. Cerdeira, S. Chen, T. Braun, Y. Yao, Phys. Rev. E 63 (2001) 026211.

[500] Z. Liu, Y.-C. Lai, F.C. Hoppensteadt, Phys. Rev. E 63 (2001) 055201.

[501] C.S. Zhou, J. Kurths, Phys. Rev. E 65 (2002) 040101.

[502] Y. Kuramoto, Prog. Theoret. Phys. 94 (1995) 321.

[503] Y. Kuramoto, H. Nakao, Phys. Rev. Lett. 76 (1996) 4352.

[504] Y. Kuramoto, H. Nakao, Physica D 103 (1997) 294.

[505] F. Rogister, K.S. Thornburg Jr., L. Fabiny, M. Möller, R. Roy, Phys. Rev. Lett. 92 (2004) 093905.

[506] J.L. Rogers, L.T. Wille, Phys. Rev. E 54 (1996) R2193.

[507] S.E.deS. Pinto, S.R. Lopes, R.L. Viana, Physica A 303 (2002) 339.

[508] M. Maródi, F. d'Ovidio, T. Vicsek, Phys. Rev. E 66 (2002) 011109.

[509] M.S.O. Massunaga, M. Bahiana, Physica D 168 (2002) 136.

[510] D. Sherrington, S. Kirkpatrick, Phys. Rev. Lett. 35 (1975) 1792.

[511] E. Niebur, H.G. Schuster, D.M. Kammen, C. Koch, Phys. Rev. E 44 (1991) 6895.

[512] S. Raghavachari, J.A. Glazier, Phys. Rev. Lett. 74 (1995) 3297.

[513] L.C. Freeman, Connections 19 (1996) 39.

[514] B. Wellman, J. Educ. Res. 14 (1926) 126.

[515] P.W. Holland, S. Leinhardt, Amer. J. Sociol. 76 (1970) 492.

[516] A. Bavelas, Human Organization 7 (1948) 16.

[517] P. Bonacich, Amer. J. Sociol. 92 (1987) 1170.

[518] G. Sabidussi, Psychometrika 31 (1965) 581.

[519] J. Nieminen, Scandinavian J. Psychol. 15 (1974) 322.

[520] K. Stephenson, M. Zelen, Social Networks 11 (1989) 1.

[521] R.N. Shepard, Science 210 (1980) 390.

[522] P.D. Hoff, A.E. Raftery, M.S. Handcock, J. Am. Statist. Assoc. 97 (2002) 1090.

[523] P. Killworth, H. Bernard, Social Networks 1 (1978) 159.

[524] T.H. Valente, R.K. Foreman, Social Networks 20 (1998) 89.

[525] D.L. Haynie, J. Quant. Criminol. 18 (2002) 99.

[526] W.E. Baker, R.B. Faulkner, Amer. Sociol. Rev. 58 (1993) 837.

[527] V. Krebs, Connections 24 (2002) 43.

[528] V. Latora, M. Marchiori, Chaos Sol. Fract. 20 (2004) 77.

[529] L.G. Frank, Animal Behav. 34 (1986) 1510.

[530] D. Lusseau, Proc. R. Soc. London B (Suppl.) 270 (2003) S186.

[531] D. Lusseau, M.E.J. Newman, Proc. R. Soc. London B (Suppl.) 271 (2004) S477.

[532] P. Gleiser, L. Danon, Adv. Complex Systems 6 (2003) 565.

[533] A. Arenas, L. Danon, A. Diaz-Aguilera, P.M. Gleiser, R. Guimerà, Eur. Phys. J. B 38 (2004) 373. 
[534] R.N. Onody, P.A. de Castro, Phys. Rev. E 70 (2004) 037103.

[535] D. de Lima e Silva, M. Medeiros Soares, M.V.C. Henriques, M.T. Schivani Alves, S.G. de Aguilar, T.P. de Carvalho, G. Corso, L.S. Lucena, Physica A 332 (2004) 559.

[536] J. Stiller, D. Nettle, R.I.M. Dunbar, Human Nature 14 (2003) 397.

[537] B. Wellman, J. Salaff, D. Dimitrova, L. Garton, M. Gulia, C. Haythornthwaite, Annu. Rev. Sociol. 22 (1996) 213.

[538] B. Wellman, Science 293 (2001) 2031.

[539] M.E.J. Newman, S. Forrest, J. Balthrop, Phys. Rev. E 66 (2002) 035101.

[540] K. Zhongbao, Z. Changshui, Phys. Rev. E 67 (2003) 036117.

[541] D. Garlaschelli, M.I. Loffredo, Phys. Rev. Lett. 93 (2004) 268701.

[542] W. Aiello, F. Chung, L. Lu, Random evolution of massive graphs, in: J. Abello, P.M. Pardalos, M.G.C. Resende (Eds.), Handbook of Massive Data Sets, Kluwer Academic, Dordrecht, 2002, p. 97.

[543] J. Wang, P. De Wilde, Phys. Rev. E 70 (2004) 066121.

[544] G. Caldarelli, F. Coccetti, P. De Los Rios, Phys. Rev. E 70 (2004) 027102.

[545] J.G. Ennis, Amer. Sociol. Rev. 57 (1992) 259.

[546] A.J. Lotka, J. Washington Acad. Sci. 16 (1926) 317.

[547] W. Shockley, Proc. IRE 45 (1957) 279.

[548] S. Redner, Eur. J. Phys. B 4 (1998) 131.

[549] C. Tsallis, M.P. de Albuquerque, Eur. J. Phys. B 13 (2000) 777.

[550] F. Menczer, Eur. Phys. J. B 38 (2004) 211.

[551] J.E. Hirsch, physics/0508025 (2005).

[552] P. Ball, Nature 436 (2005) 900.

[553] P.S. Bearman, K. Stovel, Poetics 27 (2000) 69.

[554] R. Ferrer i Cancho, R.V. Solé, Proc. R. Soc. London B 268 (2001) 2261.

[555] S.N. Dorogovtsev, J.F.F. Mendes, Proc. R. Soc. London B 268 (2001) 2603.

[556] A.E. Motter, A.P.S. de Moura, Y.C. Lai, P. Dasgupta, Phys. Rev. E 65 (2002) 065102.

[557] M. Sigman, G.A. Cecchi, Proc. Natl. Acad. Sci. USA 99 (2002) 1742.

[558] O. Kinouchi, A.S. Martinez, G.F. Lima, G.M. Lourenço, S. Risau-Gusman, Physica A 315 (2002) 665.

[559] R. Ferrer, I. Cancho, R.V. Solé, R. Köhler, Phys. Rev. E 69 (2004) 051915.

[560] M. Medeiros Soares, G. Corso, L.S. Lucena, Physica A 355 (2005) 678.

[561] A. Rapoport, Bull. Math. Biophys. 15 (1953) 523.

[562] J.F. Padgett, C.K. Ansell, Am. J. Sociol. 98 (1993) 1259.

[563] K. Faust, K.E. Willert, D.D. Rowlee, J. Skvoretz, Social Networks 24 (2002) 231.

[564] M.A. Porter, P.J. Mucha, M.E.J. Newman, C.M. Warbrand, Proc. Natl. Acad. Sci. USA 102 (2005) 7057.

[565] P.S. Dodds, D.J. Watts, C.F. Sabel, Proc. Natl. Acad. Sci. USA 100 (2003) 12516.

[566] S.P. Borgatti, P.C. Foster, J. Manage. 29 (2003) 991.

[567] S. Battiston, M. Catanzaro, Eur. Phys. J. B 38 (2004) 345.

[568] W.W. Powell, D.R. White, K.W. Koput, J. Owen-Smith, Amer. J. Sociol. 110 (2005) 1132.

[569] J.J. Potterat, R.B. Rothenberg, S.Q. Muth, Int. J. STD AIDS 10 (1999) 182.

[570] M.R. Weeks, S. Clair, S.P. Borgatti, K. Radda, J.J. Schensul, AIDS Behav. 6 (2002) 193.

[571] Y. Yaoum, E. Laumann, Sex. Trans. Dis. 29 (2002) 689.

[572] J.J. Potterat, S.Q. Muth, R.B. Rothenberg, H. Zimmerman-Roger, D.L. Green, J.E. Taylor, M.S. Bonney, H.A. White, Sex. Trans. Infect. 78 (Suppl. 1) (2002) i159.

[573] F. Liljeros, C.R. Edling, L.A.N. Amaral, Microbes and Infection 5 (2003) 189.

[574] K.T.D. Eames, M.J. Keeling, Math. Biosci. 189 (2004) 115.

[575] R.M. Anderson, R.M. May, Nature 333 (1989) 514.

[576] S.M. Blower, M.C. Samuel, J.A. Wiley, J. AIDS 5 (1992) 633.

[577] H. Jones, M.S. Handcock, Nature 423 (2003) 605.

[578] H. Jones, M.S. Handcock, Proc. R. Soc. Lond. B 270 (2003) 1123.

[579] M.S. Handcock, J.H. Jones, Theoret. Popul. Biol. 65 (2004) 413.

[580] E. Majorana, Scientia 36 (1942) 58.

[581] K. Sznajd-Weron, J. Sznajd, Int. J. Mod. Phys. C 11 (2000) 1157.

[582] G. Deffuant, D. Neau, F. Amblard, G. Weisbuch, Adv. Complex Systems 3 (2000) 87; G. Weisbuch, Eur. Phys. J. B 38 (2004) 339

[583] R. Hegselmann, U. Krause, J. Arti. Soc. Social Simul. 5 (3) (2002) (paper 2 jasss.soc.surrey.ac.uk).

[584] P.L. Krapivsky, S. Redner, Phys. Rev. Lett. 90 (2003) 238701; L.R. Fontes, R.H. Schonmann, V. Sidoravicius: Comm. Math. Phys. 228 (2002) 495

[585] C. Castellano, D. Vilone, A. Vespignani, Europhys. Lett. 63 (2003) 153.

[586] F. Wu, B.A. Huberman, Social Structure and Opinion Dynamics, cond-mat/0407252.

[587] K. Suchecki, V.M. Eguíluz, M. San Miguel, Europhys. Lett. 69 (2005) 228.

[588] R. Axelrod, J. Conflict Resolut. 41 (1997) 203.

[589] D. Stauffer, AIP Conf. Proc. 690 (2003) 147.

[590] D. Stauffer, physics/0503115. 
[591] S. Fortunato, D. Stauffer, cond-mat/0501730.

[592] E. Ben-Naim, P.L. Krapivsky, S. Redner, Physica D 183 (2003) 190.

[593] A. Pluchino, V. Latora, A. Rapisarda, cond-mat/0410217.

[594] S. Fortunato, preprint cond-mat/0408648.

[595] T. Yu-Song, A.O. Sousa, K. Ling-Jiang, L. Mu-Ren, preprint cond-mat/0501629, 2005.

[596] J. Bonnekoh, Int. J. Mod. Phys. 14 (2003) 1231.

[597] A.T. Bernardes, U.M.S. Costa, A.D. Araujo, D. Stauffer, Int. J. Mod. Phys. C 12 (2001) 159;

A.T. Bernardes, D. Stauffer, J. Kertész, Eur. Phys. J. B 25 (2002) 123

[598] M.C. Gonzales, A.O. Sousa, H.J. Herrmann, Int. J. Mod. Phys. C 15 (2004) 45.

[599] D. Stauffer, H. Meyer-Ortmanns, Int. J. Mod. Phys. C 15 (2004) 241.

[600] A.O. Sousa, cond-mat/0406766.

[601] S. Fortunato, Physica A 348 (2005) 683.

[602] M. He, H. Xu, Q. Sun, Int. J. Mod. Phys. C 15 (2004) 947.

[603] J. von Neumann, O. Morgenstern, Theory of Games and Economic Behavior, second ed., Princeton University Press, Princeton, 1947.

[604] M.J. Osborne, An Introduction to Game Theory, Oxford University Press, Oxford, 2002.

[605] R.J. Aumann, S. Hart (Eds.), Handbook of Game Theory with Economic Applications, North-Holland, Amsterdam, 1992.

[606] R. Swedberg, Theory and Society 30 (2001) 301.

[607] M.A. Nowak, K. Sigmund, Science 303 (2004) 793.

[608] A. Rapoport, A.M. Chammah, Prisoner's Dilemma, University of Michigan Press, Ann Arbor, 1965.

[609] M.A. Nowak, R.M. May, Nature 359 (1992) 826.

[610] R. Axelrod, The Evolution of Cooperation, Basic Books, New York, 1984.

[611] G. Abramson, M. Kuperman, Phys. Rev. E 63 (2001) 030901(R).

[612] B.J. Kim, A. Trusina, P. Holme, P. Minnhagen, J.S. Chung, M.Y. Choi, Phys. Rev. E 66 (2002) 021907.

[613] H. Ebel, S. Bornholdt, Phys. Rev. E 66 (2002) 056118.

[614] P. Holme, A. Trusina, B.J. Kim, P. Minnhagen, Phys. Rev. E 68 (2003) 030901.

[615] H. Ebel, S. Bornholdt, cond-mat/0211666.

[616] E. Lieberman, C. Hauert, M.A. Nowak, Nature 433 (2005) 312.

[617] M. Faloutsos, P. Faloutsos, C. Faloutsos, Comput. Commun. Rev. 29 (1999) 251.

[618] A. Vázquez, R. Pastor-Satorras, A. Vespignani, Phys. Rev. E 65 (2002) 66130.

[619] K.A. Eriksen, I. Simonsen, S. Maslov, K. Sneppen, Phys. Rev. Lett. 90 (2003) 148701.

[620] S. Maslov, K. Sneppen, A. Zaliznyak, arXiv:cond-mat 0205379 (2002).

[621] J. Park, M.E.J. Newman, Phys. Rev. E 68 (2003) 26112.

[622] C.R. Cunha, A. Bestavros, M.E. Crovella, Tech. Rep. (1995).

[623] R. Albert, H. Jeong, A.-L. Barabási, Nature 401 (1999) 130.

[624] B. Kahng, Y. Park, H. Jeong, Phys. Rev. E 66 (2002) 46107.

[625] A. Capocci, G. Caldarelli, P.D.L. Rios, Phys. Rev. E 68 (2003) 47101.

[626] D.M. Pennock, G.W. Flake, S. Lawrence, E.J. Glover, C.L. Giles, Proc. Natl. Acad. Sci. USA 99 (2002) 5207.

[627] R. Kumar, P. Raghavan, S. Rajalopagan, A. Tomkins, Proceedings of the 9th ACM Symposium on Principles of Database Systems, vol. 1, 1999.

[628] L.A. Adamic, Proceedings of the Third European Conference, ECDL'99, 1999, p. 443.

[629] L.A. Adamic, B.A. Huberman, Science 287 (2000) 2115.

[630] J.-P. Eckmann, E. Moses, Proc. Natl. Acad. Sci. USA 99 (2002) 5825.

[631] I. Nakamura, Phys. Rev. E 68 (2003) 45104.

[632] F. Menczer, Proc. Natl. Acad. Sci. USA 99 (2002) 14014.

[633] M. Argollo de Menezes, A.-L. Barabási, Phys. Rev. Lett. 93 (2004) 68701.

[634] L.H. Harwell, J.J. Hopfield, S. Leibler, A.W. Murray, Nature 402 (1999) C47.

[635] D.B. Searls, Nature Rev. 4 (2005) 45.

[636] B. Vogelstein, D. Lane, A.J. Levine, Nature 408 (2000) 307.

[637] N.A. Campbell, J.B. Reece, Biology, seventh ed., Pearson, 2001.

[638] B. Alberts, D. Bray, J. Lewis, M. Raff, K. Roberts, J.D. Watson, Molecular Biology of the Cell, third ed., Garland, 1994.

[639] R. Overbeek, N. Larsen, G.D. Pusch, M. DSouza, E. Selkov Jr., N. Kyrpides, M. Fonstein, N. Maltsev, E. Selkov, Nucl. Acids Res. 28 (2000) 123.

[640] P.D. Karp, M. Riley, S.M. Paley, A. Pellegrini-Toole, M. Krummenacker, Nucl. Acids Res. 30 (2002) 56.

[641] H. Ma, A.-P. Zeng, Bioinformatics 19 (2003) 270.

[642] H.-W. Ma, A.-P. Zeng, Bioinformatics 19 (2003) 1423.

[643] M. Arita, Proc. Natl. Acad. Sci. USA 101 (2004) 1543.

[644] R. Tanaka, Phys. Rev. Lett. 94 (2005) 168101.

[645] J. Podani, Z.N. Oltvai, H. Jeong, B. Tombor, A.-L. Barabási, E. Szathmáry, Nature Gen. 29 (2001) 54.

[646] P. Holme, M. Huss, H. Jeong, Bioinformatics 19 (2003) 532.

[647] S.-H. Yook, Z.N. Oltvai, A.-L. Barabási, Proteomics 4 (2004) 928.

[648] O.G. Vukmirovic, S.M. Tilghman, Nature 405 (2000) 820.

[649] D.J. Lockhart, E.A. Winzeler, Nature 405 (2000) 827. 
[650] D. Eisenberg, E.M. Marcotte, L. Xenarios, T.O. Yeates, Nature 405 (2000) 823.

[651] A. Pandey, M. Mann, Nature 405 (2000) 837.

[652] P. Uetz, L. Glot, G. Cagney, T.A. Mansfield, E. Al, Nature 403 (2000) 623.

[653] T. Ito, T. Chiba, R. Ozawa, M. Yoshida, M. Hattori, Y. Sakaki, Proc. Natl. Acad. Sci. USA 98 (2001) 4569.

[654] H.W. Mewes, D. Frishman, U. Güldener, G. Mannhaupt, K. Mayer, M. Mokrejs, B. Morgenstern, M. Münsterkötter, S. Rudd, B. Weil, Nucl. Acids Res. 30 (2002) 31.

[655] I. Xenarios, D.W. Rice, L. Salwinski, M.K. Baron, E.M. Marcotte, D. Eisenberg, Nucl. Acids Res. 28 (2000) 289.

[656] A. Wagner, Mol. Biol. Evol. 18 (2001) 1283.

[657] L.E.A. Giot, Science 302 (2003) 1727.

[658] S. Wuchty, Mol. Biol. Evol. 18 (2001) 1694.

[659] J. Park, M. Lappe, S.A. Teichmann, J. Mol. Biol. 307 (2001) 929.

[660] G. Apic, J. Gough, S.A. Teichmann, Bioinformatics 17 (2001) s83.

[661] R. Mahadevan, B.O. Palsson, Biophys. J.: Biophys. Lett. (2005) L07.

[662] A. Rzhetsky, S.M. Gomez, Bioinformatics 17 (2001) 988.

[663] A. Bhan, D.J. Galas, T.G. Dewey, Bioinformatics 18 (2002) 1486.

[664] A. Wagner, Proc. R. Soc. Lond. B 270 (2003) 457.

[665] R. Pastor-Satorras, E. Smith, R.V. Solé, J. Theoret. Biol. 222 (2003) 199.

[666] R. Mrowka, A. Patzak, H. Herzel, D. Holste, Phys. Rev. E 70 (2004) 51908.

[667] S.A. Romano, M.C. Eguia, Phys. Rev. E 71 (2005) 31901.

[668] E. Eisenberg, E.Y. Levanon, Phys. Rev. Lett. 91 (2003) 138701.

[669] U. Alon, Science 301 (2003) 1866.

[670] M.E. Wall, W.S. Hiavacek, M.A. Savageau, Nature Rev. Gen. 5 (2004) 34.

[671] S. Schuster, T. Pfeiffer, F. Moldenhauer, I. Koch, T. Dandekar, Bioinformatics 18 (2002) 351.

[672] B. Snel, P. Bork, M.A. Huynen, Proc. Natl. Acad. Sci. USA 99 (2002) 5890.

[673] M. Girvan, M.E.J. Newman, Proc. Natl. Acad. Sci. USA 99 (2002) 7821.

[674] A.W. Rives, T. Galitski, Proc. Natl. Acad. Sci. USA 100 (2003) 1128.

[675] V. Spirin, L.A. Mirny, Proc. Natl. Acad. Sci. USA 100 (2003) 12123.

[676] L. da, F. Costa, Phys. Rev. Lett. 93 (2005) 098702.

[677] J. Ihmels, G. Friedlander, S. Bergmann, Y.Z. Ofer Sarig, N. Barkai, Nature Gen. 31 (2002) 370.

[678] G.D. Bader, C.W.V. Hogue, Nature Biotechnol. 20 (2002) 991.

[679] S. Tornow, H.W. Mewes, Nucl. Acids Res. 31 (2003) 6283.

[680] R. Jansen, H. Yu, D. Greenbaum, Y. Kluger, N.J. Krogan, S. Chung, A. Emili, M. Snyder, J.F. Greenblatt, M. Gerstein, Science 302 (2003) 449.

[681] Z. Bar-Joseph, G.K. Gerber, T.I. Lee, N.J. Rinaldi, J.Y. Yoo, F. Robert, D.B. Gordon, E. Fraenkel, T.S. Jaakkola, R.A. Young, Nature Biotechnol. 21 (2003) 1337.

[682] R. Dobrin, Q.K. Beg, A.-L. Barabási, Z.N. Oltvai, BMC Bioinformatics 5 (2004) 10.

[683] S. Itzkovitz, R. Milo, N. Kashtan, G. Ziv, U. Alon, Phys. Rev. E 68 (2003) 26127.

[684] M. Csete, J. Doyle, Trends Biotechnol. 22 (2004) 446.

[685] F. Jacob, J. Monod, J. Mol. Bio. 3 (1961) 318.

[686] J. Gómez-Gardeñes, Y. Moreno, L.M. Floría, Physica A 352 (2005) 265;

J. Gómez-Gardeñes, Y. Moreno, L.M. Floría, Biophys. Chem. 115 (2005) 225

[687] S.A. Kauffman, The Origins of Order, Oxford University Press, New York, 1993.

[688] C. Oosawa, M.A. Savageau, Physica D 170 (2002) 143.

[689] R.V. Solé, I. Salazar-Ciudad, J. Garcia-Fernández, Physica A 305 (2002) 640.

[690] A. Goldbeter, D. Gonze, G. Houart, J.-C. Leloup, G. Dupont, Chaos 11 (2001) 247.

[691] D. Gonze, J. Hally, A. Goldbeter, Proc. Natl. Acad. Sci. USA 99 (2002) 673.

[692] O.A. Igoshin, A. Goldbeter, D. Kaiser, G. Oster, Proc. Natl. Acad. Sci. USA 101 (2004) 15760.

[693] N.S. Holter, M. Mitra, A. Maritan, M. Cieplak, J.R. Banavar, N.V. Fedroff, Proc. Natl. Acad. Sci. USA 97 (2000) 8409.

[694] O. Alter, P.O. Brown, D. Botstein, Proc. Natl. Acad. Sci. USA 97 (2000) 10101.

[695] J.J. Tyson, A. Csikasz-Nage, B. Novak, Bioessays 24 (2002) 1095.

[696] F. Varela, J.P. Lachaux, E. Rodriguez, J. Martinerie, Nat. Rev. Neurosci. 2 (2001) 229.

[697] C. Sotelo, Nat. Rev. Neurosci. 4 (2003) 71.

[698] R.R. Llinás, Nat. Rev. Neurosci. 4 (2003) 77.

[699] C. Koch, G. Laurent, Science 284 (1999) 96.

[700] A. Schüz, Neuroanatomy in a computational perspective, in: M.A. Arbib (Ed.), Handbook of Brain Theory and Neural Networks, MIT Press, Cambridge, 1995, p. 622.

[701] C. Cherniak, Trends Neurosci. 18 (1995) 522.

[702] R. Kötter, F. Sommer, Phil. Trans. R. Soc. London B Biol. Sci. 355 (2000) 127.

[703] V.A. Klyachko, C.F. Stevens, Proc. Natl. Acad. Sci. USA 100 (2003) 7937.

[704] C. Cherniak, Z. Mokhtarzada, R. Rodriguez-Esteban, K. Changizi, Proc. Natl. Acad. Sci. USA 101 (2004) 1081.

[705] D.B. Chklovskii, A.A. Koulakov, Ann. Rev. B Neurosci. 27 (2004) 369.

[706] A. Schnitzler, J. Gross, Nat. Rev. Neurosci. 6 (2005) 285. 
[707] A. Destexhe, E. Marder, Nature 431 (2004) 789.

[708] S.L. Bressler, Brain Res. Rev. 20 (1995) 288.

[709] A.K. Engel, P. Fries, W. Singer, Nat. Rev. Neurosci. 2 (2001) 704.

[710] G. Tononi, G.M. Edelman, Science 282 (1998) 1846.

[711] M. Hämäläinen, R. Hari, R. Ilmoniemi, J. Knuutila, O.V. Lounasmaa, Rev. Mod. Phys. 65 (1993) 413.

[712] N.K. Logothetis, J. Pauls, M. Augath, T. Trinath, A. Oeltermann, Nature 412 (2001) 150.

[713] A.K. Engel, C.K.E. Moll, I. Fried, G.A. Ojemann, Nat. Rev. Neurosci. 6 (2005) 35.

[714] L. Lee, L.M. Harrison, A. Mechelli, Network: Comput. Neural Systems 14 (2003) R1.

[715] B. Horwitz, Neuroimage 19 (2003) 466.

[716] A.A. Fingelkurts, S. Kähkönen, Neurosci. Behav. Rev. 28 (2005) 827.

[717] J.W. Scannell, S. Grant, B.R. Payne, R. Baddeley, Phil. Trans. R. Soc. Lond. B Biol. Sci. 355 (2000) 21.

[718] K.J. Friston, C.D. Frith, P.F. Liddle, R.S.J. Frackowiak, J. Cereb. Blood Flow Metab. 13 (1993) 5.

[719] A.M. Aertsen, G.L. Gerstein, M.K. Habib, G. Palm, J. Neurophysiol. 61 (1989) 900.

[720] C. Buchel, J.T. Coull, K.J. Friston, Science 283 (1999) 1538.

[721] J. Gross, J. Kujala, M. Hämäläinen, L. Timmermann, A. Schnitzler, R. Salmelin, Proc. Natl. Acad. Sci. USA 98 (2001) 694.

[722] K.E. Stephan, L. Kamper, A. Bozkurt, G.A.P.C. Burns, M.P. Young, R. Kötter, Phil. Trans. R. Soc. Lond. B Biol. Sci. 356 (2001) 1159.

[723] M. Bota, H.-W. Dong, L.W. Swanson, Nat. Neurosci. 6 (2003) 795.

[724] G.A.P.C. Burns, M.P. Young, Phil. Trans. R. Soc. Lond. B Biol. Sci. 355 (2000) 55.

[725] D. Felleman, D. van Essen, Cerebral Cortex 1 (1991) 1.

[726] M.P. Young, Proc. R. Soc. Lond. B Biol. Sci. 252 (1993) 13.

[727] J.W. Scannell, M.P. Youg, Curr. Biol. 3 (1993) 191.

[728] J.W. Scannell, C. Blackmore, M.P. Young, J. Neurosci. 15 (1995) 1463.

[729] J.W. Scannell, G.A.P.C. Burns, C.C. Hilgetag, M.A. O'Neill, M.P. Young, Cerebral Cortex 9 (1999) 279.

[730] J.G. White, E. Southgate, J.N. Thomson, S. Brenner, Phil. Trans. R. Soc. Lond. B Biol. Sci. 314 (1986) 1.

[731] M.B. Goodman, D.H. Hall, L. Avery, S.R. Lockery, Neuron 20 (1998) 763.

[732] C.C. Hilgetag, M.P. Young, M.A. O’Neill, Science 271 (1996) 776.

[733] M.P. Young, Nature 358 (1992) 152.

[734] M.P. Young, J.W. Scannell, M.A. O’Neill, C.C. Hilgetag, G. Burns, C. Blackmore, Phil. Trans. R. Soc. Lond. B Biol. Sci. 348 (1995) 281.

[735] K.E. Stephan, C.C. Hilgetag, G.A.P.C. Burns, M.A. O’Neill, M.P. Young, R. Kötter, Phil. Trans. R. Soc. Lond. B Biol. Sci. 355 (2000) 111.

[736] G. Ress, G. Kreiman, C. Koch, Nat. Rev. Neurosci. 3 (2002) 261.

[737] C.C. Hilgetag, G.A.P.C. Burns, M.A. O’Neill, J.W. Scannell, M.P. Young, Phil. Trans. R. Soc. Lond. B Biol. Sci. 355 (2000) 91.

[738] A.J. Goodhill, M.W. Simmen, D.J. Willshaw, Phil. Trans. R. Soc. Lond. B Biol. Sci. 348 (1995) 265.

[739] J. Vezoli, A. Falchier, B. Jouve, K. Knoblauch, M. Young, H. Kennedy, The Neuroscientist 10 (2004) 476.

[740] H. de Fraysseix, P. Kuntz, Algorithms Rev. 2 (1992) 105.

[741] B. Jouve, P. Rosentiehl, M. Imbert, Cerebral Cortex 8 (1998) 28.

[742] O. Sporns, G. Tononi, G.M. Edelman, Cerebral Cortex 10 (2000) 127.

[743] O. Sporns, J.D. Zwi, Neuroinformatics 2 (2004) 145.

[744] C.C. Hilgetag, M. Kaiser, Neuroinformatics 2 (2004) 353.

[745] O. Shefi, I. Golding, R. Segev, E. Ben-Jacob, A. Ayali, Phys. Rev. E 66 (2002) 021905.

[746] C. Cherniak, M. Changizi, D.W. Kang, Phys. Rev. E 59 (1999) 6001.

[747] J. Karbowski, Phys. Rev. Lett. 86 (2001) 3674.

[748] D. B Chklovskii, T. Schikorski, C.F. Stevens, Neuron 34 (2002) 341.

[749] R. Martin, M. Kaiser, P. Andras, M.P. Young, Is the brain a scale-free network?, Abstr. 31st Soc. Neurosci. Annual Meeting.

[750] R.E. Passingham, K.E. Sthepan, R. Kötter, Nat. Rev. Neurosci. 3 (2002) 606.

[751] F. Crick, C. Koch, Nature 391 (1998) 245.

[752] C.C. Hilgetag, A. Grant, Phil. Trans. R. Soc. Lond. B Biol. Sci. 355 (2000) 7.

[753] R. Kötter, C.C. Hilgetag, K.E. Stephan, Neurocomputing 38 (2001) 741.

[754] R. Kötter, K.E. Stephan, Neural Networks 16 (2003) 1261.

[755] O. Sporns, R. Kötter, PLOs Biol. 2 (2004) 1910.

[756] M.P. Young, C.C. Hilgetag, J.W. Scannell, Phil. Trans. R. Soc. Lond. B Biol. Sci. 355 (2000) 147.

[757] M. Kaiser, C.C. Hilgetag, Biol. Cybernet. 90 (2004) 311.

[758] C.J. Stam, Neurosci. Lett. 355 (2004) 25.

[759] S. Dodel, J.M. Herrmann, T. Geisel, Neurocomputing 44 (2002) 1065.

[760] V.M. Eguíluz, D.R. Chialvo, G.A. Cecchi, M. Baliki, A.V. Apkarian, Phys. Rev. Lett. 94 (2005) 018102.

[761] M.A. Koch, D.G. Norris, M. Hund-Georgiadis, Neuroimage 16 (2002) 241.

[762] D. Cordes, V.M. Haughton, K. Arfanakis, J.D. Carew, P.A. Turski, C.H. Moritz, M.A. Quigley, M.E. Meyerand, Am. J. Neuroradiol. 22 (2001) 1326.

[763] C. Holmgren, T. Harkany, B. Svennenfors, Y. Zilberter, J. Physiol. 551 (2003) 2003.

[764] S. Song, P.J. Sjöstrom, M. Reigl, S. Nelson, D.B. Chklovskii, PLOS Biol. 3 (2005) e68.

[765] M. Kaiser, C.C. Hilgetag, Neurocomputing 58 (2004) 297.

[766] M. Tsodyks, T. Kenet, A. Grinvald, A. Arieli, Science 286 (1999) 1943.

[767] G. Tononi, O. Sporns, G.M. Edelman, Proc. Natl. Acad. Sci. USA 91 (1994) 5033. 
[768] G. Tononi, O. Sporns, G.M. Edelman, Proc. Natl. Acad. Sci. USA 96 (1999) 3257.

[769] M. De Lucia, M. Bottaccio, M. Montuori, L. Pietronero, Phys. Rev. E 71 (2005) 016114.

[770] G. Tononi, A.R. McIntosh, D.P. Russell, G.M. Edelman, Neuroimage 7 (1998) 133.

[771] M.J.A.M. van Putten, C.J. Stam, Phys. Lett. A 281 (2001) 131.

[772] D. Golomb, J. Rinzel, Physica D 72 (1994) 259.

[773] C. van Vreeswijk, H. Sompolinsky, Science 274 (1996) 1724.

[774] D. Wang, IEEE Trans. Neural Networks 6 (1995) 941.

[775] D. Terman, D. Wang, Physica D 81 (1995) 148.

[776] X.J. Wang, G. Buzsáki, J. Neurosci. 16 (1996) 6402.

[777] D. Golomb, D. Hansel, Neural Comput. 12 (2000) 1095.

[778] H. Sompolinsky, A. Crisanti, Sommers, Phys. Rev. Lett. 61 (1988) 259.

[779] A.A. Minai, W.B. Levy, Biol. Cybernet. 70 (1993) 177.

[780] C.F. Mari, Phys. Rev. Lett. 85 (2000) 210.

[781] C. Mehring, U. Hehl, M. Kubo, M. Diesmann, A. Aertsen, Biol. Cybern. 88 (2003) 395.

[782] A.L. Hodgkin, A.F. Huxley, J. Physiol. (London) 117 (1952) 500.

[783] R. FitzHugh, Biophys. J. 1 (1961) 445.

[784] J. Nagumo, S. Arimoto, S. Yoshizawa, Proc. IRE 50 (1962) 2061.

[785] L. Lago-Fernandez, F.J. Corbacho, R. Huerta, Neural Networks 14 (2001) 687.

[786] L. Gammaitoni, P. Hänggi, P. Jung, F. Marchesoni, Rev. Mod. Phys. 70 (1998) 223.

[787] A.S. Pikovsky, J. Kurths, Phys. Rev. Lett. 78 (1997) 775.

[788] Z. Gao, B. Hu, G. Hu, Phys. Rev. E 65 (2001) 016209.

[789] S.H. Li, H.L. Wand, Q. Ouyang, Chin. Phys. 13 (2004) 448.

[790] L. Fortuna, M. Frasca, M. La Rosa, A. Spata, Chaos 15 (2005) 014102.

[791] M. Bucolo, L. Fortuna, M. La Rosa, Chaos Sol. Fract. 14 (2002) 1059.

[792] N. Masuda, K. Aihara, Biol. Cybernet 90 (2003) 302.

[793] S. Cosenza, P. Crucitti, L. Fortuna, M. Frasca, M. La Rosa, C. Stagni, L. Usai, Math. Biosci. Eng. 2 (2005) 53.

[794] X. Guardiola, A. Di`az-Guilera, M. Llas, C.J. Pérez, Phys. Rev. E 62 (2000) 5565.

[795] C.M. Gray, P. König, A.K. Engel, W. Singer, Nature 338 (1989) 334.

[796] A. Roxin, H. Riecke, S.A. Solla, Phys. Rev. Lett. 92 (2004) 198101.

[797] D. Simard, L. Nadeau, H. Kröger, Phys. Lett. A 336 (2005) 8.

[798] J.J. Hopfield, Proc. Natl. Acad. Sci. USA 79 (1982) 2445.

[799] D. O'Kane, A. Treves, J. Phys. A: Math. Gen. 25 (1992) 5055.

[800] S.S. Venkatesh, IEEE Trans. Inf. Theory 38 (1992) 1114.

[801] J. Komlós, R. Paturi, J. Comput. System Sci. 47 (1993) 350.

[802] N.L.D. Horn, E. Ruppin, Neural Comput. 11 (1999) 1717.

[803] J.W. Bohland, A.A. Minai, Neurocomputing 38 (2001) 489.

[804] P.N. McGraw, M. Menzinger, Phys. Rev. E 68 (2003) 047102.

[805] L.G. Morelli, G. Abramson, M.N. Kuperman, Eur. Phys. J. B 38 (2004) 495.

[806] J.J. Torres, M.A. Muñoz, J. Marro, P.L. Garrido, Neurocomputing 58 (2004) 229.

[807] B.J. Kim, Phys. Rev. E 69 (2004) 045101R.

[808] D. Stauffer, A. Aharony, L.daF. Costa, J. Adler, Eur. Phys. J. B 32 (2003) 395.

[809] L.daF. Costa, D. Stauffer, Physica A 330 (2003) 37.

[810] T.I. Netoff, R. Clewley, S. Arno, T. Keck, J.A. White, J. Neurosci. 24 (2004) 8075.

[811] G. Buzsáki, J.J. Chrobak, Curr. Opin. Neurobiol. 5 (1995) 504.

[812] M.A. Whittington, R.D. Traub, N. Kopell, B. Ermentrout, E.H. Buhl, Int. J. Psycophysiol. 38 (2000) 315.

[813] C. Börgers, N. Kopell, Neural Comput. 15 (2003) 509.

[814] M. Le Van Quyen, P. Quilichini, C. Bernard, M. Esclapez, Y. Ben-Ari, H. Gozlan, Interneuron-dependent fast oscillations at seizure onset in the immature brain, submitted for publication.

[815] L.C. Jia, M. Sano, P.Y. Lai, C.K. Chan, Phys. Rev. Lett. 93 (2004) 088101.

[816] L.daF. Costa, L. Diambra, Phys. Rev. E 71 (2005) 021901.

[817] C. Li, G. Chen, Phys. Rev. E 68 (2003) 052901.

[818] N. Kopell, B. Ermentrout, Proc. Natl. Acad. Sci. USA 101 (2004) 15482.

[819] D.B. Chklovskii, B.W. Mel, K. Svoboda, Nature 431 (2004) 782.

[820] S.R. Campbell, D.L. Wang, C. Jayaprakash, Neural Comput. 11 (1999) 1595.

[821] D.M. Wilkinson, B.A. Huberman, Proc. Natl. Acad. Sci. USA 101 (2004) 5241.

[822] A. Vespignani, Evolution thinks modular, Nature Gen. 35 (2003) 118.

[823] K.M. Hall, K.M. Hall, Manag. Sci. 17 (1970) 219.

[824] W.E. Donath, A.J. Hoffman, IBM J. Res. Dev. 17 (1973) 420.

[825] M. Fiedler, Czech. Math. J. 23 (1973) 298.

[826] A. Pothen, H. Simon, K.-P. Liou, SIAM J. Matrix Anal. Appl. 11 (1990) 430.

[827] B. Mohar, Discrete Math. 109 (1992) 171.

[828] A.J. Seary, W.D. Richards, Partitioning networks by eigenvectors, in: M.G. Everett, K. Rennolls (Eds.), Proceedings of the International Conference on Social Networks, vol. 1: methodology, 1996, pp. 47-58. 
[829] M.E.J. Newman, Eur. Phys. J. B 38 (2004) 321.

[830] M.R. Garey, D.S. Johnson, Computers and Intractability: A Guide to the Theory of NP-Completeness, W.H. Freeman \& Company, 1979.

[831] B. Kernighan, S. Lin. The Bell System Tech. J. 29 (1970) 291.

[832] C.M. Fiduccia, R.M. Mattheyses. Tech. Rep. 82CRD130, G. Corporate Research, 1982.

[833] B. Mohar, The Laplacian Spectrum of Graphs, Wiley, New York, 1991.

[834] R. Burioni, D. Cassi, A. Vezzani, Editor Vadim A. Kaimanovich, de Gruyter Berlin, 2004, p. 35.

[835] M. Fiedler, Czech. Math. J. 25 (1975) 619.

[836] G.H. Golub, C.F. Van Loan, Matrix Computations, Johns Hopkins University Press, Baltimore, 1996.

[837] W.W. Zachary, J. Anthropol. Res. 33 (1977) 452.

[838] V. Rosato, S. Bologna, F. Tiriticco, Topological properties of high-voltage electrical transmission networks, Electr. Power Systems Res., submitted for publication.

[839] G. Bonanno, F. Lillo, R.N. Mantegna, Quant. Finance 1 (2001) 96.

[840] S.C. Johnson, Psychometrika 2 (1967) 241.

[841] S.P. Borgatti, Connections 17 (1994) 78.

[842] F. Lorrain, H.C. White, J. Math. Sociol. 1 (1971) 49.

[843] R.S. Burt, Social Forces 55 (1976) 93.

[844] J.R. Tyler, D.M. Wilkinson, B.A. Huberman, in: M. Huysman, E. Wenger, V. Wulf (Eds.), Proceedings of the First International Conference on Communities and Technologies, Kluwer, Dordrecht, 2003.

[845] R. Guimerà, L. Danon, A. Díaz-Guilera, F. Giralt, A. Arenas, Phys. Rev. E 68 (2003) 065103.

[846] M. Boguñá, R. Pastor-Satorras, A. Díaz-Guilera, A. Arenas, Phys. Rev. E 70 (2004) 056122.

[847] D. Lusseau, M.E.J. Newman, preprint q-bio.PE/0403029.

[848] P. Holme, M. Huss, in: R. Gauges, U. Kummer, J. Pahle, U. Rost (Eds.), Proceedings of the 3rd Workshop on Computation of Biochemical Pathways and Genetic Networks, Logos, Berlin, 2003, pp. 3-9.

[849] F. Wu, B.A. Huberman, Eur. Phys. J. B 38 (2004) 331.

[850] M.E.J. Newman, Phys. Rev. E 70 (2004) 056131.

[851] S. Fortunato, V. Latora, M. Marchiori, Phys. Rev. E 70 (2004) 056104.

[852] H. Zhou, Phys. Rev. E 67 (2003) 041908.

[853] H. Zhou, Phys. Rev. E 67 (2003) 061901.

[854] http://www.mpikg-golm.mpg.de/th/people/zhou/NetworkCommunity.html

[855] M. Young, J. Sager, G.Csárdi, P. Hága, preprint cond-mat/0408263.

[856] M.E.J. Newman, Phys. Rev. E 69 (2004) 066133.

[857] A. Clauset, M.E.J. Newman, C. Moore, Phys. Rev. E 70 (2004) 066111.

[858] A. Capocci, V.D.P. Servedio, G. Caldarelli, F. Colaiori, Physica A 352 (2005) 669.

[859] L. Donetti, M.A. Muñoz, J. Stat. Mech. (2004) P10012.

[860] J. Reichardt, S. Bornholdt, Phys. Rev. Lett. 93 (2004) 218701.

[861] B.J. Kim, C.N. Yoon, S.K. Han, H. Jeong, Phys. Rev. E 65 (2002) 27103.

[862] H.J. Herrmann, D.C. Hong, H.E. Stanley, J. Phys. A: Math. Gen. 17 (1984) L261.

[863] J.D. Noh, H. Rieger, Phys. Rev. Lett. 92 (2004) 118701.

[864] L. Adamic, R.M. Lukose, A.R. Puniyani, B.A. Huberman, Phys. Rev. E 64 (2001) 46135.

[865] A.P.S. de Moura, A.E. Motter, C. Grebogi, Phys. Rev. E 68 (2003) 36106.

[866] H. Zhu, Z.-X. Huang, Phys. Rev. E 70 (2004) 36117.

[867] D.J. Watts, P.S. Dodds, M.E.J. Newman, Science 296 (2002) 1302.

[868] A. Trusina, M. Rosvall, K. Sneppen, Phys. Rev. Lett. 94 (2005) 238701.

[869] S.A. Levin, Ecosystems 1 (1998) 431.

[870] P.W. Anderson, K. Arrow, D. Pines, The Economy as an Evolving Complex System, Addison-Wesley, Redwood City, CA, 1988.

[871] M.E.J. Newman, D.J. Watts, S.H. Strogatz, Proc. Natl. Acad. Sci. USA 99 (2002) 2566.

[872] S. Jain, S. Krishna, Phys. Rev. Lett. 81 (1998) 5684.

[873] S. Bornholdt, T. Rohlf, Phys. Rev. Lett. 84 (2000) 6114.

[874] B. Derrida, J. Phys. A 20 (1987) L721.

[875] B. Derrida, E. Gardner, A. Zippelius, Europhys. Lett. 4 (1987) 167.

[876] R. Kree, A. Zippelius, Phys. Rev. A 36 (1987) 4421.

[877] A. Galstyan, K. Lerman, Phys. Rev. E 66 (2002) 015103(R).

[878] D. Challet, Y.-C. Zhang, Physica A 246 (1997) 207.

[879] M. Paczuski, K.E. Bassler, A. Corral, Phys. Rev. Lett. 84 (2000) 3185.

[880] D.Y.C. Chan, B.D. Hughes, A.S. Leong, W.J. Reed, Phys. Rev. E 68 (2003) 066124.

[881] J. Szymánski, in: M. Karonśki, A. Ruciński (Eds.), Random Graphs '87 (Poznam 1987), Wiley, New York, 1990 , pp. $313-324$.

[882] W.J. Reed, B.D. Hughes, Scientiae Mathematicae Japonicae Online 8 (2003) 329.

[883] M.G. Zimmermann, V.M. Eguíluz, M. San Miguel, Phys. Rev. E 69 (2004) 065102(R).

[884] S. Wang, C. Zhang, Phys. Rev. E 70 (2004) 066127.

[885] T. Vicsek, A. Czirók, E. Ben-Jacob, I. Cohen, O. Shochet, Phys. Rev. Lett. 75 (1995) 1226.

[886] G. Grégoire, H. Chaté, Phys. Rev. Lett. 92 (2004) 025702.

[887] A. Zaslaver, A.E. Mayo, R. Rosenberg, P. Bashkin, H. Sberro, M. Tsalyuk, M.G. Surette, Uri Alon, Nature Genetics 36 (2004) 486.

[888] U. Alon, Science 301 (2003) 1866. 